\title{
CARBON DIOXIDE REDUCTION ON GADOLINIA-DOPED CERIA CATHODES
}

\author{
by \\ ROBERT DAVID GREEN
}

Submitted in partial fulfillment of the requirements

for the degree of Doctor of Philosophy

Dissertation Adviser: Dr. Chung-Chiun Liu

Department of Chemical Engineering

CASE WESTERN RESERVE UNIVERSITY

May, 2009 


\section{CASE WESTERN RESERVE UNIVERSITY \\ SCHOOL OF GRADUATE STUDIES}

We hereby approve the thesis/dissertation of

Robert D. Green

candidate for the Doctor of Philosophy degree *.

(signed) $\frac{\text { Chung-Chiun Liu }}{\text { (chair of the committee) }}$
Stuart B. Adler

Uziel Landau

Mark R. DeGuire

J. Adin Mann

(date) November 7, 2008

*We also certify that written approval has been obtained for any proprietary material contained therein. 


\section{DEDICATION}

To Bill Wagar, a friend, and David Bixler, a relative, both of whom lost their battle with cancer as I began this endeavor,

and,

To my wife, Yook-Fung, who entered and blessed my life as I reached the end of this endeavor 


\section{Table of Contents}

Title $\quad$ i

Dedication $\quad$ iii

Table of Contents $\quad$ iv

List of Tables vi

List of Figures vii

Acknowledgements $\quad$ xi

Abbreviations Used xiii

Nomenclature Used xiv

Abstract xvii

Chapter 1. Introduction and Literature Review for Carbon Dioxide Reduction on Platinum and Solid Oxide Electrodes

1.1 Introduction

1.2 A Description of the Solid Oxide Electrolysis Process 4

1.3 Prior Research 5

$\begin{array}{lr}1.4 \text { Proposed Research } & 12\end{array}$

$\begin{array}{ll}\text { References } & 17\end{array}$

Chapter 2 Finite Element Analyses of Reference Electrode Placement on Thick Electrolytes to Quantify the Effect on EIS Measurements 21

$\begin{array}{ll}2.1 \text { Introduction } & 21\end{array}$

2.2 Theory and Computational Model Formulation $\quad 30$

2.3 Analysis and Computational Methods 41

2.4 Results and Discussion $\quad 48$

2.5 Conclusions $\quad 71$

$\begin{array}{ll}\text { References } & 74\end{array}$

Chapter 3 Development and Fabrication of Gadolinia-Doped Ceria Electrode $\begin{array}{ll}\text { Solid Oxide Electrolysis Cells } & 77\end{array}$

$\begin{array}{ll}3.1 \text { Introduction } & 77\end{array}$

3.2 Choice of $40 \mathrm{~mol} \%$ Gadolinia-Doped Ceria for Electrode 77

3.3 Fabrication of the Solid Oxide Symmetric Cells 92

3.4 Experimental Setup $\quad 97$

$\begin{array}{ll}3.5 \text { Summary } & 101\end{array}$

$\begin{array}{ll}\text { References } & 102\end{array}$

Chapter 4 The $\mathrm{CO}-\mathrm{CO}_{2}$ Exchange Porous Electrode Model 106

$\begin{array}{ll}4.1 \text { Introduction } & 106\end{array}$

$\begin{array}{ll}4.2 \text { Theory } & 111\end{array}$

$\begin{array}{ll}4.3 \text { Discussion } & 125\end{array}$

$\begin{array}{ll}4.4 \text { Summary } & 128\end{array}$ 
Chapter 5 EIS Measurements of $\mathrm{CO}-\mathrm{CO}_{2}$ Exchange on GDC Electrodes 132

5.1 Introduction 132

5.2 Analysis and Data Reduction 133

5.3 Results and Discussion 136

$\begin{array}{ll}5.4 \text { Conclusions } & 160\end{array}$

5.5 Future Work 161

5.6 Closing Remarks $\quad 162$

References 162

Appendix A Implementation of the Pellet Geometry Model in the Finite Element Analysis Package, COMSOL Multiphysics ${ }^{\circledR} \quad 167$

Appendix B Description and Calibration of Oxygen Sensor to Measure $\mathrm{CO} / \mathrm{CO}_{2}$ Gas Mixture Ratios 171

Appendix C Derivation of the Relationship Between the Surface Reaction Rate Constant, $k$, and Surface Exchange Coefficient, $k_{s}$

Appendix D Derivation of the GDC Oxygen Non-stoichiometry Model and the Resulting Relationship for the Thermodynamic Factor, $A \quad 179$

Appendix E Derivation of the CO- $\mathrm{CO}_{2}$ Exchange Model for a Non-Zero Vacancy Flux at the Current Collector Surface 186

Appendix F The Rate Expression for the CO- $\mathrm{CO}_{2}$ Exchange Reaction 192

Appendix G A Brief Introduction to Kröger-Vink Notation 196

Appendix H Sample MATLAB M-file listing for Pellet Geometry Analyses 198

Appendix I Sample MATLAB M-file listing for fitting impedance data to $\mathrm{CO}-\mathrm{CO}_{2}$ exchange model 206

$\begin{array}{ll}\text { Bibliography } & 216\end{array}$ 


\section{List of Tables}

\section{Chapter 2}

Table 2-1. Description of pellet geometries for the 2D parametric analysis cases

Table 2-2. Maximum error in $|Z|$ due to misaligned electrodes

\section{Chapter 3}

Table 3-1. Some representative electronic and ionic conductivities of ceria and Gd-doped ceria at $1000^{\circ} \mathrm{C}$ and $P_{O_{2}}=10^{-15}$ atm

Table 3-2. Example electronic and ionic mobilities for several compositions of ceria at $1000^{\circ} \mathrm{C}$ and $P_{O_{2}} \approx 10^{-12}$ atm

Table 3-3. Thermal expansion coefficient (TEC) data for pure ceria and selected Gd doping levels

\section{Chapter 4}

Table 4-1. Dimensionless variables for derivation of $\mathrm{CO}-\mathrm{CO}_{2}$ exchange model

\section{Appendix E}

Table E-1. Comparison of fitted parameters for the $\mathrm{CO}-\mathrm{CO}_{2}$ model solved for zero and non-zero vacancy flux boundary conditions at the current collector interface 


\section{List of Figures}

\section{Chapter 1}

Figure 1-1. A basic description of a solid oxide cell for carbon dioxide electrolysis.

Figure 1-2. A qualitative illustration of the proposed mechanism of carbon dioxide reduction

Figure 1-3. A qualitative illustration of electronic-only conducting and mixed-conducting porous electrode design

\section{Chapter 2}

Figure 2-1. Diagram of a Luggin capillary commonly used as a reference electrode in aqueous electrochemistry studies

Figure 2-2. Diagram of the "Risø pellet" cell

Figure 2-3. Diagram of the baseline thick pellet cell geometry

Figure 2-4. Diagram of the 2D axisymmetric pellet geometry defining the computational subdomain and associated boundary conditions

Figure 2-5. Electrical analog of cell model

Figure 2-6. Nyquist plots for samples of the impedance responses for the two boundary condition electrode models

Figure 2-7. A typical mesh for the pellet subdomain

Figure 2-8. A surface plot of a typical finite element analysis (FEA) solution for the pellet geometry

Figure 2-9. Computational results for the current density along the radius of the working electrode (WE)

Figure 2-10. Impedance spectral response simulation of symmetric electrodes for the baseline cell geometry

Figure 2-11. Impedance spectral response simulation of the baseline cell geometry with non-linear but symmetric WE and CE 
Figure 2-12. Nyquist plots of the baseline cell geometry with non-symmetric electrodes

Figure 2-13. Nyquist plot of the Case 1 cell geometry with non-symmetric electrodes

Figure 2-14. Maximum error in $|Z|$ results for the parametric analyses of the cylindrical pellet geometry

Figure 2-15. 3D model geometry of pellet cell for working electrode (WE) offset analysis

Figure 2-16. Variation in the edge reference electrode (RE2) potential over the circumference of the $1 / 2$ pellet $3 \mathrm{D}$ geometry

Figure 2-17. Bode plot of the $\Delta \theta$ error for the $0.17 \mathrm{~mm}$ offset case

Figure 2-18. Experimental comparison of RE1 and full cell measurements

\section{Chapter 3}

Figure 3-1. Oxygen non-stoichiometry data for pure ceria and gadolinia-doped ceria, $x=0.10,0.20$, and 0.30

Figure 3-2. An X-ray diffraction (XRD) scan of the $40 \mathrm{~mol} \% \mathrm{Gd}$-doped $\mathrm{CeO}_{2}$ powder used to fabricate the GDC cells in this study

Figure 3-3. Measured lattice parameter for $40 \mathrm{~mol} \% \mathrm{Gd}$-doped ceria along with lattice parameter data for pure ceria and at Gd-doping levels of $10-40 \mathrm{~mol} \%$

Figure 3-4. Scanning electron micrographs of the YSZ electrolyte surface of a die-cast pellet

Figure 3-5. Photographs of a fabricated GDE symmetric electrode pellet cell

Figure 3-6. Test fixture setup for AC impedance measurements in a single atmosphere test chamber

Figure 3-7. Schematic diagram of $\mathrm{CO} / \mathrm{CO}_{2}$ gas flow system

Figure 3-8. Photo of gas flow system installed in walk-in vented hood 


\section{Chapter 4}

Figure 4-1. Geometry and model domain for 1-dimensional $\mathrm{CO}-\mathrm{CO}_{2}$ exchange porous electrode model

Figure 4-2 Dimensionless vacancy concentration vs. electrode thickness for several values of the utilization thickness ratio, $\phi$

Figure 4-3. Examples of different responses of derived $\mathrm{CO}-\mathrm{CO}_{2}$ exchange model for different values of the utilization thickness ratio

\section{Chapter 5}

Figure 5-1. Scanning electron micrographs of porous $\mathrm{Gd}_{0.4} \mathrm{Ce}_{0.6} \mathrm{O}_{1.75}$ electrode fired onto a YSZ electrolyte pellet at $1250^{\circ} \mathrm{C}$

Figure 5-2. Nyquist plot of AC impedance spectrum of GDC/YSZ/GDC cell after 103 hours of operation

Figure 5-3. Arrhenius plot of area specific resistance (ASR) over the range of temperature and $\mathrm{CO} / \mathrm{CO}_{2}$ gas mixture ratios tested

Figure 5-4. AC impedance spectra responses with variation in $\mathrm{CO} / \mathrm{CO}_{2}$ gas mixture ratio

Figure 5-5. Fitted parameters from $\mathrm{CO}-\mathrm{CO}_{2}$ exchange model, equation (5-1), as a function of partial pressure of $\mathrm{O}_{2}$

Figure 5-6. Fitted parameters for the $\mathrm{CO}-\mathrm{CO}_{2}$ exchange model, equation (5-1), as a function of reciprocal temperature

Figure 5-7. $D_{v}$ data as a function of reciprocal temperature, extracted from measured impedance data on a $40 \mathrm{~mol} \%$ GDC electrode

Figure 5-8. $D_{v}$ data as a function of the partial pressure of $\mathrm{O}_{2}$, extracted from measured impedance data on a $40 \mathrm{~mol} \%$ GDC electrode

Figure 5-9. Exchange rate, $\mathfrak{R}_{0}$, extracted from impedance data for $\mathrm{CO} / \mathrm{CO}_{2}$ exchange on a $40 \mathrm{~mol} \%$ GDC electrode as a function of partial pressure of $\mathrm{O}_{2}$

Figure 5-10. Comparison of impedance spectra of a Pt/YSZ/Pt cell with a GDC/YSZ/GDC cell 
Figure 5-11. Change in impedance spectra with operational time

\section{Appendix B}

Figure B-1. Pt/YSZ/Pt oxygen sensor potential measurements made for calibration

\section{Appendix D}

Figure D-4. Ideal point theory defect model fit to $40 \mathrm{~mol} \%$ GDC data

\section{Appendix E}

Figure E-1. Comparison of CO- $\mathrm{CO}_{2}$ model fits for a zero flux and a non-zero vacancy flux boundary condition at the current collector interface 


\section{Acknowledgements}

First of all, as a non-traditional, "mature" graduate student, I truly feel blessed for the opportunity I had to return to graduate school and pursue a $\mathrm{PhD}$ in mid-career. Although not easy, I do consider my experience another example demonstrating it is never too late to continue your education and pursue an advanced degree.

I wish to thank Prof. Stuart B. Adler at the University of Washington. Prof. Adler was my original advisor at Case, and provided me with that crucial help that most graduate students need, namely, a focused and well-defined set of objectives of the research problem that I brought with me that was of interest to my employer, NASA. After leaving Case, he continued to advise me remotely and I am indebted to him for his continued advice, especially with the development of the $\mathrm{CO}-\mathrm{CO}_{2}$ exchange model, and careful review of my work. I wish to thank Prof. C.C. Liu who become my advisor at Case after Prof. Adler left, and patiently guided and advised me through the reminder of this effort, gently but firmly prodding me when my progress was slow!

I want to thank my dissertation committee of Profs. Uziel Landau, Jay Mann, and Mark DeGuire who graciously volunteered to serve on my dissertation committee and provided both candid and constructive criticism of my work. 
I also want to thank John Setlock, Thomas Cable, and Steven Sofie (Ceramics Branch, NASA GRC) for many helpful discussions on cell fabrication and ceramic processing methods. I wish to thank David Hull (Advanced Metallics Branch, NASA GRC) for the SEM and EDS measurements, and Rick Rodgers (also of the Advanced Metallics Branch, GRC) for XRD measurements and a number of consultations to determine and address the existence of a secondary phase in my electrode material.

I would like to acknowledge Bhim Singh, John McQuillen, and Brian Motil for encouraging me and allowing me a respite from my daily workload at NASA to complete this work. Also, John McQuillen provided detailed comments on my early work by carefully reviewing a Solid State Ionic journal article published prior to this work. I thank Lynn Mallinak, also at NASA, who helped me with scanning a number of my figures and binding copies of my dissertation for review. I am grateful to the NASA Glenn Research Center (GRC) Independent Research and Development (IR\&D) Fund and the NASA Exploration Systems Mission Directorate (ESMD), for providing the funding for this work.

Finally, pursuing a Ph.D. does require hard work, intense focus, and long hours. I thank my parents for instilling the hard work ethic into me at an early age. I thank my wife for all her understanding and support, and willingness to attend my defense to see exactly what I was doing all these years! And, I thank God for all the skill and abilities to perform research at this level, along with patience, wisdom, and perseverance in this journey. 
ASR

CE

$\mathrm{COE}$

CFM

EIS

ESB

GDC

ID

IEDP

ISRU

LSCF

MIEC

NASA

OD

ODE

PTFE

RE1

RE2

SCCM

SDC

SEM

SIMS

SOE

SOFC

TEC

WE

XRD

YSZ area specific resistance, in ohms-cm ${ }^{2}$

counter electrode

closed one end

cubic feet per minute

Electrochemical impedance spectroscopy

Erbium stabilized bismuth oxide, $\mathrm{Bi}_{x} \mathrm{Er}_{1-x} \mathrm{O}_{1.5-\delta}$

Gadolinia-Doped Ceria, $\mathrm{Ce}_{1-x} \mathrm{Gd}_{x} \mathrm{O}_{2-\delta}$

inside diameter

Isotope Exchange/Depth Profiling

In-Situ Resource Utilization

Lanthanum strontium cobalt iron oxide

mixed ionic-electronic conductor

National Aeronautics and Space Administration

outside diameter

ordinary differential equation

polytetrafluoroethylene (e.g. Teflon ${ }^{\mathrm{TM}}$ )

Reference Electrode 1

Reference Electrode 2

standard cubic centimeters per minute

Samaria Doped Ceria, $\mathrm{Ce}_{1-x} \mathrm{Sm}_{x} \mathrm{O}_{1.5}$

Scanning Electron Microscope

Secondary Ion Mass Spectroscopy

Solid Oxide Electrolysis

Solid Oxide Fuel Cell

Thermal expansion coefficient

working electrode

$\mathrm{X}$-ray diffraction

Yttria-Stabilized Zirconia 


\section{Nomenclature Used}

\begin{tabular}{|c|c|c|}
\hline$A$ & dimensionless & thermodynamic factor \\
\hline $\mathrm{A}_{e l}$ & $\mathrm{~cm}^{2}$ & electrode area (imprinted on electrolyte) \\
\hline $\mathrm{a}$ & angstroms & lattice parameter (for fluorite phase) \\
\hline$a$ & $\mathrm{~cm}^{-1}$ & surface area per unit volume \\
\hline$C_{W E}$ & Farads-cm ${ }^{2}$ & area-specific capacitance of the working electrode \\
\hline$D^{*}$ & $\frac{\mathrm{cm}}{\mathrm{sec}}$ & oxygen tracer diffusion coefficient \\
\hline$D_{v}$ & $\frac{\mathrm{cm}}{\mathrm{sec}}$ & vacancy diffusion coefficient \\
\hline$D_{v}^{e f f}$ & $\frac{\mathrm{cm}}{\mathrm{sec}}$ & effective vacancy diffusion coefficient $\left(\frac{D_{v}}{\tau_{s}}\right)$ \\
\hline$F$ & $96487 \frac{\mathrm{C}}{\mathrm{mol}}$ & Faraday's constant \\
\hline$F_{\min }$ & & $\begin{array}{l}\text { objective function to be minimized using non-linear } \\
\text { least squares analysis (in Chapter 5) }\end{array}$ \\
\hline$f$ & $\mathrm{~Hz}$ & frequency \\
\hline$f_{\mathrm{O}_{2}}$ & atm & fugacity of oxygen in the gas \\
\hline$f_{\mathrm{O}_{2}}^{\text {solid }}$ & atm & fugacity of oxygen in the solid \\
\hline$\Delta G^{0}$ & $\mathrm{~J} / \mathrm{mol}$ & $\begin{array}{l}\text { standard Gibbs free energy of reaction (for GDC } \\
\text { oxygen non-stoichiometry reaction) }\end{array}$ \\
\hline$H_{p}$ & $\mathrm{~cm}$ & Height of electrolyte pellet (for Chapter 2 analyses) \\
\hline$h_{p}$ & $\mathrm{~cm}$ & $\begin{array}{l}\text { height of reference electrode hole (for Chapter } 2 \\
\text { analyses) }\end{array}$ \\
\hline$i$ & $\frac{\mathrm{A}}{\mathrm{cm}^{2}}$ & current density \\
\hline$j$ & $\sqrt{-1}$ & imaginary unity \\
\hline$K_{e q}$ & dimensionless & chemical equilibrium constant \\
\hline$k$ & $\frac{\mathrm{mol}}{\mathrm{cm}^{2}-\mathrm{sec}}$ & surface reaction constant \\
\hline$k_{s}$ & $\frac{\mathrm{mol}}{\mathrm{cm}^{2}-\mathrm{sec}}$ & surface exchange constant \\
\hline$L$ & $\mathrm{~cm}$ & length (or thickness) of electrode \\
\hline$l_{\delta}$ & $\mathrm{cm}$ & characteristic length (or thickness) of electrode (see \\
\hline $\begin{array}{l}n \\
\mathbf{n}\end{array}$ & $\begin{array}{l}\text { dimensionless } \\
\text { dimensionless }\end{array}$ & $\begin{array}{l}\text { Table 4-1) } \\
\text { number of data points, integer value } \\
\text { unit normal vector for a surface }\end{array}$ \\
\hline
\end{tabular}




$\begin{array}{ll}P_{O_{2}} & \text { atm } \\ R & 8.314 \frac{\mathrm{J}}{\mathrm{mol}-\mathrm{K}} \\ R_{\text {chem }} & \text { ohms-cm } \\ R_{W E} & \text { ohms-cm } \\ \mathfrak{R}_{0} & \frac{\mathrm{mol}}{\mathrm{cm}^{2}-\mathrm{sec}} \\ r & \frac{\mathrm{mol}}{\mathrm{cm}^{2}-\mathrm{sec}} \\ t & \text { sec } \\ t_{\text {chem }} & \text { sec } \\ t_{e} & \text { dimensionless } \\ \mathbf{x} & \mathrm{cm} \\ x_{i} & \text { dimensionless } \\ y & \text { cm } \\ Z & \text { ohms-cm } \\ Z_{i}^{\text {data }} & \text { ohms-cm } \\ Z^{\text {fit }} & \\ Z_{i}^{\text {fit }} & \text { ohms-cm } \\ Z_{W E} & \text { ohms-cm } \\ Z_{\text {chem }} & \text { ohms-cm } \\ |Z| & \text { ohms-cm } \\ \mid Z & \end{array}$

partial pressure of oxygen gas

ideal gas constant

characteristic resistance of electrode

area-specific resistance of the working electrode

surface exchange rate coefficient

reaction rate

independent variable for time

characteristic time constant of electrode

electronic transference number

position vector

mole fraction of component $i$

1-D length variable for electrode

AC impedance

individual impedance data point (in Chapter 5)

complex-valued fitting function (in Chapter 5)

individual impedance value for fitting equation (in

Chapter 5)

Impedance of working electrode (in Chapter 2)

impedance due to chemical processes, i.e. diffusion, kinetics

magnitude of the impedance,

$=\sqrt{a^{2}+b^{2}}$ for $Z=a+b i$

Greek symbols

\begin{tabular}{|c|c|c|}
\hline$\Phi$ & $\mathrm{mV}$ & $\begin{array}{l}\text { complex quasi-static potential in electrolyte } \\
\text { computational domain }\end{array}$ \\
\hline$\tilde{\Phi}$ & $\mathrm{mV}$ & $\begin{array}{l}\text { position dependent complex potential in the } \\
\text { electrolyte computational domain }\end{array}$ \\
\hline$\hat{\Phi}$ & $\mathrm{mV}$ & $\begin{array}{l}\text { time dependent complex potential in electrolyte } \\
\text { computation domain }\end{array}$ \\
\hline & $\mathrm{mV}$ & complex electrode potential \\
\hline$\Psi$ & $\mathrm{mV}$ & position dependent complex electrode potential \\
\hline & $\mathrm{mV}$ & time dependent complex electrode potential \\
\hline & dimensionless & dimensionless current (see Table 4-1) \\
\hline & dimensionless & porosity of electrode \\
\hline
\end{tabular}




\begin{tabular}{|c|c|c|}
\hline$\phi$ & $\frac{L}{l_{\delta}}$ & dimensionless thickness of active electrode (see \\
\hline$\kappa$ & $\mathrm{S} \mathrm{cm}{ }^{2}$ & $\begin{array}{l}\text { Table } 4-1 \text { ) } \\
\text { electrolyte conductivity (in Chapter } 2 \text { ) }\end{array}$ \\
\hline$\lambda$ & & $\begin{array}{l}\text { vector of parameters for fitting function, } Z^{\text {fit }} \text { (in } \\
\text { Chapter 5) }\end{array}$ \\
\hline$\theta$ & radians & $\begin{array}{l}\text { phase angle of the impedance, } \\
=\operatorname{Arctan}\left(\frac{b}{a}\right) \text { for } Z=a+b i\end{array}$ \\
\hline$\theta$ & radians & also used for Bragg's angle (in Chapter 3) \\
\hline$\sigma$ & dimensionless & dimensionless frequency (see Table 4-1) \\
\hline$\tau_{s}$ & dimensionless & solid-phase tortuosity of electrode \\
\hline$\omega$ & $\frac{\text { radians }}{\sec }$ or $\sec ^{-1}$ & radial (or angular) frequency, $2 \pi f$ \\
\hline$\xi$ & dimensionless & dimensionless length (see Table 4-1) \\
\hline$\psi$ & dimensionless & dimensionless vacancy mole fract (see Table 4-1) \\
\hline
\end{tabular}

Superscripts

solid

$e q b$

refers to GDC solid or other solid oxide material at equilibrium

\section{Subscripts}

$C E$

el

$i$

$\mathrm{o}$

$\mathrm{O}_{2}$

$R E 1$

RE2

$v$

$W E$ counter electrode (in Chapter 2)

electrode

chemical species (or individual data point in

Chapter 5)

oxygen (referring to an oxygen lattice site)

oxygen (in gas form or in solid in equilibrium with

gas)

Reference Electrode 1 (referring to reference electrode position in the hole of the baseline cell geometry in Chapter 2)

Reference Electrode 2 (referring to reference

electrode position at the midpoint edge around the circumference of the baseline cell geometry in

Chapter 2)

vacancy in a crystal structure

working electrode (in Chapter 2) 
Carbon Dioxide Reduction on Gadolinia-Doped Ceria Cathodes

Abstract

By

ROBERT DAVID GREEN

This work describes an investigation of $\mathrm{CO}-\mathrm{CO}_{2}$ exchange on $40 \mathrm{~mol} \%$ gadoliniadoped ceria (GDC) electrodes for potential application as a $\mathrm{CO}_{2}$ reduction cathode for the solid oxide electrolysis of $\mathrm{CO}_{2}$.

A computational analysis was performed on the thick electrolyte cylindrical pellet test cell geometry to investigate the effects of this cell geometry on Electrochemical Impedance Spectroscopy (EIS) due to non-linear current distribution. The analysis showed the particular cell geometry selected does induce an error of $15 \%$ on the impedance measurements, but in a predicable linear manner. Additional parametric analyses indicate that impedance errors for the cylindrical cell geometry can be reduced by covering the faces of the pellet with the working and counter-electrodes, centering the reference electrode hole equidistant from working and counter-electrodes, or utilizing a reference electrode mounted at the midpoint edge of the pellet.

A continuum-based model is described for equilibrium $\mathrm{CO}-\mathrm{CO}_{2}$ exchange on a mixed-conducting electrode utilizing porous electrode theory. The resulting three- 
parameter model is expressed in terms of a characteristic resistance $\left(R_{\text {chem }}\right)$, a characteristic time constant $\left(t_{\text {chem }}\right)$, and a utilization thickness ratio $(\phi)$, that can be related to physiochemical properties.

EIS measurements were performed on the $40 \mathrm{~mol} \%$ GDC electrodes on yttria stabilized zirconia (YSZ) electrolytes at $700-950^{\circ} \mathrm{C}$ in reducing $\mathrm{CO} / \mathrm{CO}_{2}$ atmospheres. Area-specific-resistance (ASR) values for this electrode were in the range of 0.8-37 ohm$\mathrm{cm}^{2}$, about two orders of magnitude lower than measurements on Pt electrodes and slightly lower than data on Ni-YSZ electrodes in the literature under similar temperature and partial pressure of oxygen $\left(P_{\mathrm{O}_{2}}\right)$ conditions. An analysis utilizing the continuumbased porous electrode model was performed to extract the vacancy diffusion coefficient $\left(D_{v}\right)$ and surface exchange rate coefficient $\left(\Re_{0}\right)$ as a function of temperature and $P_{O_{2}}$, from the impedance results. The $D_{v}$ data agree well with published measurements of the tracer diffusion coefficient $\left(D^{*}\right)$ based on isotope profiling by secondary ion mass spectroscopy (SIMS), and conductivity measurements on $40 \mathrm{~mol} \%$ GDC. The $\mathfrak{R}_{0}$ values are a factor of 3 lower than published measurements of the surface reaction rate $(k)$ obtained from isothermal thermogravimetric relaxation and decrease with increasing $P_{\mathrm{O}_{2}}$. 


\section{CHAPTER 1}

\section{INTRODUCTION AND LITERATURE REVIEW FOR CARBON DIOXIDE REDUCTION ON PLATINUM AND SOLID OXIDE ELECTRODES}

\subsection{Introduction}

The National Aeronautics and Space Administration (NASA) is beginning to plan manned and unmanned exploratory missions to return to the Moon and eventually to Mars, as part of the Exploration Vision directed by the President in January 2004 [1]. As NASA begins to develop these more detailed plans to the Moon and Mars, it becomes clear the severe restrictions our present and near-term propulsion technologies impose on the mass and volume of unmanned or manned payloads that can be launched to our moon or a planet. For example, the 1960’s Apollo lunar module (or LM) design had a “4-to-1 weight growth factor”, i.e. for every pound increase that the LM ascent stage experienced during its engineering development, a corresponding cascade effect caused a 3 pound increase in the LM descent stage [2], resulting in a 4 pound weight gain to the total vehicle. Aerospace system trade studies have shown that generation of oxygen or other propellants from the available Martian natural resources, such as the atmosphere or regolith, would significantly reduce the launch up-mass requirements for chemical propulsion systems being considered for either unmanned Mars Sample Return (MSR) missions [3-5] or a manned mission to Mars [6-8]. The suite of potential chemical processes which could convert these extra-terrestrial resources into propellant or life 
support system products [9] (i.e. oxygen, water), are referred to as In-situ Resource Utilization (ISRU) technologies. They offer a promising means of enabling these types of missions that may not be technically feasible without the development of significantly larger payload capacity launch vehicles.

For a mission to Mars, the natural resource most readily available is the atmosphere, which consists of roughly 95\% carbon dioxide, a potential feedstock for the production of oxygen, methane, and other consumables. A key chemical step in any chemical process to manufacture these in-situ products is the reduction of this carbon dioxide, and a number of potential technologies have been proposed to address this challenge [10] and include photochemical reduction [11], plasma discharge, electrochemical-based carbon dioxide reduction approaches [12], and solid oxide electrolysis, the focus of this work.

Singh and Sridhar [13] discuss the advantages of carbon dioxide solid oxide electrolysis over some of these other potential competing technologies, namely the Sabatier/water electrolysis and reverse water gas shift reaction processes. They note the solid oxide electrolysis (SOE) process of reducing carbon dioxide to generate oxygen is a promising technology being considered for this application, one of its key advantages being the ability to produce a nearly pure dry oxygen gas stream without any additional chemical separation processes required. ${ }^{*}$ Another advantage of this device is that it can

\footnotetext{
* Production of pure dry oxygen for an aerospace application such as this is particularly
} advantageous, as any extra separation equipment adds weight and complexity to the spacecraft system. Dry 
be reversed and used as a fuel cell [14] (i.e. consuming carbon monoxide and oxygen) to generate power when solar power is not available, or for portable devices such as rovers.

The previous paragraphs describe the specific aerospace application(s) for carbon dioxide electrolysis. However, with the rise in global oil prices over the past two years, there appears to be a recent emerging interest in a related terrestrial application for this process, namely the feasibility of electrolyzing mixed carbon dioxide and water streams $[15,16]$. This co-electrolysis of $\mathrm{CO}_{2}$ and $\mathrm{H}_{2} \mathrm{O}$, in addition to the production of pure oxygen, produces a mixture of carbon monoxide (CO) and hydrogen $\left(\mathrm{H}_{2}\right)$ gas, or syngas, on the cathode side of the electrolyzer. The syngas product can be used as a raw material in the manufacture of synthetic fuels, lubrication oils, or other hydrocarbon products via the Fisher-Tropsch process [17]. A recent economic analysis [18] indicates that $\mathrm{CO}_{2}$ could be electrolyzed for a cost equivalent to a crude oil price of $\$ 40 /$ barrel, i.e. calculated by comparing the higher heating values (HHV) of oil and CO gas. If proven to be economically feasible, this process may also provide an alternative to carbon sequestration [19] for reducing global $\mathrm{CO}_{2}$ emissions from coal-fired electric power plants, or potentially, provide a market for $\mathrm{CO}_{2}$ removed directly from the atmosphere [20].

oxygen is of benefit if it is necessary to store the oxygen product as a cryogenic liquid, i.e. a small amount of water vapor in an oxygen gas stream may cause freezing problems in components of a cryogenic liquefaction system. 


\subsection{A Description of the Solid Oxide Electrolysis Process:}

Figure 1-1 illustrates the basic operation of the solid oxide electrolysis (SOE) process. The SOE electrolysis cell consists of an oxygen-ion conducting electrolyte (or membrane), typically yttria-stabilized zirconia (YSZ), coated on both sides with electrodes, typically a thin porous layer of platinum. A potential is applied across the electrodes and carbon dioxide is supplied to the cathode side where it dissociates into CO and $\mathrm{O}^{2-}$ ions:

$$
\mathrm{CO}_{2}+2 e^{-} \rightarrow \mathrm{CO}+\mathrm{O}^{2-}
$$

The carbon monoxide leaves in the carbon dioxide gas stream. The $\mathrm{O}^{2-}$ ions diffuse across the oxygen-ion conducting electrolyte and form pure oxygen on the anode side:

$$
O^{2-} \rightarrow \frac{1}{2} O_{2}+2 e^{-}
$$

As the YSZ electrolyte is a solid-state material and conducts only oxygen ions -- via oxygen vacancies -- in the crystal lattice, this process produces essentially pure oxygen requiring no additional separation processes. 


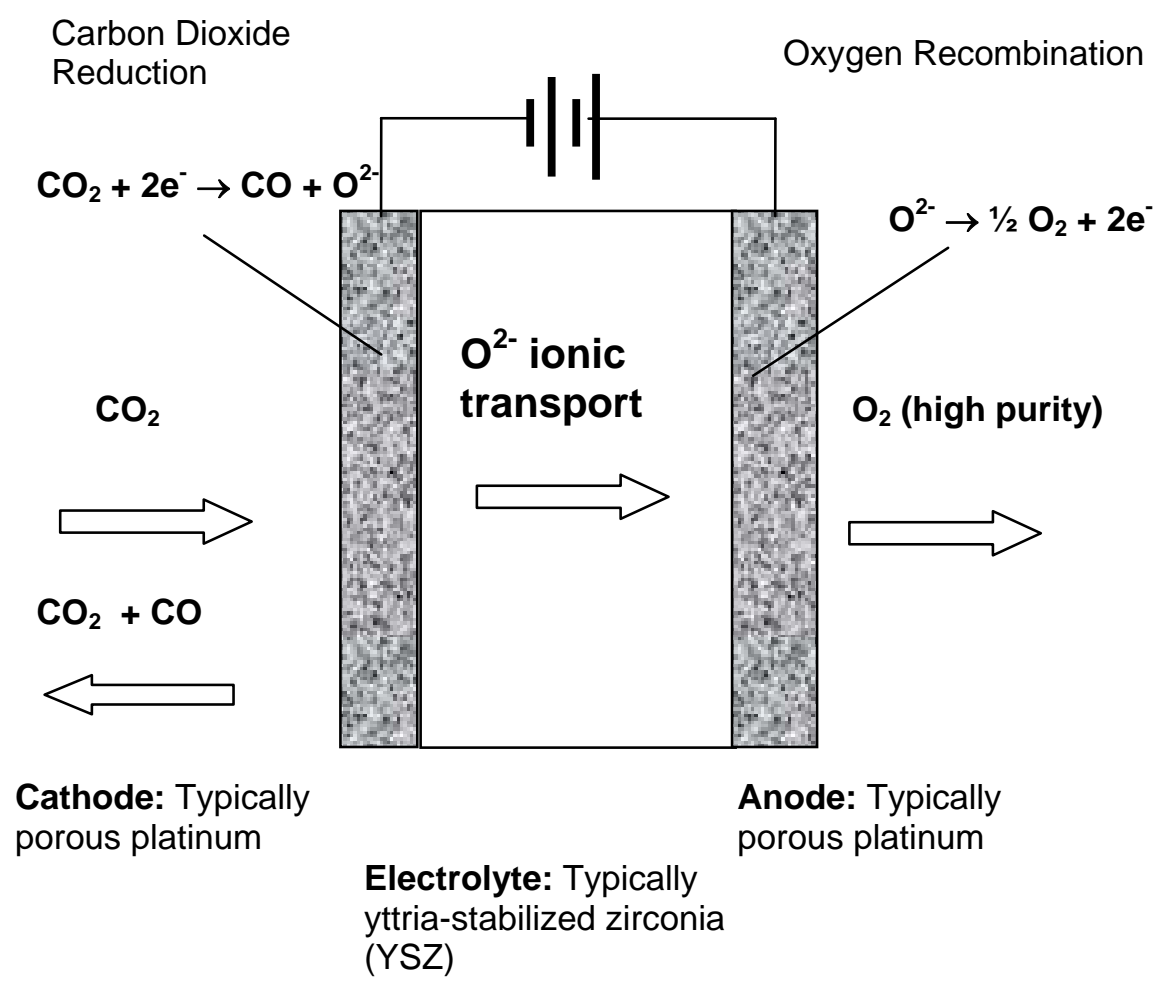

Figure 1-1. A basic description of a solid oxide cell for carbon dioxide electrolysis. Both the anode and cathode are porous electrodes, and are typically 10-50 microns thick, and the electrolyte can range from 10-300 microns in thickness, depending on whether electrode or electrolyte is providing structural support.

\subsection{Prior Research}

Although solid oxide electrolysis as an ISRU technology is a relatively new idea, the reduction of carbon dioxide for oxygen regeneration in spacecraft habitats was considered early in the US manned space-flight program. Elikan et al. [21] suggested the concept - which they referred to as a solid electrolyte "battery"- to electrolyze carbon dioxide and/or water to generate oxygen from the carbon dioxide and waste water 
products produced by an astronaut crew ${ }^{*}$; they presented thermodynamic calculations and preliminary engineering analyses to show the feasibility of this concept. Chandler and Pollara [22] built and tested a carbon dioxide reduction system consisting of a solid electrolyte cell and a “disproportionation” reactor (to further reduce the carbon monoxide product to carbon); their solid electrolyte cell was a Pt/YSZ/Pt type and they observed the oxygen production rate from their cells matched that predicted by Faraday's Law to within $1 \%$. Elikan et al. $[23,24]$ also built a breadboard system to reduce a mixture of carbon dioxide and water to recover oxygen for spacecraft life support applications; their electrolyzer consisted of a tubular YSZ cell stack with Pt-YSZ composite electrodes.

The first proposed use of solid oxide electrolysis (SOE) of carbon dioxide as an ISRU technology was suggested in the late 1970 's. Ash $^{\dagger}$ et al. [25] originally proposed this idea in 1978 as a potential method of generating oxygen from the predominately carbon dioxide atmosphere of Mars. Stancati et al. [26] mentions the process in a study

\footnotetext{
* Note that in order to utilize this technology, the carbon dioxide would have to be separated from
} the cabin air, as the solid oxide electrolyzer would preferentially reduce the more abundant oxygen in the cabin atmosphere. This separation can be done via adsorption compression using a zeolite adsorbent material. In fact, the presently on-orbit manned International Space Station utilizes such an adsorption compression system to remove $\mathrm{CO}_{2}$ from the cabin air; the adsorbed gas is then "dumped" overboard by exposing the zeolite to the vacuum of space.

${ }^{\dagger}$ Robert Ash has informally been given credit by the ISRU community for inventing the concept of ISRU, with the SOE process being the first proposed ISRU technology. Robert Zubrin is probably the more well-known and influential advocate, who developed the key arguments and rationale for ISRU as a critical enabling concept for a manned mission to Mars. 
of technologies that could be used to perform in-situ propellant production (ISPP) for an unmanned Mars sample return mission. Richter [27] performed some initial experimental work, measuring polarization curves under various operating conditions and determining the critical potential at which the solid electrolyte material (8 mol \% YSZ) is reduced and oxygen is extracted from the electrolyte. He also developed a simple thermodynamic model of the process to predict the contributions to the cell overpotential. Crow and Ramohalli $[28,29]$ initiated an extensive research program to develop an electrolysis stack using Pt electrodes and a single crystal YSZ electrolyte for each cell to avoid thermal cycling problems. They reported high oxygen conversion efficiencies but noted there was some evidence of air leaking through the edges of their cell, making their data suspect.

Other than these preliminary research and engineering demonstration efforts to develop a working SOE for carbon dioxide reduction, little fundamental research has been performed to gain a more detailed understanding of the carbon dioxide reduction reaction in a solid-state electrochemical system. This is probably due to the limited application to aerospace related life support and in-situ resource utilization (ISRU) research.

Conversely, a number of efforts have focused on the carbon monoxide oxidation reaction on platinum and various metal oxides in heterogeneous catalysis systems, related to development of catalysts for automotive emission systems. In fact, CO oxidation on Pt catalysts is probably among the most studied of all heterogeneous reactions [30]. A 
number of researchers have reported oscillatory variations in the carbon monoxide oxidation reaction on Pt and a number of other noble metal catalysts [30,31]. They attribute these oscillations to slow oxidation and reduction of the metal surface layer and variations in surface carbon concentration. Finally, Freund and Roberts [32] provide an excellent review of the surface chemistry of carbon dioxide on adsorbates, metals, transition metals and oxides.

Much of the electrochemical-based work on carbon monoxide oxidation has been done on Pt/YSZ/Pt cells, paralleling the early 1960's and 1970's solid oxide fuel cell research efforts. Karpachov et al. [33] made some of the earliest measurements of carbon monoxide oxidation on an electrochemical cell; they performed polarization measurements on a Ca-doped zirconia electrolyte cell with porous Pt electrodes, and observed a lower polarization with increased $\mathrm{CO} / \mathrm{CO}_{2}$ ratio which they attributed to accumulated atomic oxygen on the electrode surface due to the slow oxidation rate of CO on the anode. Etsell and Flengas [34] also made measurements on similar chemistry cells in low ratio $\mathrm{CO} / \mathrm{CO}_{2}$ atmospheres and reported Tafel parameter values of 0.5 for the transfer coefficients and exchange current densities on the order of $1 \mathrm{~mA} / \mathrm{cm}^{2}$; they concluded that charge transfer involving gaseous or adsorbed $\mathrm{CO}$, and gaseous $\mathrm{CO}_{2}$ was the rate limiting step. Mizusaki et al. [35] made steady state and impedance measurements also in low ratio $\mathrm{CO} / \mathrm{CO}_{2}$ mixtures, but concluded adsorbed $\mathrm{CO}$ on Pt and adsorbed oxygen ions on YSZ at the three phase boundary as rate limiting for the steadystate behavior, whereas they concluded chemical diffusion of adsorbed O on the YSZ surface was rate limiting for the transient (impedance) behavior. 
Metcalfe and Sundaresan [36] and Metcalfe [37] investigated carbon monoxide on Pt on YSZ and $\mathrm{Al}_{2} \mathrm{O}_{3}$ supports --related to enhancing automotive emission catalysts-- and were able to measure electrode exchange current densities of carbon monoxide oxidation using a solid electrolyte potentiometry technique with a three-electrode DC system. They observed enhanced carbon monoxide oxidation on $\mathrm{YSZ}$ vs. $\mathrm{Al}_{2} \mathrm{O}_{3}$ supports which they attribute to enhanced interfacial oxygen transfer due to the oxygen ion conductivity of YSZ vs. $\mathrm{Al}_{2} \mathrm{O}_{3}$. They also measured increased reaction rates for Pt dispersed on porous YSZ, $\mathrm{TiO}_{2}$ and ceria $\left(\mathrm{CeO}_{2}\right)$ supports, compared to $\mathrm{Al}_{2} \mathrm{O}_{3}$ porous supports. Sridhar and Foerstner [14] also performed some experimental work on carbon monoxide oxidation on Pt/YSZ in order to utilize their electrolyzer cell as a regenerative fuel cell, i.e. utilize the carbon monoxide and oxygen produced by SOE during the daylight hours as fuel for the fuel cell for nighttime electrical needs. They found the performance of the cell decreased during the first 40 hours of operation and became stable for greater operating times. They attribute this performance degradation to chemisorption of carbon monoxide on the platinum electrodes, and note that initial performance is recovered when the electrolysis cell is run in the electrolyzer mode for a few hours. Tao et al. [38] made polarization measurements on Pt/YSZ/Pt cells undergoing carbon dioxide electrolysis and reported the Butler-Volmer parameters for their data. In a companion paper [39], they describe results for carbon dioxide electrolysis on Pt-YSZ cermet (or composite, i.e. Pt-YSZ/YSZ/PtYSZ) electrode cells that they fabricated; they reported significantly increased performance, i.e. almost triple the current density, but attribute most of this improvement to the anode, as opposed to the cathode where $\mathrm{CO}_{2}$ reduction is occurring. 
Although the majority of carbon dioxide reduction work has been performed on $\mathrm{Pt}$ paste electrodes, some recent work has focused on alternative electrode materials. Lauvstad and Tunold [40] made polarization and impedance measurements on strontium ferrate $\left(\mathrm{SrFeO}_{3-\delta}\right)$ electrodes in $\mathrm{CO} / \mathrm{CO}_{2}$ mixture ratios of $0.153,1.54$, and $25.1 \mathrm{~mol} \% \mathrm{CO}$ in $\mathrm{CO}_{2}$; they observed increased charge transfer resistances at lower $\mathrm{CO} / \mathrm{CO}_{2}$ ratios, and propose a mechanism involving closed pores at the $\mathrm{YSZ} \mathrm{SrFeO}_{3-\delta}$ interface to explain their impedance results. They also observed a decrease in conductivity from the impedance measurements in the $25.1 \mathrm{~mol} \% \mathrm{CO}$ in $\mathrm{CO}_{2}$ mixture, which they attribute to possible decomposition of the electrode material in low $P_{\mathrm{O}_{2}}$ (i.e. $10^{-17}$ atm) atmospheres. Park and Wachsman [41] fabricated and tested cells consisting of a bilayer samaria doped ceria (SDC, $\mathrm{Ce}_{0.8} \mathrm{Sm}_{0.2} \mathrm{O}_{1.5}$ ) and erbium stabilized bismuth oxide ( $\mathrm{ESB}, \mathrm{Bi}_{0.8} \mathrm{Er}_{0.2} \mathrm{O}_{1.5}$ ) electrolyte with a Pt electrode for the $\mathrm{CO}_{2}$ reduction electrode and a composite Ag-ESB electrode for the anode. They measured polarization curves over lower temperatures $\left(500^{\circ}\right.$ to $\left.700^{\circ} \mathrm{C}\right)$ than for YSZ as an electrolyte and reported high Faradaic efficiencies for operation at $500^{\circ} \mathrm{C}$, but decreased efficiencies at $700^{\circ} \mathrm{C}$ due to electronic conduction through the bilayer electrolyte. Most recently, Bidrawn et al. [42] tested cells with a cathode consisting of $\mathrm{La}_{0.8} \mathrm{Sr}_{0.2} \mathrm{Cr}_{0.5} \mathrm{Mn}_{0.5} \mathrm{O}_{3}$ (LSCM), Pd, and ceria infiltrated in a porous YSZ layer and have reported $\mathrm{CO}_{2}$ electrolysis efficiencies similar to those for $\mathrm{H}_{2} \mathrm{O}$ electrolysis.

Most of the more recent work has focused on utilizing Ni on YSZ and/or Ni-YSZ cermets for carbon monoxide oxidation, following the extensive work on Ni-YSZ 
cermets as the state-of-the-art composite for solid oxide fuel cell anodes [43] utilizing hydrogen as a fuel. Aaberg et al. [44] performed both polarization and impedance measurements Ni/YSZ electrodes and reported polarization resistances 2-5 times higher for $\mathrm{CO}-\mathrm{CO}_{2}$ than for $\mathrm{H}_{2}-\mathrm{H}_{2} \mathrm{O}$; they reported that their area-specific-resistance (ASR) values reached a minimum at a $\mathrm{CO} / \mathrm{CO}_{2}$ ratio of 0.8 . Holtappels et al. [45] also performed polarization and AC impedance measurements on Ni-YSZ electrodes and observed periodic changes of the reaction rate (from 30 to 80 s), which they attribute to the passivation and reactivation of active sites of the $\mathrm{Ni} / \mathrm{YSZ}$ electrode surface. Matsuzaki and Yasuda [46] also made AC impedance measurements on Ni-YSZ cermet electrodes in $\mathrm{CO}-\mathrm{CO}_{2}$ and in various $\mathrm{H}_{2}-\mathrm{H}_{2} \mathrm{O}-\mathrm{CO}-\mathrm{CO}_{2}$ mixtures, observing the electrochemical oxidation rate for $\mathrm{CO}$ is $2-3$ times slower than for $\mathrm{H}_{2}$ under the same $P_{\mathrm{O}_{2}}$ conditions; they attribute this difference to the larger surface diffusion resistance of CO, compared to $\mathrm{H}_{2}$, at lower temperatures $\left(750^{\circ} \mathrm{C}\right)$ and a combination of diffusion and charge-transfer resistances at higher temperatures $\left(1000^{\circ} \mathrm{C}\right)$. Boulenouar et al. [47] measured the impedance response of $\mathrm{Ni}$ wire grid electrodes on $\mathrm{YSZ}$ in various $\mathrm{CO}-\mathrm{CO}_{2}$ Ar gas mixtures and observed depressed semicircle responses for low $P_{O_{2}}$ and a more complex response, with two relaxation times, at high $P_{\mathrm{O}_{2}}$; they also observed scatter in their data in the low frequency regime. Lauvstad et al. [48] measured the impedance response of Pt and Ni point electrodes in a 33\% CO (balance Ar) gas mixture and compared with literature data on spectra for point electrodes in hydrogen and other gas mixtures. They concluded that the reaction mechanism on both Pt and Ni involves two adsorbed intermediates, $\mathrm{CO}_{\text {ads }}$ and $\mathrm{O}_{\mathrm{ads}}$, and that the rates of reaction of $\mathrm{CO}-\mathrm{CO}_{2}$ are slower than $\mathrm{H}_{2}-\mathrm{H}_{2} \mathrm{O}$ on Ni. Sukeshini et al. [49] microfabricated patterned Ni electrodes 
on YSZ electrolytes and made polarization and AC impedance measurements of the electrochemical oxidation of $\mathrm{H}_{2}, \mathrm{CO}$, and $\mathrm{CO} / \mathrm{H}_{2}$ mixtures. Their steady state measurements in 33.3\% CO in Ar had a higher activation overpotential at low current densities, but approached the overpotential for $\mathrm{H}_{2}$ at higher current densities. For their AC impedance measurements, they observed roughly double the area specific area resistance values in 33.3\% CO, compared to dry 33.3\% $\mathrm{H}_{2}$ in $\mathrm{Ar}$, under open circuit voltage conditions. Finally, they noted some scatter in their data at low frequencies, like Holtappels et al. [45] and Boulenouar et al. [47], indicating instability in the CO oxidation rates on $\mathrm{Ni}$.

\subsection{Proposed Research}

Unfortunately, the present conversion efficiencies for the solid oxide electrolysis of $\mathrm{CO}_{2}$ are estimated to be in the $20-40 \%$ range $[18,29]$. As noted in the previous section, the reaction kinetics of the electrochemical oxidation of $\mathrm{CO}$ or reduction of $\mathrm{CO}_{2}$ are poorly understood, and there is no consensus on the mechanism, or even the rate-limiting process, of these reactions on solid oxide electrodes.

As illustrated in Figure 1-2, it is generally believed that the carbon dioxide reduction reaction occurs at or very near the three phase boundary (TPB) where electronic-conducting electrode, ionic-conducting electrolyte, and gas phases meet; this argument is analogous to the proposed mechanism of oxygen reduction on solid oxide fuel cell cathodes [50]. This TPB area for a porous Pt electrode/YSZ electrolyte cell is limited to the actual interface where electrode and electrolyte meet. One way of 
enhancing the TPB area is to utilize alternative electrode materials that have both electronic and ionic conducting properties.

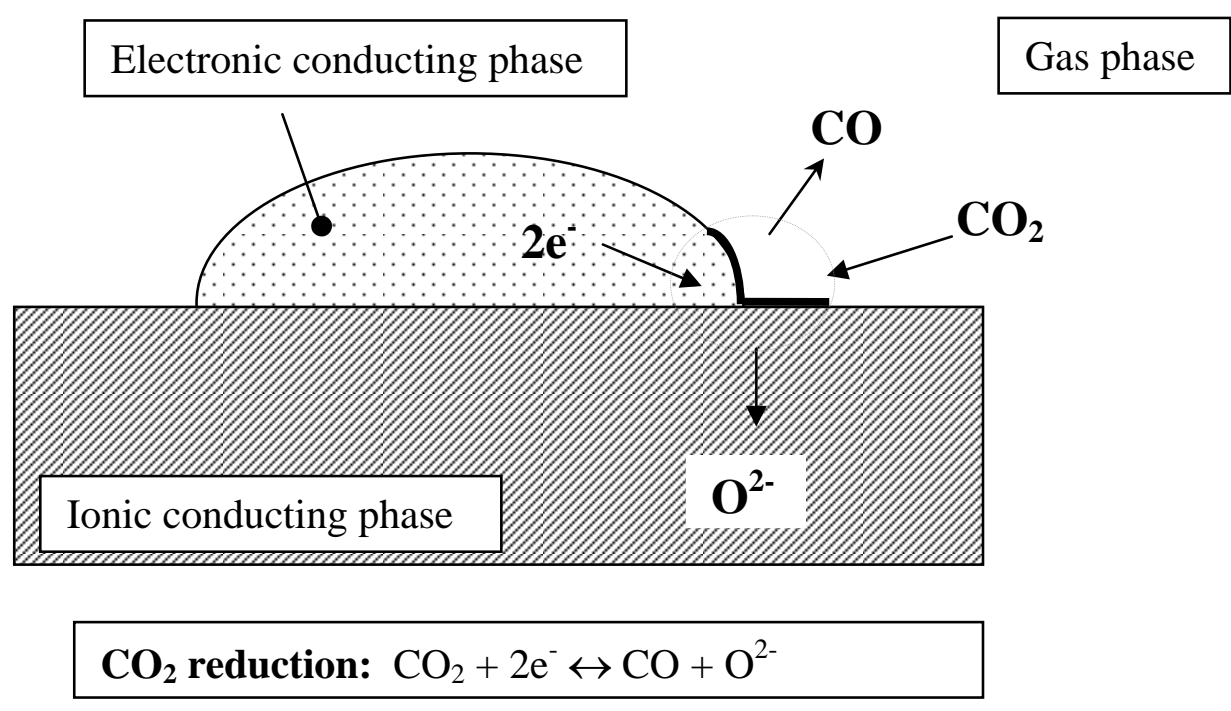

Figure 1-2. A qualitative illustration of the proposed mechanism of carbon dioxide reduction, i.e. $\mathrm{CO}_{2}+2 \mathrm{e}^{-} \leftrightarrow \mathrm{CO}+\mathrm{O}^{2-}$, on solid oxide cathodes. The three phase boundary (TPB) is highlighted with a dotted circle. The magnitude or extent of this TPB area is highly dependent on mixed conducting properties of the materials, electrocatalytic activity of the reaction on one or both of the material surfaces, surface diffusion of adsorbed species to the TPB, etc.

Figure 1-3 provides a qualitative graphic illustrating how the TPB area can be increased, comparing an electronic-only conducting electrode like porous platinum, with a mixed electronic-ionic conducting electrode material. Beginning in the mid-1960's, this approach was pursued as a key research thrust in electrode design for the solid oxide fuel cell (SOFC), starting with fuel cell anodes [43]. Today's state-of-the-art SOFC has 

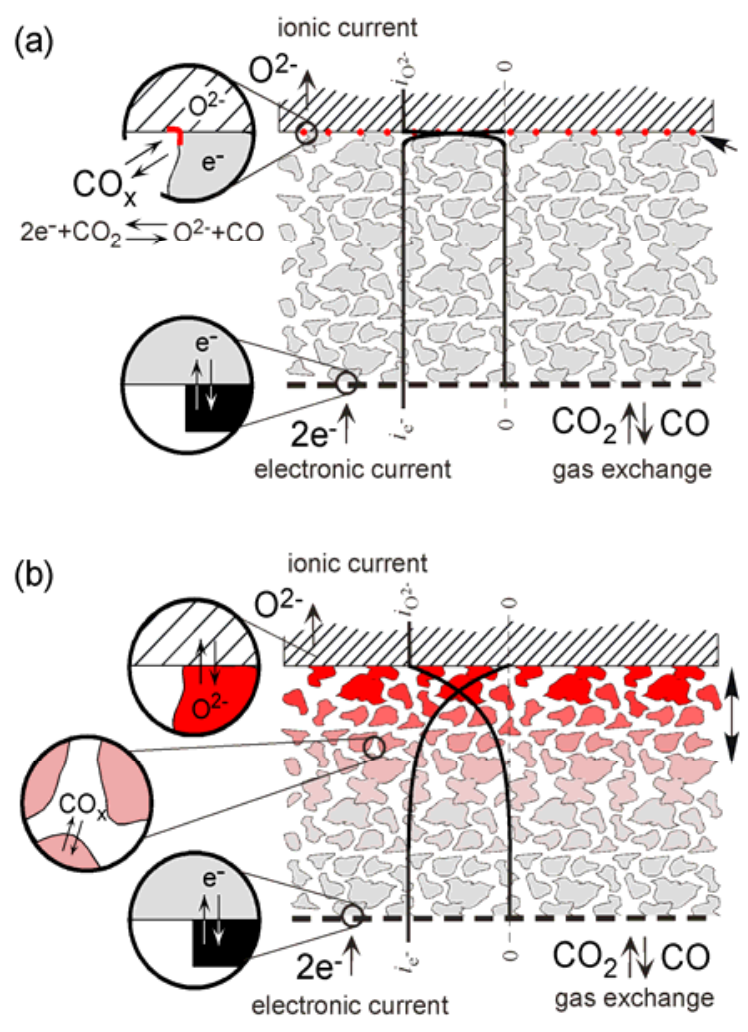

Figure 1-3. A qualitative illustration of (a) electronic-only conducting and (b) mixed-conducting porous electrode design. In (a), the three phase boundary (TPB) area is confined to the limited area where the electronic-only conducting electrode meets the ionic-conducting electrolyte. In (b), the TPB area is enhanced due to the mixed ionic-electronic conducting properties of the electrode; the reaction can occur over a larger extent of the internal surface area of the porous electrode, away from the electrode/electrolyte interface. The variation in shade of red color illustrates this variation in the $\mathrm{CO}_{2}$ reduction reaction rate through the thickness of the electrode. [See text for further explanation.] 
both an anode and cathode with mixed conducting properties, and illustrates one of the two approaches to achieving these electrode properties. As noted in the previous section, the commonly used SOFC anode is a Ni-YSZ ceramic-metallic (or cermet) electrode [51] consisting of $\mathrm{Ni}$ and YSZ particles sintered together in a porous matrix; the nickel phase acts as the electronic conductor while the YSZ is the oxygen ion conductor. The SOFC cathode of choice is also a composite electrode consisting of lathanum strontium manganite, $\mathrm{La}_{1-x} \mathrm{Sr}_{x} \mathrm{MnO}_{3}$, and YSZ (commonly abbreviated as LSM-YSZ).The other approach is to utilize a single phase electrode material that is both an electronic and ionic conductor, often referred to as a single phase mixed conductor; and, a number of pure and doped oxide materials do have these mixed conducting properties [52]. A good example is the lanthanum strontium cobalt iron oxide $\left(\mathrm{La}_{1-x} \mathrm{Sr}_{x} \mathrm{Co}_{1-y} \mathrm{Fe}_{y} \mathrm{O}_{3-\delta}\right.$ or LSCF) family of perovskite crystal oxides. Although these are known for their high electronic conductivity (i.e. electronic transference numbers approaching unity) [50], several also exhibit reasonably high oxygen ion conductivity; in particular, LSC $\left(\mathrm{La}_{1-x} \mathrm{Sr}_{x} \mathrm{CoO}_{3-\delta}\right)$ is a well-investigated mixed conductor and one of the first studied as an oxygen reduction cathode material for solid oxide fuel cells [50].

Another class of oxide materials is the rare-earth doped ceria family of fluorite crystal oxides $\left(\mathrm{M}_{x} \mathrm{Ce}_{1-x} \mathrm{O}_{2-\delta}\right.$, where $\mathrm{M}=\mathrm{Sm}, \mathrm{Gd}$, Sc, $\mathrm{La}, \mathrm{Mg}$, Ca, or $\left.\mathrm{Y}\right)$; these oxides have high ionic conductivity and are of practical interest as SOFC electrolytes. But under reducing atmospheres, they do exhibit electronic conductivity to varying degrees, making them also of interest as SOFC electrodes. 
The primary focus of this work is to investigate one of these single-phase mixedconducting materials, gadolinium-doped ceria, $\left(\mathrm{Ce}_{x} \mathrm{Gd}_{1-x} \mathrm{O}_{2-\delta}\right.$ or GDC), as a cathode for the reduction of carbon dioxide. Ceria is presently used as a catalyst support in catalytic converters in automobiles [53]. Some initial work with ceria as an SOFC anode for the direct oxidization of methane [54] and other hydrocarbons, suggests that it may also be electrocatalytically active for carbon dioxide reduction. The experimental technique of choice for this investigation will be electrochemical impedance spectroscopy.

Before delving into the experimental measurements on GDC electrodes, a slight digression is taken to perform a detailed computation of the relative error in our impedance measurements due to cell geometry (in Chapter 2). A summary of the material properties of GDC relevant to our investigation, with further rationale for the choice of GDC and doping level, is provided along with a description of the fabrication of our GDC electrodes and experimental setup (Chapter 3). A mathematical model of the CO- $\mathrm{CO}_{2}$ exchange reaction occurring on GDC porous electrodes is then described in detail (Chapter 4). Finally, the results of our experimental impedance data on GDC electrodes are discussed and fitted to the $\mathrm{CO}-\mathrm{CO}_{2}$ exchange model to draw preliminary conclusions on the transport and kinetic processes in the GDC electrode (Chapter 5). 


\section{References}

[1] National Aeronautics and Space Administration (NASA), The Vision for Space Exploration, NP-2004-01-334-HQ, NASA, Washington, D.C., 2004. See http://www.nasa.gov/mission_pages/exploration/main/index.html.

[2] TJ Kelly, Moon Lander: How we developed the Apollo Lunar Module, first ed., Smithsonian Institution Press, Washington, 2001.

[3] R Zubrin, S Price, Mars Sample Return mission utilizing in-situ propellant production; Final Report, NASA-CR-188371 (1995) 1-69.

[4] DP Thunnissen, D Rapp, CJ Voorhees, SF Dawson, CS Guernsey, A 2007 Mars Sample Return Mission utilizing in-situ propellant production, (1999) 1-20.

[5] R Odegard, J Keller, GA Landis, Oxygen generation and storage for a Mars sample return mission utilizing in-situ resources, AAIA 2007-6066, (2007).

[6] SJ Hoffman, DI Kaplan, Human Exploration of Mars:The Reference Mission of the NASA Mars Exploration Study Team, NASA Special Publication 6107, (1997) 3-101-3105.

[7] BG Drake, D Cooke, Reference Mission 3.0, Addendum to the Human Exploration of Mars: The Reference Mission of the NASA Mars Exploration Study Team, NASA internal publication EX13-98-036, (1998) 12-13.

[8] R Zubrin, The Case for Mars, Free Press, New York, NY, 1996.

[9] CP McKay, TR Meyer, PJ Boston, M Nelson, T Maccallum, O Gwynne, Utilizing Martian resources for life support, (1993) 819-843.

[10] AF Hepp, GA Landis, CP Kubiak, A Chemical Approach to Carbon Dioxide Utilization on Mars, (1993) 799-817.

[11] BK Breedlove, GM Ferrence, J Washington, CP Kubiak. A photoelectrochemical approach to splitting carbon dioxide for a manned mission to Mars, Materials and Design. 22 (2001) 577-584.

[12] CM Sanchez-Sanchez, V Montiel, DA Tryk, A Aldaz, A Fujishima. Electrochemical approaches to alleviation of the problem of carbon dioxide accumulation, Pure Appl. Chem. 73 (2001) 1917-1927.

[13] BS Singh, KR Sridhar, Research and Technology Needs for Chemical Processes and Operations on Mars, in: RG Galloway, S Lokaj (Eds.), Proceedings of the Sixth Annual Exposition on Engineering Construction and Operations in Space, American Society of Civil Engineers, Reston, VA (1998) 245-254. 
[14] KR Sridhar, R Foerstner. Regenerative solid oxide fuel cells for Mars exploration, J. Propul. Power. 16 (2000) 1105-1111.

[15] SH Jensen, Hogh, J. V. T., R Barfod, M Mogensen, High temperature electrolysis of steam and carbon dioxide, (2003) 204-215.

[16] CM Stoots, JE O'Brain, GL Hawkes, JS Herring, JJ Hartvigsen, High temperature co-electrolysis of $\mathrm{H}_{2} \mathrm{O}$ and $\mathrm{CO}_{2}$ for syngas production, 30 (Nov. 13-17 2006) Abstract \#418.

[17] GA Mills. Status and future opportunities for conversion of synthesis gas to liquid fuels, Fuel. 73 (1994) 1243-1279.

[18] SH Jensen, Solid oxide electrolyser cell, Riso-PhD-29(EN) (2006) 112-122.

[19] RH Socolow. Can we bury global warming? Sci. Am. 293 (2005) 49-55.

[20] An example of a start-up company developing a technology for removing $\mathrm{CO}_{2}$ from the atmosphere can be found at http://www.grestech.com/ (K Lackner, Global Research Technologies LLC, 2008).

[21] L Elikan, DH Archer, RL Zahradnik. Oxygen Regeneration in Solid Electrolyte Batteries: Fundamental Considerations, AICHE Chemical Engineering Progress Series: Aerospace Life Support. 62 (1966) 29-37.

[22] HW Chandler, FZ Pollara. Oxygen Regeneration in a Solid Electrolyte System, AICHE Chemical Engineering Progress Series: Aerospace Life Support. 62 (1966) 38-42.

[23] L Elikan, JP Morris, Solid oxide electrolyte system for oxygen regeneration, NASA CR-1359 (1969) 1-166.

[24] L Elikan, JP Morris, CK Wu, Development of a solid oxide electrolyte carbon dioxide and water reduction system for oxygen recovery, NASA CR-2014 (1972) 1-169.

[25] RL Ash, WL Dowler, G Varsi. Feasibility of Rocket Propellant on Mars, Acta Astronautica. 5 (1978) 705-724.

[26] ML Stancati, JC Niehoff, WC Wells, RL Ash, Remote Automated Propellant Production: a New Potential for Round Trip Spacecraft, AIAA 79-0906. (1979) 262-270.

[27] R Richter, Basic Investigation into the Production of Oxygen in a Solid Electrolyte, AIAA-81-1175, (1981) 1-14.

[28] SC Crow, The MOXCE Project: New Cells for Producing Oxygen on Mars, $33^{\text {rd }}$ AIAA/ASME/SAE/ASEE Joint Propulsion Conference and Exhibit, AAIA 97-2766, (1997).

[29] D Rapp, A review of Mars ISPP technology, JPL D-15223 (1998) 60-64. 
[30] BC Sales, JE Turner, MB Maple. Oscillatory oxidation of CO over Pt, Pd, and Ir catalysts: Theory, Surface Science. 114 (1982) 381-394.

[31] VA Burrows, S Sundaresan, YJ Chabal. Real-time study of self-sustained oscillations on the CO oxidation rate on Pt, J. Vac. Sci. Technol. A. 5 (1987) 801-804.

[32] HJ Freund, MW Roberts. Surface chemistry of carbon dioxide, Surface Science Reports. 25 (1996) 225-273.

[33] SV Karpachev, AE Zupniik, SF Pal'guev. Polarization of carbon monoxide electrodes on platinum in a solid zirconia lime electrolyte, Electrochim. Acta. 9 (1964) 1681-1685.

[34] TH Etsell, SN Flengas. Overpotential behavior of stabilized zirconia solid electrolyte fuel cells, J. Electrochem. Soc. 118 (1971) 1890-1900.

[35] J Mizusaki, H Tagawa, Y Miyaki, S Yamauchi, K Fueki, I Koshiro, et al. Kinetics of the Electrode-Reaction at the $\mathrm{CO}-\mathrm{CO}_{2}$, Porous Pt/stabilized Zirconia Interface, Solid State Ionics. 53-56 (1992) 126-134.

[36] IS Metcalfe, S Sundaresan. Oxygen Transfer between Metals and Oxygen-Ion Conducting Supports, AICHE Journal. 34 (1988) 195-208.

[37] IS Metcalfe. Carbon Monoxide Oxidation over Platinum Supported on Yttriastabilized Zirconia, Brit. Ceram. Proc. 43 (1988) 139-150.

[38] G Tao, KR Sridhar, CL Chan. Study of carbon dioxide electrolysis at electrode/electrolyte interface: Part I. Pt/YSZ interface, Solid State Ionics. 175 (2004) 615-619.

[39] G Tao, KR Sridhar, CL Chan. Study of carbon dioxide electrolysis at electrode/electrolyte interface: Part II. Pt-YSZ cermet/YSZ interface, Solid State Ionics. 175 (2004) 621-624.

[40] G Lauvstad, R Tunold, S Sunde, Oxidation of carbon monoxide on strontium ferrate, 95-1 (1995) 731-740.

[41] JY Park, ED Wachsman. Lower temperature electrolytic reduction of $\mathrm{CO}_{2}$ to $\mathrm{O}_{2}$ and CO with high-conductivity solid oxide bilayer electrolytes, J. Electrochem. Soc. 8 (2005) A1654-A1659.

[42] F Bidrawn, G Kim, G Corre, JTS Irvine, JM Vohs, RJ Gorte. Efficient Reduction of $\mathrm{CO}_{2}$ in a Solid Oxide Electrolyzer, Electrochem. Solid-State Lett. 11 (2008) B167-B170.

[43] A Atkinson, S Barnett, RJ Gorte, Irvine, J. T. S., AJ Mcevoy, M Mogensen, et al. Advanced anodes for high-temperature fuel cells, Nature Materials. 3 (2004) 17-27. 
[44] RJ Aaberg, R Tunold, S Tjelle, R Odegard, Oxidation of $\mathrm{CO}$ and $\mathrm{H}_{2}$ on Ni/YSZ cermet electrodes, in: Poulsen FW, Bonanos N, Linderoth S, Mogensen M, ZachauChristiansen B (Eds.), High Temperature Electrochemistry: Ceramics and Metals, Proceedings of the 17th Riso International Symposium of Materials Science, Roskilde, Denmark ed., Riso National Laboratory, Roskilde, Denmark, 1996, pp. 511-516.

[45] P Holtappels, De Haart, L. G. J., U Stimming, IC Vinke, M Mogensen. Reaction of CO/CO2 gas mixtures on Ni-YSZ cermet electrodes, J.Appl.Electrochem. 29 (1999) 561568.

[46] Y Matsuzaki, I Yasuda. Electrochemical oxidation of $\mathrm{H} 2$ and $\mathrm{CO}$ in a $\mathrm{H}_{2}-\mathrm{H}_{2} \mathrm{O}-\mathrm{CO}-$ $\mathrm{CO}_{2}$ system at the interface of a Ni-YSZ cermet electrode and YSZ electrolyte, J. Electrochem. Soc. 147 (2000) 1630-1635.

[47] FZ Boulenouar, K Yashiro, M Oishi, A Kaimai, Y Nigara, T Kawada, et al., Electrochemical oxidation of $\mathrm{CO}$ in a $\mathrm{CO}-\mathrm{CO}_{2}$ system a the interface of Ni grid electrode/YSZ electrolyte, in: Yokokawa H, Singhal SC (Eds.), Proceedings of the Seventh International Symposium on Solid Oxide Fuel Cells, SOFC VII, PV2001-16, Pennington, NJ ed., The Electrochemical Society, Pennington, NJ, 2001, pp. 759-768.

[48] GO Lauvstad, R Tunold, S Sunde. Electrochemical oxidation of CO on Pt and Ni point electrodes in contact with an yttria-stabilized zirconia electrolyte - II. Steady-state and impedance measurements, J.Electrochem.Soc. 149 (2002) E506-E514.

[49] AM Sukeshini, B Habibzadeh, BP Becker, CA Stoltz, BW Eichhorn, GS Jackson. Electrochemical oxidation of $\mathrm{H}_{2}$, $\mathrm{CO}$, and $\mathrm{CO} / \mathrm{H}_{2}$ mixtures on patterned $\mathrm{Ni}$ anodes on YSZ electrolytes, J.Electrochem.Soc. 153 (2006) A705-A715.

[50] SB Adler. Factors governing oxygen reduction in solid oxide fuel cell cathodes, Chem. Rev. 104 (2004) 4791-4843.

[51] M Mogensen, S Primdahl, MJ Jorgensen, C Bagger. Composite Electrodes in Solid Oxide Fuel Cells and Similar Solid State Devices, Journal of Electroceramics. 5 (2000) $141-152$.

[52] I Riess, Electrochemistry of Mixed Ionic-Electronic Conductors (chapter 7), The CRC Handbook of Solid State Electrochemistry, first edition ed., CRC Press, Boca Raton, FL, 1997, pp. 223-268.

[53] M Shelef, GW Graham, RW McCabe, Ceria and other oxygen storage components in automotive catalysts, in: Trovarelli A (Ed.), Catalysis by Ceria and Related Materials, vol. 2, Imperial College Press, London, 2002, pp. 343-365.

[54] EP Murray, T Tsai, SA Barnett. A direct-methane fuel cell with a ceria-based anode, Nature. 400 (1999) 649-651. 


\section{CHAPTER 2}

FINITE ELEMENT ANALYSES OF REFERENCE ELECTRODE PLACEMENT ON THICK ELECTROLYTES TO QUANTIFY THE EFFECT ON

ELECTROCHEMICAL IMPEDANCE SPECTROSCOPY (EIS) MEASUREMENTS

\subsection{Introduction}

In the experimental electrochemical studies, cell geometry along with placement of reference electrodes has a significant impact on the precision of electrochemical measurements. In particular, solid state electrochemical systems limit the flexibility in cell design due to solid state electrolytes typically having much lower conductivities than aqueous electrolytes, along with additional fabrication challenges. It is common for workers in solid state electrochemistry to utilize thick electrolytes when performing polarization and electrochemical impedance spectroscopy (EIS) measurements to isolate the electrode of interest. In this chapter, a perspective on thick pellet geometry effects on the precision of EIS measurements is provided; in particular, a computational analysis is presented to estimate the error induced into the EIS measurements by the particular thick electrolyte cell geometry used for the experimental measurements in this work. 


\subsubsection{Background}

A number of workers typically use their own "custom" electrochemical cell designs based on their lab affiliation or particular type of research they are performing [13]. Often, they do not report an estimate of the error in their electrochemical measurements potentially induce by cell geometry or reference electrode location, or any optimization analysis they've performed to minimize measurement error. But, a number of researchers have performed analyses or experiments, that indicate the measurement error may be substantial [4-7]. For this reason, it is worthwhile to perform a computational analysis of any cell geometry being considered, to ascertain any errors this geometry may induce, to aid in choosing a cell geometry, or to optimize the dimensions of a chosen cell geometry to minimize polarization and impedance measurement errors.

It is well-known from aqueous electrochemistry that reference electrode placement can distort polarization measurements. Newman [4] determined the current distribution across a rotating disk electrode below the limiting current and showed that the ohmic potential drop is non-uniform across the disk due to non-uniform current density.

For aqueous electrolyte systems, the Luggin capillary reference electrode [8] was developed to overcome electrolyte resistance in dilute aqueous solutions or poorly conductive non-aqueous electrolytes [9]. As shown in Figure 2-1, a Luggin reference electrode is designed to measure the potential of the electrolyte solution close to the working electrode while minimally disturbing the current density distribution between 
working and counter electrodes. The diameter of a Luggin capillary is on the order of 1 $\mathrm{mm}$ [8], small relative to the working electrode area of one to several $\mathrm{cm}^{2}$ used in typical laboratory scale aqueous electrochemistry systems.

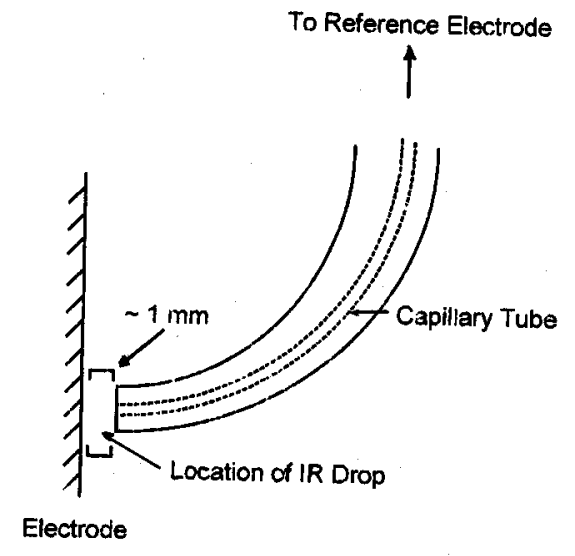

Figure 2-1. Diagram of a Luggin capillary commonly used as a reference electrode in aqueous electrochemistry studies. From reference [10].

In particular, electrochemical systems with thin electrolytes (thin being the electrolyte thickness $<<$ electrode length scale) are problematic, in that it is difficult to place the reference electrode close to the working electrode. In most solid state electrochemical systems, especially devices like battery cells and fuel cells, the electrolyte thickness is on the order of microns, fitting this definition. For practical reasons, researchers working with these systems place the reference electrode in some manner on the planar electrolyte adjacent to the working electrode; a few have investigated the effect of this reference electrode placement, on polarization and impedance measurements. 
Nagata et al. [5] performed DC polarization measurements on disc-shaped (cylindrical geometry) and rectangular (Cartesian geometry) planar cells and concluded that accurate overpotentials can most accurately be measured on disc-shaped planar cells with a symmetrically-positioned anode and cathode, and the reference electrode positioned far from both working and counter electrodes. Adler et al. [7] quantified this further by performing finite element analyses on both thin and thick electrolyte geometries, and showed that positioning the reference electrode at least 3 three electrolyte thicknesses beyond the edge of the active electrode(s) will minimize the drift in potential of the reference electrode for $\mathrm{AC}$ measurements.

Kato et al. [6] demonstrated with both computational modeling and impedance experiments on cylindrical disk geometry cells, that parasitic arcs can be introduced into the impedance spectra of a working electrode, and these can be attributed to counter electrodes with different diameters or different capacitances than the working electrode, more specifically, differing capacitances at the electrode/electrolyte interface. They note that the measurement error can be reduced by assuring identical size and alignment of the working electrodes on a symmetric cell, and closely matching the performance, i.e. the capacitance, of the counter-electrode with the working electrode.

Hsieh et al. [11-14] looked at reference electrode placement in thick pellet geometries and made both impedance and DC polarization measurements. In particular, reference [13] details computer simulations and experimental results for thick electrolyte cylindrical pellets with a reference electrode hole drilled from the counter-electrode face. 
They found this geometry accurately measures both working and counter-electrode impedances, as long as the reference electrode hole diameter is small compared to the electrode diameter.

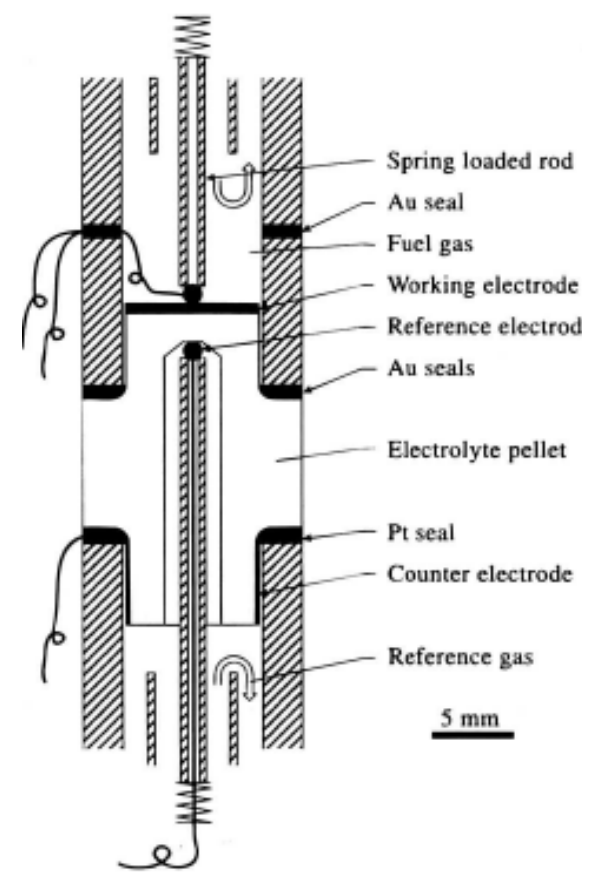

Figure 2-2. Diagram of the "Risø pellet" cell. From reference [1].

Winkler et al. [15] showed that deviations can occur due to improper placement of reference electrodes for DC measurements. They simulated the electrode polarization resistances of both planar and pellet geometries at the high $(\omega \rightarrow \infty)$ and low $(\omega \rightarrow 0)$ frequency limits - corresponding to primary current and secondary current distributions respectively - in the electrolyte. They concluded that for thin planar three-electrode cell geometries, with improperly-placed reference electrodes, can cause large errors in measured electrode polarization resistances, while pellet and other thick electrolyte geometries are less susceptible to these errors. Specifically, their computational results 
show their in-house developed "Risø pellet" geometry can measure electrode polarization resistances with less than $5 \%$ error.

Adler [16] performed more extensive finite element analyses, simulating typical impedance experiments by sweeping the frequency spectrum, and showed that 1) AC impedance measurements can be distorted on thin electrolytes due to even slight misalignment of the working and counter-electrodes, and 2) it is nearly impossible to find an ideal reference electrode position that minimizes these distortions for thin planar geometries. He provided two possible solutions, 1) thick, i.e. pellet geometries, and 2) microelectrodes on thin electrolytes. He performed a detailed analysis on a core-drilled cylindrical pellet cell and showed the impedance response is slightly distorted, but in a linear predictable manner. He demonstrated the second solution, a microelectrode, can produce an accurate impedance response, and noted this point-type electrode is of particular promise, as it can be fabricated utilizing the same standard fabrication methods for thin electrolyte planar cells, thus more closely matching experimental results for a full solid oxide electrochemical cell.

Coffey et al. [9] fabricated Pt/YSZ/Pt cells with a reference electrode embedded in a thin ( $2 \mathrm{~mm}$ thick) electrolyte, as opposed to a drilled hole commonly used for thick pellet geometries. Their reference electrode, which they refer to as a Luggin probe, consists of a thin (750 $\mu \mathrm{m}$ diameter) Pt wire insulated from the electrolyte with an aluminum oxide coating, and embedded in the YSZ electrolyte perpendicular to the working electrode. This type of reference electrode virtually eliminates the reference 
electrode "shielding" problem, but requires additional fabrication steps, including the addition of a thin porous layer of YSZ at the working electrode/electrolyte interface, necessary in order to provide gaseous oxygen to the reference electrode. They performed cyclic voltammetry and AC impedance measurements and found the reference electrode is stable, maintaining a constant potential, but they did observe variability between reference electrodes, which they attribute to variations in working electrode composition and thickness of the intermediate porous YSZ layer. In addition, this thin porous layer of YSZ may potentially affect the performance of this cell relative to a planar cell without the porous layer. Experimental measurements on the cell demonstrate that the reference electrode is stable, but gas concentration gradients may cause drift of the reference electrode potential, depending on the current flux.

Most recently Rutman and Riess [17-19] performed a series of detailed computational analyses on thin electrolyte cells, proposing a cell configuration with an extension in the electrolyte for mounting the reference electrode, to avoid a non-uniform current density distribution. And separately, they developed two methods of addressing measurement errors due to misaligned electrodes on thin cylindrical or planar geometry cells, 1) calculate calibration factors as a function of the electrode offset, or 2) use an averaging procedure of the potential of two or more reference electrodes placed on the periphery of the electrolyte. 


\subsubsection{Thick electrolyte or "pellet" cells}

The thick electrolyte, as alluded to above, offers a potentially good platform for making precision electrochemical measurements, in that a thick electrode allows the working and counter-electrodes to be positioned relatively far from each other, and adds flexibility in the location of the reference electrode(s). It is also fairly straightforward and simple to fabricate; an example of typically used fabrication procedures is described in the experimental setup in Chapter 3. But there are a few disadvantages to this type of cell.

A thick electrolyte, due to increased resistivity, will limit the current density as which measurements can be made, thus restricting the range of operation, and possibly the optimum performance point(s) for a particular cell chemistry. Also, operating at high current densities will increase Joule heating of a thick electrolyte cell, potentially affecting cell temperature under certain measurement conditions.

The thick electrolyte cell typically utilizes different fabrication techniques, coldpressing, as opposed to tape casting or screen-printing used for thin electrolytes. This may result in an electrolyte surface with different electrode/electrolyte interfacial properties, or in the bulk of the electrolyte, different electronic-ionic transport properties, in comparison to a thin electrolyte cell.

A thick electrolyte can potentially increase instrumentation errors for electrochemical impedance spectroscopy (EIS) measurements. If the ratio of electrode 
polarization resistance to electrolyte resistance is low, the EIS signal-to-noise ratio will decrease depending on the instrumentation current-sensing standard resistor chosen; this can effect the precision of impedance measurements for small phase angles at high frequencies [20].

Finally, thick electrolytes are not feasible for some fuel cell chemistries; in particular, the polymer electrolyte membrane (PEM) fuel cell can not be fabricated with a thick electrolyte. The reason for this is the PEM electrolyte performance is also affected by water balance and gas cross-over variations, both difficult to control in a thick polymer-type membrane [21].

\subsubsection{Microelectrode cells}

The term, microelectrode cell, is typically applied to electrodes with dimension in the range of micrometers, and more recently, down to the nanometer range. True microelectrodes have been used extensively in fundamental studies in liquid state electrochemistry to avoid altering the concentration of electroactive species in the bulk electrolyte [22] (p. 156). Adler [16] and Hashibon et al. [23] have proposed similar micro-electrode designs consisting of an interdigitated array of narrow working electrode strips (to increase electrode area) in parallel with a similar array of reference electrode strips, and both provided numerical analysis results to quantify the reduced measurement error afforded by these concepts. Dunyushkina et al. [24] report an initial experimental study of a micro-array electrode, demonstrating the microelectrode-on-a-thin-electrolyte concept proposed by Adler [16]; their work demonstrated the feasibility, but they note it 
does require some additional fabrication procedures beyond those in a "production-like" thin electrolyte cell; in particular, the application of a dielectric coating to isolate the electrode from the adjacent reference electrode.

\subsubsection{Objectives of the cell geometry analyses}

In the experimental portion of this work, the thick electrolyte pellet cell geometry, shown in Figure 2-3, is used for the experimental measurements on GDC electrodes, referred to as the baseline geometry cell. In this chapter, a detailed computational simulation of the impedance of this baseline geometry cell is performed, utilizing finite element analyses to predict the primary current distribution in the pellet electrolyte and the resulting measurement errors due to this cell geometry. In addition, some parametric analyses are performed to show how several variations in the cell geometry, misaligned electrodes, and non-linear or asymmetric electrodes, increase or reduce these measurement errors.

\subsection{Theory and Computational Model Formulation}

The details of this mathematical formulation were discussed in a previous work [16]. In this section, a summary of the theory is provided along with the additional assumptions and enhancements to the model for this particular analysis. Because the direct implementation of a model into commercial mathematical software is not always straightforward, the details of implementing this computational model into the finite element software, COMSOL Multiphysics ${ }^{\mathrm{TM}}$ (COMSOL AB), are presented in Appendix 
A. In addition, a sample MATLAB (The MathWorks ${ }^{\mathrm{TM}}$ ) script (or M-file) for running the simulation and post-processing the results is available to the reader in Appendix $\mathrm{H}$.

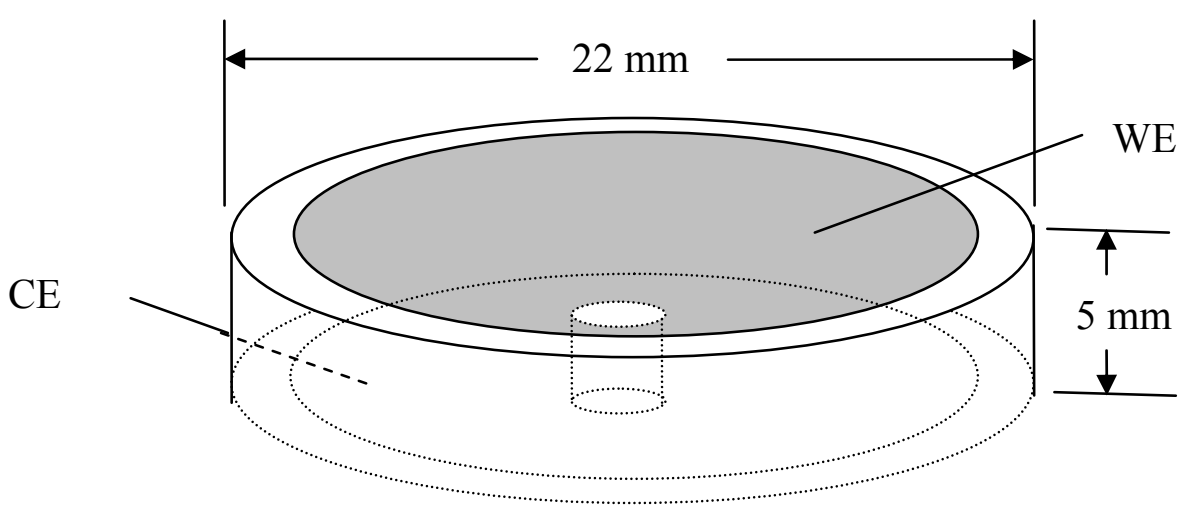

(a)

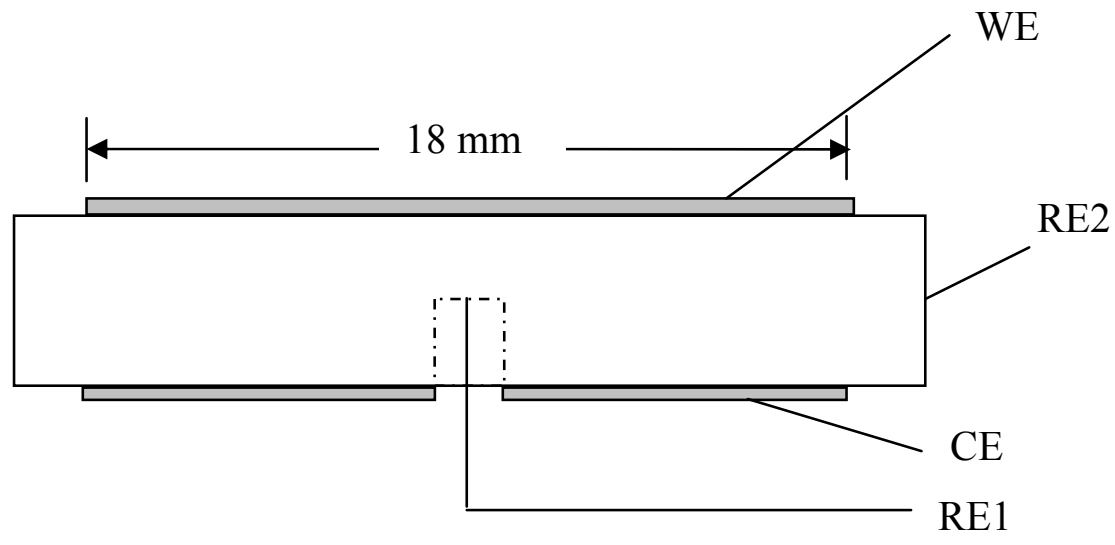

(b)

Figure 2-3. Diagram of the baseline thick pellet cell geometry. (a) drawing of pellet cell showing overall dimensions and locations of working electrode (WE) and counter-electrode (CE). (b) cross-section of cylindrical pellet cell showing diameter of electrodes and reference electrode (RE1 and RE2) locations. 


\subsubsection{Governing equation for the electrolyte pellet model}

For our computational problem, the computational domain is defined as the axisymmetric "slice" of the electrolyte pellet geometry we chose for our experimental research, shown in Figure 2-4. A material balance on the conducting species, oxygen ions, can be expressed as:

$$
\frac{\partial c_{i}}{\partial t}=-\nabla \cdot \mathbf{N}_{i}+R_{i}
$$

Inside the electrode, there is no reaction of conducting species, i.e. $R_{i}=0$. Assuming no convection or concentration gradients, the flux of the conducting species, $\mathbf{N}_{i}$, is reduced to migration, or, expressed in terms of the current density:

$$
\mathbf{i}=F \sum_{i} z_{i} \mathbf{N}_{i}=F^{2} \nabla \Phi \sum_{i} z_{i}^{2} u_{i} c_{i}
$$

where $\Phi$ is the potential inside the electrolyte and $u_{i}$ is the mobility of the conducting species. 


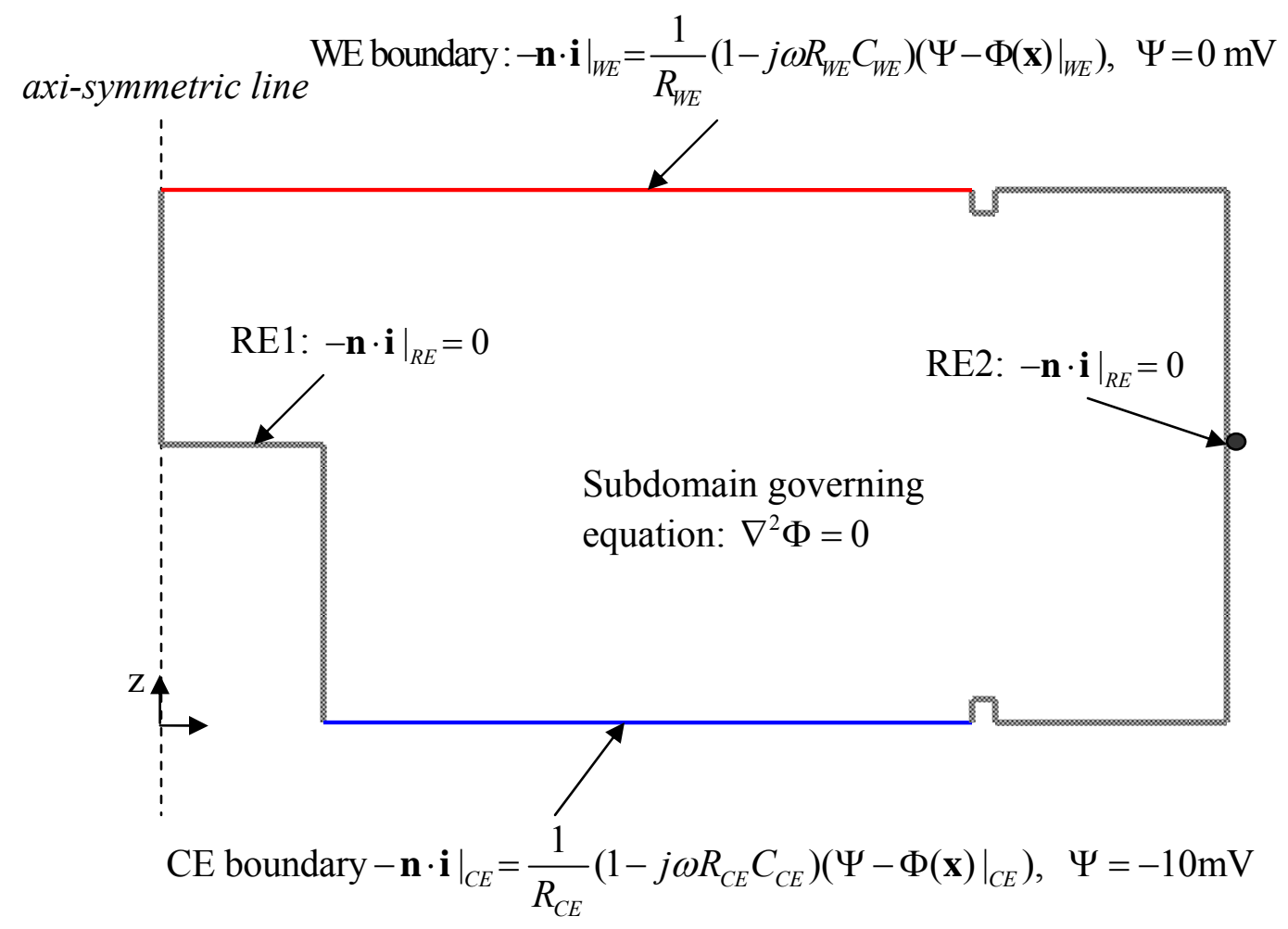

Boundary is insulated, i.e. $-\left.\mathbf{n} \cdot \mathbf{i}\right|_{\text {insulated }}=0 \quad$ Drawing is not to scale.

Figure 2-4. Diagram of the 2D axisymmetric pellet geometry defining the computational subdomain and associated boundary conditions. The reference electrode position is treated as an insulated boundary condition to reflect no current flow. See text for a more detailed description and derivation of the working and counter-electrode boundary conditions. The small 'notches' -- enlarged for illustration purposes -- are placed at the edge of the working and counter-electrodes to keep the computed current density finite at these points (described further in the text). The 3D pellet geometry conditions are defined similarly, but on a "half-pellet" computational subdomain to reflect the less symmetric misaligned working electrode condition. 
Equation (2-2) is an expression of Ohm's Law:

$$
\mathbf{i}=-\kappa \nabla \Phi
$$

where $\kappa\left(=F^{2} \sum_{i} z_{i}^{2} u_{i} c_{i}\right)$ is the electrolyte ionic conductivity. Assuming the

electroneutrality condition applies inside the electrolyte:

$$
\nabla \cdot \mathbf{i}=0
$$

Combining Equations (2-3) and (2-4), the governing equation for conduction in the electrolyte is obtained in terms of $\Phi$ :

$$
-\nabla \cdot(\kappa \nabla \Phi)=0
$$

The electrolyte potential, $\Phi$, is assumed to be separable into $\tilde{\Phi}(\mathbf{x})$, a function of position alone, and $\hat{\Phi}(t)$, a function of time alone. This is commonly done in electrode models for linear impedance response analyses [25]:

$$
\Phi(\mathbf{x}, t)=\tilde{\Phi}(\mathbf{x}) \hat{\Phi}(t)=\tilde{\Phi}(\mathbf{x}) e^{-j \omega t}
$$

where: $j=\sqrt{-1}$

$\omega=2 \pi f=$ angular (or radial) frequency

$\mathbf{x}=$ is a position vector for the scalar $\Phi$ 
For the 2D analyses, specifically for cases where the WE and CE are properly aligned, Equation (2-5) was transformed to cylindrical coordinates:

$$
\frac{1}{r} \frac{\partial}{\partial r}\left(\kappa r \frac{\partial \Phi}{\partial r}\right)+\frac{1}{r^{2}} \frac{\partial}{\partial \theta}\left(\kappa \frac{\partial \Phi}{\partial \theta}\right)+\frac{\partial}{\partial z}\left(\kappa \frac{\partial \Phi}{\partial z}\right)=0
$$

Equation (2-7) can be reduced to a 2D problem due to axisymmetry, i.e. the solution is independent of $\theta$. Also, Equation (2-7) is multiplied through by $r$ to avoid a singularity in the $\frac{\partial}{\partial r}$ term for the solution as $r \rightarrow 0$ near the axisymmetric line:

$$
\frac{\partial}{\partial r}\left(\kappa r \frac{\partial \Phi}{\partial r}\right)+\frac{\partial}{\partial z}\left(\kappa r \frac{\partial \Phi}{\partial z}\right)=0
$$

For the 3D analyses, Equation (2-5) was transformed to Cartesian coordinates:

$$
\frac{\partial}{\partial x}\left(\kappa \frac{\partial \Phi}{\partial x}\right)+\frac{\partial}{\partial y}\left(\kappa \frac{\partial \Phi}{\partial y}\right)+\frac{\partial}{\partial z}\left(\kappa \frac{\partial \Phi}{\partial z}\right)=0
$$


Note that for this analysis, the temperature is assumed constant and the ionic conductivity in the electrolyte is assumed to be isotropic ${ }^{*}$, so Equation (2-5) actually reduces to Laplace's equation:

$$
\nabla^{2} \Phi=0
$$

But the slightly more general form in Equations (2-8) and (2-9) was required for implementation in the finite element software. See Appendix A for further details.

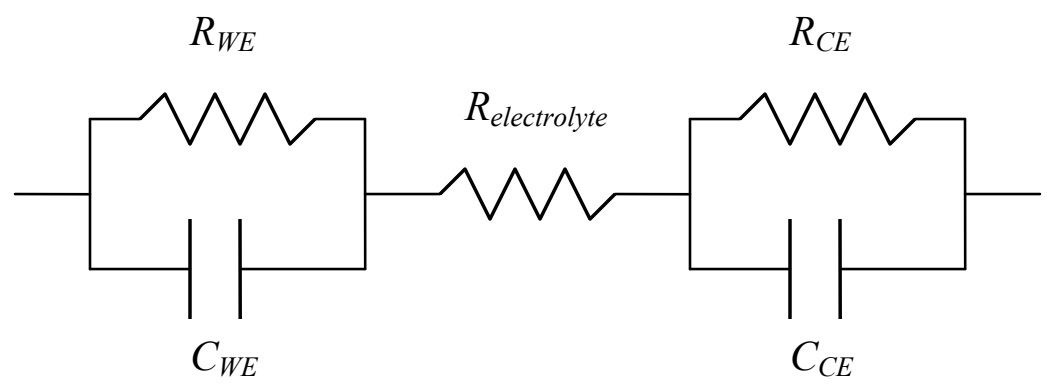

Figure 2-5. Electrical analog of cell model.

${ }^{*}$ Most solid oxide electrolytes are polycrystalline due to the typical fabrication methods of tape casting for thin electrolytes, or die casting for thick electrolyte pellet-type cells; hence, the ionic conductivity property is considered isotropic. 


\subsubsection{Boundary conditions}

In order to simulate a linear frequency response, the model of an ideal RC electrode was chosen for the working and counter-electrodes, consisting of an equivalent circuit of a resistor and capacitor in parallel as shown in Figure 2-5. This model is applied as a boundary condition to simulate the impedance response of a true electrode.

The current density is defined on the boundary of the working electrode (WE) as follows:

$$
\left.i(\mathbf{x}, t)\right|_{W E}=-\left.\mathbf{n} \cdot \kappa \nabla \Phi(\mathbf{x}, t)\right|_{W E}=\left(\frac{1}{R_{W E}}+C_{W E} \frac{\partial}{\partial t}\right)\left(\Psi(t)-\left.\Phi(\mathbf{x}, t)\right|_{W E}\right)
$$

where: $i=$ current density

$R_{W E}=$ area-specific resistance of the working electrode $\left(\right.$ in $\Omega-\mathrm{cm}^{2}$ )

$C_{W E}=$ area-specific capacitance of the working electrode $\left(\right.$ in $\left.\frac{\mathrm{F}}{\mathrm{cm}^{2}}\right)$

$\Psi=$ the electrode potential just outside the electrode/electrolyte interface

$\mathbf{x}=$ a position vector for the scalar function, $\Phi$

Similar to $\Phi, \Psi$ is also assumed to be separable:

$$
\Psi(t)=\tilde{\Psi} e^{-j \omega t}
$$

where $\tilde{\Psi}=$ a constant

Substituting Equations (2-6) and (2-12) into Equation (2-11) and simplifying:

$$
\left.i(\mathbf{x})\right|_{W E}=-\left.\kappa \mathbf{n} \cdot \nabla \Phi(\mathbf{x})\right|_{W E}=\frac{1-j \omega R_{W E} C_{W E}}{R_{W E}}\left(\Psi-\left.\Phi(\mathbf{x})\right|_{W E}\right)
$$




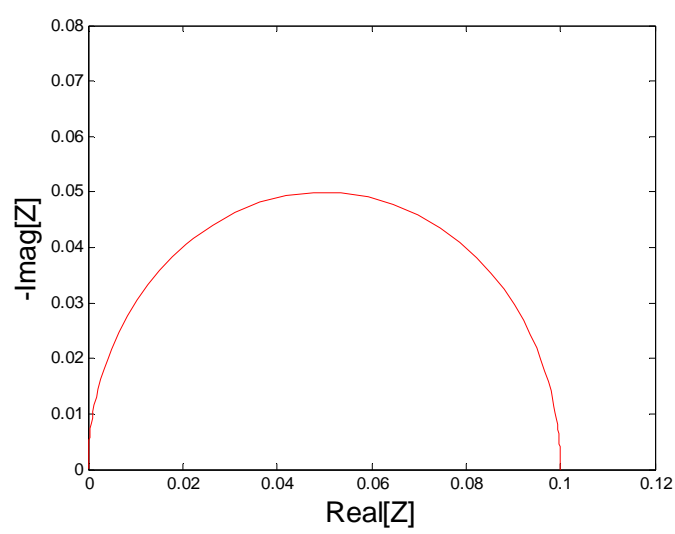

(a)

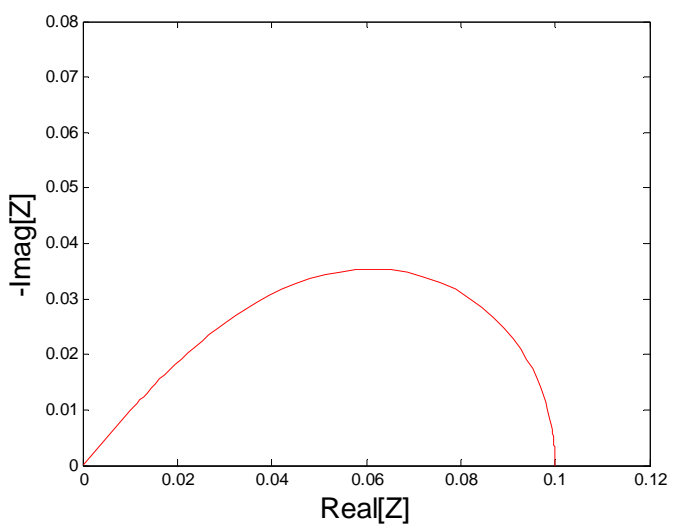

(b)

Figure 2-6. Nyquist plots for samples of the impedance responses for the two boundary condition electrode models used for the analyses in this chapter. (a) Ideal linear electrode assuming a resistor and capacitor in parallel model in Equation (2-11) with $R_{W E}=0.1, C_{W E}=1.0$; (b) ideal non-linear electrode model in Equation (2-16) with $R_{W E}=0.01, C_{W E}=5, \alpha_{W E}=0.50$.

The impedance response of this ideal RC electrode boundary condition is shown in Figure 2-6 (a). A similar boundary condition is applied for the counter-electrode (CE). The reference electrode positions are treated as insulated boundary conditions (i.e. no current flow).$^{\dagger}$

\footnotetext{
$\dagger$ Negligible current is drawn on a reference electrode probe by the instrumentation’s high impedance voltmeter during a polarization or impedance measurement.
} 


\subsubsection{AC impedance derived from the current density solution}

The finite element solution of this problem determines $\Phi(\mathbf{x})$ and $\nabla \Phi(\mathbf{x})$ in the computational domain. The simulated AC impedance response for the $\mathrm{WE}, Z_{W E}$, can be calculated by evaluating $\Phi(\mathbf{x})$ at the appropriate reference electrode position, along with the total current density of the WE, evaluated by integrating $i(\mathbf{x})=\mathbf{n} \cdot \kappa \nabla \Phi(\mathbf{x})$ along the WE boundary:

$$
Z_{W E}=\frac{\left.\Psi e^{-j \omega t}\right|_{W E}-\left.\Phi(\mathbf{x}) e^{-j \omega t}\right|_{R E}}{\left.\int_{A_{W E}} i(\mathbf{x})\right|_{W E} e^{-j \omega t} d A_{W E}}=\frac{\left.\Psi\right|_{W E}-\left.\Phi(\mathbf{x})\right|_{R E}}{\left.\int_{A_{W E}} i(\mathbf{x})\right|_{W E} d A_{W E}}
$$

For the reference electrode at the base of the hole in the pellet (designated as RE1), an area-averaged potential is calculated by numerically integrating over the RE1 boundary, and dividing by the area of the RE1 boundary, i.e. the $\left.\Phi(\mathbf{x})\right|_{R E}$ term in Equation (2-14) is substituted with the following:

$$
\Phi_{R E 1}^{a v g}=\frac{\left.\int_{A_{R E}} \Phi(\mathbf{x})\right|_{R E 1} d A}{A_{R E 1}}
$$

For the reference electrode at the edge of the pellet, RE2, this second term is the potential, $\left.\Phi(\mathbf{x})\right|_{R E 2}$, evaluated at a single point, i.e. the midpoint of the outer edge of the pellet as shown in Figure 2-2. For the 3D analyses, the RE2 electrode potential is 
evaluated at a number of edge points at the circumference of the $1 / 2$ pellet computational domain, and numerically averaged.

\subsubsection{Model for the non-linear electrode boundary condition}

The described impedance model for both the working and counter-electrode is a ideal linear response model. A question of whether a non-linear impedance response, more characteristic of the response of a real electrode, is distorted by the cell geometry and placement of reference electrodes. In order to investigate this aspect, a non-linear model was constructed by modifying the WE or CE boundary condition with an empirical-based exponent. An impedance model similar to this was used by Wang and Norwick [25] to fit non-linear data for porous platinum electrodes. ${ }^{\ddagger}$ The boundary condition for the electrode boundary condition in Equation (2-11) was modified as follows to make it non-linear:

$$
\left.\mathbf{i}(\mathbf{x})\right|_{W E}=-\left.\kappa \mathbf{n} \cdot \nabla \Phi(\mathbf{x})\right|_{W E}=\left(\frac{1-j \omega R_{W E} C_{W E}}{R_{W E}}\right)^{\alpha}\left(\Psi-\left.\Phi(\mathbf{x})\right|_{W E}\right)
$$

where $\alpha$ is an exponential constant, $0.5 \leq \alpha \leq 1.0$

The empirical exponent, $\alpha$, was set to a value between 0.5 and 1.0. A value of $\alpha=0.5$ produced an impedance spectra resembling the Gerischer response [26], a non-

\footnotetext{
* Side note: Although the intent of this electrode model is empirical in nature, it does have a form
} similar to an $\alpha$-Lévy process [36], a type of stochastic process with application to physical phenomena such as Brownian motion. 
ideal response observed in a number of solid-state electrochemical systems [27]. Figure 2-6 (b) displays an example of the impedance response of this non-linear electrode model.

\subsubsection{Values of material properties and electrode parameters}

The ionic conductivity, $\kappa$, for the electrolyte pellet material was set to $0.065 \Omega$ $\mathrm{cm}^{-1}$; this is the ionic conductivity of YSZ at $900^{\circ} \mathrm{C}$ [28], i.e. the upper end of the temperature range for the EIS experimental measurements in Chapter 5. The resistance, $R_{W E}$, of the working electrode was set to $0.1 \Omega-\mathrm{cm}^{2}$, typical of an oxygen reduction cathode or hydrogen oxidation anode, although the area-specific-resistance (ASR) measurements for $\mathrm{CO}-\mathrm{CO}_{2}$ exchange presented in Chapter 5 are an order of magnitude

larger. The capacitance, $\mathrm{C}$, was set to $1.0 \mathrm{~F} / \mathrm{cm}^{2}$; this value is at the upper end of typical capacitance values reported on oxygen reduction mixed conductor electrodes [29]. Variations of $\mathrm{R}$ and $\mathrm{C}$ values were used to test the effects of different electrodes, as noted in the text and appropriate figures.

\subsection{Analysis and Computational Methods}

Finite element analysis was performed using COMSOL Multiphysics ${ }^{\mathrm{TM}}$ version 3.3 (COMSOL AB). The PDE, coefficient form was used to implement the governing equation and boundary conditions. Lagrange quadratic elements were utilized to generate the mesh and the stationary nonlinear solver was used to generate the solution [30]. 


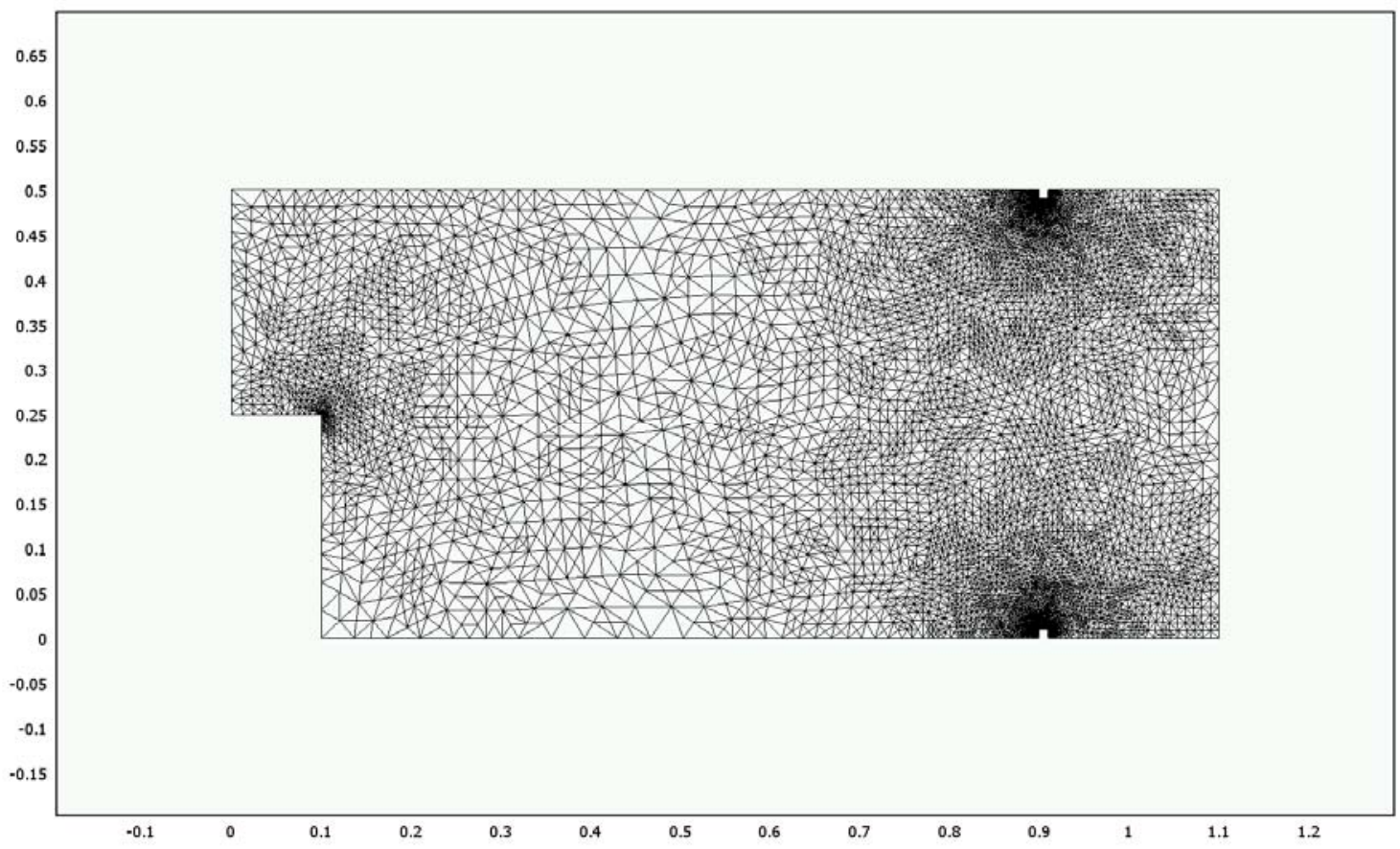

Figure 2-7. A typical mesh of the pellet subdomain. This particular case has 17399 linear elements with 8889 degrees of freedom. The adaptive mesh was turned on and error minimized to $1.6 \times 10^{-4}$. Enhanced meshing at the outer edges of the electrode boundaries is due to the higher potential gradients at a $180^{\circ}$ intersection of electrode and insulator planes under primary current distribution conditions (discussed in text).

\subsubsection{Finite element solution of the potential, $\Phi$, in the electrolyte}

For each frequency point in the simulation, a finite element solution of the potential, $\Phi$, in the pellet electrolyte was obtained. Figure 2-7 displays a typical finite element mesh for a 2D analysis. A typical mesh size was 17,000 elements with 8800 degrees of freedom for the 2D models; the 3D model mesh size was typically 104,000 elements with 151,000 degrees of freedom. The error tolerance for a solution was set to 
$1.0 \times 10^{-6}$. The adaptive mesh refinement algorithm was turned on with the number of mesh elements as the limiting parameter. Several solutions for each new geometry model were run, increasing the number of mesh elements limit until the converged solution was insensitive to mesh size as a further check for sensitivity and convergence of the result. The high gradient areas (i.e. finer mesh) are near the corner of the RE1 reference electrode hole and the outside edges of the WE and CE boundaries. Except for the RE1 corner, both RE1 and RE2 location are, as expected, in low gradient areas. Rounding the RE1 corner in the solid geometry model would have reduced this somewhat, but the mesh refinement was mainly driven by the WE and CE edges.

Figure 2-8 displays a surface plot of the typical numerical solution of the potential in the solid geometry model, with constant potential streamlines superimposed on the color graduated potential gradient. The solution indicates both the RE1 and RE2 locations are in low potential gradient areas, as expected, providing an unambiguous (low varying) potential over the reference electrode area, and as will be seen, minimizes drift of the reference voltage with frequency.

\subsubsection{Checks of the computational solution}

To confirm the sensitivity and convergence of the result of the model, along with debugging errors, two checks were made. The total current density at the WE electrode and $\mathrm{CE}$ electrodes were compared to confirm that continuity was not violated; the total current values of the WE and CE, calculated by numerically integrating over the area of each disk electrode, were typically within $0.1 \%$ of each other. 


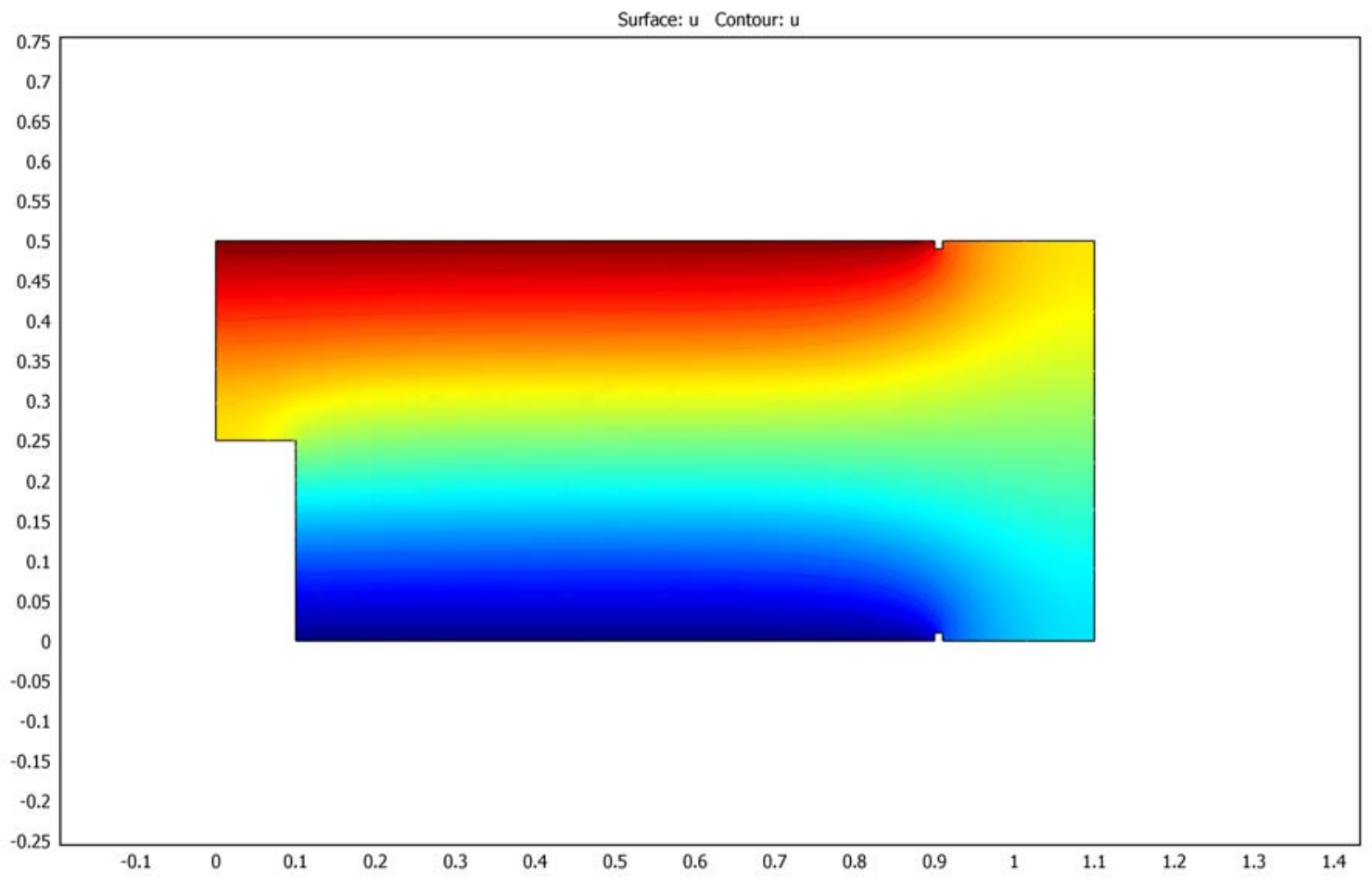

Figure 2-8. A surface plot of a typical finite element analysis (FEA) solution for the pellet geometry. Constant potential streamlines are superimposed on the plot for further definition of the potential profile. Values of key parameters are: angular frequency $\omega=$ $0.1 \mathrm{~s}^{-1}$; both working and counter electrodes have the same response, i.e. $\mathrm{R}_{\mathrm{WE}}=\mathrm{R}_{\mathrm{CE}}=0.1$ $\Omega-\mathrm{cm}^{2}$ and $\mathrm{C}_{\mathrm{WE}}=\mathrm{C}_{\mathrm{CE}}=1.0 \mathrm{~F} / \mathrm{cm}^{2}$; the electrolyte conductivity, $\kappa=0.065 \Omega^{-1}-\mathrm{cm}^{-1}$.

A second check was performed by comparing the average current density computed with the value determined via the electrode potential at the WE boundary, as follows:

$$
i_{w e}=\frac{1-j R_{W E} C_{W E}}{R_{w e}}\left(\Psi_{0}-\left.\Phi_{\text {area-avg }}\right|_{W E}\right)
$$


where $\Psi_{o}=0$

$$
\left.\Phi_{\text {area-avg }}\right|_{W E}=\text { area-averaged electrolyte potential at the WE boundary }
$$

The area-averaged potential, $\Phi_{\text {area-avg }}$, at the WE boundary is computed as:

$$
\left.\Phi_{\text {area-avg }}\right|_{W E}=\frac{1}{A_{\text {electrode }}} \int_{0}^{R} \Phi\left(r, z=H_{p}\right) 2 \pi r d r
$$

where $H_{p}=$ height (or thickness) of the electrolyte pellet

$R=$ radius of the $\mathrm{WE}$

This was compared with the current density computed to calculate the AC impedance, i.e. which is performed by actually integrating the flux of the gradient of the electrolyte potential, $\nabla \Phi$, on the WE boundary:

$$
i_{\text {electrolyte }}=\frac{1}{A_{\text {electrode }}} \int_{0}^{R} \kappa\left[\mathbf{n} \cdot \nabla \Phi\left(r, z=H_{p}\right)\right] 2 \pi r d r
$$

Both the real and imaginary values for current density computed both these ways matched within $0.01 \%$ for the $2 \mathrm{D}$ models and $0.06 \%$ for the $3 \mathrm{D}$ models.

For both the 2D and 3D $18 \mathrm{~mm}$ WE solid geometry models, a tiny "notch" was placed at the electrode/electrolyte surface edge to avoid numerical inconsistencies, which can occur as the current density approaches $\infty$ at the $180^{\circ}$ intersection of an electrode 
plane and an insulator plane [8]. [The "notch" has dimensions of $0.01 \times 0.01 \mathrm{~cm}$, compared to the electrode radius length of $0.9 \mathrm{~cm}$.] Without this modification, it was difficult to resolve continuity to a high degree at the electrode-electrolyte boundary, i.e. current flux into the subdomain at the working electrode equals current flux out at the counter-electrode. The variation in current density is compared with and without this "notch" in Figure 2-9 for a geometry identical to the baseline case geometry, but without the RE1 reference electrode hole. The baseline geometry case (i.e. with the RE1 hole) has been included for comparison.

In Figure 2-9, the current density varies only slightly for "w/o notch" and "with notch" cases, with the integrated current density differing by $1.8 \%$. But the average current density check of the "with notch" case matched within an order of magnitude lower tolerance compared to the "w/o notch" case, and required $10 \%$ fewer mesh elements. The baseline geometry case current density matches these two cases well at the edge of the electrode, but deviates significantly as $r \rightarrow 0$, obviously due to the shielding effect of the RE1 reference electrode hole.

\subsubsection{Other computational details}

In order to perform a complete EIS simulation, the solid geometry models, built in COMSOL Multiphysics, were converted to script files, or M-files. Then, script code, written in MATLAB (MathWorks, version 7.1), was added to repeatedly solve the finite element model as the angular frequency $(\omega)$ was varied, perform post-processing 


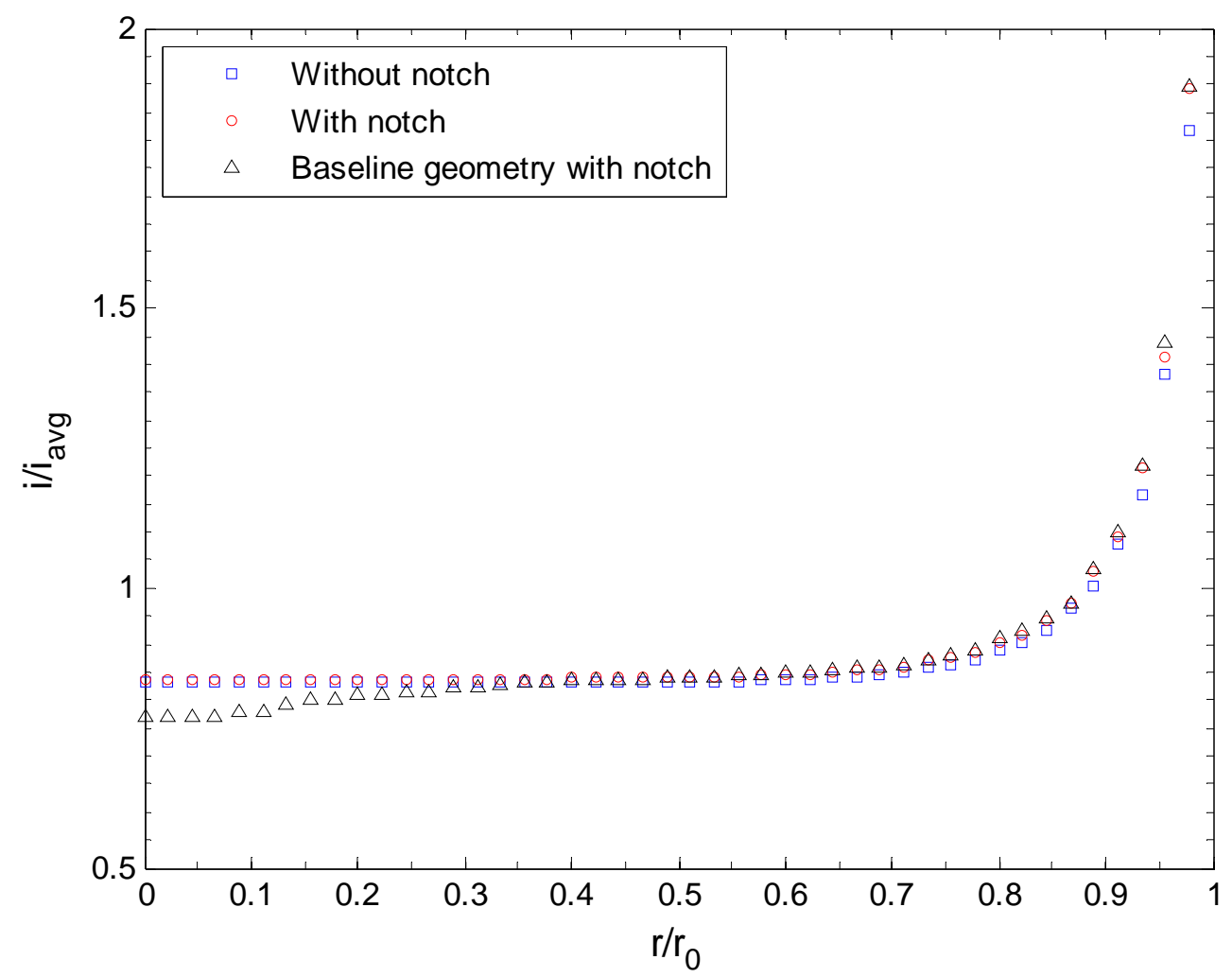

Figure 2-9. Computational results for the current density along the radius of the working electrode (WE) to show the effect of modifying the outer edge of the electrode with a notch (shown earlier in Figure 2-4). The "with notch" and "without notch" cases utilize a cylindrical pellet geometry without the RE1 hole shown in Figure 2-4. Results are for the $\omega=0$ solution.

functions, and generate the plotted results in this chapter. The angular frequency range evaluated for a typical simulation was from $10^{-2}$ to $10^{+4} \mathrm{~s}^{-1}$ with 10 frequency points per decade, with two additional end points, 0.0 and $1 \times 10^{-8}$ such that the spectra would intersect the real axis in a Nyquist plot; this corresponded to a total of 52 individual solutions per simulation. The error for a particular geometry case was evaluated by computing the error in the magnitude of $Z, \Delta|Z|$ error, and the error in the phase angle, 
$\Delta \theta$ error, for each individual simulated computed point, comparing it to the value for the ideal RC electrode, and reporting the single maximum error value for the whole set of simulated points in the given simulation, i.e.

$$
\begin{gathered}
\Delta|Z| \text { error }=\max \left[\frac{|Z|_{\omega_{n}}-\left|Z_{\text {ideal RC }}\right|_{\omega_{n}}}{\left|Z_{\text {ideal RC }}\right|_{\omega=0}}\right] \text { and, } \\
\Delta \theta \text { error }=\max \left[\frac{\theta_{\omega_{n}}-\left(\theta_{\text {ideal RC }}\right)_{\omega_{n}}}{\left(\theta_{\text {iddeal RC }}\right)_{\omega=0}}\right]
\end{gathered}
$$

In most, if not all cases evaluated, the maximum error occurred for the $Z(@ \omega=0)$

point, with $\Delta \theta$ error $\ll \Delta|Z|$ error, typically one or several orders of magnitude lower.

The simulations were performed on an HP Compaq nc8430 personal computer with an Intel $2.0 \mathrm{GHz}$ Core Duo ${ }^{\mathrm{TM}}$ microprocessor and $1.0 \mathrm{~GB}$ of RAM. For a single 2D simulation consisting of 52 frequency points, the computational time was on the order of 2-5 minutes; a single 3D simulation typically required 4-5 hours of computation time.

\subsection{Results and Discussion}

\subsubsection{Impedance simulation for the Baseline geometry}

Figure 2-10 displays the simulation results for the baseline cell geometry with symmetric linear RC electrodes. The simulation indicates the response for three measurements, WE to RE1, WE to RE2, and WE to CE (labeled as full cell). This full 
cell measurement is appropriate if the cell is symmetric, i.e. having an identical WE and CE; these full cell impedance values are divided by 2 to directly compare with the RE1 and RE2 measurements. All three measurement results have the same single semicircular arc as the ideal RC electrode response, but the arcs are larger indicating the simulated impedance for these measuring points is greater than the true electrode impedance. The maximum error for the RE1 electrode position is $5.8 \%$ and the RE2 (or edge) electrode and full cell measurement errors are larger at 15.3\%. Inspecting both Figures 2-10 (a) and (b), it is apparent the maximum error occurs for $|Z|$ at $\omega=0$. From the shape of the impedance arcs for all three measurement points in Figure 2-10 (a), there appears to be little or no distortion in the responses, when compared to the semi-circle arc for the ideal RC electrode response. In Figure 2-10 (b), the shape of the error in $|Z|$

curves for all three measurement points have the same shape as a Bode plot of $|Z|$ for the ideal RC electrode, further confirming these measurement points produce a near linear response. Winkler et al. [15] reported a similar error for their computational analysis ( at $\omega=0$ and $\infty$ ) of the 3-electrode Risø pellet geometry, which utilizes a centered reference electrode hole similar to the RE1 location in our baseline cell geometry. Adler [16] also reported negligible distortion and similar magnitude for errors in $|Z|$ in his computational simulations of a thick electrolyte cylindrical geometry with a centered and core-drilled reference electrode hole. 


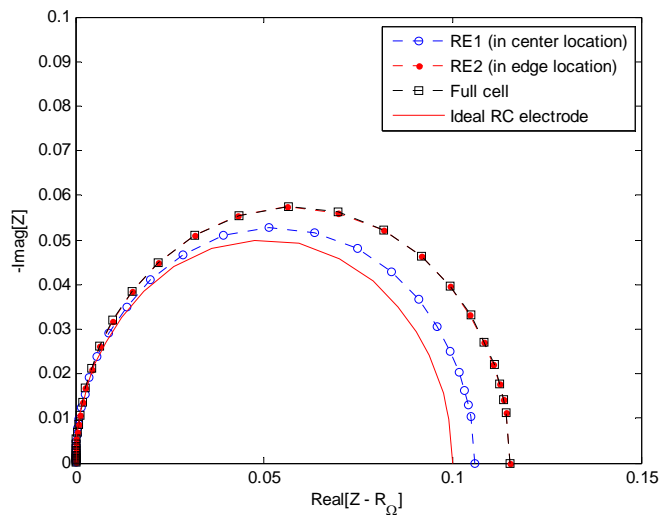

(a)

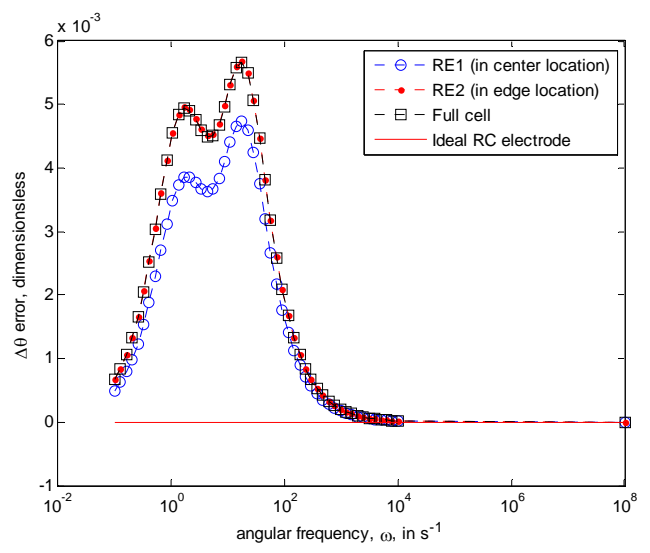

(c)

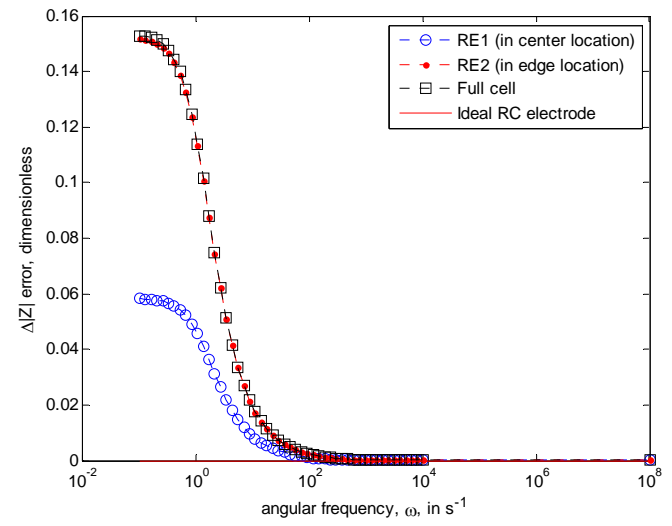

(b)

Figure 2-10. Impedance spectral response simulation of symmetric electrodes for the baseline cell geometry (see Figure 2-1). Ohmic resistance due to the electrolyte has been subtracted from the real impedance responses. The full cell response has been divided by 2 for direct comparison with the ideal working electrode and reference electrode simulations. (a) Nyquist plot, (b) Bode plot of the difference in the magnitude of the electrode impedance, $\Delta|Z|$ error, compared to the ideal RC electrode response, and (c) Bode plot of the difference in the phase angle, $\Delta \theta$ error, compared to the ideal electrode response. 


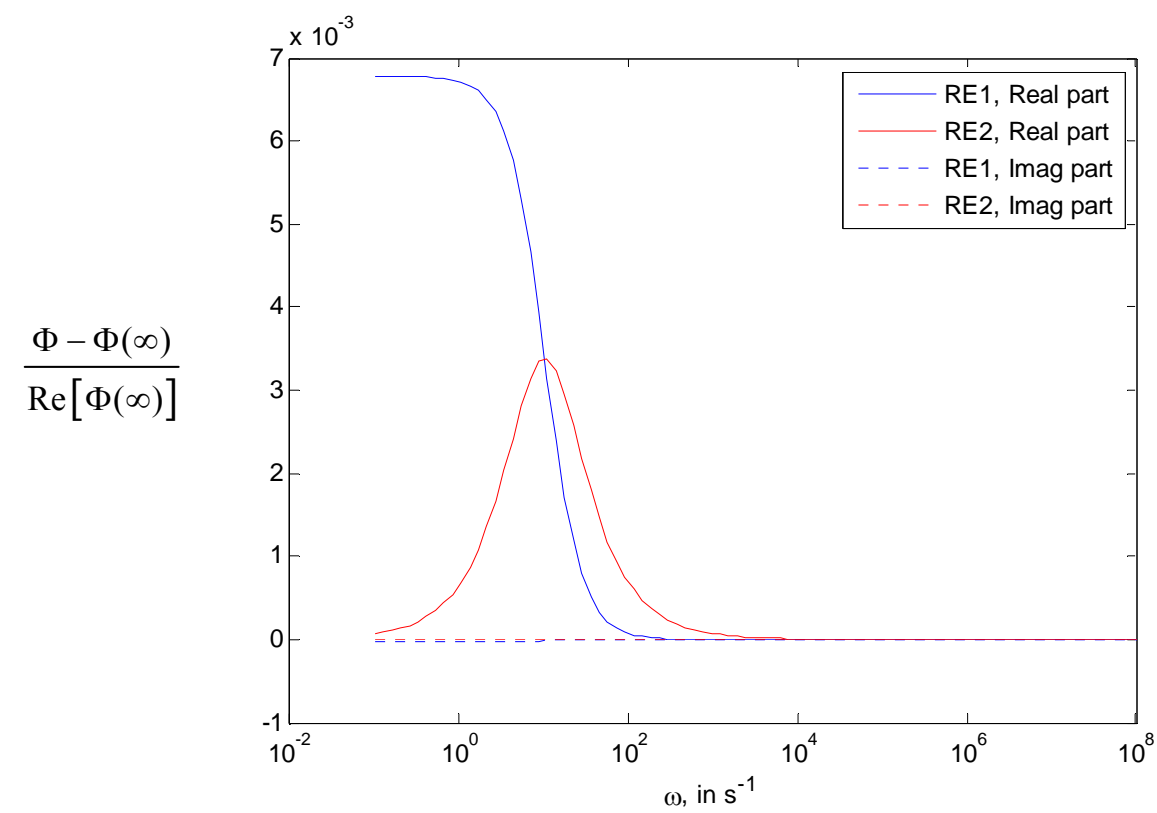

(d)

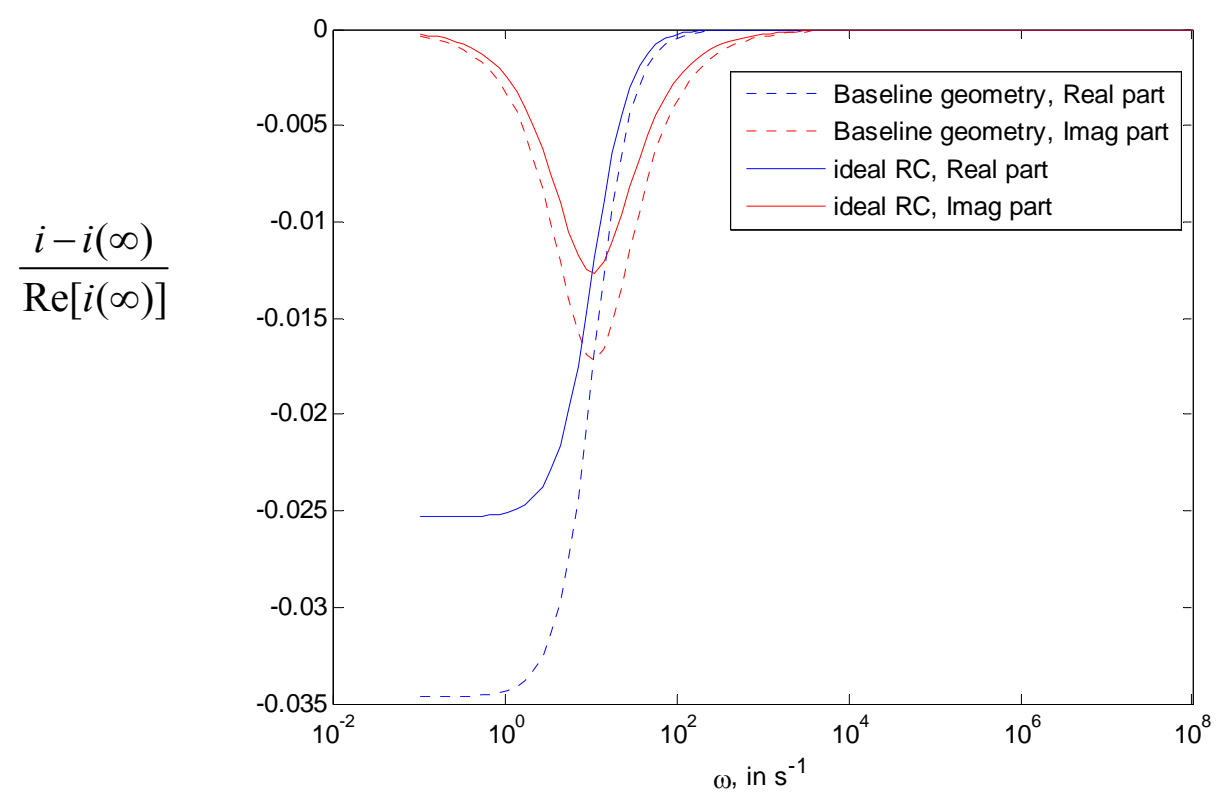

(e)

Figure 2.10 (cont.) (d) Drift of the RE1 and RE2 reference electrode potentials versus frequency. Abscissa is the difference in $\Phi$ divided by the real part of $\Phi$ at high frequency. (e) Corresponding plot of the variation in current density with frequency at the WE. 
Figure 2-10 (c) displays the corresponding errors in phase angle, $\theta$, as a function of frequency. Although the error in $\theta$ appears to be highly non-linear, it is two orders in magnitude less than the $|Z|$ error, and can be considered negligible compared to the $|Z|$ error. The magnitude of the $\theta$ errors $\left(6 \times 10^{-3}\right)$ is about two orders of magnitude larger than the numerical error in the computational solution.

Figure 2-10 (d) displays the drift of the RE1 and RE2 potentials as a function of angular frequency, expressed as a fractional difference in $\Phi$ divided by $\operatorname{Re}(\Phi(0))$. This figure indicates the drift of the reference electrode potentials with frequency is small, and certainly does not account for the total error in Z. Figure 2-10 (e) displays the variation current density with frequency compared to the ideal $\mathrm{RC}$ electrode response. This result shows the current density variation is the larger contributor to the $|Z|$ error for this pellet geometry.

\subsubsection{Impedance simulation for the Baseline case with non-linear electrodes}

Figure 2-11 displays the results for the baseline geometry and symmetric nonlinear electrodes; in this case, the non-linear model in Equation (2-16) is used for both the WE and CE boundary conditions with the same electrode parameter values. Figure 2-11 (a) is Nyquist plot of the impedance response of the RE1, RE2, and full cell measurements. The results are very similar to the linear symmetric electrode case. 


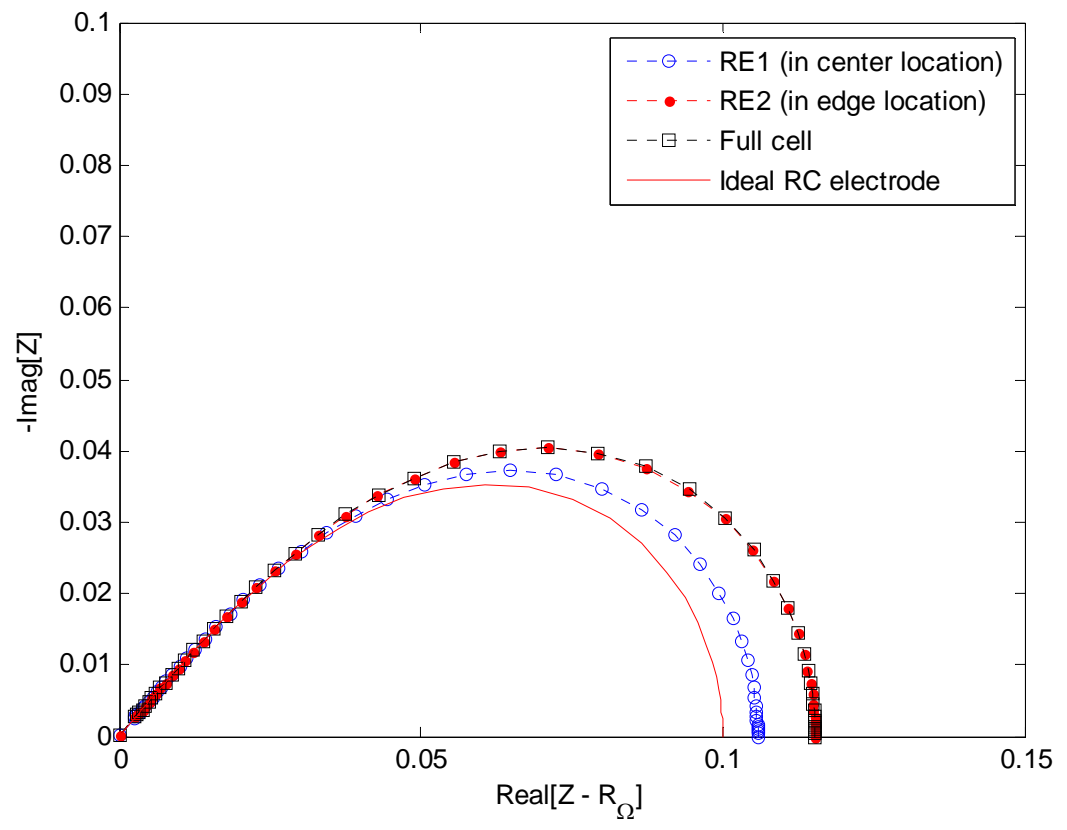

(a)

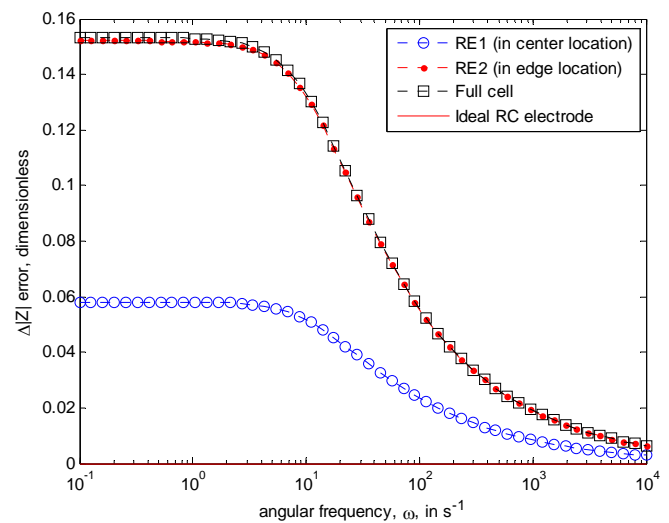

(b)

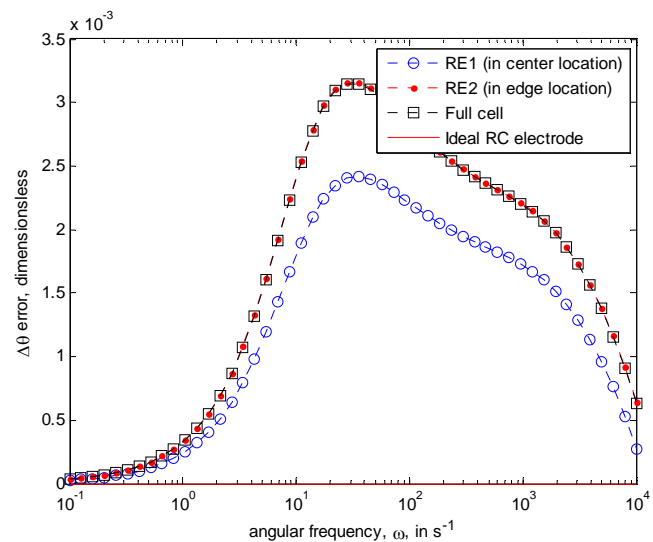

(c)

Figure 2-11. Impedance spectral response simulation of the baseline cell geometry with non-linear but symmetric WE and CE. Electrode parameters are $R_{W E}=R_{C E}=0.01, C_{W E}$ $C_{C E}=5, \alpha_{W E}=\alpha_{C E}=0.50$. Ohmic resistance due to the electrolyte has been subtracted from the real impedance responses. The full cell response has been divided by 2 for direct comparison with the ideal working electrode and reference electrode simulations. (a) Nyquist plot, (b) Bode plot of the difference in the magnitude of the electrode impedance, $\Delta|Z|$ error, compared to the ideal non-linear electrode response, and (c) Bode plot of the difference in the phase angle, $\Delta \theta$ error, compared to the ideal electrode response. 
All three measurements are shifted to the right, i.e. the measured impedance is greater than the "ideal" non-linear electrode, but the shape of the impedance arcs indicates the response is not distorted. The error for the RE1 electrode measurement is $5.8 \%$ and the RE2 and full cell measurements are $15.3 \%$, matching the results from the linear electrode case. The indication is these errors are solely a function of cell geometry, i.e. predictable and independent of the electrode properties and could potentially used as correction factors to correct measurements to the true electrode values. Figures 2-11 (b) and (c) display the Bode plots of the error in $|Z|$ and $\theta$, respectively. As with the linear electrode case, the error in $|Z|$ is the major contributor and the error in the phase angle, $\theta$, can be neglected.

\subsubsection{Impedance simulation for the Baseline case with non-symmetric electrodes}

The previous two analyses employing symmetric electrodes are special cases in EIS experimental work. In most experimental situations and practical applications, the WE and CE will not have identical impedance responses. Even if they are identical in material and microstructure, they may be exposed to different atmospheres (e.g. fuel or air), and their corresponding impedance responses will not be identical due to the different kinetic or mass transfer conditions. As described in section 2.1, a nonsymmetric CE can distort the measurement of the desired WE response. For example, West and Newman [31] performed an analysis on a channel flow cell geometry and showed how the kinetics of a counter-electrode can affect the measurement of the working electrode kinetics for perfectly aligned working and counter-electrodes. 


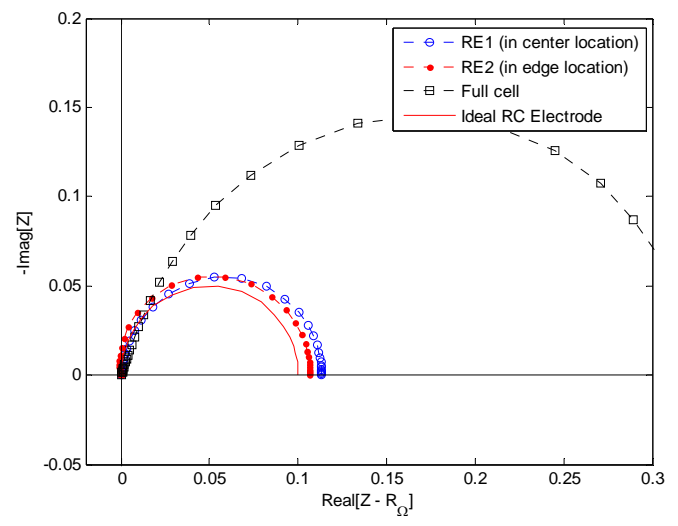

(a)

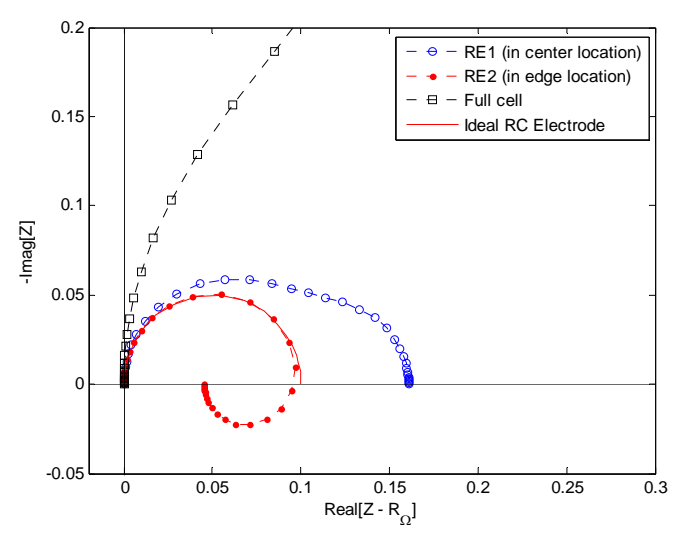

(c)

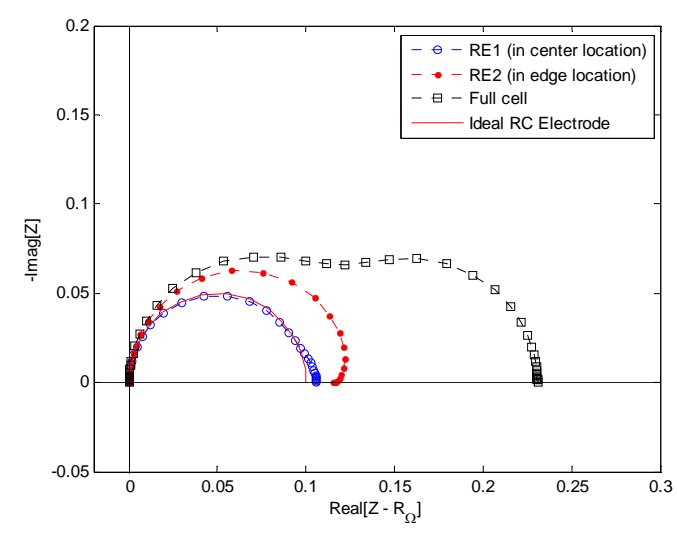

(b)

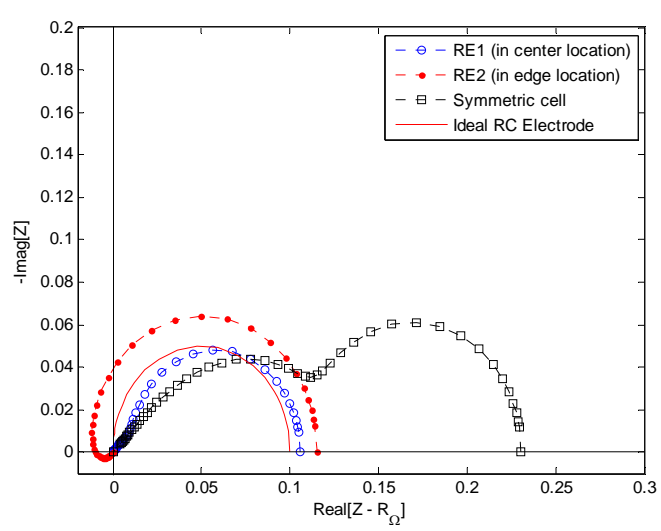

(d)

Figure 2-12. Nyquist plots of the baseline cell geometry with non-symmetric electrodes. Baseline electrode parameters for linear and symmetric WE and CE are $R_{W E}=R_{C E}=0.1$, $C_{W E}=C_{C E}=1.0, \alpha_{W E}=\alpha_{C E}=1.0$.

(a) Linear WE and slightly non-linear CE, i.e. $\alpha_{W E}=1.0, \alpha_{C E}=0.75$.

(b) Linear WE and CE with higher capacitance CE, i.e. $C_{W E}=1.0 C_{C E}=10$.

(c) Linear $\mathrm{WE}$ and $\mathrm{CE}$ with higher resistance $\mathrm{CE}$, i.e. $R_{W E}=0.1, R_{C E}=10$.

(d) Linear WE and highly non-linear CE are $R_{C E}=0.01, C_{C E}=0.5, \alpha_{C E}=0.50$. 
Figure 2-12 contains the results of a set of simulations with a linear WE, and CE's with various parametric settings to assess the effect on the measured impedance response of the WE. For this figure, the full cell response has not been divided by 2 because of the non-symmetric electrode assumptions for this set of analyses. Figure 2-12 (a) displays the WE impedance response for a slightly non-linear $\mathrm{CE}\left(\alpha_{C E}=0.75\right)$; for this minor change the response of the WE is linear with the error similar in magnitude to symmetric electrodes for the RE1 and RE2 locations given in Figure 2-10. Figure 2-12 (b) shows the responses of the WE with a $\mathrm{CE}$ that has an order of magnitude larger capacitance, i.e. $C_{C E}=10$. The full cell response has 2 distinct arcs, indicating the WE and CE difference in time constants due to the large difference in capacitance. The RE1 location measurement matches the ideal RC electrode response quite well, but deviates to a small degree at the low frequencies. The RE2 edge location has a large drift that is linear at the high and medium frequencies, but has a significant distortion at the low frequencies.

Figure 2-12 (c) contains the responses of the WE with an order of magnitude larger resistance, i.e. $R_{C E}=10$. Both the RE1 and RE2 location responses have significant non-linear distortions. The RE1 response appears to contain a secondary arc and deviates by more than $50 \%$ error in $|Z|$ as $\omega \rightarrow 0$. The RE1 location arc closely matches the ideal $\mathrm{RC}$ arc but contains a large inductance arc (i.e. positive imaginary response) at the low frequencies, which results in large errors in $\theta$. Finally, Figure 2-12 (d) contains the results for a highly non-linear CE and the R and C parameter settings different than the WE, i.e. $R_{C E}=0.01, C_{C E}=0.5, \alpha_{C E}=0.50$. The full cell response has two distinct arcs that distinguish the non-linear $\mathrm{CE}$ and linear WE responses, again due to 
the difference in $\mathrm{R}$ and $\mathrm{C}$ values between the two electrodes. The $\mathrm{RE} 1$ location response has a small high frequency arc closely matching the high frequency portion of the CE response, and a large secondary semi-circular arc, closely matching the ideal WE but shifted to the right. The RE2 location response has a small high frequency induction loop; the mid and low frequencies consist of a single semi-circular arc, but it is shifted slightly to the left past the $\operatorname{Re}(Z)=0$ axis and into the $-\operatorname{Re}(Z)-\operatorname{Im}(Z)$ quadrant of the Nyquist plot. In these 4 cases, the cause of the distortion is due to a large increase in magnitude of the drift in RE1 and RE2 reference voltages with frequency. Also, the working electrode current density also varies with frequency in a non-linear fashion compared to the current density of the ideal RC WE.

\subsubsection{Discussion of low and high frequency inductive loop artifacts in the literature}

The impedance response distortions observed in the set of simulations shown in Figure 2-12 warrant further discussion, as similar artifacts have been reported in experimental impedance measurements, but not always traced to cell geometry as the cause. For instance, both low and high frequency inductive artifacts have been reported in the impedance literature. Boukamp [2] observed a low frequency inductive loop on $\mathrm{BiCuVO}_{\mathrm{x}}$ working electrode and Au counter-electrode on a thick cylindrical YSZ electrolyte with a Fe implanted layer in the YSZ. Both the working electrode and counter-electrodes are annular with the Au reference electrode mounted in the center of the annulus of the working electrode surface. He attributed this low frequency inductive loop observation due to "crosstalk" between the working and reference electrodes due to surface diffusion of mobile charge oxygen species along the surface of the Fe implanted 
YSZ. Fafilek [32] also observed a low frequency loop for 4 piont measurements on a single crystal mixed bismuth vanadium oxide, $\mathrm{Bi}_{4} \mathrm{Cu}_{2 \mathrm{x}} \mathrm{V}_{2(1-\mathrm{x})} \mathrm{O}_{(11-3 \mathrm{x})}$, (or BICUVOX) electrolyte with Pt or Au point electrodes. He attributes this to electronic crosstalk between different electrodes due to small electronic conduction contribution of this mixed conductor electrolyte. Reed et. al. [33] laminated a reference electrode consisting of a thin strip Pt layer laminated between layers of a tape-caste YSZ electrolyte for a conventional Ni-YSZ/YSZ/LSM cell and observed a low frequency inductance loop for the LSM cathode in their impedance spectrum. McIntosh et al. [34] observed a high frequency inductance loop for an LSM-YSZ WE and Pt CE on a thick pellet cell with RE2 type edge reference electrode; they observed this only under higher current densities and also noted similar behavior for a pellet cell with a drilled hole RE1 type reference electrode. Finally, Gabrielli [35] reviews a number of examples of both high (and low) inductive loop artifacts in the aqueous electrochemistry literature, most notably in corrosion research.

Most, but not all, of these examples provide a brief qualitative discussion of the effect of cell geometry as a possible explanation for their observed phenomena, or experimental evidence to discount it, but none provide a detailed computational analysis of their geometry cell to clarify this possibility.

\subsubsection{Case 1 analysis}

In this section, a computational analysis is presented for a slightly simpler, more ideal, cylindrical geometry cell, which will be referred to as the "Case 1" geometry. The Case 1 geometry cell, shown in Table 2-1, is identical in dimensions to the baseline 
geometry cell, but the WE and CE electrodes completely cover the two faces of the pellet, i.e. the electrodes have a diameter of $1.1 \mathrm{~cm}$. This allows for a more uniform current density distribution in the solid electrolyte, as the electrode edge is perpendicular to the pellet circumference edge, i.e. an insulating plane [8].

The maximum error in $|Z|$ for the RE1 electrode location is $-2.3 \%$, the negative value indicating the semi-circular arc underestimates the impedance of the ideal $\mathrm{RC}$ electrode. This error is in the less than 5\% range reported by Winkler et al. [15] for their much thicker Risø pellet, and indicates that a thinner electrolyte can achieve low measurement errors if the WE and CE completely cover the pellet faces in a cylindrical cell geometry, and the reference electrode hole is not too close to either the working or counter-electrodes. The edge and symmetric cell error for this cell geometry is even lower at $-0.5 \%$, further indicating a more uniform current distribution in the electrolyte, compared to the baseline geometry.

\subsubsection{Impedance simulation for the Case 1 geometry with non-symmetric electrodes}

Figure 2-13 displays the results of a simulation of the Case 1 geometry with nonsymmetric electrodes. The non-linear model electrode boundary condition for the CE was used with the identical parameters for the simulation in Figure 2-10 (d) for the baseline geometry. The most obvious result is that the Case 1 geometry completely eliminates the artifacts observed for the baseline geometry RE1 and RE2 responses in

Figure 2-12 (d). The maximum error in $|Z|$ is also very low, $-2.1 \%$ and $-0.4 \%$ for the RE1 and RE2 impedance responses respectively. 
Case 1:

$\frac{H_{p}}{D_{p}}$ ratio $=0.23$

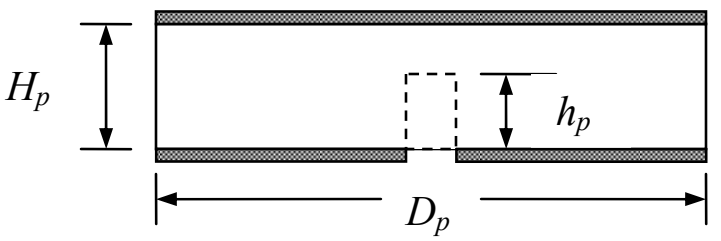

Parameters varied: hole depth, $h_{p}$, nonsymmetric electrodes

Case 2:

$\frac{H_{p}}{D_{p}}$ ratio $=0.45$

Parameter varied: hole depth, $h_{p}$, hole diameter, $d_{p}$

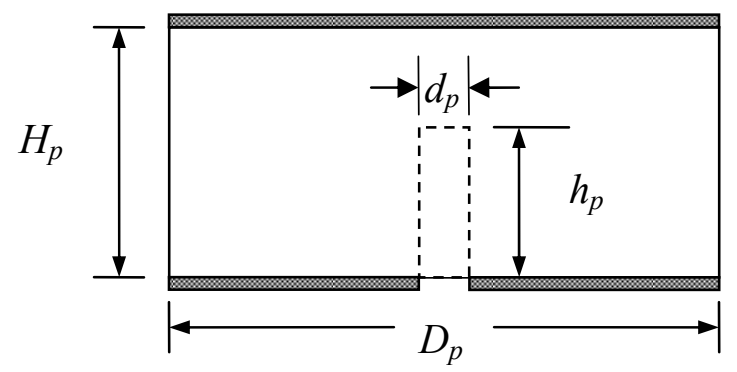

Case 3:

$\frac{H_{p}}{D_{p}}$ ratio $=0.91$

Parameter varied: hole depth, $h_{p}$

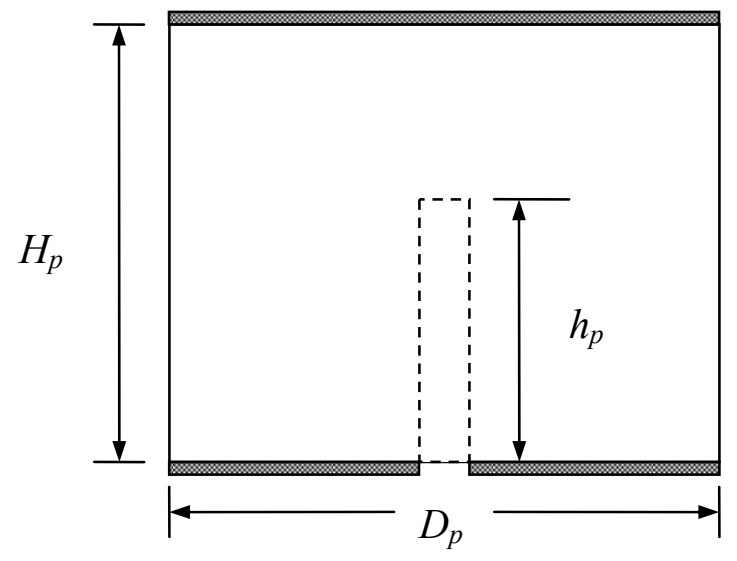

Baseline case:

$\frac{H_{p}}{D_{p}}$ ratio $=0.23$

$18 \mathrm{~mm}$ diameter electrodes

Parameter varied: hole depth, $h_{p}$

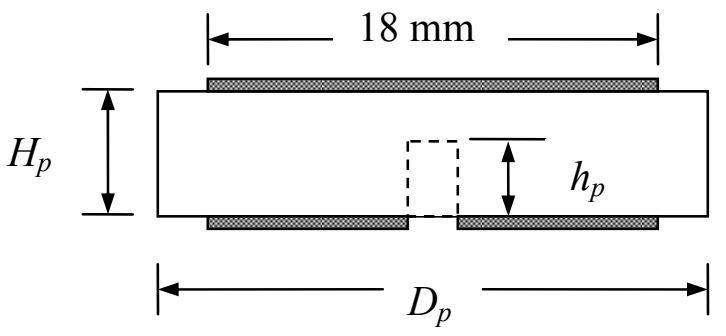

Table 2-1. Description of pellet geometries for the 2D parametric analysis cases. 


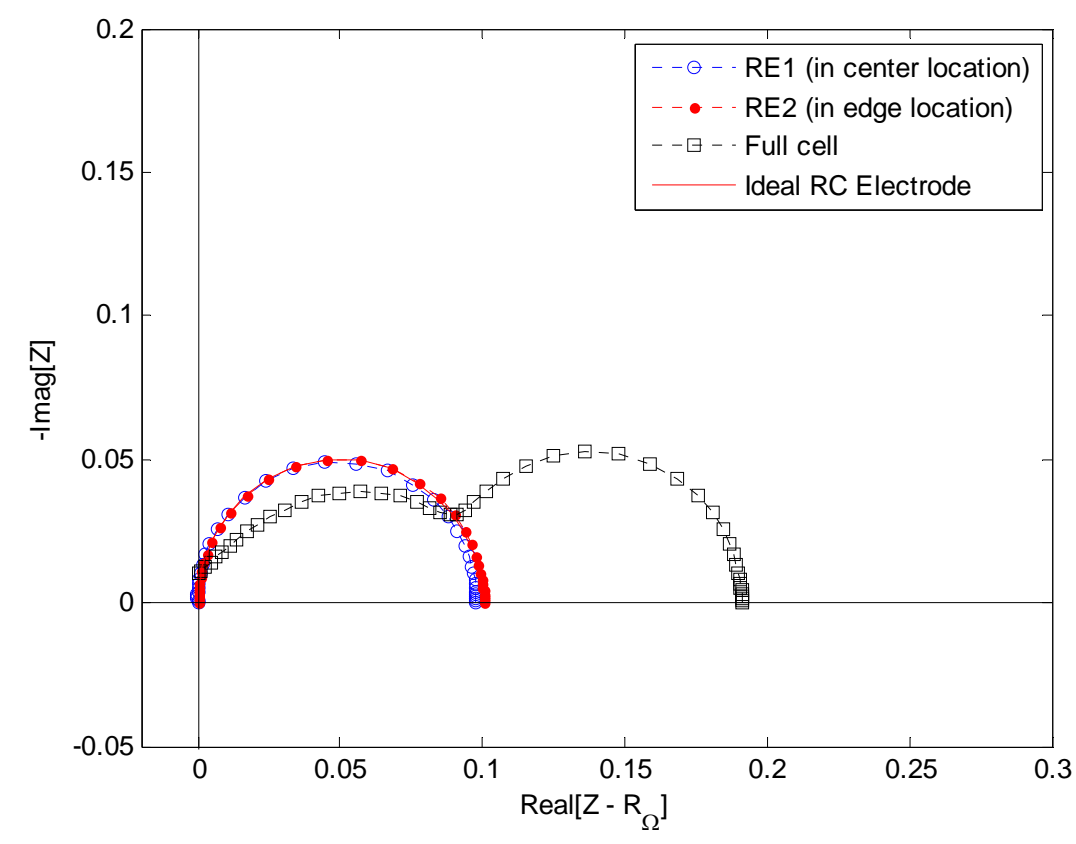

Figure 2-13. Nyquist plot of the Case 1 cell geometry with non-symmetric electrodes. WE and CE parameters have the same values as the case in Figure 2-10 (d) for direct comparison, i.e. $R_{W E}=0.1, R_{C E}=0.01, C_{W E}=1.0, C_{C E}=0.5, \alpha_{W E}=1.0, \alpha_{C E}=0.50$.

Although the Case 1 cell geometry provides a marked improvement over the baseline geometry in providing an accurate impedance response, note that it is less flexible for experimental use; e.g. with the electrodes completely covering the faces, there is no surface provided for sealing to isolate WE and CE in two gas atmosphere experiments. 


\subsubsection{Parametric analysis of the cylindrical pellet geometry}

In this section, a parametric analysis of the cylindrical pellet geometry is performed, varying the key dimensions of the pellet, pellet height or thickness $\left(H_{p}\right)$, pellet diameter $\left(D_{p}\right)$, reference hole depth or height $\left(h_{p}\right)$, and reference hole diameter, $\left(d_{p}\right)$. Table 2-1 provides a list of the 4 geometries investigated to determine the impact of these dimensions on impedance measurement error. Figure 2-14 (a) displays the maximum error in $|Z|$ with reference electrode hole depth (expressed as the ratio, $\frac{h_{p}}{H_{p}}$ ), for all 4 geometries. All the cases have the smallest error in $|Z|$ when the hole depth corresponds to the midpoint in the pellet. The errors in $|Z|$ increase as the hole depth approaches the pellet thickness, due to the "shielding" effect [8]; as the RE1 hole location approaches the WE, the current density is lowered near the center of the electrode due to the reference electrode's close proximity (previously shown in Figure 2-9). The error also increases as the hole depth is decreased, and the RE1 location approaches the CE; this is mainly due to increasing the electrolyte potential gradient, causing larger drifts in the RE1 potential (i.e. area-averaged across the hole diameter). For the larger pellet thicknesses, i.e. Cases 2 and 3, the error decreases with pellet thickness; for Case $3\left(\frac{H_{p}}{D_{p}}=0.91\right)$, the error is insensitive to increasing hole depth up to $\frac{h_{p}}{H_{p}}=0.9$. For the baseline geometry, the errors are the largest across the entire range of hole depths, as expected due to the error induced by the non-uniform current density at the WE (discussed earlier) and the error induced by the hole depth being additive. 


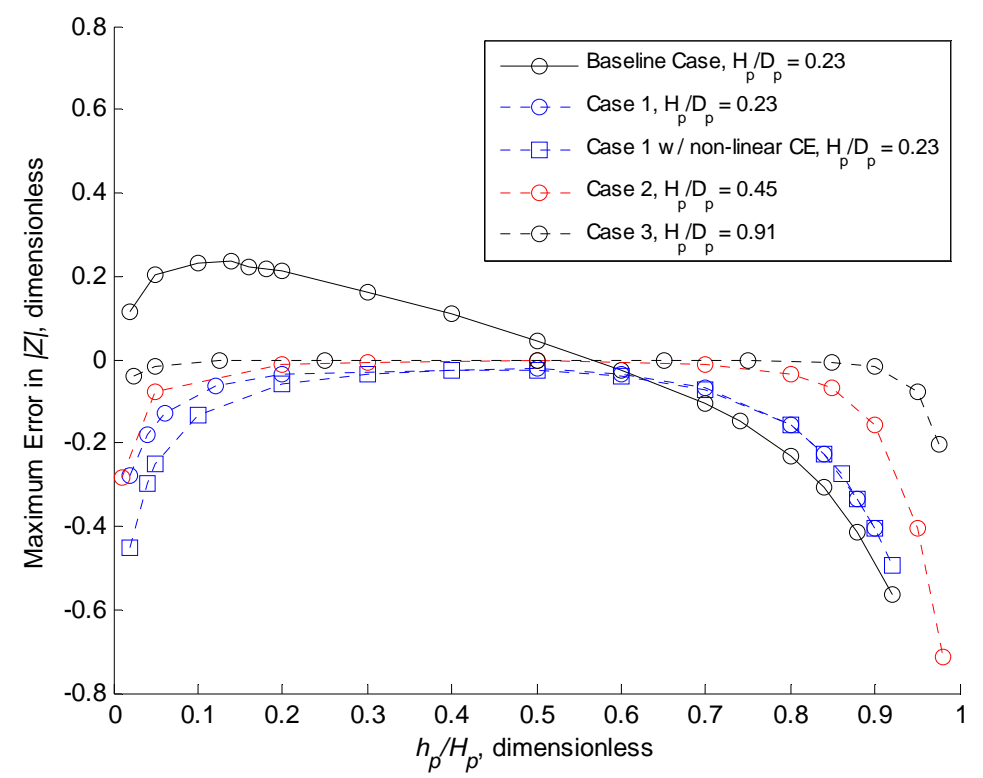

(a)

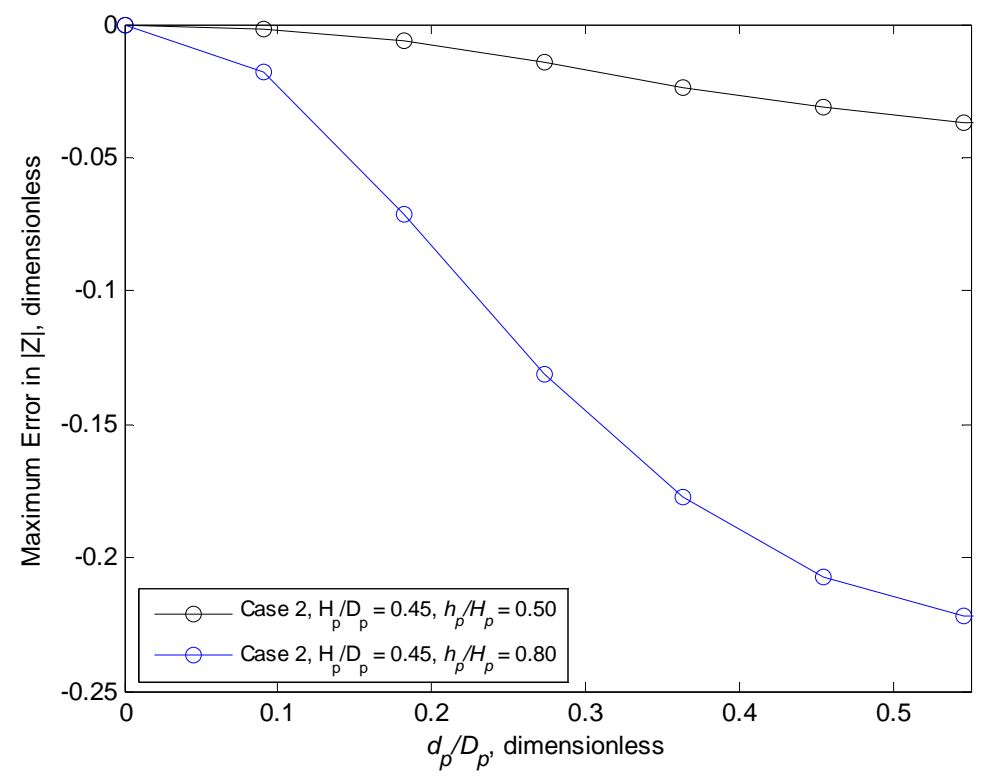

(b)

Figure 2-14. Maximum error in $|Z|$ results for the parametric analyses of the cylindrical pellet geometry. Each point is an individual simulation. (a) Parametric analyses of the reference electrode hole depth for the baseline case, Case 1, Case 2 and Case 3 cylindrical pellet geometries. (b) Variation in the radius of the reference electrode hole for the Case 2 cylindrical pellet geometry. 
Finally, an additional Case 1 analysis with a non-symmetric non-linear CE was included, and indicates there is little change in the error from the Case 1 symmetric electrode analysis. These results show that a pellet thickness beyond a ratio of $\frac{H_{p}}{D_{p}}=0.45$, does not significantly reduce impedance error, and drilling the RE1 hole greater than $1 / 2$ the pellet height does not decrease the impedance error, but rather, can increase the error if is positioned too close to the WE.

Note that the errors for the RE2 (edge) and full cell measurements were also analyzed, but remained constant over the hole depth range(s) analyzed for all 4 of the geometries, and were not included here.

Figure 2-14 (b) shows the results of varying the diameter of the RE1 hole for the Case 2 geometry. As expected, the maximum error in $|Z|$ increases with the RE1 hole diameter due to increased distortion in the current density distribution, and higher gradients in $\Phi$, and this error does increase for the higher depth hole. But surprisingly, the error is small (less than $8 \%$ ) for ratios of $\frac{d_{p}}{D_{p}}$ up to 0.2 . For most practical experiment cells with pellet diameters of $1.0 \mathrm{~cm}$ or greater, this corresponds to an RE1 hole diameter of $2 \mathrm{~mm}$, achievable with conventional tool drill bits or other fabrication techniques. 


\subsubsection{Analysis of misaligned electrodes in the Baseline cell geometry}

In the analyses on the baseline geometry discussed to this point, the WE and CE electrodes are assumed to be perfectly aligned, i.e. centered on each pellet face. A potential additional source of error for the baseline geometry cell is misaligned electrodes. Several researchers [16,19] have performed computational analyses on the effects of misaligned electrodes on thin electrolyte cell geometries, but the implied assumption is that measurements on a thick electrolyte cell geometry are not as susceptible to errors due to misaligned electrodes. In this section, results are presented for several simulations performed to determine the impedance error induced by misaligned electrodes for the baseline pellet cell geometry.

Figure 2-15 (a) defines the WE offset parameter that was varied to assess misalignment error. Because of this offset, the geometry model is no longer axisymmetric, so a 3D finite element model was built using the minimum symmetry condition, a 1/2 pellet, as shown in Figure 2-15 (b). Two simulations ${ }^{\S}$ were performed with the WE offsets, $\Delta l$, of $0.10 \mathrm{~cm}$ and $0.17 \mathrm{~cm}$. The results of these simulations are summarized in Table 2-2, where the WE offset is expressed as a dimensionless

parameter, $\frac{\Delta l}{D_{p}}$. The maximum error in $|Z|$ is in addition to the error due to the baseline cell geometry with aligned electrodes.

\footnotetext{
${ }^{\S}$ A perfectly aligned electrode, i.e. $\Delta l=0.0$, simulation was also performed to confirm the $3 \mathrm{D}$
} model result matched the 2D axisymmetric model result. 


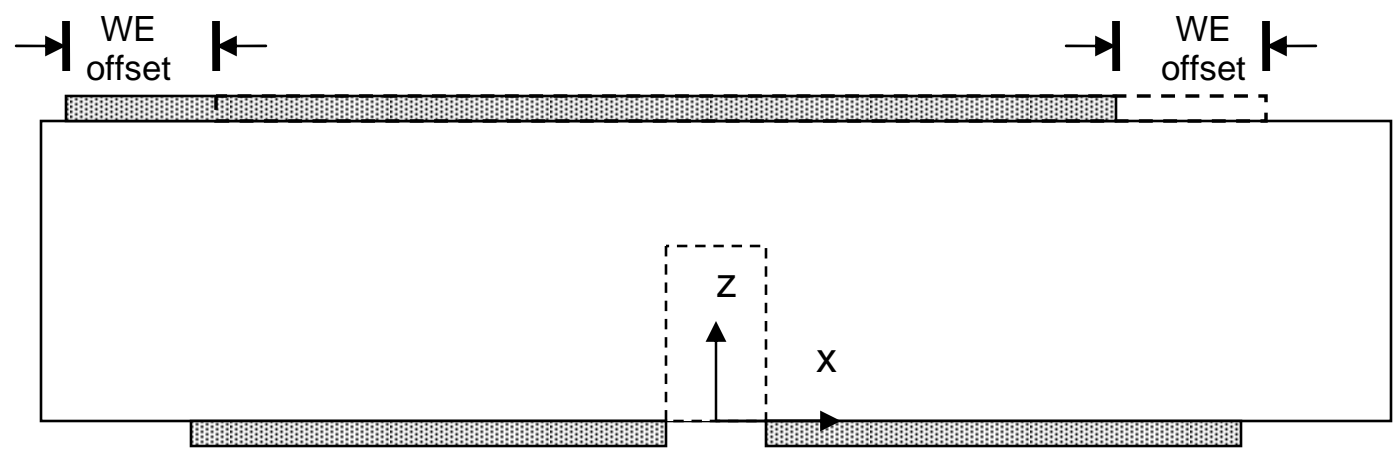

(a)

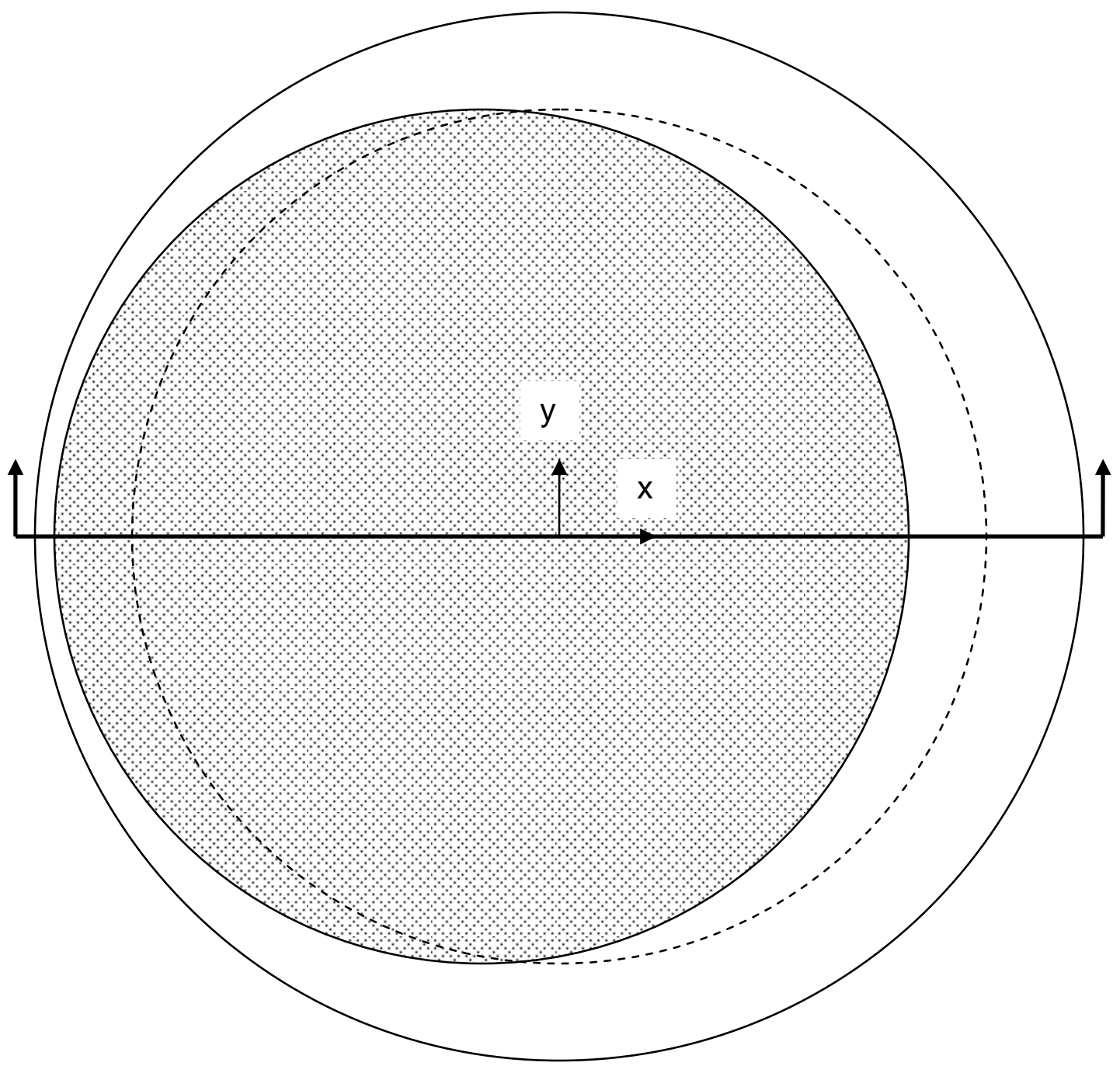

(b)

Figure 2-15. 3D model geometry of pellet cell for working electrode (WE) offset analysis. Computational domain consists of $1 / 2$ of the pellet, i.e. the minimum symmetry condition, as the axisymmetry for the $2 \mathrm{D}$ analysis no longer applies. 


\begin{tabular}{|l|l|l|}
\hline & \multicolumn{2}{|l|}{ WE offset, $\frac{\Delta l}{D_{p}}$, dimensionless } \\
\hline & $\mathbf{0 . 0 6}$ & $\mathbf{0 . 0 9}$ \\
\hline RE1 (hole position) & 0.020 & 0.038 \\
\hline RE2 (edge position) & 0.006 & 0.056 \\
\hline Full cell & 0.017 & 0.012 \\
\hline
\end{tabular}

Table 2-2. Maximum error in $|Z|$ due to misaligned electrodes. The error stated is in addition to the error in the baseline cell geometry with perfectly aligned electrodes.

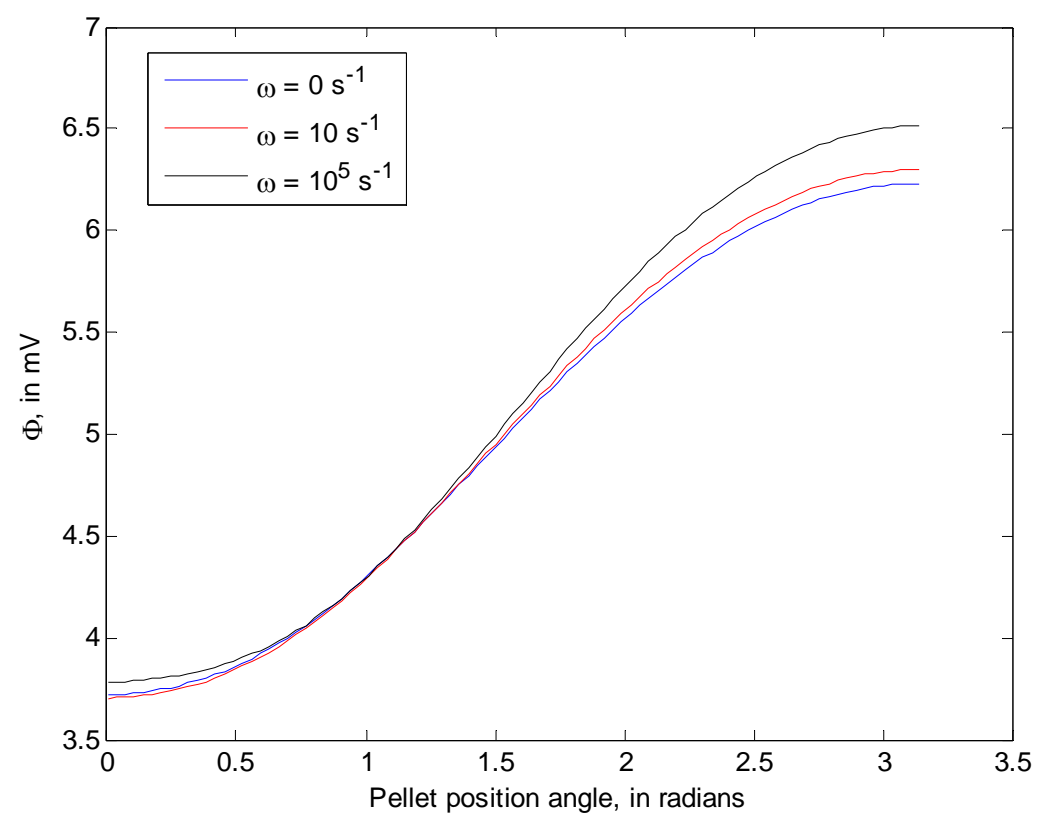

Figure 2-16. Variation in the edge reference electrode (RE2) potential over the circumference of the $1 / 2$ pellet $3 \mathrm{D}$ geometry. WE offset is $0.17 \mathrm{~cm}$. 
The Table 2-2 results indicate that this error due to misalignment for the full cell measurement, at $1.2-1.7 \%$, is minimal, and for the RE1 measurement, at $2.0-3.8 \%$, is slightly less in magnitude than the error for the perfectly aligned geometry. For the RE2 measurement, the error, at $0.6 \%$ to $5.6 \%$ is similar to the other two measurements, but it should be noted the variation in the RE2 potential with position on the circumference of the pellet is significant. Figure 2-16 displays a plot of this variation over the circumference of the $1 / 2$ pellet domain, expressed as the pellet position angle [equal to zero at the $-x$ axis in the $x y$ plane shown in Figure 2-15 (b)]. The RE1 potential varies as much as $30 \%$ from the averaged RE2 potential value used to compute the impedance and resulting error in Table 2-2.

Figure 2-17 displays a plot of the error in the phase angle, $\theta$, for the $0.17 \mathrm{~mm}$ offset simulation, and shows this error has increased substantially from the perfectly aligned electrode case, and is on the order of the error in $|Z|$.

Finally, it should be noted that the simulations of misaligned electrodes reported here should be considered extreme; frankly, this magnitude in misalignment would be easily detectable by eye during fabrication, without any alignment aides. Tolerances for alignment using the standard SOFC electrode fabrication methods, such as screenprinting, are in the range of $125 \mu \mathrm{m}$ [16], much smaller than our offset assumptions. But researchers utilizing thick pellet cells for more fundamental work may utilize electrode fabrication methods (sputtering, painting, air-brushing) that may or may not meet the tolerances of state-of-the-art techniques. 


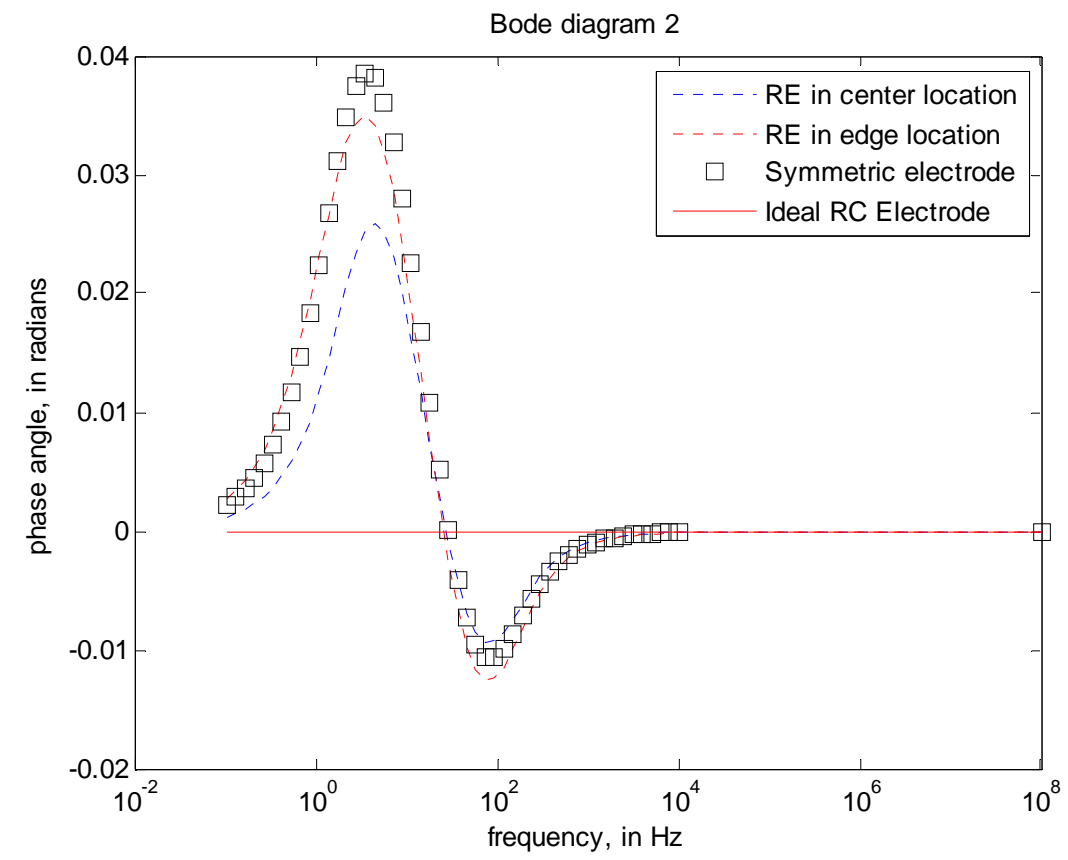

Figure 2-17. Bode plot of the $\Delta \theta$ error for the $0.17 \mathrm{~mm}$ offset case. The phase angle error has increased compared to the perfectly aligned electrode cases, and is on the same order as the $\Delta|Z|$ error.

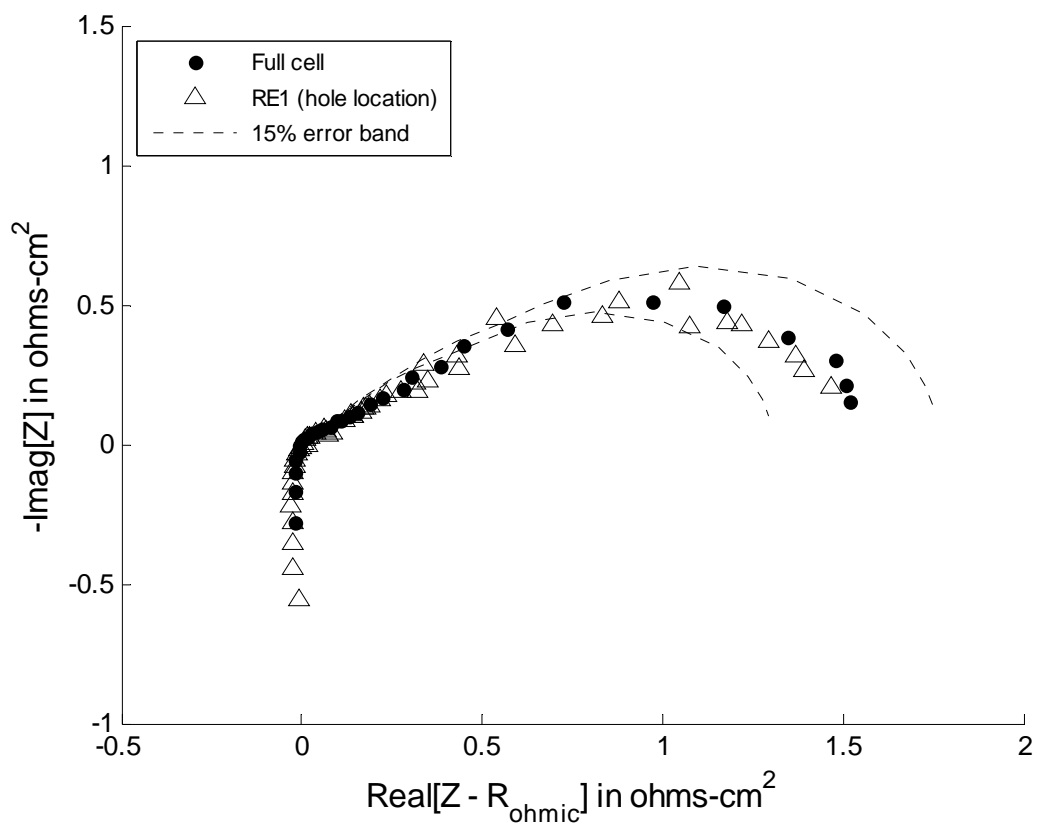

Figure 2-18. Experimental comparison of RE1 and full cell measurements. Impedance measurements taken on a GDC/YSZ/GDC cell with the baseline cell geometry. 


\subsubsection{Comparison of the Baseline cell simulation with experimental results}

Figure 2-18 displays two impedance spectra taken on a GDC/YSZ/GDC baseline geometry cell in a $\mathrm{CO}-\mathrm{CO}_{2}$ atmosphere at $800^{\circ} \mathrm{C}$. Further experiment details can be found in Chapter 5. One of the spectra is a 3 electrode measurement using the RE1 reference electrode location, and the second spectrum is a full cell measurement. Both measurements were made with a zero-bias voltage. $\mathrm{A} \pm 15 \%$ error band was added by fitting the full cell measurement to the $\mathrm{CO}-\mathrm{CO}_{2}$ exchange model in Chapter 4 and adjusting the impedance values by $\pm 15 \%$ to account for the estimated error in $\mathrm{Z}$ calculated for the full cell measurement in section 2.4.1. The results in Figure 2-18 show that these two measurements due lie within the estimated error band due to cell geometry, indicating this cell geometry measure impedance within the estimated error band. But, these results do not support the use of the computational analysis results as a set of correction factors for impedance measurement. In other words, the computational result for this particular case, predicted the full cell measurement should be $\sim 10 \%$ greater than the RE1 measurement (and $\sim 15 \%$ greater than the "true" electrode impedance spectrum). It should be noted that the scatter in the RE1 measurement is larger, possibly due to poor contact of the Pt bead electrode at the RE1 hole location which may be attributed to the cell not being spring-loaded, or instability of the RE1 (Pt) electrode in a $\mathrm{CO} / \mathrm{CO}_{2}$ atmosphere (i.e. as opposed to Pt in air typically utilized for reference electrodes in SOFC work). Similar scatter was observed in other cells tested, and as a result, only for the impedance data from full cell measurements are presented in Chapter 5. 


\subsection{Conclusions}

In this chapter, a detailed set of computational analyses is presented that quantifies the induced impedance error, due to primary current distribution considerations, in the cylindrical thick pellet geometry. The geometry of the electrochemical cell (i.e. the baseline pellet geometry) used for the experimental portion of this work. In addition, a set of parametric analyses is presented to assess a number of ways to reduce measurement errors in simple cylindrical pellet geometries.

For the baseline cell geometry with symmetric electrodes, the maximum error is $6 \%$ for a centered hole reference electrode (RE1) and 15\% maximum error for a circumference mounted reference electrode (RE2) and a symmetric full-cell measurement. For a non-linear electrode response, the error values are similar, indicating the geometry error is reproducible, and not a function of the electrode response. These errors displace the electrode spectra in a linear manner from the true electrode response, with little or no distortion in the shape of the impedance arc.

For the baseline geometry with non-symmetric electrodes, the impedance response for the working electrode does become distorted due to crosstalk from the counter-electrode. In particular, both the RE1 and RE2 reference voltages drift with frequency in a non-linear fashion, along with the current density of the working electrode. These non-linear artifacts in the response, e.g. high or low frequency inductance loops, could be misinterpreted as alternative kinetic or mass transfer phenomena. For the same pellet cylindrical geometry, but both WE and CE completely 
covering the pellet faces, these artifacts due to non-symmetric electrodes are eliminated, and the WE impedance arc has little or no distortion in the shape.

In order to more fully investigate this simple cylindrical pellet geometry, a set of parametric analyses was performed, varying pellet height, reference electrode hole depth, and reference electrode hole depth. The optimum reference electrode hole depth is midway between the working and counter-electrodes, and a thicker pellet allows a larger range of hole depths with minimal errors. In particular, a pellet with a height-to-diameter ratio of 0.9 allows one to place the reference electrode within $10 \%$ of the pellet height close to the working electrode with less than $5 \%$ error. The reference hole diameter can be as large as $10 \%$ of the pellet diameter with minimal error, i.e. a $1 \mathrm{~mm}$ diameter hole for a $1 \mathrm{~cm}$ diameter pellet, certainly achievable with a conventional drill bit.

The final analysis performed on the baseline geometry was an assessment of measurement error due to misaligned electrodes. For this analysis, 3D models of a $1 / 2$ cylindrical pellet slice were modeled with two different offsets of the working electrode. The results show a slight additional increase in error $(<6 \%)$ for the RE1 and RE2 reference electrode positions, and $<2 \%$ increase in error for full (or symmetric) cell measurements. But it should be noted that phase angle errors also increase to $3-4 \%$, on the order of the $|Z|$ error.

These results show that a very thick pellet electrolyte pellet is not necessarily needed to properly isolate the working electrode from the counter-electrode. But, the 
error for a thick electrolyte pellet is increased if the electrodes do not completely cover the pellet faces, and this is mainly due to the non-uniform current density caused by the electrode edge. Using this design approach also eliminates the additional, but small, error due to misaligned electrodes. Unfortunately, this full electrode face design does not allow for a surface to properly seal the working electrode from the counter-electrode for separate gas atmosphere experimental studies, but this can be solved by selection of a slightly more complex cylindrical geometry such as the Risø pellet design.

It is anticipated for the foreseeable future, solid state electrochemical researchers will continue to utilize thick pellet geometries in "button" type cells to perform, for example, fundamental studies, initial electrochemical measurements for characterization of new electrode materials, or testing variations in electrode fabrication methods. The ease of fabricating small electrode areas (i.e. $1.0-2.0 \mathrm{~cm}^{2}$ ) on thick pellet cells using mature fabrication techniques such as screen-printing or spray painting, allow researchers to quickly and comparatively assess the viability of promising new electrode materials or optimize the formulation and microstructure design of state-of-the-art electrodes.

In this chapter, we have chosen several simple geometries to analyze, whereas a wide variety of thick pellet geometries are used depending on the lab where the work is done. With the easy access to modern easy-to-use finite element software that can be run on a typical configured desktop personal computer or laptop, it is relatively straightforward for today's researcher to evaluate and optimize the particular geometry cell he chooses for his research. 


\section{References}

[1] Ostergard, M. J. L., C Clausen, C Bagger, M Mogensen. Manganite-zirconia composite cathodes for SOFC: influence of structure and composition, Electrochim. Acta. 40 (1995) 1971-1981.

[2] BA Boukamp. Interpretation of an 'inductive loop' in the impedance of an oxygen ion conducting electrolyte/metal electrode system, Solid State Ionics. 143 (2001) 47-55.

[3] GO Lauvstad, R Tunold, S Sunde. Electrochemical oxidation of CO on Pt and Ni point electrodes in contact with an yttria-stabilized zirconia electrolyte - II. Steady-state and impedance measurements, J.Electrochem.Soc. 149 (2002) E506-E514.

[4] J Newman. Resistance of flow of current to a disk, J. Electrochem. Soc. 113 (1966) 501-502.

[5] M Nagata, Y Itoh, H Iwahara. Dependence of observed voltages on the positioning of the reference electrode on the solid electrolyte, Solid State Ionics. 67 (1994) 215-224.

[6] T Kato, A Momma, Y Kaga, S Nagata, Y Kasuga, M Kitase. Influence of cell configuration on measuring interfacial impedances between a solid electrolyte and an electrode, Solid State Ionics. 132 (2000) 287-295.

[7] SB Adler, BT Henderson, MA Wilson, DM Taylor, RE Richards. Reference electrode placement and seals in electrochemical oxygen generators, Solid State Ionics. 134 (2000) $35-42$.

[8] G Prentice, Electrochemical Engineering Principles, first ed., Prentice-Hall, Englewood Cliffs, NJ, 1991. See p. 126-127.

[9] GW Coffey, LR Pederson, DM Ponds, PC Rieke, EC Thomsen. Fabrication and characterization of a novel Luggin capillary reference electrode for use in solid state electrolytes, Solid State Ionics. 178 (2007) 827-832.

[10] JO Bockris, Reddy, A. K. N., M Gamboa-Aldeco, Modern Electrochemistry 2A: Fundamentals of Electrodics, Second ed., Kluwer Academic/Plenum Publishers, New York, 2000. See p. 1108.

[11] G Hsieh, SJ Ford, TO Mason, LR Pederson. Experimental limitations in impedance spectroscopy: Part I - simulation of reference electrode artifacts in three-point measurements, Solid State Ionics. 91 (1996) 191-201.

[12] G Hsieh, TO Mason, LR Pederson. Experimental limitations in impedance spectroscopy: Part II - electrode artifacts in three-point measurements on Pt/YSZ, Solid State Ionics. 91 (1996) 203-212. 
[13] G Hsieh, TO Mason, EJ Garboczi, LR Pederson. Experimental limitations in impedance spectroscopy: Part III - effect of reference electrode geometry/position, Solid State Ionics. 96 (1997) 153-172.

[14] G Hsieh, SJ Ford, TO Mason, LR Pederson. Experimental limitations in impedance spectroscopy: Part VI - four point measurements of solid state materials systems, Solid State Ionics. 100 (1997) 297-311.

[15] J Winkler, PV Hendriksen, N Bonanos, M Mogensen. Geometric requirements of solid electrolyte cells with a reference electrode, J.Electrochem.Soc. 145 (1998) 11841192.

[16] SB Adler. Reference electrode placement in thin solid electrolytes, J. Electrochem. Soc. 149 (2002) E166-E172.

[17] J Rutman, I Riess. Placement of reference electrode in solid electrolyte cells, Electrochim. Acta. 52 (2007) 6073-6083.

[18] J Rutman, I Riess. Placement of reference electrode in solid electrolyte cells, Solid State Ionics. 179 (2008) 913-918.

[19] J Rutman, I Riess. Reference electrodes for thin-film solid-state ionic devices, Solid State Ionics. 179 (2008) 108-112.

[20] MJ Jorgensen, S Primdahl, M Mogensen. Characterization of composite SOFC cathodes using electrochemical impedance spectroscopy, Electrochim. Acta. 44 (1999) 4195-4201.

[21] Z Liu, JS Wainright, W Huang, RF Savinell. Positioning the reference electrode in proton exchange membrane fuel cells: calculations of primary and secondary current distribution, Electrochim. Acta. 49 (2004) 923-935.

[22] AJ Bard, LR Faulkner, Electrochemical Methods: Fundamentals and Applications, second ed., Wiley, New York, NY, 2001.

[23] A Hashibon, S Raz, I Riess. Preferred position for the reference electrode in solid state electrochemistry, Solid State Ionics. 149 (2002) 167-176.

[24] LA Dunyushkina, YX Lu, SB Adler. Microelectrode array for isolation of electrode polarization on planar solid electrolytes, J. Electrochem. Soc. 152 (2005) A1668-A1676.

[25] DY Wang, AS Nowick. Cathodic and Anodic Polarization Phenomena at PlatinumElectrodes with Doped Ceo2 as Electrolyte .2. Transient Overpotential and Ac Impedance, J.Electrochem.Soc. 126 (1979) 1166-1172.

[26] H Gerischer. Z. Phys. Chem. 198 (1951) 216. 
[27] BA Boukamp, Bouwmeester, H. J. M. Interpretation of the Gerischer impedance in solid state ionics, Solid State Ionics. 157 (2003) 29-33.

[28] J Park, RN Blumenthal. Electronic Transport in 8 mole percent $\mathrm{Y}_{2} \mathrm{O}_{3}-\mathrm{ZrO}_{2}$, J. Electrochem. Soc. 136 (1989) 2867-2876.

[29] SB Adler. Mechanism and kinetics of oxygen reduction on porous $\mathrm{La}_{1-\mathrm{x}} \mathrm{Sr}_{\mathrm{x}} \mathrm{CoO}_{3 \text {-delta }}$ electrodes, Solid State Ionics. 111 (1998) 125-134.

[30] COMSOL AB, COMSOL Multiphysics Users Guide, version 3.2 (2005) 622.

[31] AC West, J Newman. Interpretation of kinetic rate data taken in a channel flow cell, J. Electrochem. Soc. 136 (1989) 3755-3759.

[32] G Fafilek. The use of voltage probes in impedance spectroscopy, Solid State Ionics. 176 (2005) 2023-2029.

[33] DM Reed, HU Anderson, W Huebner. Characterization of solid oxide fuel cells by use of an internal Pt voltage probe, J. Electrochem. Soc. 143 (1996) 1558-3759.

[34] S McIntosh, SB Adler, JM Vohs, RJ Gorte. Effect of polarization on and implications for characterization of LSM-YSZ composite cathodes, Electrochemical and Solid State Letters. 7 (2004) A111-A114.

[35] C Gabrielli, Identification of electrochemical processes by frequency response analysis, Technical Report \#004/83, Solartron Analytical, Farnborough, UK, 1984, pp. 53-78.

[36] D Applebaum. Lévy processes - from probability to finance and quantum groups, Notices of the AMS. 51 (2004) 1336-1347. 


\section{CHAPTER 3}

\section{DEVELOPMENT AND FABRICATION OF GADOLINIA-DOPED CERIA ELECTRODE SOLID OXIDE ELECTROLYSIS CELLS}

\subsection{Introduction}

In Chapter 1, the choice of gadolinium-doped ceria (GDC) as the MEIC electrode material for this study was briefly discussed. In this chapter, the choice of gadoliniumdoped ceria (GDC) and dopant type and level is described in more depth along with a review of the available material properties influencing this choice. The remaining part of this chapter discusses the fabrication procedures for the GDC electrode solid oxide cells, and the experimental setup for making the Electrochemical Impedance Spectroscopy (EIS) measurements on the cells.

\subsection{Choice of $\mathbf{4 0} \mathbf{m o l} \%$ gadolinium-doped ceria as cathode for carbon dioxide reduction \\ 3.2.1 Background}

The choice of an electrode material for a solid oxide electrochemical device encompasses a number of physical and chemical factors and characteristics. Besides the mixed electronic-ionic conduction requirement discussed in Chapter 1, the electrode material must be thermodynamically stable over wide ranges of temperature and oxygen partial pressure, chemically compatible with the electrolyte, both during sintering or other 
fabrication processes and during temperature operation, a close thermal expansion coefficient match with the electrolyte material, and finally, high electrocatalytic activity for the reaction of interest [1]. In this section, the rationale of why GDC was chosen as a potential carbon dioxide reduction cathode will be provided along with a review of the relevant physical properties of this electrode material.

Ceria has been extensively looked at over the past 15 years as a solid oxide electrolyte [2] mainly due to its higher ionic conductivity at lower temperatures than YSZ, the more widely used solid oxide electrolyte. In particular, rare earth-doped ceria (i.e. $\mathrm{M}_{x} \mathrm{Ce}_{1-x} \mathrm{O}_{1.95-\delta}$, where $\mathrm{M}=\mathrm{Sm}, \mathrm{Gd}, \mathrm{Sc}, \mathrm{La}, \mathrm{Mg}, \mathrm{Ca}$, or $\mathrm{Y}$ ) has been studied extensively, since the ionic conductivity is improved over pure ceria by the introduction of trivalent or divalent cations in place of the $\mathrm{Ce}^{4+}$ cation, while the electronic conductivity is reduced by varying degrees, depending on the dopant [3].

Cerium has a $4 \mathrm{f}^{2} 5 \mathrm{~d}^{0} 6 \mathrm{~s}^{2}$ electron configuration in its outer shells and exhibits both the +3 and +4 oxidation states; it begins to oxidize to cerium dioxide, $\mathrm{CeO}_{2}$, at oxygen partial pressures above $10^{-40}$ atm [4]. $\mathrm{CeO}_{2}$ crystallizes in the fluorite structure forming a face-centered-cubic (FCC) unit cell with each cerium atom having a coordination number of 8. This fluorite structure tends to allow a large tolerance for atomic disorder, introduced by either reduction or doping with an aliovalent cation (i.e. a cation with a different oxidation state than the host cation). 


\subsubsection{Oxygen non-stoichiometry of ceria and doped ceria}

$\mathrm{CeO}_{2}$ undergoes partial reduction under a reducing atmosphere. For example in a $\mathrm{CO}-\mathrm{CO}_{2}$ gas mixture the reduction of $\mathrm{CeO}_{2}$ can be described, using Kröger-Vink [5] notation $^{*}$ :

$2 C e_{C e}^{x}+O_{O}^{x}+C O \rightleftharpoons 2 C e_{C e}^{\prime}+V_{O}^{\bullet \bullet}+C O_{2}$

This reduction process causes the material to lose oxygen to the ambient reducing atmosphere, creating vacancies at oxygen sites in the ceria lattice. The oxygen nonstoichiometry of ceria has been measured by a number of workers for both pure $\mathrm{CeO}_{2}[6]$ and doped $\mathrm{CeO}_{2}$ [7-9]; a review of the data is provided by Mogensen et al. [3]. Unfortunately, there are few data available in the literature for doping levels greater than $20 \mathrm{~mol} \%$ GDC [10].

Oxygen non-stoichiometry can also be increased by doping ceria. The introduction of an aliovalent dopant such as $\mathrm{Gd}^{+3}$ or $\mathrm{Sm}^{+3}$ in $\mathrm{CeO}_{2}$ produces negatively charged defects, increasing the vacancy concentration in the oxide:

$\mathrm{Gd}_{2} \mathrm{O}_{3} \stackrel{\mathrm{CeO}_{2}}{\longrightarrow} 2 \mathrm{Gd}_{\mathrm{Ce}}^{\prime}+\mathrm{V}_{\mathrm{O}}^{\bullet \bullet}+3 \mathrm{O}_{\mathrm{O}}^{x}$

\footnotetext{
${ }^{*}$ See Appendix G for an explanation of Kröger-Vink notation.
} 
Doped ceria, similar to pure $\mathrm{CeO}_{2}$, also loses oxygen under reducing conditions, via the oxygen non-stoichiometry shown in Equation (3-1). In this work, these two contributions to oxygen non-stoichiometry will be denoted as follows:

For $G d_{x} C e_{1-x} O_{2-\delta}: \quad \delta=\delta_{0}+\delta^{\prime}=\frac{x}{2}+\delta^{\prime}$

where $\delta_{0}=$ deviation from stoichiometry due to Gd doping mole fraction

$\delta^{\prime}=$ deviation from stoichiometry due to $P_{O_{2}}$

$\delta=$ total deviation in oxygen non-stoichiometry

In general, the change in oxygen non-stoichiometry due to the reducing atmosphere, $\delta^{\prime}$, is smaller for doped ceria than for pure $\mathrm{CeO}_{2}$. Figure 3-1 shows the oxygen non-stoichiometry for several Gd doping levels, and indicates a decrease in $\delta^{\prime}$ with increasing doping level for a given temperature and $P_{\mathrm{O}_{2}}$ level.

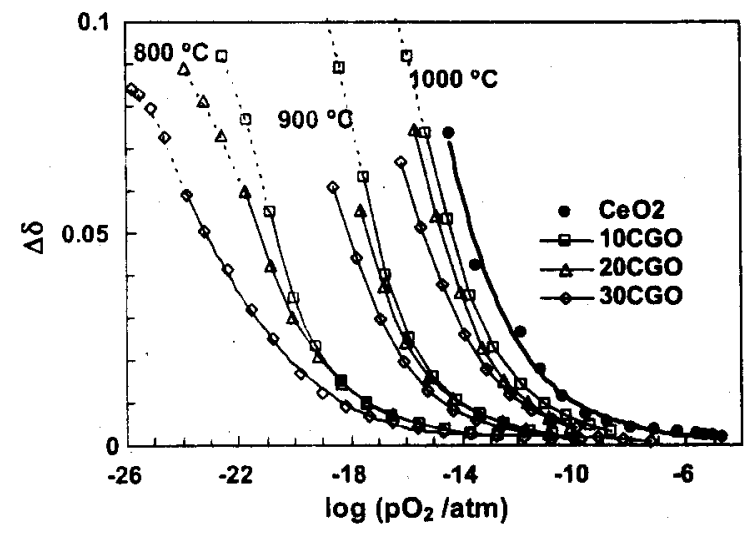

Figure 3-1. Oxygen non-stoichiometry data for pure ceria and gadolinia-doped ceria, $x=$ $0.10,0.20$, and 0.30 . From reference [11]. $\Delta \delta$ ordinate is denoted as $\delta^{\prime}$ in this work. 


\subsubsection{Ionic conductivity properties of ceria and doped ceria}

Ionic conductivity in a number of solid oxide type materials like ceria has been known for over 100 years [12]. Pure cerium oxide is actually a poor ionic conductor $\left(\sigma_{\mathrm{i}}\right.$ $=1.1 \times 10^{-5} \mathrm{~S} / \mathrm{cm} @ 600^{\circ} \mathrm{C}$ in air) [13], compared to the commonly used electrolyte, YSZ $\left(\sigma_{\mathrm{i}} \sim 10^{-4} \mathrm{~S} / \mathrm{cm} @ 600^{\circ} \mathrm{C}\right)$, but as discussed in the previous section, the vacancy concentration can be increased by doping ceria with a lower valence cation, enhancing the ionic conductivity.

In early studies of the conductivity of doped oxides, researchers $[14,15]$ observed an increase in ionic conductivity for Gd levels up to $x=0.10$. For $x>0.10$, they reported: 1) the ionic conductivity for doped GDC decreases with increasing dopant concentration, and 2) the activation energy for GDC ionic conductivity increases with dopant concentration. But in more recent work, Dikman et al. [13] reported the ionic conductivity of GDC increases with doping level and reaches a maximum at $x=0.25$, with a corresponding minimum for the activation energy. The decrease in ionic conductivity and increase in activation energy for $x>0.25$ is thought to be caused by the formation of defect association pairs [16]:

$$
G d_{C e}^{\prime}+V_{o}^{\bullet \bullet} \rightarrow\left\{G d_{C e}^{\prime} V_{o}^{\bullet \bullet}\right\}^{\bullet}
$$

Table 3-1 gives a sample set of ionic conductivities of pure and Gd-doped ceria at several doping levels for a temperature and $P_{\mathrm{O}_{2}}$ level applicable to the experimental conditions for the impedance measurements in this work (see section 3.4). 


$\begin{array}{lll}\text { Composition } & \begin{array}{l}\text { Electronic conductivity } \\ \sigma_{\mathrm{e}}\left(\mathrm{S} \mathrm{cm}^{-1}\right)\end{array} & \text { Reference } \\ \mathrm{CeO}_{2} & 2.72 & {[1]} \\ \mathrm{Gd}_{0.2} \mathrm{Ce}_{0.8} \mathrm{O}_{1.9} & 0.53 & {[11]} \\ \mathrm{Gd}_{0.3} \mathrm{Ce}_{0.7} \mathrm{O}_{1.9} & 0.35 & {[11]} \\ \mathrm{Gd}_{0.4} \mathrm{Ce}_{0.6} \mathrm{O}_{1.8} & 0.33 & {[1]} \\ & & \\ & \text { Ionic conductivity } & \\ \mathrm{CeO}_{2} & \sigma_{\mathrm{i}}\left(\mathrm{S} \mathrm{cm}^{-1}\right) & \\ \mathrm{Gd}_{0.2} \mathrm{Ce}_{0.8} \mathrm{O}_{1.9} & 0.07 & {[3]} \\ \mathrm{Gd}_{0.4} \mathrm{Ce}_{0.6} \mathrm{O}_{1.8} & 0.36 & {[1]} \\ & 0.08 & {[1]}\end{array}$

Table 3-1. Some representative electronic and ionic conductivities of ceria and Gd-doped ceria at $1000^{\circ} \mathrm{C}$ and $P_{O_{2}}=10^{-15} \mathrm{~atm}$.

$\begin{array}{llc}\text { Composition } & \begin{array}{l}\text { Electron mobility } \\ \left(\mathrm{cm}^{2} \mathrm{~V}^{-1} \mathrm{~s}^{-1}\right)\end{array} & \begin{array}{l}\text { Ionic mobility } \\ \left(\mathrm{cm}^{2} \mathrm{~V}^{-1} \mathrm{~s}^{-1}\right)\end{array} \\ \mathrm{CeO}_{2} & 8 \times 10^{-3} & - \\ \mathrm{Gd}_{0.1} \mathrm{Ce}_{0.9} \mathrm{O}_{1.95} & 5.14 \times 10^{-3} & 4.86 \times 10^{-4} \\ \mathrm{Gd}_{0.2} \mathrm{Ce}_{0.8} \mathrm{O}_{1.9} & 2.91 \times 10^{-3} & 2.86 \times 10^{-4}\end{array}$

Table 3-2. Example electronic and ionic mobilities for several compositions of ceria at $1000^{\circ} \mathrm{C}$ and $P_{O_{2}} \approx 10^{-12}$ atm. Data are from reference [17].

\subsubsection{Electronic properties of ceria and doped ceria}

Ceria and ceria-based oxides have significant n-type electronic conduction at high temperatures and low $P_{\mathrm{O}_{2}}$ [18]. In ceria, electronic conduction occurs due to the formation of small polarons, where the electron is self-trapped at the $\mathrm{Ce}^{+3}$ lattice site, and can move to an adjacent site by an activated "hopping" process similar to ionic diffusion [4]. Because of this, the electron mobility in ceria is significantly lower, i.e. on the order 
of $10^{-4}-10^{-2} \mathrm{~cm}^{2} / \mathrm{V}-\mathrm{sec}\left(\right.$ at $1000^{\circ} \mathrm{C}$ ), compared to $1350 \mathrm{~cm}^{2} / \mathrm{V}-\mathrm{s}$ [19] for a typical semiconductor material like $\mathrm{Si}\left(\right.$ at $\left.25^{\circ} \mathrm{C}\right)$.

Because of the lower valence of the doped cation, an increase in the doping level of GDC decreases the conduction electron concentration [20] and consequently the electronic conductivity. For example, the total concentration of electrons is $1.81 \times$ $10^{21} / \mathrm{cm}^{3}$ for $20 \mathrm{~mol} \% \mathrm{Gd}$-doped ceria and $1.48 \times 10^{21} / \mathrm{cm}^{3}$ for $30 \mathrm{~mol} \% \mathrm{Gd}$-doped ceria [20] based on calculations from thermogravimetry measurements. The associated reduction in electronic conductivity and electron mobility can be seen in the example values in Tables 3-1 and 3-2. The decrease in electronic conductivity is more ideal for the electrolyte applications for doped ceria, but not necessarily for an electrode material application.

$\begin{array}{ll}\text { Composition } & \begin{array}{l}\text { Thermal expansion } \\ \text { coefficient (TEC) } \\ 10^{-6} \mathrm{~K}^{-1}\end{array} \\ \mathrm{CeO}_{2} & 12.3 \\ \mathrm{Gd}_{0.2} \mathrm{Ce}_{0.8} \mathrm{O}_{1.9} & 12.5 \\ \mathrm{Gd}_{0.4} \mathrm{Ce}_{0.6} \mathrm{O}_{1.8} & 12.1 \\ \mathrm{Y}_{0.15} \mathrm{Zr}_{0.85} \mathrm{O}_{1.925} & 10.8\end{array}$

Table 3-3. Thermal expansion coefficient (TEC) data for pure ceria and selected Gd doping levels. TEC for YSZ included for comparison. Data are from reference [1]. 


\subsubsection{Thermal and oxygen nonstoichiometry expansion considerations}

The thermal expansion coefficient (TEC) of doped ceria is also dependent on the doping level; for an electrode material, it should have a fairly close match with the electrolyte material, which is YSZ for our application. Table 3-3 contains a brief list of TEC data for GDC and indicates the TEC tends to increase with doping level. The 40 mol\% GDC at a TEC of $12.1 \times 10^{-6} \mathrm{~K}^{-1}$, is the closest match to the $10.8 \times 10^{-6} \mathrm{~K}^{-1}$ value for YSZ.

In addition, ceria and doped ceria expand under low $P_{\mathrm{O}_{2}}$ conditions due to expansion of the fluorite lattice as $\mathrm{Ce}^{4+}$ is reduced to $\mathrm{Ce}^{3+}$. This "chemical expansivity" [21] of ceria under reducing conditions can be lowered with higher doping concentrations of the aliovalent cation. Chemical expansivity data for GDC, like TEC data, are sparse, but Mogensen and Mogensen [22] did make dilatometry measurements on $40 \mathrm{~mol} \% \mathrm{Gd}-$ doped ceria and reported less than $1 \%$ expansion at $1000^{\circ} \mathrm{C}$ and $P_{O_{2}}$ in the range of $10^{-17}$ $10^{-18} \mathrm{~atm}$, i.e. similar to our test conditions. As a comparison, they reported a value over $2 \%$ on pure $\mathrm{CeO}_{2}$ under the same test conditions.

Because of the very limited GDC TEC and chemical expansivity data, a simple qualitative test was performed by brush-painting GDC ink (formulation described in section 3.3) for 10, 20, and $40 \mathrm{~mol} \%$ GDC onto an $8 \mathrm{~mol} \% \mathrm{YSZ}$ test coupon, and sintered at $1250^{\circ} \mathrm{C}$. This test coupon was then heated to $800^{\circ} \mathrm{C}$ and cooled under a reducing atmosphere $\left(\mathrm{CO} / \mathrm{CO} 2\right.$ ratio $=0.05, P_{O_{2}}=10^{-16}$ atm $)$ and a "scratch" test performed, i.e. 
each of the GDC sintered layers were scraped with a spatula. The $40 \mathrm{~mol} \%$ GDC layer had the best adherence, and exhibited very minor cracking.

\subsubsection{Electrocatalytic activity for oxidation of hydrocarbons}

Ceria is widely used as a catalyst support for oxidation of carbon monoxide and other hydrocarbons in automotive three-way catalytic converters due to its oxygen storage capacity [23]. In recent years, a number of investigations have examined rareearth doped ceria or ceria-containing composites as an anode for direct hydrocarbon oxidation fuel cells [24-27] because of its mixed-conducting properties under reducing anode conditions, and its proven catalytic activity for oxidation of hydrogen, carbon monoxide, methane, and other hydrocarbons. This latter property suggests that doped ceria may also be electrocatalytically active for carbon dioxide reduction. Further support of this hypothesis is a thermogravimetry study of gadolinia-doped ceria in CO$\mathrm{CO}_{2}$ (and $\mathrm{H}_{2}-\mathrm{H}_{2} \mathrm{O}$ ) reducing atmospheres, which suggests its $\mathrm{CO}-\mathrm{CO}_{2}$ exchange kinetics are comparable in magnitude to $\mathrm{H}_{2}-\mathrm{H}_{2} \mathrm{O}$ exchange kinetics [28].

There has been some debate as to whether doped ceria improves the electrocatalytic activity of hydrocarbon oxidation compared to pure ceria. McIntosh et. al. [29] reported that pure ceria showed a higher catalytic activity than Sm-doped ceria for butane oxidation, attributing it to the greater oxygen capacity of pure ceria, but Mogensen et. al. [30] noted that pure ceria is in a reduced state in a typical SOFC anode application, which would diminish this oxygen capacity effect. They [31] separately made impedance measurements on cone electrodes in $\mathrm{CH}_{4}$ and $3 \% \mathrm{H}_{2} \mathrm{O}$, and reported lower area-specific- 
resistance (ASR) values for GDC ( $x=0.1,0.2$ and 0.4$)$ compared to pure ceria, indicating GDC has a higher electro-catalytic activity for $\mathrm{CH}_{4}$ oxidation.

Although none of these references suggests an optimum doping level, the more extensive work on oxygen reduction on the perovskite class of oxides indicates that higher vacancy concentrations [32] seem to correlate well with faster oxygen ion transport and oxygen exchange on these mixed conductors. This suggests that higher doped GDC may potentially have higher catalytic activity for the reduction of $\mathrm{CO}_{2}$.

\subsubsection{Discussion of multiple phases in GDC at the 40 mol\% doping level}

There is some uncertainty as to the complete solubility of gadolinia in ceria at the higher doping levels, and some of the early work with doped ceria is conflicting. Etsell and Fenglas [33] reported that $\mathrm{Gd}_{2} \mathrm{O}_{3}$ is completely soluble in $\mathrm{CeO}_{2}$, whereas Kudo and Obayashi [34] and Tianshu et al. [35] concluded that it forms a monophasic solid solution having the fluorite phase at least up to a mole fraction of 0.4 . Grover and Tyagi [36] studied this system over the range of $x=0.05-0.95$ and reported GDC takes on the fluorite structure up to $x=0.4$, gradually changing to a C-type (bcc) solid solution with no miscibility gap or biphasic regions. And, as noted in previous sections of this chapter, a number of workers $[10,22,37]$ have reported oxygen non-stoichiometry and thermal expansion properties for $40 \mathrm{~mol} \% \mathrm{GDC}$, without indicating if this formulation is of a single phase at this doping level. But, two very recent works [38,39] have reported the detection of minor secondary phases in Gd-doped ceria for $x_{G d}>0.3$. 
For the experimental portion of this work, $\mathrm{Gd}_{0.4} \mathrm{Ce}_{0.6} \mathrm{O}_{1.8}$ powder (NextTech Materials, surface area $=17.549 \mathrm{~m}^{2} / \mathrm{gm}$ ) was purchased, synthesized using the solid-state reaction method. An initial routine x-ray powder diffraction (XRD) analysis of this powder, using a conventional diffractometer with focusing geometry and a proportional point detector, indicated it was a single phase material. But, because of the findings mentioned above, a more detailed XRD analysis was performed on the powder. This second set of XRD data was gathered on a Bruker D8 Discover diffractometer with a proportional multiwire area detector using $\mathrm{Cr} \mathrm{K}_{\alpha}$ radiation $\left(\lambda_{\mathrm{K} \alpha 1}=2.289760 \AA, \lambda_{\mathrm{K} \alpha 2}=\right.$ 2.293663). The data for each sample consisted of five overlapping frames of $2 \mathrm{D}$ data. The exposure time for each frame was 3 hours. Each 2D frame was integrated about the axis of the direct beam in $0.025^{\circ} 2 \theta$ steps to produce a conventional $2 \theta$-intensity dataset. The $2 \theta$-intensity datasets for each frame were then combined to produce a final $2 \theta$ intensity plot that was used for analysis.

Figure 3-2 contains the XRD scan for this powder sample with the intensity peaks overlaid for the two PDF (Powder Diffraction File) cards that are the closest match for this material; a PDF card was not available for $\mathrm{Gd}_{0.4} \mathrm{Ce}_{0.6} \mathrm{O}_{1.80}$. The major phase closely matches the phase ID for $\mathrm{Gd}_{0.35} \mathrm{Ce}_{0.65} \mathrm{O}_{2}$ (PDF card \# 04-006-3415), with a slight negative offset in the peaks, especially the secondary peaks. This shift, due to a slightly lower Bragg angle [40], shows the Gd content is greater than $0.35\left(x_{\mathrm{Gd}}>0.35\right)$, and is consistent with the slightly larger lattice parameter expected for $\mathrm{Gd}_{0.4} \mathrm{Ce}_{0.6} \mathrm{O}_{1.80}$. A series of minor peaks confirm the existence of a second minor phase and closely match the peaks for $\left(\mathrm{Gd}_{0.6} \mathrm{Ce}_{0.4}\right)_{2} \mathrm{O}_{3.2}$ (PDF card \#00-046-0508). Given this minor phase is Gd-rich, the major fluorite phase must be slightly Gd-poor $\left(x_{\mathrm{Gd}}<0.40\right)$. 


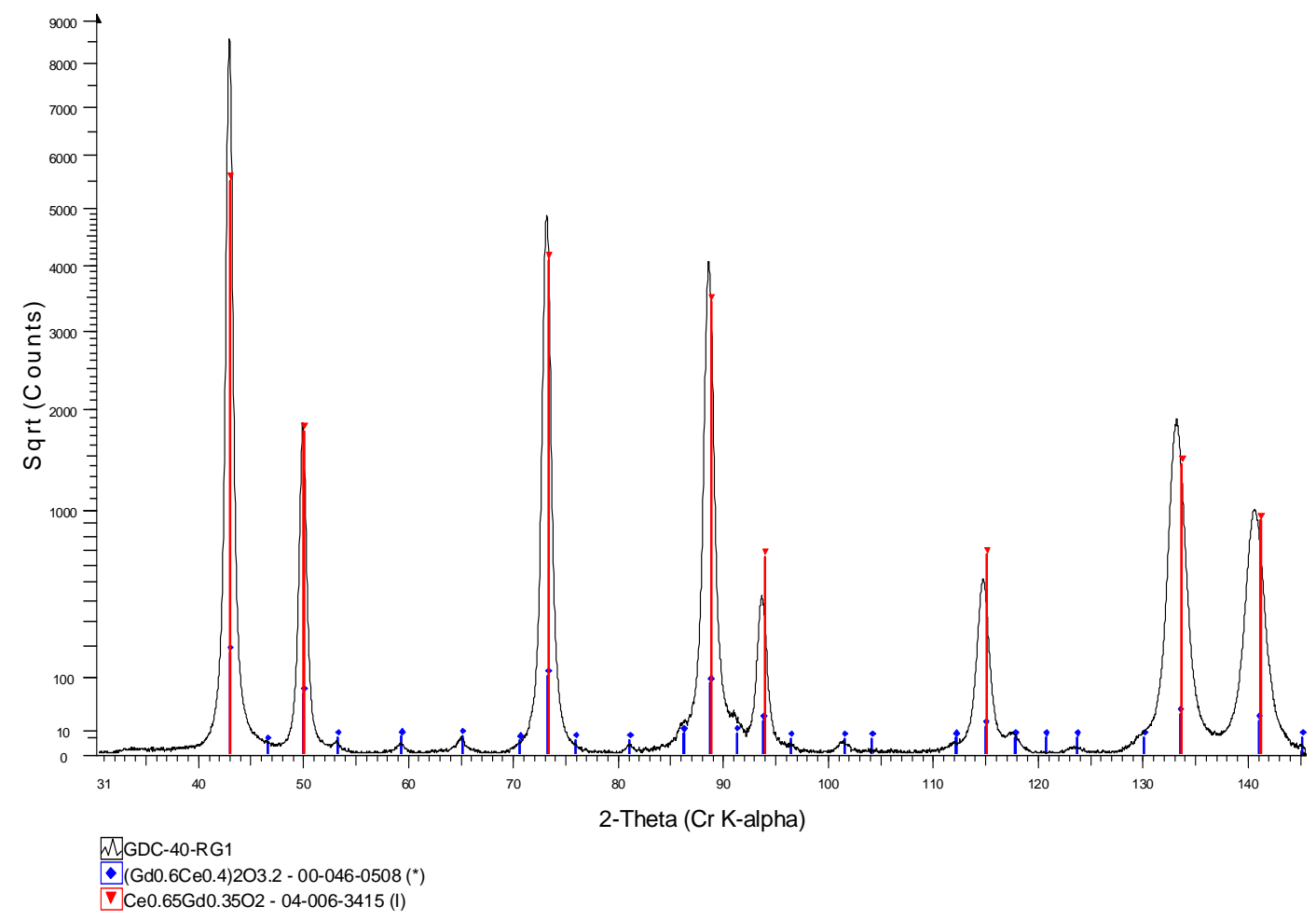

Figure 3-2. An X-ray diffraction (XRD) scan of the $40 \mathrm{~mol} \% \mathrm{Gd}$-doped $\mathrm{CeO}_{2}$ powder used to fabricate the GDC cells in this study. Note the ordinate scale is the square root of the intensity to emphasize the secondary peaks of the minor phase.

A lattice parameter of $5.4378 \pm 0.0001 \AA$ was determined from the data using a least-squares analysis, utilizing only the experimental data for the major phase. In order to compare the trend in lattice parameter with Gd doping level in ceria, lattice parameter data at lower doping levels from the PDF database were plotted with the measured value for our formulation, along with the following model fit for lattice parameters in rare-earth doped ceria developed by Hong and Virkar [41]: 
$a\left(x, r_{m}\right)=\frac{4}{\sqrt{3}}\left[x r_{G d}+(1-x) r_{C e}+(1-0.25 x) r_{O}+0.25 x r_{V_{o}^{*}}\right] \times 0.9971$

where $r_{C e}=0.97 \AA, r_{G d}=1.053 \AA, r_{O}^{2-}=1.38 \AA$, and $r_{V_{o}^{*}}=1.164 \AA$, are the effective radii for $\mathrm{Ce}, \mathrm{Gd}, \mathrm{O}$, and oxygen vacancy, respectively. This model fit assumes the doped ceria remains in the fluorite phase as the doping level is increased. More specifically, the fit assumes the oxygen vacancy radius remains constant with respect to dopant concentration and cation radius, the linear combination of host and dopant cations determine the effective radius of cation, and the linear combination of oxygen ion and oxygen vacancy determines the effective radius of the anion. The ionic radii values were compiled by Shannon [42] and the oxygen vacancy radius was calculated by Hong and Virkar [41] using experimentally determined lattice parameters for doped ceria compositions of $\mathrm{Gd}$ and various other rare-earth dopants. The 0.9971 value is an empirical factor added to correct Equation (3-5) for the experimentally measured lattice parameter of pure $\mathrm{CeO}_{2}$.

Figure 3-3 indicates our measured lattice parameter value is consistent with PDF card data measured at lower doping levels $(x=0.0-0.2)$ and the Hong and Virkar correlation, further indicating the major phase of our sample is $\mathrm{Gd}_{0.4} \mathrm{Ce}_{0.6} \mathrm{O}_{1.80}$. But the PDF card data and Hennings and Reimart data [39] for $\mathrm{x} \geq 0.3$ show a deviation from the Hong and Virkar correlation, emphasized in Figure 3-3 with a separate linear regression fit of these data. The Grover and Tyagi data deviate substantially from either correlation, but their $x=0.4$ value closely matches our measured value for the lattice parameter. 


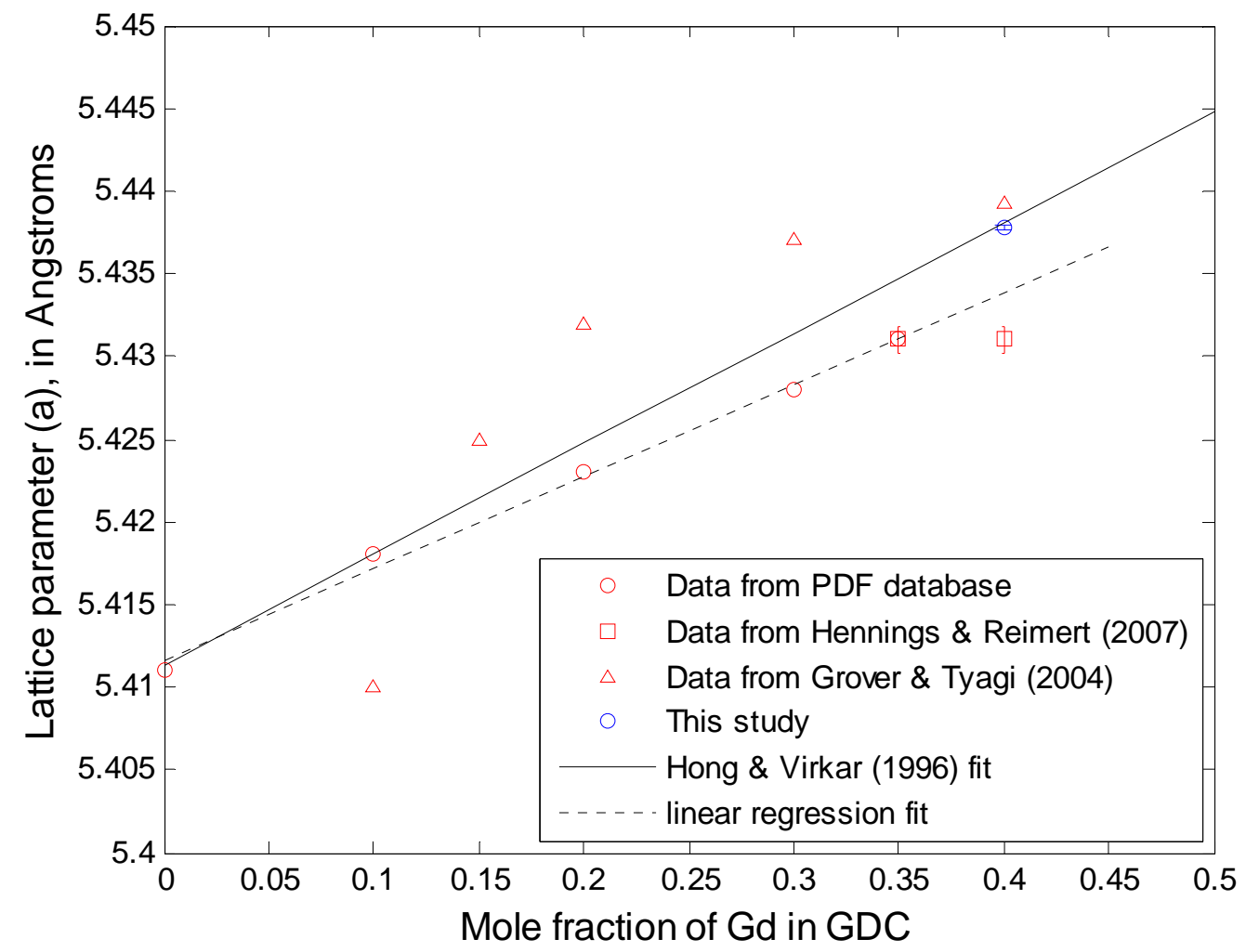

Figure 3-3. Measured lattice parameter for $40 \mathrm{~mol} \%$ Gd-doped ceria (in blue) along with lattice parameter data for pure ceria and at Gd-doping levels of 10-40 mol\% (in red). 40 mole $\% \mathrm{Gd}$-doped ceria powder for this study measured with $\mathrm{CuK} \alpha$ radiation in a Bruker D8 Discover diffractometer. Other data are from references [36,39] and the Powder Diffraction File (PDF) database (International Centre for Diffraction Data ${ }^{\circledR}$ ).

The deviation in Hennings and Reimart data may possibly be due to the calculation of the lattice parameter using the whole set of experimental XRD data, as opposed to segregating the data for the major phase. 


\subsubsection{Other considerations}

Finally, there are a few additional considerations in the choice of GDC as a mixedconducting cathode for $\mathrm{CO}_{2}$ reduction, when compared to Ni-YSZ cermet, a mixed conducting composite electrode with more extensive work as an anode for $\mathrm{CO}$ and hydrocarbon oxidation (reviewed in Chapter 1). These Ni-based SOFC electrodes have a few limitations that a ceria or doped ceria electrode may not exhibit. In particular, $\mathrm{Ni}$ composite electrode performance can be reduced due to exposure to sulfur [43], formation of carbon deposits under thermodynamically favorable conditions [44], and exposure to oxidation/reduction cycles. Although preliminary, there are some reported results that indicate ceria is tolerant to sulfur [45], and resistant to carbon deposit formation under hydrocarbon oxidation conditions [46]. And ceria, as a catalyst support in automobile catalytic converters, has demonstrated a tolerance to rapid oxidation/reduction cycles, but it remains to be seen if its chemical expansivity under highly reducing conditions can be appropriately matched to other SOFC components, namely electrolyte and current collector materials.

\subsubsection{Summary}

With these material properties in mind, Gd-doped ceria at a doping level of 40 mol\% was chosen for the electrode material. This is consistent with the recommendation of Mogensen et al. [1] that the best composition for ceria-based anodes is a $40 \%-50 \%$

substitution of the $\mathrm{Ce}^{+4}$ with a +3 valent ion such as $\mathrm{Y}^{+3}, \mathrm{Sm}^{+3}$ or $\mathrm{Gd}^{+3}$. The dimensional stability of ceria (i.e. the thermal and chemical expansion mismatch with YSZ) was the primary factor for the choice of the $40 \mathrm{~mol} \%$ doping level. Mogensen et al. [25,47] 
reported problems with adherence of the electrode material to the YSZ electrolyte and recommended the $40 \mathrm{~mol} \%$ GDC despite the lower ionic and electronic conductivities at this higher doping level. Attempts at fabrication of cells at the 10 and 20 mol\% doping levels as part of this work resulted in similar YSZ electrolyte adherence problems, along with adherence issues with the Pt current collector.

\subsection{Fabrication of the solid oxide symmetric cells}

\subsubsection{Fabrication of solid oxide electrolyte pellets}

Symmetric SOE cells were fabricated using the pellet cell geometry in Figure 2-3. For the electrolyte, 8 mole \% yttria stabilized zirconia (YSZ) powder (Tosoh TZ8Y) was uniaxially cold-pressed at $29 \mathrm{MPa}$ into pellets using a 31-mm cylindrical die. The pellet die surfaces were swabbed with a saturated solution of stearic acid in acetone and allowed to air dry prior to each press; the resulting thin coat of dry stearic acid served as a lubricant [48], aiding in removal of the pressed pellet. The utmost care -- cleaning die with dionized water after each press, handling pellet with latex gloves, etc -- was used in maintaining cleanliness of the electrode/electrolyte surface to avoid contamination via silica or salts. Some workers [49-51] have detected migration of $\mathrm{Si}^{4+}, \mathrm{Na}^{+}, \mathrm{Al}^{3+}$, and other ions to the electrode/electrolyte interface and have measured increased interface resistances for samples with these contaminants purposely added, indicating these contaminants can have a detrimental effect on cell performance.

The pellets were sintered in two stages. First, they were bisqued, or partially sintered, at $1000^{\circ} \mathrm{C}$ for 1 hour using a heating and cooling ramp rate of $1{ }^{\circ} \mathrm{C} / \mathrm{min}$. A hole 
$2.5 \mathrm{~mm}$ deep was then drilled into the center of one pellet face, by hand-turning a standard $1 / 8$ in. $(3 \mathrm{~mm})$ diameter drill bit.

In the second step, the pellets were sintered at $1500^{\circ} \mathrm{C}$ for 4 hours using a heating ramp rate of $1^{\circ} \mathrm{C} / \mathrm{min}$ and cooling rate of $3^{\circ} \mathrm{C} / \mathrm{min}$. The final pellet diameter was typically $22.8 \mathrm{~mm}$ with a thickness of $4.6 \mathrm{~mm}$. The reference electrode hole was typically $2.3 \mathrm{~mm}$ deep with an inside diameter allowing the snug fit of a $2.4 \mathrm{~mm}$ diameter alumina tube containing the reference electrode Pt wire. The density was measured using the Archimedes method and the pellet densities achieved were greater than $97 \%$ of the $6.01 \mathrm{gm} / \mathrm{cm}^{3}$ theoretical value for $8 \mathrm{~mole} \%$ YSZ [52].

Before applying the electrode via screen-printing or spray-painting, the electrolyte pellet was rinsed with distilled water and allowed to air dry. No special processing (e.g. grinding or polishing) of the electrode/electrolyte surface was performed, even though electrolyte surface morphology can strongly effect electrode polarization and performance [53]. SEM was performed on several cells to qualitatively confirm the surface morphology was consistent between pellets; SEM images for a typical unpolished YSZ surface are shown in Figure 3-4. The surface roughness was on the order of $5 \mu \mathrm{m}$ and the grain size varies from $1-3 \mu \mathrm{m}$ in diameter with nearly complete sintering together of most particles. 


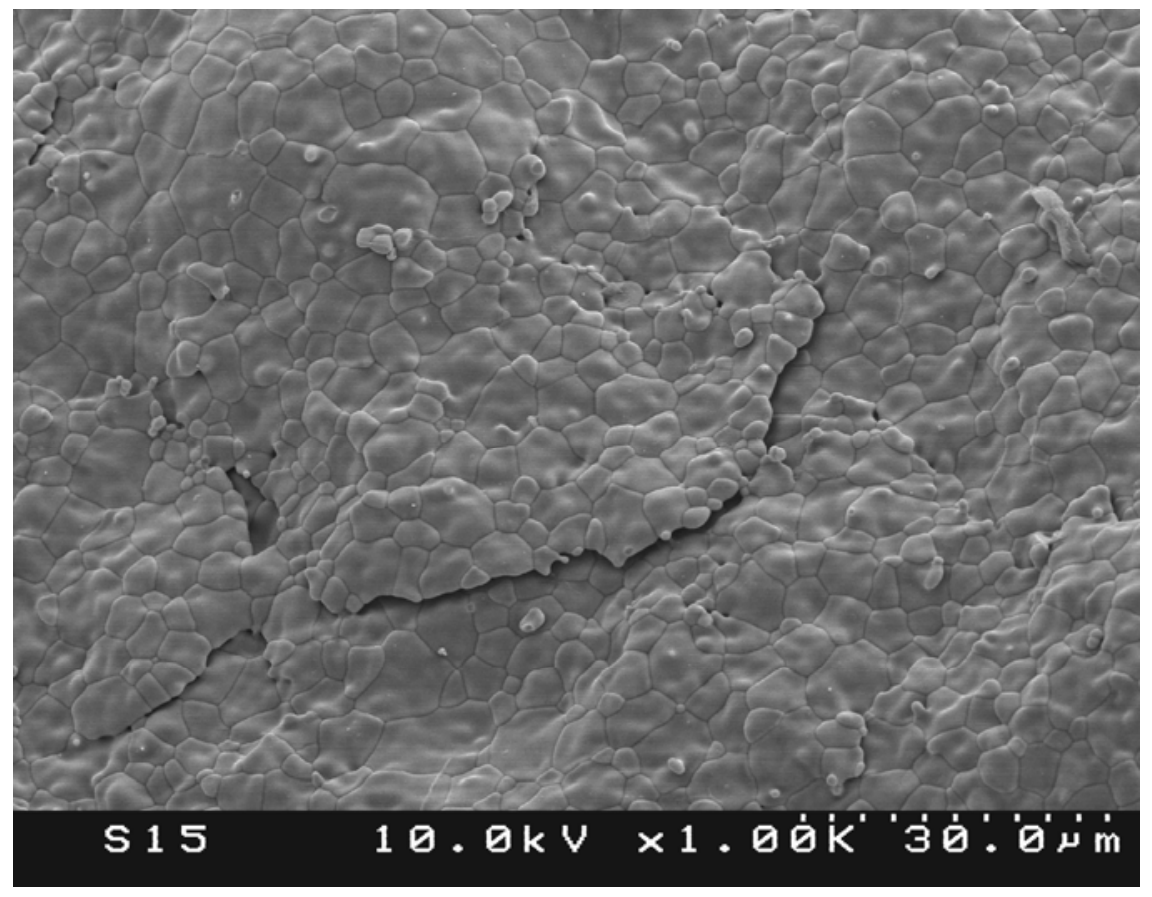

(a)

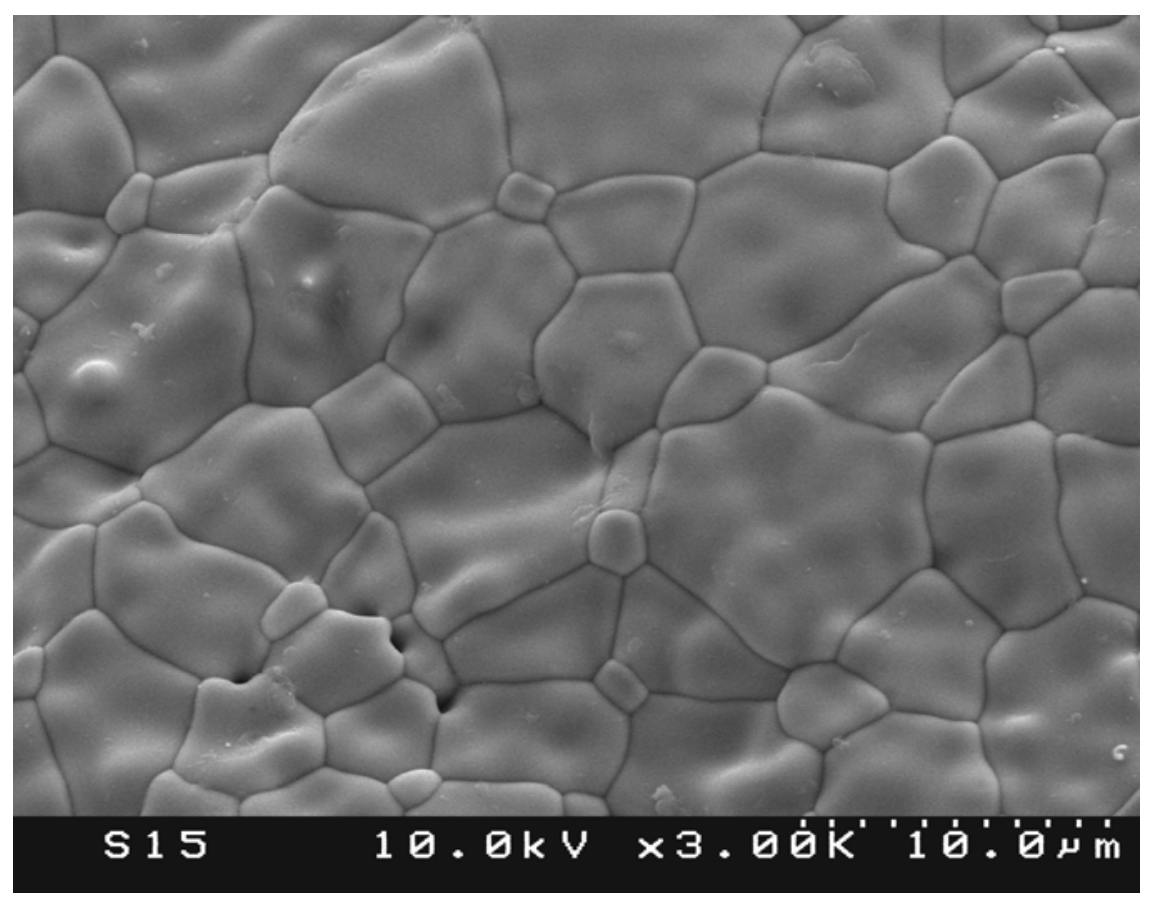

(b)

Figure 3-4. Scanning electron micrographs of the YSZ electrolyte surface of a diecast pellet. Sintered grain size is $\sim 2-6 \mu \mathrm{m}$ in diameter. As discussed in the text, the surface was minimally processed, i.e. no grinding or polishing of the electrode face surfaces. 


\subsubsection{Fabrication of 40 mol\% Gd-doped ceria $\left(\mathrm{Ce}_{0.6} \mathrm{Gd}_{0.4} \mathrm{O}_{1.95-\delta}\right)$ electrodes}

As mentioned in section 3.2, 40 mol\% Gd-doped ceria powder (NextTech

Materials, surface area $=17.5 \mathrm{~m}^{2} / \mathrm{gm}$ ) synthesized using the solid-state reaction method, was purchased for fabrication of the electrodes. The Gd-doped ceria powder was mixed with $\alpha$-terpineol and ethyl cellulose (ACROS Organics) on a 3-roll mill to make an ink; a small amount of oleic acid (Aldrich) was added as a dispersant [48]. The ink was diluted with xylene and ball-milled for 24 hours, using polytetrafluoroethylene (PTFE) balls to minimize grinding of the carbon or GDC powders. Glassy carbon powder $(0.4-12 \mu \mathrm{m}$ diameter, type 1, Alfa Aesar) was added as a pore former at $10 \mathrm{wt} \%$ of the GDC powder. This mixture was ultrasonically agitated for 2 minutes and ball-milled for 48 hours. The resulting GDC paint was air-brushed onto both faces of the electrolyte pellet, applying 35 coats using an $18 \mathrm{~mm}$ diameter cellophane tape mask. The area of each electrode was $2.5 \mathrm{~cm}^{2}$. The painted electrodes were dried in an oven for 8 hours at $70^{\circ} \mathrm{C}$. The "green" electrodes were heated in air with a ramp rate of $2^{\circ} \mathrm{C} / \mathrm{min}$ to $700^{\circ} \mathrm{C}$, held for 1 hour to burn out the pore former, then sintered for 2 hours at $1250^{\circ} \mathrm{C}$ with heating and cooling ramp rates of $2^{\circ} \mathrm{C} / \mathrm{min}$. Marina et al. [47] have observed increased interfacial resistances for ceria electrodes sintered at temperatures greater than $1100^{\circ} \mathrm{C}$, which they attribute to diffusion of $\mathrm{Ce}^{4+}$ ions into the YSZ lattice. Platinum gauze (Alfa Aesar, 52 mesh, 0.1 $\mathrm{mm}$ diameter wire) was bonded to the electrodes as a current collector by brush-painting the GDC paint (w/o pore former) onto the Pt mesh and sintering at $1200^{\circ} \mathrm{C}$. Thermocouple-grade Pt wire (TC grade, $0.4 \mathrm{~mm}$ diameter) was spot-welded to the $\mathrm{Pt}$ 
gauze as electrical leads for the polarization and impedance measurements. Figure 3-5 displays photos of a fabricated GDC cell.

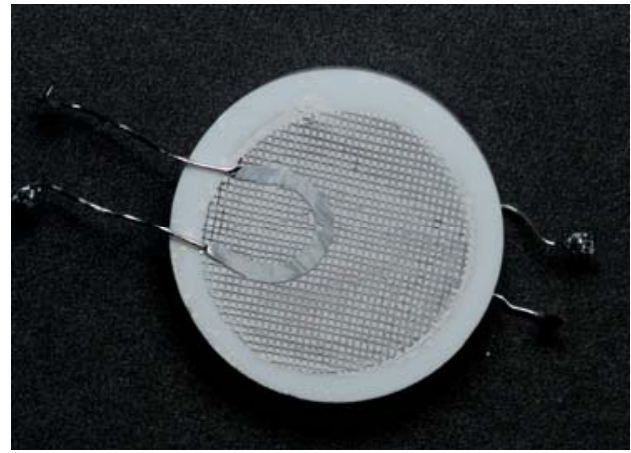

(a)

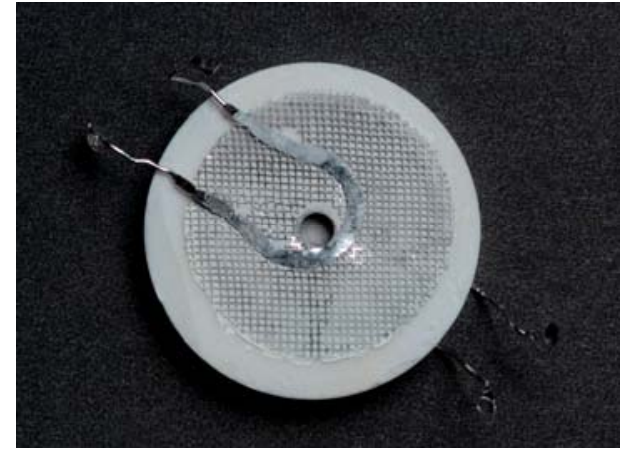

(b)

Figure 3-5. Photographs of a fabricated GDE symmetric electrode pellet cell. (a) Working electrode face and (b) counter-electrode face. Dimensions and details of this cell geometry are given in Figure 2-3.

\subsubsection{Fabrication of porous platinum electrodes}

Platinum ink (Heraeus C11-5349) was screen-printed on each face of a pellet using a $1.8 \mathrm{~cm}$ circle pattern mask identical to the one used for the GDC electrodes. The screen-painted electrodes were air-dried in an oven at $100^{\circ} \mathrm{C}$ for 20 minutes. The "green" electrodes were sintered in air for 10 minutes at $1000^{\circ} \mathrm{C}$ with a $10^{\circ} \mathrm{C} /$ min heating and cooling ramp rate. Platinum gauze was attached to the porous Pt electrode by brushpainting Pt ink (Heraeus C11-5100) on the gauze, applying the gauze to the electrodes, and firing at $1000^{\circ} \mathrm{C}$ for 10 minutes. Thermocouple-grade Pt wire (TC grade, $0.4 \mathrm{~mm}$ 
diameter) was spot-welded to the Pt gauze as electrical leads for the potential and impedance measurements.

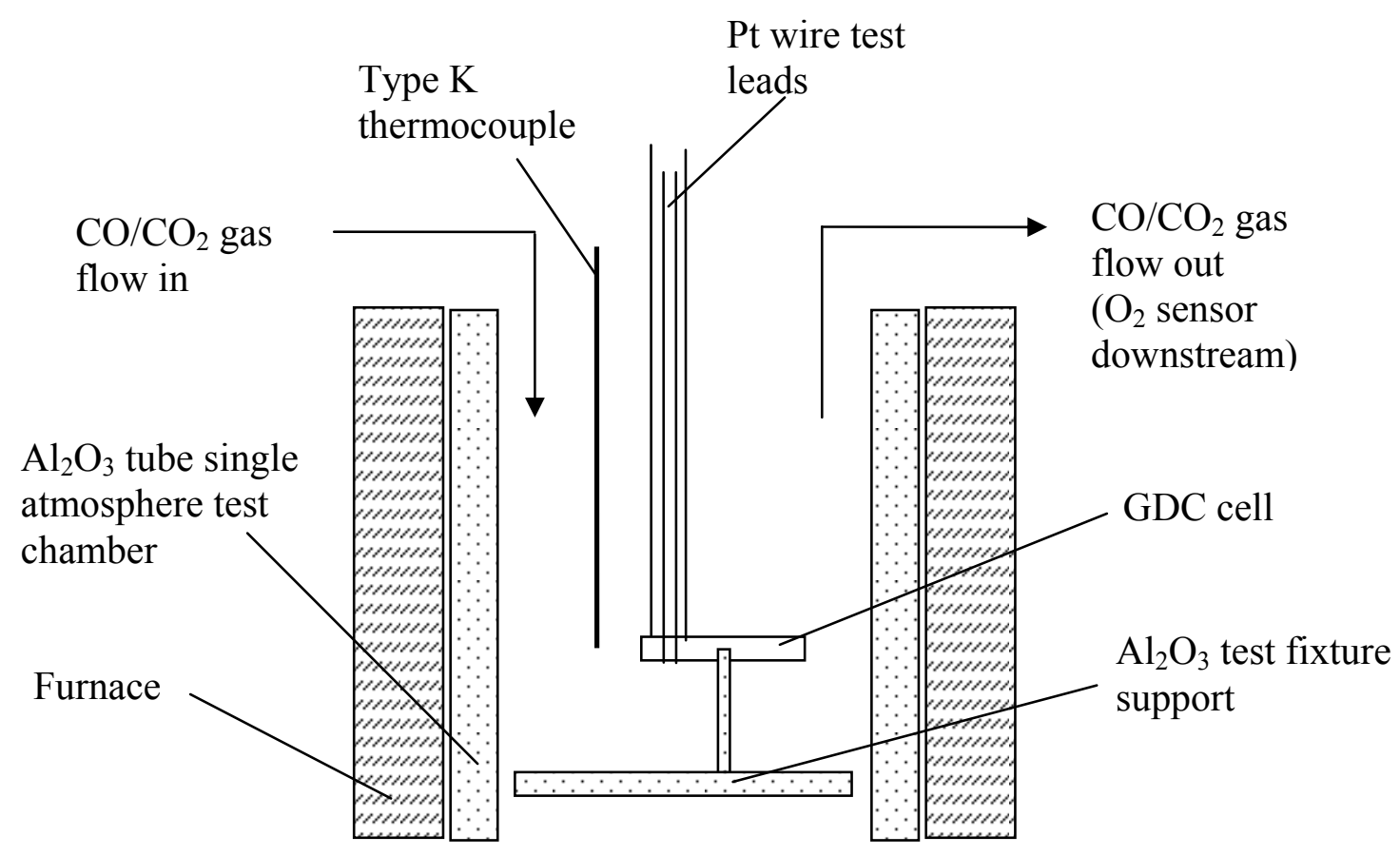

Figure 3-6. Test fixture setup for AC impedance measurements in a single atmosphere test chamber.

\subsection{Experimental setup}

\subsubsection{Test cell fixture}

The GDC cell was mounted in a test fixture, suspended on a snugly fitting zirconia tube, as shown in Figure 3-6, and installed in a 3-inch-ID, closed-one-end (COE) zirconia tube that served as a single atmosphere test chamber. The open end of the test 
chamber was sealed with a stainless steel flange (Viton ${ }^{\mathrm{TM}}$ gasket) with brazed inlet and outlet $1 / 4$ in. tubing with high temperature fittings (Conax Tech.) for the type $\mathrm{K}$ thermocouple and Pt wire lead penetrations.

\subsubsection{Gas flow system}

Figure 3-7 displays a schematic of the experimental gas flow system. The CO$\mathrm{CO}_{2}$ gas flow rate was controlled to $50-100$ standard cubic centimeters per minute (sccm) with a gas mass flow controller. The partial pressure of $\mathrm{O}_{2}\left(P_{O_{2}}\right)$ in the gas stream was monitored with a homemade Pt/YSZ/Pt oxygen sensor downstream of the test chamber, operated at $600-700^{\circ} \mathrm{C}$. Appendix B has a more detailed discussion of measurement of $P_{\mathrm{O}_{2}}$ in reducing atmospheres. As shown in Figure 3-8, the entire gas flow system, furnace, and test chamber were mounted inside a walk-in ventilated hood. The flow rate downstream of the dual-stage pressure regulator was restricted by a flow restrictor and the volumetric flow rate of the hood (2000 CFM) such that the concentration of CO would not exceed the $25 \mathrm{ppm}$ Low Exposure Level (LEL) in the hood or the lab room during normal operations. A CO sensor was installed inside the hood to monitor $\mathrm{CO}$ levels and was wired to the building central alarm system, as per NASA safety requirements, to warn building personnel if the LEL was exceeded during 24 hour/7 day unattended operations. 

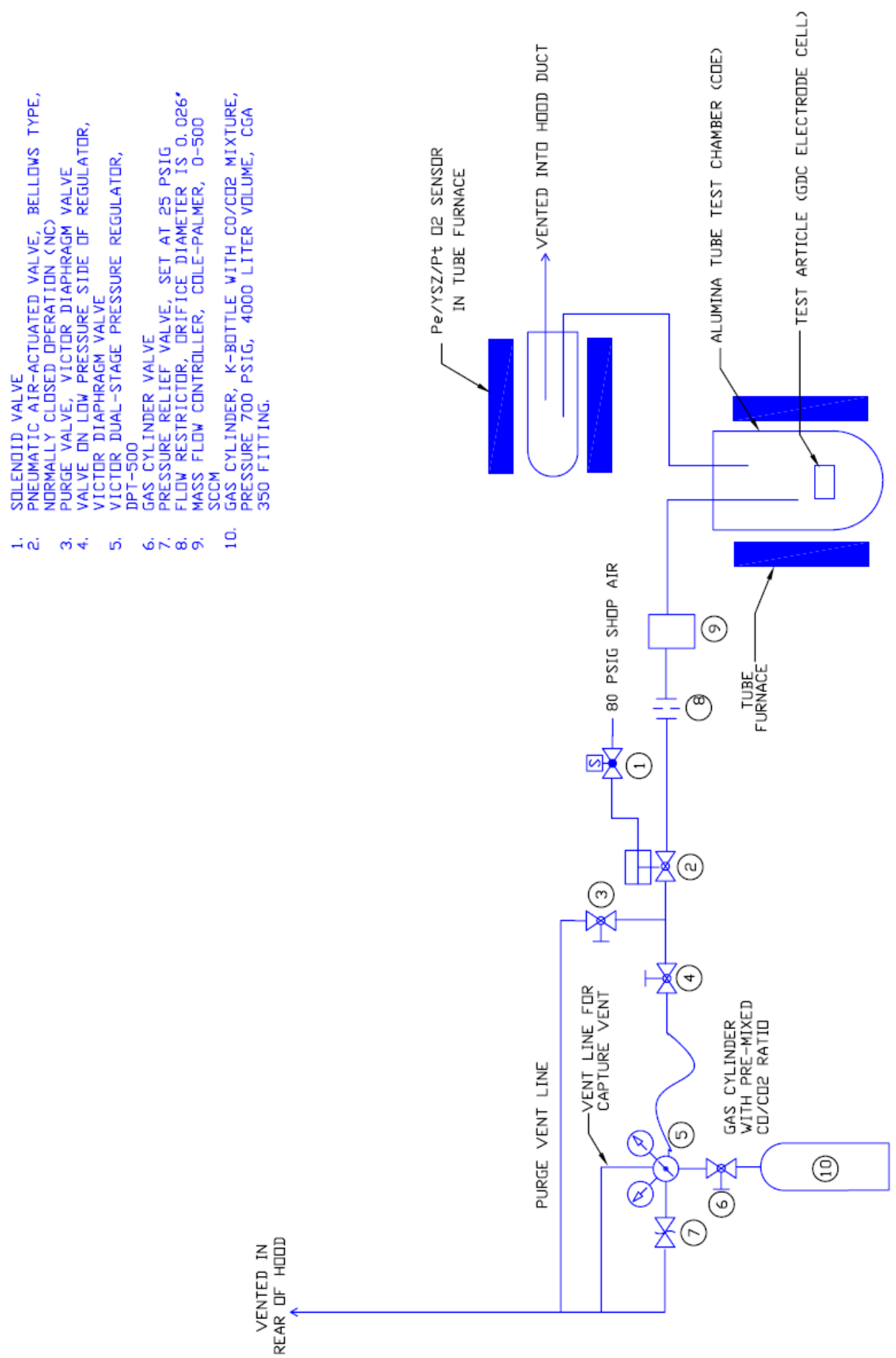

Figure 3-7. Schematic diagram of $\mathrm{CO} / \mathrm{CO}_{2}$ gas flow system. 


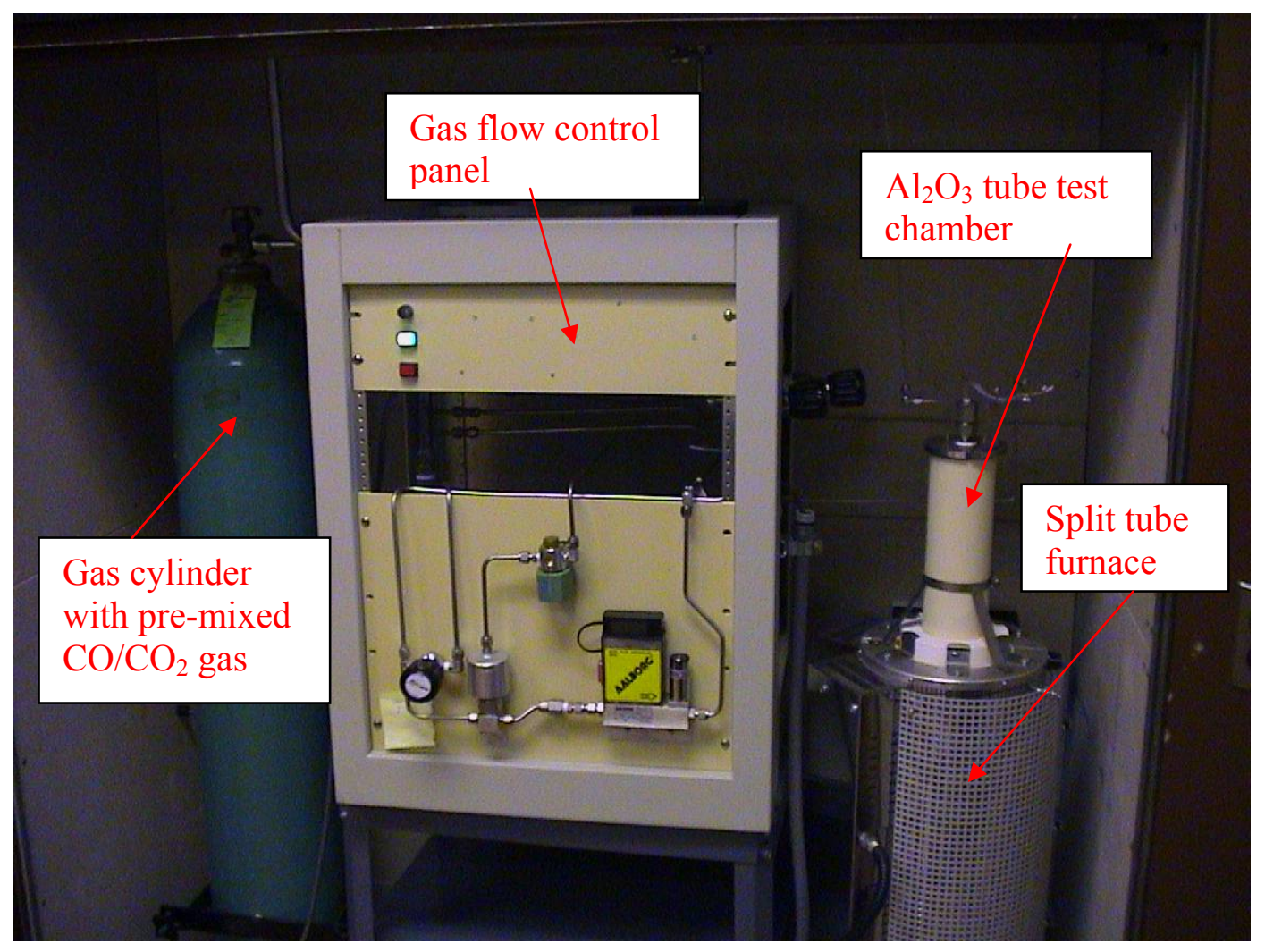

Figure 3-8. Photo of gas flow system installed in walk-in vented hood.

\subsubsection{Impedance measurements}

AC impedance measurements were made using a Solartron 1287 potentiostat and 1260 frequency spectrum analyzer under zero bias conditions; AC voltage amplitude was $10-20 \mathrm{mV}$. Measurements were made between $700^{\circ} \mathrm{C}$ and $950^{\circ} \mathrm{C}$ in gas mixtures with $\mathrm{CO} / \mathrm{CO}_{2}$ ratios of $0.05,0.25$, and 0.50 , corresponding to $P_{O_{2}}=10^{-18}-10^{-16}$ atm. The impedance measurements in this study were made across the full symmetric cell without the reference electrode. A computational analysis on the cell geometry was performed 
and showed the measurement error introduced due to the geometrical differences in the electrodes is approximately $15 \%$, compared to a true symmetric cell (see Chapter 2 ). The test matrix was cycled through three times over a period of 3 weeks of continuous operation, as a hysteresis was noted, namely the area specific resistance dropped over time of operation.

\subsubsection{Post-testing of GDC cell}

After testing, the selected cell was mounted in epoxy, cut in half and polished to examine a cross-section of the electrodes via scanning electron microscopy (SEM) and energy dispersive spectroscopy (EDS). The presence of epoxy as a mounting material has been shown not to affect EDS impurity analyses [51,54].

\subsection{Summary}

In this chapter, a rationale was provided for the choice of $40 \mathrm{~mol} \%$ gadolinia-

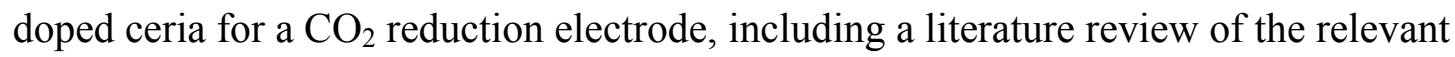
material properties of ceria and doped ceria, including a discussion of the existence of a secondary phase in this high doped oxide. Experimental details for the fabrication of the symmetric electrode pellet cells and the experimental setup were described along with several fabrication difficulties and issues. 


\section{References:}

[1] M Mogensen, T Lindegaard, UR Hansen, G Mogensen. Physical-Properties of Mixed Conductor Solid Oxide Fuel-Cell Anodes of Doped $\mathrm{CeO}_{2}$, J. Electrochem. Soc. 141 (1994) 2122-2128.

[2] BCH Steele, Materials for IT-SOFC stacks: 35 years R\&D: the inevitability of gradualness? Solid State Ionics. 134 (2000) 3-20.

[3] M Mogensen, NM Sammes, GA Tompsett. Physical, chemical, and electrochemical properties of pure and doped ceria, Solid State Ionics. 129 (2000) 63-94.

[4] A Trovarelli, Structural properties and non-stoichiometric behavior of $\mathrm{CeO}_{2}$, in: Trovarelli A (Ed.), Catalysis by Ceria and Related Materials, vol. 2, Imperial College Press, London, 2002, pp. 15-481.

[5] FA Kröger, HJ Vink, Relations between the concentrations of imperfections in crystalline solids, in: Seitz F, Turnull D (Eds.), Solid State Physics - Advances in Research and Applications, Academic Press, New York, NY, 1956, pp. 431-435.

[6] JW Dawicke, RN Blumenthal, Oxygen association pressure measurements on nonstoichiometric cerium dioxide, J. Electrochem. Soc. 133 (1986) 904-909.

[7] S Wang, H Inaba, H Tagawa. Nonstoichiometry of $\mathrm{Ce}_{0.8} \mathrm{Gd}_{0.2} \mathrm{O}_{1.9}$ - , J. Electrochem. Soc. 144 (1997) 4076-4080.

[8] S Wang, H Inaba, H Tagawa, M Dokiya, T Hashimoto. Nonstoichiometry of $\mathrm{Ce}_{0.9} \mathrm{Gd}_{0.1} \mathrm{O}_{1.95-x}$, Solid State Ionics. 107 (1998) 73-79.

[9] T Kobayashi, S Wang, M Dokiya, H Tagawa, T Hashimoto. Oxygen nonstoichiometry of $\mathrm{Ce}_{1-\mathrm{y}} \mathrm{Sm}_{\mathrm{y}} \mathrm{O}_{2-0.5 y}$ - , Solid State Ionics. 126 (1999) 349-357.

[10] B Zachau-Christianson, T Jacobsen, K West, S Skaarup, Electrochemical determination of the oxygen stoichiometry of doped ceria, in: Singhal SC, Iwahara H (Eds.), Proceedings of the Third International Symposium on Solid Oxide Fuel Cells, SOFC III, PV93-4, The Electrochemical Society, Pennington, NJ, 1993, pp. 104-111.

[11] D Perez-Coll, D Marrero-Lopez, JC Ruiz-Morales, P Nunez, Abrantes, J. C. C., JR Frade. Reducibility of $\mathrm{Ce}_{1-\mathrm{x}} \mathrm{Gd}_{\mathrm{x}} \mathrm{O}_{2 \text {-delta }}$ in prospective working conditions, J. Power Sources. 173 (2007) 291-297.

[12] P Knauth, HL Tuller. Solid-State Ionics: roots, status and future prospects, J. Am. Ceram. Soc. 85 (2002) 1654-1680. 
[13] S Dikmen, P Shuk, M Greenblatt, H Gocmez. Hydrothermal synthesis and properties of $\mathrm{Ce}_{1-\mathrm{x}} \mathrm{Gd}_{\mathrm{x}} \mathrm{O}_{2 \text {-delta }}$ solid solutions, Solid State Sciences. 4 (2002) 585-590.

[14] DK Hohnke. Ionic conduction in doped oxides with the fluorite structure, Solid State Ionics. 5 (1981) 531-534.

[15] J Faber, C Goeffroy, A Roux, A Sylvestre, P Abelard. A systematic investigation of the DC electrical conductivity of rare-earth doped ceria, Appl. Phys. A. 49 (1989) 225.

[16] JA Kilner. Fast oxygen transport in acceptor doped oxides, Solid State Ionics. 129 (2000) 13-23.

[17] S Wang, T Kobayashi, M Dokiya, T Hashimoto. Electrical and ionic conductivity of Gd-doped ceria, J. Electrochem. Soc. 147 (2000) 3606-3609.

[18] K Eguchi, T Setoguchi, T Inoue, H Arai. Electrical properties of ceria-based oxides and their application to solid oxide fuel cells, Solid State Ionics. 52 (1992) 165-172.

[19] C Kittel, Introduction to Solid State Physics, sixth ed., Wiley, New York, NY, 1986.

[20] E Ruiz-Trejo, J Maier. Electronic transport in single crystals of Gd-doped ceria, J. Electrochem. Soc. 154 (2007) B583-B587.

[21] SB Adler. Chemical expansivity of electrochemical ceramics, J Am Ceram Soc. 84 (2001) 2117-2119.

[22] G Mogensen, M Mogensen. Reduction Reactions in Doped Ceria Ceramics Studied by Dilatometry, Thermochimica Acta. 214 (1993) 47-50.

[23] M Shelef, GW Graham, RW McCabe, Ceria and other oxygen storage components in automotive catalysts, in: Trovarelli A (Ed.), Catalysis by Ceria and Related Materials, vol. 2, Imperial College Press, London, 2002, pp. 343-365.

[24] EP Murray, T Tsai, SA Barnett. A direct-methane fuel cell with a ceria-based anode, Nature. 400 (1999) 649-651.

[25] OA Marina, M Mogensen. High temperature conversion of methane on a composite gadolinia-doped ceria-gold electrode, Appl. Catal. A. 189 (1999) 117-126.

[26] H Kim, S Park, J Vohs, RJ Gorte. Direct oxidation of liquid fuels in a solid oxide fuel cell, J. Electrochem. Soc. 148 (2001) A693-A695.

[27] T Nakamura, T Kobayashi, K Yashiro, K Sato, T Kawada, J Mizusaki, Electrochemical behaviors of mixed conducting anodes for SOFC, ECS Transactions. 7 (2007) 1601-1607. 
[28] K Yashiro, S Onuma, A Kaimai, Y Nigara, T Kawada, J Mizusaki, et al. Mass transport properties of $\mathrm{Ce}_{0.9} \mathrm{Gd}_{0.1} \mathrm{O}_{2 \text {-delta }}$ at the surface and in the bulk, Solid State Ionics. 152-153 (2002) 469-476.

[29] S McIntosh, JM Vohs, RJ Gorte. An examination of lanthanide additives on the performance of Cu-YSZ cermet anodes, Electrochim. Acta. 47 (2002) 3815-3821.

[30] M Mogensen, D Lybye, K Kammer, N Bonanos, Ceria revisited: electrolyte or electrode material?, in: Singhal SC, Mizusaki, J (Eds.), Proceedings of the Ninth International Symposium on Solid Oxide Fuel Cells, SOFC IX, 2005-07, The Electrochemical Society, Pennington, NJ, 2005, pp. 1069-1074.

[31] K Kammer, M Mogensen. Oxidation of methane and hydrogen on $\mathrm{Ce}_{1-\mathrm{x}} \mathrm{Gd}_{\mathrm{x}} \mathrm{O}_{2 \text {-delta }}$ fluorites, Electrochem. Solid-State Lett. 8 (2005) A108-A109.

[32] SB Adler. Factors governing oxygen reduction in solid oxide fuel cell cathodes, Chem. Rev. 104 (2004) 4791-4843.

[33] TH Etsell, SN Flengas. Electrical properties of solid oxide electrolytes, Chem. Rev. 70 (1970) 339-376.

[34] T Kudo, H Obayashi. Oxygen ion conduction of the fluorite-type $\mathrm{Ce}_{1-\mathrm{x}} \mathrm{Ln}_{\mathrm{x}} \mathrm{O}_{2-\mathrm{x} / 2}$ ( $\mathrm{Ln}$ = Lanthanoid element), J. Electrochem. Soc. 122 (1975) 142-147.

[35] Z Tianshu, P Hing, H Huang, J Kilner. Ionic conductivity in the $\mathrm{CeO}_{2}-\mathrm{Gd}_{2} \mathrm{O}_{3}$ system $(0.05<\mathrm{Gd} / \mathrm{Ce}<0.4)$ prepared by oxalate coprecipitation, Solid State Ionics. 148 (2002) $567-573$.

[36] V Grover, AK Tyagi. Phase relations in the $\mathrm{CeO}_{2}-\mathrm{Gd}_{2} \mathrm{O}_{3}-\mathrm{ZrO}_{2}$ system, J. Solid State Chem. 177 (2004) 4197-4204.

[37] B Zachau-Christianson, T Jacobsen, S Skaarup. Electrochemical determination of oxygen stoichiometry and entropy in oxides, Solid State Ionics. 86-88 (1996) 725-731.

[38] D Perez-Coll, P Nunez, JC Ruiz-Morales, J Pena-Martinez, JR Frade. Reexamination of bulk and grain boundary conductivities of $\mathrm{Ce}_{1-\mathrm{x}} \mathrm{Gd}_{\mathrm{x}} \mathrm{O}_{2 \text {-delta }}$ ceramics, Electrochim. Acta. 52 (2007) 2001.

[39] U Hennings, R Reimert. Investigation of the structure and the redox behavior of gadolinium doped ceria to select a suitable composition as catalyst support in the steam reforming of natural gas, Appl. Catal. A-Gen. 325 (2007) 41-49.

[40] PW Atkins, Physical Chemistry, first ed., W. H. Freeman and Company, San Francisco, CA, 1978, p. 707.

[41] SJ Hong, AV Virkar. Lattice parameters and densities of rare-earth oxide doped ceria electrolytes, J. Am. Ceram. Soc. 78 (1995) 433-439. 
[42] RD Shannon. Revised effective radii and systematic studies of interatomic distances in halides and chalcogenides, Acta Crystallogr. Sec. A. 32 (1976) 751-767.

[43] Y Matsuzaki, I Yasuda. The poisoning effect of sulfur-containing impurity gas on an SOFC anode: Part I. Dependence on temperature, time, and impurity concentration, Solid State Ionics. 132 (2000) 261-269.

[44] S McIntosh, RJ Gorte. Direct hydrocarbon solid oxide fuel cells, Chem. Rev. 104 (2004) 4845-4865.

[45] M Gong, X Liu, J Trembly, C Johnson. Sulfur-tolerant anode materials for solid oxide fuel cell application, J. Power Sources. 168 (2007) 289-298.

[46] S Park, JM Vohs, RJ Gorte. Direct oxidation of hydrocarbons in a solid-oxide fuel cell, Nature. 404 (2000) 265-267.

[47] OA Marina, C Bagger, S Primdahl, M Mogensen. A solid oxide fuel cell with a gadolinia-doped ceria anode: preparation and performance, Solid State Ionics. 123 (1999) 199-208.

[48] MN Rahaman, Ceramic Processing, first ed., CRC Press, Boca Raton, FL, 2007. See pp. 284 and 293.

[49] JM Bae, Steele, B. C. H. Properties of $\mathrm{La}_{0.6} \mathrm{Sr}_{0.4} \mathrm{Co}_{0.2} \mathrm{Fe}_{0.8} \mathrm{O}_{3 \text {-delta }}$ (LSCF) double layer cathodes on gadolinium-doped cerium oxide (CGO) electrolytes - I. Role of $\mathrm{SiO}_{2}$, Solid State Ionics. 106 (1998) 247-253.

[50] KV Jensen, R Wallenberg, I Chorkendorff, M Mogensen. Effect of impurities on structural and electrochemical properties of the Ni-YSZ interface, Solid State Ionics. 160 (2003) 27-37.

[51] YL Liu, S Primdahl, M Mogensen. Effects of impurities on microstructure in Ni/YSZ-YSZ half-cells for SOFC, Solid State Ionics. 161 (2003) 1-10.

[52] RP Ingel, D Lewis. Lattice-parameters and density for $\mathrm{Y}_{2} \mathrm{O}_{3}$-stabilized $\mathrm{ZrO}_{2}$, J. Am. Ceram. Soc. 69 (1986) 325-332.

[53] LA Dunyushkina, SB Adler. Influence of electrolyte surface planarization on the performance of the porous SOFC cathodes, J. Electrochem. Soc. 152 (2005) A2040A2045.

[54] A Hauch, SH Jensen, JB Bilde-Sorensen, M Mogensen. Silica segregation in the Ni/YSZ electrode, J. Electrochem. Soc. 154 (2007) A619-A626. 


\section{CHAPTER 4}

\section{THE CO-CO $\mathrm{CO}_{2}$ EXHANGE POROUS ELECTRODE MODEL}

\subsection{Introduction}

In order to interpret and gain further insight into the impedance measurements of $\mathrm{CO}-\mathrm{CO}_{2}$ exchange on GDC electrodes, an adequate model of the kinetics and transport of the processes is required. In this chapter, a 1-D continuum-based model for $\mathrm{CO}-\mathrm{CO}_{2}$ exchange on a mixed-conducting porous electrode is derived utilized to fit the experimental impedance results presented in Chapter 5. This model was described in Green et. al. [1], and this chapter provides background and more details of the development. In addition, an alternative solution of the model for a less restrictive boundary condition is provided in Appendix E. The key assumptions and methodology of the $\mathrm{CO}-\mathrm{CO}_{2}$ exchange model are based on an oxygen reduction mixed conducting porous electrode model originally developed by Adler et. al. [2-4].

\subsubsection{Background}

It is important to assess the model type and fidelity to determine the appropriate level of modeling for the type of experimental data to be fitted to the model. Electrode level models for SOFC applications can be categorized into three types [5]: 
1. Electrode models based on mass transfer analysis - These models assume rapid reaction kinetics such that mass transfer and ohmic resistances are the limiting phenomena.

2. Macrohomogeneous porous electrode models - These electrode models do not make assumptions about one or more limiting phenomena, i.e. a step more general than the mass transfer based models, but assume the microstructure of the electrode is uniform or homogeneous.

3. Monte-Carlo or stochastic electrode models - These models are primarily used for composite electrode models and attempt to take in account the variation in the microstructure due to separate ionic and electronic conducting particles, along with the gas pores.

For this research effort, the macro-homogeneous porous electrode level of model was chosen. The first choice above was not satisfactory as it is not known whether mass transfer is limiting phenomena for our application, and since our electrode consists of a single-phase material with a microstructure that is not well-characterized, the third choice was not considered.

\subsubsection{Macro-homogeneous porous electrode models}

Newman and Tobias [6] initially provided a theoretical analyses and analytical solution for the current distribution in a porous electrode by treating the electrode as a flooded agglomerate, applicable to battery cell and flow-through cell electrodes. A number of these early models treat the electrode with a simplified microstructure. For 
example, Giner and Hunter [7] and Iczkowski and Cutlip [8] treated a Teflon ${ }^{\mathrm{TM}}$-bonded gas diffusion electrode (GDE) for alkaline fuel cells as a 1-D electrode with straight cylindrical pores. Newman and Tiedeman [9] and Newman [10] provide the general theory and formal description of the macroscopic (or macro-homogeneous) porous electrode theory and a review of flooded porous electrode applications, in particular, battery applications. Extensive work [11-14] in the polymer electrolyte membrane (PEM) fuel cell has typically resulted in more complex models without the flooded electrode assumption, in that these GDE models involve a gas, liquid, and one or several solid phases in the electrode.

In the solid oxide fuel cell (SOFC) area, Svensson et. al. $[15,16]$ developed a very detailed physical model of oxygen reduction on perovskite metal oxide cathodes, and compared with area-specific resistance (ASR) data vs. $P_{\mathrm{O}_{2}}$ for literature data on $\mathrm{La}_{1-}$ ${ }_{x} \mathrm{Sr}_{x} \mathrm{MO}_{3}$ where $(\mathrm{M}=\mathrm{Mn}, \mathrm{Co}, \mathrm{Cr}, \mathrm{Fe})$. Other researchers [17-19] have noted the importance of modeling the effect of microstructure for composite electrodes, which the macro-homogenous theory ignores. They recommend the development of micro-models, utilizing percolation theory or Monte Carlo methods to discern the effect of the ratio of ionic and electronic conducting particles in a composite electrode. Sunde [20] discusses and compares 3 modeling approaches for SOFC composite electrode models: thin film, Monte Carlo, and macroscopic (or macro-homogeneous) porous electrode methods; he concludes the Monte Carlo method is superior but the macro-homogeneous method has reasonable agreement with the Monte Carlo method in limited applicability situations, and is simpler to implement for many applied research applications. 
These modeling approaches are primarily DC or polarization type models that consist of governing and constitutive equations that assume steady-state conditions. To develop a model to interpret EIS impedance measurements adds an additional complexity due the capacitive type phenomena, which negates the steady-state assumption.

\subsubsection{Equivalent circuit models}

As impedance spectroscopy [21] began to be implemented as a measurement technique for solid state electrochemical systems, the primary technique for modeling was equivalent circuit analysis for fitting experimental impedance data and continues to be a commonly used method to date. In fact, a number of the recent $\mathrm{CO}$ oxidation works on Ni-YSZ electrodes [22-24] have utilized equivalent circuit models to fit and interpret impedance data; this more common technique has seen extensive use in modeling both aqueous and solid state electrochemical systems, including solid oxide fuel cells.

The equivalent circuit analysis method consists of constructing a model of a number of components (resistor, capacitors, etc.) in series and parallel to simulate the electrical analog of the anticipated physical processes [25]. The disadvantage of this technique is the electrical parameters may not have a direct relationship to physiochemical processes; if the model is then fitted to experimental data, the results cannot be compared to transport properties like surface exchange rate or diffusion coefficient of a chemical species. Also, this type of model does not provide any 
information about the current distribution, potential, or concentration of various species, in the electrode. Note that the reference electrode placement analysis presented in Chapter 2 of this work utilizes a very simple equivalent circuit model for the working electrode and counter-electrode boundary conditions.

\subsubsection{Continuum-based models for impedance spectroscopy applications}

For the type of electrode (mixed conductor as opposed to composite) and type of experimental data (AC impedance), a macro-homogeneous model was developed that attempts to describe impedance in terms of chemical processes (bulk diffusion and surface reaction kinetics), which can be tied directly to the properties of the mixed conductor.

To aid in solving the model, a conversion from the time domain to the frequency domain (to eliminate the time dependent derivative terms) is appropriate if the amplitude of I and V are small such that the response is linear, typical of impedance measurements. Doyle et al. [26] and Fuller [27] describe a method and assumptions for transforming the continuum-based governing equations from the time domain to the frequency domain.

This modeling methodology has also been used with moderate success on a wide variety of electrochemical systems including the rotating disk electrode [28], lithium-ion batteries [26], PEM fuel cell cathodes [14], and SOFC electrodes [4,29]. 


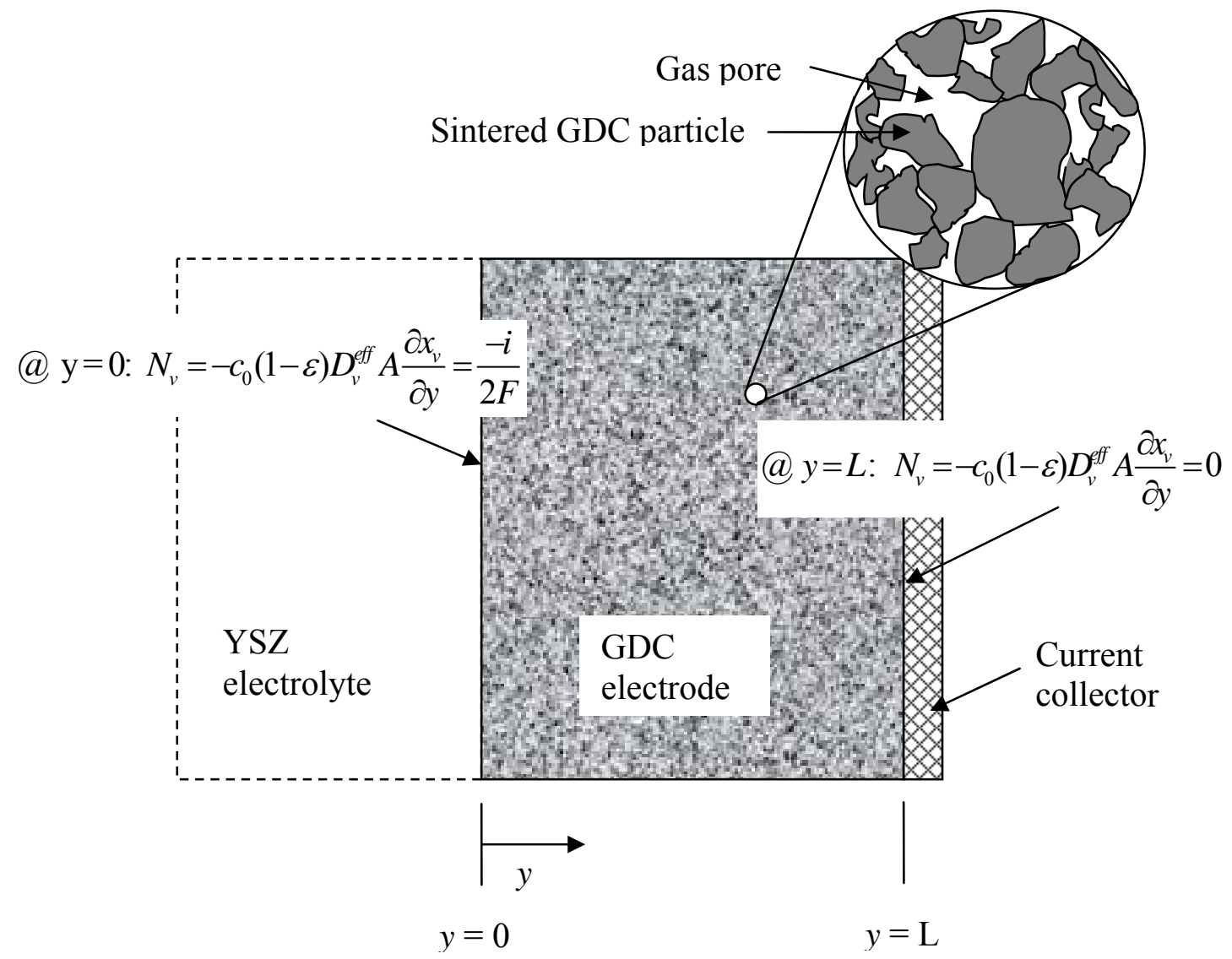

Governing equation for the model domain: $c_{0}(1-\varepsilon) \frac{\partial x_{v}}{\partial t}=c_{0}(1-\varepsilon) D_{v}^{e f f} \frac{\partial}{\partial y}\left(A \frac{\partial x_{v}}{\partial y}\right)-a r$

Figure 4-1. Geometry and model domain for 1-dimensional $\mathrm{CO}-\mathrm{CO}_{2}$ exchange porous electrode model. In the model domain, the GDC electrode is treated as a continuum consisting of two phases, the solid matrix (sintered GDC particles) and the gas phase, and each phase is assumed to co-exist at any point in the domain.

\subsection{Theory}

\subsubsection{Model domain and assumptions}

In this derivation, we develop a $\mathrm{CO}-\mathrm{CO}_{2}$ exchange one-dimensional porous electrode model closely paralleling the Adler-Lane-Steele (ALS) solid oxide cathode model $[2,4]$ for $\mathrm{O}_{2}$ reduction kinetics. The porous electrode is treated as a superposition 
of two continua: the solid matrix as a single phase mixed conductor, and the gas phase, with the various physical parameters such as porosity, surface exchange rate, vacancy diffusion coefficient volume-averaged at a particular point, ignoring the geometric detail of the electrode [30]. A representation of the 1-D model domain is given in Figure 4-1.

The following additional assumptions are made:

1. The electrode solid matrix material (GDC) is a good electronic conductor, such that the electronic transference number, $t_{e}$, is $\sim 1$ for the low $P_{\mathrm{O}_{2}}\left(=10^{-18}-10^{-16} \mathrm{~atm}\right)$ conditions encountered for the $\mathrm{CO}-\mathrm{CO}_{2}$ exchange experiments in Chapter 5 . Electronic conductivity measurements of 0.13 $\mathrm{S} / \mathrm{cm}[31]$ on $40 \mathrm{~mol} \% \mathrm{GDC}$ at $1000^{\circ} \mathrm{C}$ under highly reduced conditions $\left(P_{\mathrm{O}_{2}}=10^{-18} \mathrm{~atm}\right)$ support this assumption. In addition, calculations of the free electron density in GDC from thermogravimetry measurements is on the order of $10^{21} \mathrm{~cm}^{-3}$ [32], which is an order of magnitude lower than a highly conductive metal like $\mathrm{Cu}$ at $8.52 \times 10^{22} \mathrm{~cm}^{-3}$ [33]. Dikmen et al. [34] reported the transference number, $t_{i}>0.95$ for GDC for samples with $x=0.0-0.30$, by measuring the EMF in an oxygen concentration cell and comparing with the theoretical Nernst potential.

2. The oxygen vacancy diffusion in the solid matrix is bulk (not surface diffusion) dominated. Values for surface diffusion are sparse in the literature, at least for solid oxide materials. Boukamp [35] gives an estimate of $1 \times 10^{-6}$ 
$\mathrm{cm}^{2} / \mathrm{s}$ on Ti-doped YSZ/Ni anodes in $\mathrm{H}_{2}-\mathrm{H}_{2} \mathrm{O}$, implying surface diffusion is on the same order as bulk diffusion.

3. The impedances due to charge transfer across the current collector-electrode interface and ionic transport across the electrode-electrolyte interface are small and can be neglected. It is anticipated that if these impedances are substantial in magnitude, they are ohmic in nature and indistinguishable from the electrolyte resistance in our experimental measurements.

4. Gas phase diffusion effects are not limiting. Measurements were performed with a zero bias (i.e. open circuit potential condition) with a small AC potential perturbation amplitude of $10-15 \mathrm{mV}$, minimizing $\mathrm{CO}-\mathrm{CO}_{2}$ exchange rates and thus variation in the gas concentration in the porous electrode. Primdahl and Mogensen [36,37] have reported on gas conversion impedance effects in SOFC anodes but have demonstrated this phenomena does not occur for symmetric cells tested in a common atmosphere [38]. Adler et. al. [2] showed gas diffusion resistance effects can be significant in oxygen reduction electrodes on symmetric cells, but at $P_{\mathrm{O}_{2}}$ values below $0.07 \mathrm{~atm}$.

5. The bulk of the carbon dioxide exchange reaction occurs on the inner electrode surface within the volume of the electrode, and can be neglected at the outer (or current collector) electrode surface at $y=L$. Appendix E provides a rationale for this assumption. 


\subsubsection{Governing equations for the solid matrix}

Because of assumption \#4 in the previous section, the gas concentration gradients in the porous electrode can be neglected, and the GDC solid matrix need only be considered. The governing equations for the GDC solid matrix are [10]:

$$
\mathbf{N}_{i}=-\frac{D_{i} c_{i}}{R T} \nabla \mu_{i}+c_{i} \mathbf{v}
$$

where $i=$ species, i.e. electrons, holes, or oxygen vacancies

$$
\begin{aligned}
\mathbf{N}_{i} & =\text { flux of component } i \text { in } \frac{\text { moles }}{\mathrm{cm}^{2} \cdot \mathrm{s}} \\
D_{i} & =\text { diffusion coefficient of component } i \text {, in } \frac{\mathrm{cm}^{2}}{\mathrm{~s}} \\
c_{i} & =\text { concentration of } i, \text { in } \frac{\mathrm{mol}}{\mathrm{cm}^{2}} \\
\mu_{i} & =\text { electrochemical potential of component } i, \text { in } \frac{\mathrm{J}}{\mathrm{mol}} \\
\mathbf{v} & =\text { fluid velocity of component } i, \text { in } \frac{\mathrm{cm}}{\mathrm{s}} \\
& =0 \text { (transport in a solid state medium) }
\end{aligned}
$$

The equation of continuity for species $i$ is:

$$
\frac{\partial c_{i}}{\partial t}=-\nabla \cdot N_{i}+R_{i}
$$

where $\mathrm{R}_{i}=$ the rate of generation of component $i$, in $\frac{\mathrm{mol}}{\mathrm{cm}^{2} \cdot \mathrm{s}}$

The conservation of charge in the solid matrix is expressed as:

$$
0=B+\sum_{i=1}^{n} Z_{i} C_{i}
$$

where $B=$ background charge density, in $\frac{\mathrm{mol}}{\mathrm{cm}^{3}}$

$Z_{i}=$ charge of component $i$

$n=$ total number of components $=3$ 
Finally, the current density at any point is the summation of the fluxes of all charged species:

$$
\mathbf{i}=F \sum_{i=1}^{n} z_{i} \mathbf{N}_{i}
$$

\subsubsection{Speciation in the mixed conductor}

The GDC solid matrix is treated as a moderately dilute solid solution consisting of three species: oxygen vacancies, electrons, and holes. In actuality, there are five species in the GDC solid matrix: $C e_{C e}^{x}, C e_{C e}^{\prime}, G d_{C e}^{\prime}, O_{O}^{x}$, and $V_{o}^{\bullet}$, but the $G d$ and $C e$ cations are indistinguishable [2] and as such, can be represented by holes and electrons. The oxygen vacancies and oxygen anions are not independent quantities due to site conservation, and as such, oxygen vacancies are chosen as the composition variable [3]. This reduces the number of species to holes, electrons, and oxygen vacancies. As described in Chapter 3, reduced ceria is n-type conductive, and the hole concentration will be small compared to the electron concentration [32], and therefore, is neglected. With assumption \#1, the gradient of the electrochemical potential of electrons is neglected, so that the model is reduced to ionic phase transport in the solid matrix of the electrode. The onedimensional equation of conservation for our chosen species, oxygen vacancies, combined with solid state diffusion (i.e. Fick's first law) becomes:

$$
c_{0}(1-\varepsilon) \frac{\partial x_{v}}{\partial t}=c_{0}(1-\varepsilon) D_{v}^{\text {eff }} \frac{\partial}{\partial y}\left(A \frac{\partial x_{v}}{\partial y}\right)-a r
$$


where $x_{v}=$ mole fraction of vacancies in the GDC electrode solid matrix

$c_{o}=$ concentration of oxygen lattice sites in GDC

$\varepsilon=$ the electrode porosity

$D_{v}^{e f f}=\frac{D_{v}}{\tau_{s}}=$ the effective vacancy diffusion coefficient

$\tau_{\mathrm{s}}=$ tortuosity of the solid matrix of the electrode

$a=$ surface area per unit volume of the electrode

$r=$ rate of the $\mathrm{CO}_{2}$ reduction reaction per unit surface area of the electrode

The $A$ parameter in Equation (4-5) is referred to as the thermodynamic factor (or alternatively, the thermodynamic enhancement factor) [39] and is defined in this work as:

$$
A=-\frac{1}{2} \frac{\partial P_{O_{2}}}{\partial x_{v}^{e q b}}
$$

where $x_{v}^{e q b}=$ vacancy concentration at equilibrium

Values of $A$ for solid oxide materials can be calculated from a defect model based on oxygen non-stoichiometry measurements. Appendix D provides a derivation of the defect model used to estimate $A$ for GDC in this work.

\subsubsection{The $\mathrm{CO}-\mathrm{CO}_{2}$ exchange reaction rate at the mixed conductor electrode surface}

The CO-CO $\mathrm{CO}_{2}$ exchange reaction occurring over the surface of the porous electrode, using Kröger-Vink notation [40] is:

$\mathrm{CO}_{2}+\mathrm{V}_{\mathrm{O}}^{\bullet \bullet}+2 e^{-}=\mathrm{CO}+\mathrm{O}_{\mathrm{O}}^{x}$ 
To describe the rate of $\mathrm{CO}-\mathrm{CO}_{2}$ exchange, a general non-equilibrium reaction rate expression is applied:

$r=\Re_{0}\left(1-e^{-\frac{\Lambda}{2 R T}}\right)=\Re_{0}\left[1-\left(\frac{P_{O_{2}}}{f_{O_{2}}^{\text {solid }}}\right)^{-\frac{1}{2}}\right]$

where $\Lambda=R T \ln \frac{P_{\mathrm{O}_{2}}}{f_{\mathrm{O}_{2}}^{\text {solid }}}=$ a thermodynamic driving force

$f_{\mathrm{O}_{2}}^{\text {solid }}=$ the oxygen fugacity in the bulk GDC solid matrix

$\mathfrak{R}_{0}=$ the exchange rate coefficient

Appendix $\mathrm{F}$ provides further details on the form and assumptions used in derivation of this non-linear rate expression. Equation (4-8) is linearized, assuming a small perturbation of $x_{v}$ from the equilibrium vacancy mole fraction, $x_{v}^{\text {eqb }}$, and expanded about this value using a Taylor series:

$r=\Re_{0}[\underbrace{\left.1-\left(\frac{P_{O_{2}}}{f_{O_{2}}^{\text {solid }}}\right)^{-\frac{1}{2}}\right]_{x_{v}=x_{v}^{e q b}}}_{=0}+\Re_{0}\left[\frac{1}{2}(\underbrace{\left.\frac{P_{O_{2}}}{f_{O_{2}}^{\text {solid }}}\right)^{-\frac{3}{2}}}_{=1} \frac{\partial}{\partial x_{v}}\left(\frac{P_{O_{2}}}{f_{O_{2}}^{\text {solid }}}\right)]_{x_{v}=x_{v}^{e q b}}\left(x_{v}-x_{v}^{e q b}\right)+\cdots\right.$

neglecting the second order and higher terms in the series and noting that by definition, $f_{\mathrm{O}_{2}}^{\text {solid }}=P_{\mathrm{O}_{2}}$ at $x_{v}=x_{v}^{\text {eqb }}$. 
The portion of the second term in brackets in equation (4-9) is expanded using the chain rule:

$$
\begin{aligned}
& \left.\frac{1}{2} \frac{\partial}{\partial x_{v}}\left(\frac{P_{O_{2}}}{f_{O_{2}}^{\text {solid }}}\right)\right|_{x_{v}=x_{v}^{e q b}}=\left.\frac{\partial \frac{P_{O_{2}}}{f_{O_{2}}^{\text {solid }}}}{\partial \ln \frac{P_{O_{2}}}{f_{O_{2}}^{\text {solid }}}}\left(-\frac{1}{2}\right) \frac{\partial \ln \frac{f_{O_{2}}^{\text {solid }}}{P_{O_{2}}}}{\partial \ln x_{v}} \frac{\partial \ln x_{v}}{\partial x_{v}}\right|_{x_{v}=x_{v}^{e q b}} \\
& =\underbrace{\frac{P_{O_{2}}}{f_{O_{2}}^{\text {solid }}}}_{=1}(\underbrace{-\frac{1}{2} \frac{\partial \ln f_{O_{2}}^{\text {solid }}}{\partial \ln x_{v}}}_{=A}+\underbrace{\frac{1}{2} \frac{\partial \ln P_{O_{2}}}{\partial \ln x_{v}}}_{=0}) \underbrace{\frac{1}{x_{v}}}_{=\frac{1}{x_{v}^{\text {eqb }}}}
\end{aligned}
$$

Note that the second term in the brackets, $\frac{1}{2} \frac{\partial \ln P_{\mathrm{O}_{2}}}{\partial \ln x_{v}}$, is zero since $P_{\mathrm{O}_{2}}$ is constant in the gas phase of the electrode due to the assumption of no gas concentration gradients (Assumption \#4).

Substituting the results of this expansion back into Equation (4-9) reduces Equation (4-8) to the following linearized form:

$r=A \Re_{0}\left(\frac{x_{v}-x_{v}^{e q b}}{x_{v}^{e q b}}\right)$

\subsubsection{Solution for the vacancy concentration in the electrode}

The following boundary conditions are applied to the model. At the electrode/electrolyte interface, the vacancy flux, $N_{v}$, is equal to the applied current: 
@ $\mathrm{y}=0: N_{v}=-c_{0}(1-\varepsilon) D_{v}^{e f f} A \frac{\partial x_{v}}{\partial y}=\frac{-i}{2 F}$

At the outer surface of the electrode, the vacancy flux must equal the reaction rate, or

@ $y=L: N_{v}=-c_{0}(1-\varepsilon) D_{v}^{e f f} A \frac{\partial x_{v}}{\partial y}=(1-\varepsilon) r$

But for this model, the reaction rate at this surface is assumed to be negligible, by Assumption \#5 in section 4.2.2:

@ $y=L: N_{v}=0 \Rightarrow \frac{\partial x_{v}}{\partial y}=0$

As stated, Assumption \#5 implies the bulk of the reaction occurs on the much greater internal surface area of the electrode, compared to the external electrode surface at the current collector interface.

Equation (4-5) is non-dimensionalized using the dimensionless variables defined in Table 4-1 to obtain:

$\frac{\partial \psi}{\partial \tau}=\frac{\partial^{2} \psi}{\partial \xi^{2}}-\psi$ 


\begin{tabular}{|c|c|c|}
\hline Non-dimensional variable & Units & Description \\
\hline$l_{\delta}=\sqrt{\frac{(1-\varepsilon) x_{v}^{e q b} c_{0} D_{v}^{e f f}}{a \Re_{0}}}$ & $\mathrm{~cm}$ & $\begin{array}{l}\text { Utilization thickness of } \\
\text { electrode. }\end{array}$ \\
\hline$R_{\text {chem }}=\frac{R T}{2 F^{2}} \sqrt{\frac{\tau}{(1-\varepsilon) c_{v} D_{v} a \Re_{0}}}$ & $\Omega-\mathrm{cm}^{2}$ & $\begin{array}{l}\text { Characteristic resistance } \\
\text { of electrode due to } \\
\text { chemical processes. }\end{array}$ \\
\hline$t_{c h e m}=\frac{C_{v}(1-\varepsilon)}{A a \Re_{0}}$ & $\mathrm{~S}$ & $\begin{array}{l}\text { Characteristic time } \\
\text { constant of electrode. }\end{array}$ \\
\hline$\psi=\frac{x_{v}-x_{v}^{e q b}}{x_{v}^{e q b}}$ & dimensionless & $\begin{array}{l}\text { Dimensionless vacancy } \\
\text { mole fraction scaled to } \\
\text { the vacancy mole fraction } \\
\text { at equilibrium, } x_{v}^{e q b} \text {. }\end{array}$ \\
\hline$\xi=\frac{y}{l_{\delta}}$ & dimensionless & $\begin{array}{l}\text { Dimensionless length } \\
\text { (thickness of electrode) } \\
\text { scaled to the utilization } \\
\text { thickness, } l_{\delta} \text {. }\end{array}$ \\
\hline $\begin{aligned} \tau & =\frac{t}{t_{\text {chem }}} \\
\sigma & =\omega t_{\text {chem }}\end{aligned}$ & dimensionless & $\begin{array}{l}\text { Dimensionless time and } \\
\text { frequency scaled to the } \\
\text { characteristic time } \\
\text { constant, } t_{\text {chem }} \text {. }\end{array}$ \\
\hline$\phi=\frac{L}{l_{\delta}}$ & dimensionless & $\begin{array}{l}\text { Ratio to scale thickness of } \\
\text { electrode to utilization } \\
\text { thickness, } l_{\delta} .\end{array}$ \\
\hline$\alpha=\frac{\tilde{i}}{2 F A} \sqrt{\frac{1}{a \Re_{0}(1-\varepsilon) x_{v}^{e q b} c_{0} D_{v}^{e f f}}}$ & dimensionless & $\begin{array}{l}\text { Dimensionless current } \\
\text { density scaled to current } \\
\text { density perturbation } \\
\text { amplitude }\end{array}$ \\
\hline
\end{tabular}

Table 4-1. Characteristic and dimensionless variables for derivation of $\mathrm{CO}-\mathrm{CO}_{2}$ exchange model. 
Equation (4-15) is transformed from time space to frequency space by assuming the vacancy concentration will respond in a sinusoidal manner and this response is separable, as is commonly done in continuum-based impedance models [26]:

$\psi(\xi, \tau)=\tilde{\psi}(\xi) e^{j \sigma \tau}$

where $\tilde{\psi}=$ dimensionless vacancy mole fraction as function of position, $\xi$, alone $j=\sqrt{-1}$

Substituting Equation (4-16) into (4-15), taking the time derivative, and simplifying, the governing equation becomes an ordinary differential equation (ODE) in the dimensionless length variable, $\xi$ :

$$
\frac{d^{2} \tilde{\psi}}{d \xi^{2}}=(1+j \sigma) \tilde{\psi}
$$

Similarly, the two boundary conditions are converted to non-dimensional form:

$$
\begin{aligned}
& \text { @ } \xi=0: \frac{d \tilde{\psi}}{d \xi}=\alpha \\
& \text { @ } \xi=\phi: \frac{d \tilde{\psi}}{d \xi}=0
\end{aligned}
$$




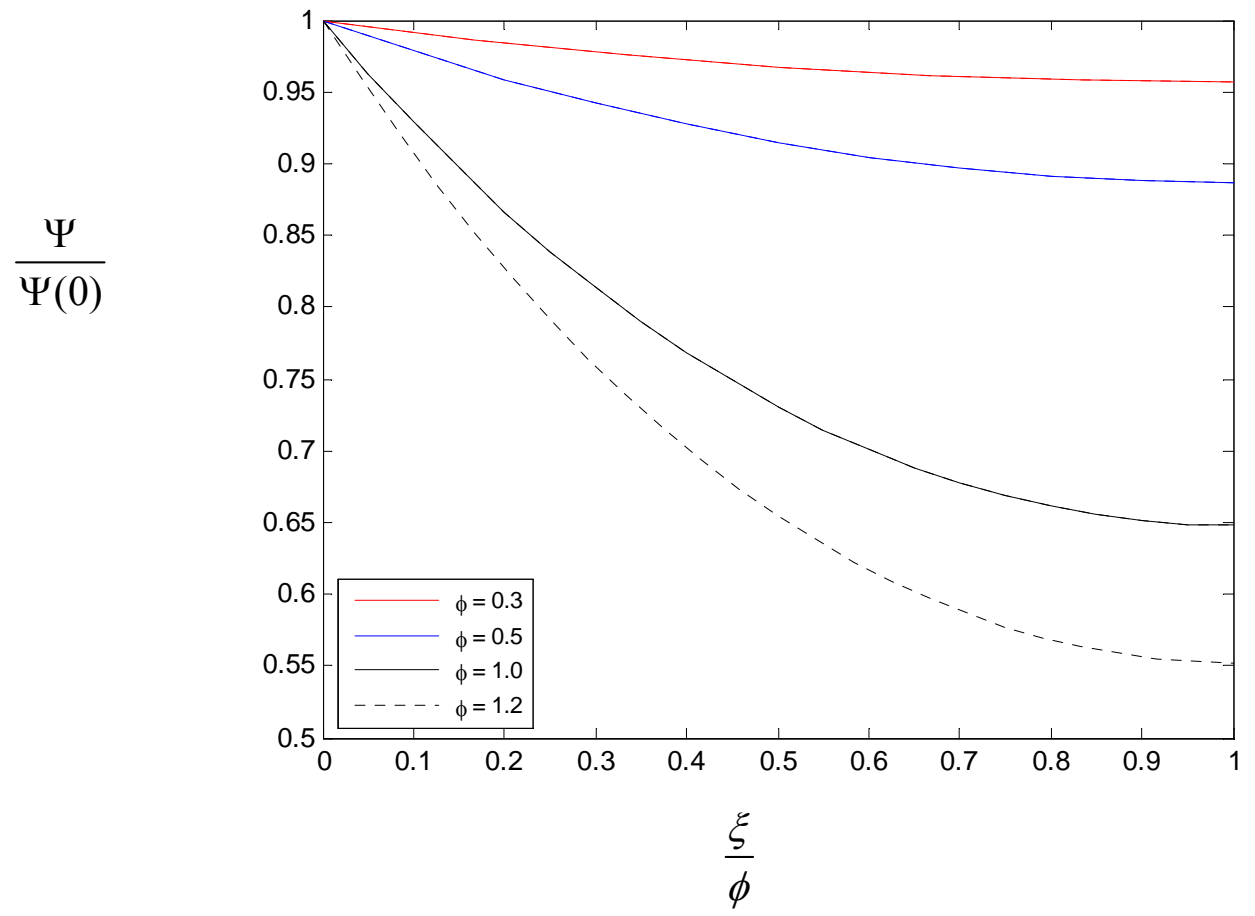

Figure 4-2 Dimensionless vacancy concentration vs. electrode thickness for several values of the utilization thickness ratio, $\phi$. Solution to Equation (4-21) for $\sigma=0$ (i.e. $\omega=0)$.

Equation (4-17) can be solved using separation of variables and has the following general solution:

$\tilde{\psi}=c_{1} \cosh (\sqrt{1+j \sigma} \xi)+c_{2} \sinh (\sqrt{1+j \sigma} \xi)$

where $c_{1}, c_{2}$ are integration constants. 
Applying the boundary conditions given in equations (4-18) and (4-19), the following explicit closed form solution is obtained:

$$
\tilde{\psi}=\frac{\alpha}{\sqrt{1+j \sigma}} \frac{\cosh [\sqrt{1+j \sigma}(\phi-\xi)]}{\sinh [\sqrt{1+j \sigma} \phi]}
$$

Figure 4-2 displays the steady-state (i.e. $\sigma=0$ ) solution to Equation (4-21) for several values of the utilization thickness, $\phi$. For $\phi \geq 1.0$, the utilization thickness is less than the electrode thickness, $l_{\delta} \leq L$, and from the vacancy concentration profile, indicates that neither kinetics nor vacancy diffusion are limiting. For values of $\phi$ less than 1.0, or $l_{\delta} \geq L$, the electrode thickness is fully utilized and the vacancy concentration profile levels off, implying kinetics, rather than vacancy diffusion, is the limiting factor. Note that for values of $\phi<1.0$, the boundary condition at $y=L$ is no longer zero, but the model is still valid in this regime. Because of the dimensionless form, the Equation (4-19) boundary condition scales with $\phi$, allowing the boundary condition to be met for this more generalized case. A quantitative rationale for the zero vacancy flux assumption at $y=L$ (even for $l_{\delta}>L$ ) is discussed in Appendix E. 


\subsubsection{Potential (or voltage) response to the current perturbation}

In order to calculate impedance, $Z_{\text {chem }}$, the voltage response to the current perturbation is required. The voltage of the ionic phase can be described by the Nerst potential:

$V=\frac{R T}{4 F} \ln \left(\frac{f_{O_{2}}^{\text {solid }}}{P_{\mathrm{O}_{2}}}\right)$

For small current perturbations, the voltage response to be small and in the linear regime; in order to describe in terms of $x_{v}$, Equation (4-22) is linearized by expanding about $x_{v}^{\text {eqb }}$ using a Taylor series, i.e. similar to our development for the $\mathrm{CO}_{2}$ exchange rate:

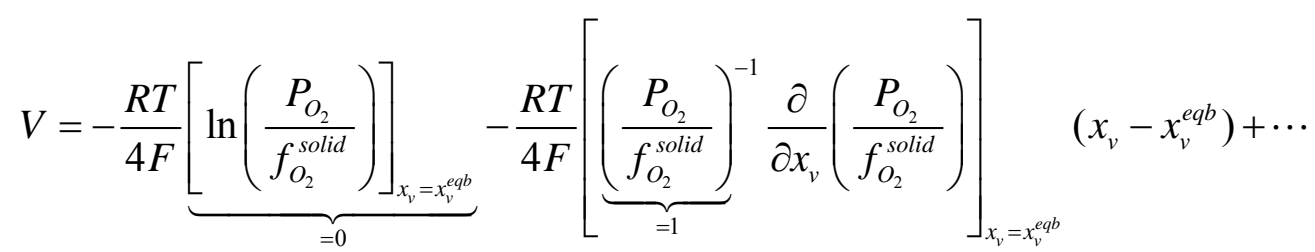

The resulting relationship for the voltage response relationship is, in terms of the dependent variable, $\tilde{\psi}$ :

$V=-\frac{R T}{4 F} A\left(\frac{x_{v}-x_{v}^{e q b}}{x_{v}^{e q b}}\right)=-\frac{R T}{4 F} A \psi=-\frac{R T}{4 F} A \tilde{\psi} e^{j \sigma \tau}$ 


\subsubsection{Relationship for $Z_{\text {chem }}$, the impedance due to chemical processes}

Finally, the "chemical" impedance is determined by evaluating the voltage, V, and current density, $i$, at $\xi=0$ (for convenience):

$$
\begin{aligned}
Z_{\text {chem }} & =\left.\frac{V}{i}\right|_{\xi=0}=\left.\frac{-\frac{R T}{4 F} A \tilde{\psi} e^{j \sigma \tau}}{2 F A \sqrt{a \Re_{0}(1-\varepsilon) x_{v}^{e q b} c_{0} D_{v}^{e f f}} \frac{\partial \tilde{\psi}}{\partial \xi} e^{j \sigma \tau}}\right|_{\xi=0} \\
& =\frac{R_{\text {chem }}}{\sqrt{1+j \sigma} \tanh (\phi \sqrt{1+j \sigma})}
\end{aligned}
$$

Substituting $\sigma=\omega t_{\text {chem }}$ and we get the following equation:

$$
Z_{\text {chem }}=\frac{R_{\text {chem }}}{\sqrt{1+j \omega t_{\text {chem }}} \tanh \left(\phi \sqrt{1+j \omega t_{\text {chem }}}\right)}
$$

$R_{\text {chem }}$ and $t_{\text {chem }}$ are a characteristic resistance and a characteristic time constant respectively; both are defined in Table 4-1 in terms of the material properties.

\subsection{Discussion}

Equation (4-26) is the model equation that will be utilized in Chapter 5 to fit measured AC impedance data and extract kinetic and transport properties for the GDC electrode. This result is similar to the one obtained for the Adler-Lane-Steele (ALS) solid oxide cathode model $[2,4]$ for $\mathrm{O}_{2}$ exchange kinetics. This similarity is primarily due to linearization of the rate equation, which essentially implies the rate is first order in 
vacancy mole fraction for both $\mathrm{CO}-\mathrm{CO}_{2}$ and $\mathrm{O}_{2}$ exchange for small perturbations from equilibrium.

The essence of this model is that it can distinguish between kinetically limited versus co-limited behavior. Figure 4-3 displays Nyquist plots for some sample solutions to Equation (4-26) for varying values of $\phi$. For large values of $\phi$ the utilization thickness, $l_{\delta}$, is small and $\tanh (\phi) \rightarrow 1$ in the denominator of equation (4-26), reducing the model to two parameters, approximating a Gerischer response [41]:

$$
Z_{\text {chem }}=\frac{R_{\text {chem }}}{\sqrt{1+j \omega t_{\text {chem }}}}
$$

This "half tear-drop" shaped response is observed in a number of solid-state electrochemical systems $[42,43]$ and indicates that both kinetics and diffusion are limiting [44]; this condition is referred to as "co-limiting." For smaller values of $\phi$, the utilization thickness, $l_{\delta}$, gets larger and the response of $Z_{c h e m}$ has a distinctive abrupt change to a smaller linear arc at high frequencies, and a large nearly circular arc for the mid and low frequencies. Inspecting the relationship for $l_{\delta}$ in Table 4-1, shows that a large $l_{\delta}$ corresponds to $D_{v}^{\text {eff }}>\mathfrak{R}_{0}$, indicating diffusion is faster than the kinetics and is referred to as "kinetics-limiting"; specifically, this condition occurs for $\phi<0.3$. 

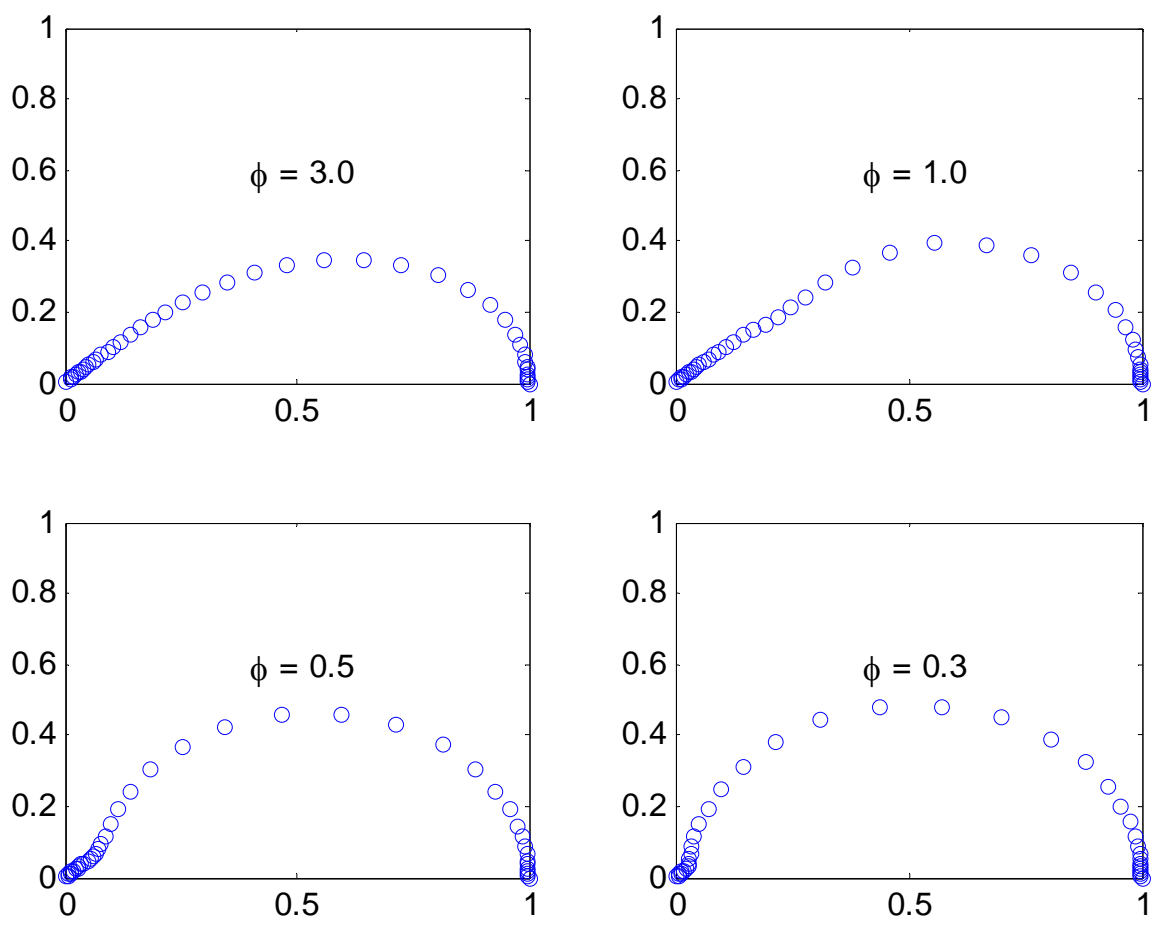

Figure 4-3. Examples of different responses of derived $\mathrm{CO}-\mathrm{CO}_{2}$ exchange model for different values of $\phi$, the utilization thickness ratio. Nyquist plots of spectra predicted by Equation (4-26). Ordinate of each plot is $-\operatorname{Im}\left[Z_{c h e m}\right]$ and abscissa is $\operatorname{Re}\left[Z_{\text {chem }}\right]$.

$\mathrm{AC}$ impedance measurement is by definition, a linearized measurement technique [45]; because of this implicit assumption, the $\mathrm{CO}-\mathrm{CO}_{2}$ exchange reaction mechanism cannot be determined using this technique [46]. Recent developments in non-linear AC impedance spectroscopy [47] may potentially offer a means of inferring the mechanism for these and similar electrochemical reactions by measuring and evaluating the nonlinear impedance response, i.e. the second and higher order harmonics of the impedance spectra. 


\subsection{Summary}

In this chapter, a mathematical model for $\mathrm{CO}-\mathrm{CO}_{2}$ exchange on a mixed conducting electrode was derived using porous electrode theory. The model is expressed in terms of three parameters, $R_{c h e m}$, a characteristic resistance, $t_{c h e m}$, a characteristic time constant, and $\phi$, a measure of the thickness of the electrode that is kinetically active. These three parameters can be related to measurable physiochemical kinetic, transport, and thermodynamic parameters via the relationships in Table 4-1. Values for these parameters, specifically: the exchange rate, $\mathfrak{R}_{0}$, and the vacancy diffusion coefficient, $D_{v}$, can thus be extracted from impedance data by fitting to Equation (4-26), and comparing to values for these parameters measured utilizing other techniques, as a way of assessing the validity of the model. In Chapter 5, this methodology is implemented on experimental AC impedance data measured on GDC electrode in $\mathrm{CO}-\mathrm{CO}_{2}$ atmospheres.

\section{References:}

[1] RD Green, CC Liu, SB Adler. Carbon dioxide reduction on gadolinia-doped ceria cathodes, Solid State Ionics. 179 (2008) 647-660.

[2] SB Adler, JA Lane, BCH Steele. Electrode kinetics of porous mixed-conducting oxygen electrodes, J.Electrochem.Soc. 143 (1996) 3554-3564.

[3] SB Adler, JA Lane, BCH Steele. Fundamental Issues in Modeling of MixedConductors, A rebuttal to comments on "Electrode Kinetics of Porous Mixed-Conducting Oxygen Electrodes", J.Electrochem.Soc. 144 (1997) 1884-1890.

[4] SB Adler. Mechanism and kinetics of oxygen reduction on porous $\mathrm{La}_{1-\mathrm{x}} \mathrm{Sr}_{\mathrm{x}} \mathrm{CoO}_{3 \text {-delta }}$ electrodes, Solid State Ionics. 111 (1998) 125-134. 
[5] MA Khaleel, JR Selman, Cell, Stack, and System Modeling, in: Singhal SC, Kendall K (Eds.), High Temperature Solis Oxide Fuel Cells: Fundamentals, Design, and Applications, first ed., Elsevier Ltd., Kidlington, Oxford, UK, 2003, pp. 405-322.

[6] JS Newman, CW Tobias. Theoretical analysis of current distribution in porous electrodes, J.Electrochem.Soc. 109 (1962) 1183-1191.

[7] J Giner, C Hunter. The mechanism of operation of the Teflon-Bonded gas diffusion electrode: a mathematical model, J.Electrochem.Soc. 116 (1969) 1124-1130.

[8] RP Iczkowski, MB Cutlip. Voltage losses in fuel cell cathodes, J. Electrochem. Soc. 127 (1980) 1433-1440.

[9] J Newman, W Tiedemann. Porous electrode theory with battery applications, AICHE J. 21 (1975) 25-41.

[10] J Newman, KE Thomas-Alyea, Electrochemical Systems, third ed., WileyInterscience, Hoboken, NJ, 2004.

[11] DM Bernardi, MW Verbrugge. Mathematical model of a gas diffusion electrode bonded to a polymer electrolyte, AICHE J. 37 (1991) 1151-1163.

[12] TE Springer, TA Zawodzinski, S Gottesfeld. Polymer electrolyte fuel cell model, J. Electrochem. Soc. 138 (1991) 2334-2342.

[13] ML Perry, J Newman, EJ Cairns. Mass transport in gas-diffusion electrodes: a diagnostic tool for fuel-cell cathodes, J. Electrochem. Soc. 145 (1998) 5-15.

[14] Q Guo, RE White. A steady-state impedance model for a PEMFC cathode, J. Electrochem. Soc. 151 (2004) E133-E149.

[15] AM Svensson, S Sunde, K Nisanhcioglu. Mathematical modeling of oxygen exchange and transport in air-perovskite-YSZ interface regions I. Reduction of intermediately adsorbed oxygen, J. Electrochem. Soc. 144 (1997) 2719-2732.

[16] AM Svensson, S Sunde, K Nisanhcioglu. Mathematical modeling of oxygen exchange and transport in air-perovskite-YSZ interface regions II. Direct exchange of oxygen vacancies, J. Electrochem. Soc. 145 (1998) 1390-1400.

[17] P Costamagna, P Costa, V Antonucci. Micro-modeling of solid oxide fuel cell electrodes, Electrochim. Acta. 43 (1998) 375-394.

[18] CW Tanner, KZ Fung, AV Virkar. Oxide fuel cell performance I. Theoretical analysis, J. Electrochem. Soc. 144 (1997) 21-30.

[19] SH Chan, XJ Chen, KA Khor. Cathode micromodel of solid oxide fuel cell, J. Electrochem. Soc. 151 (2004) A164-A172. 
[20] S Sunde. Simulations of Composite Electrodes in Fuel Cells, J. Electroceram. 5 (2000) 153-182.

[21] JR Macdonald, WB Johnson, Fundamentals of Impedance Spectroscopy, in: Macdonald JR (Ed.), Impedance Spectroscopy, first ed., Wiley, New York, NY, 1987, pp. $1-346$.

[22] Y Matsuzaki, I Yasuda. Electrochemical oxidation of $\mathrm{H} 2$ and $\mathrm{CO}$ in a $\mathrm{H} 2-\mathrm{H} 2 \mathrm{O}-\mathrm{CO}-$ $\mathrm{CO} 2$ system at the interface of a Ni-YSZ cermet electrode and YSZ electrolyte, J. Electrochem. Soc. 147 (2000) 1630-1635.

[23] GO Lauvstad, R Tunold, S Sunde. Electrochemical oxidation of CO on Pt and Ni point electrodes in contact with an yttria-stabilized zirconia electrolyte - I. Modeling of steady-state and impedance behavior, J.Electrochem.Soc. 149 (2002) E497-E505.

[24] AM Sukeshini, B Habibzadeh, BP Becker, CA Stoltz, BW Eichhorn, GS Jackson. Electrochemical oxidation of $\mathrm{H}_{2}, \mathrm{CO}$, and $\mathrm{CO} / \mathrm{H}_{2}$ mixtures on patterned $\mathrm{Ni}$ anodes on YSZ electrolytes, J.Electrochem.Soc. 153 (2006) A705-A715.

[25] JO Bockris, Reddy, A. K. N., M Gamboa-Aldeco, Modern Electrochemistry, Volume 2A, Fundamentals of Electrodics, second ed., Kluwer Academic/Plenum Publishers, New York, 2000.

[26] M Doyle, JP Meyers, J Newman. Computer simulations of the impedance response of lithium rechargeable batteries, J. Electrochem. Soc. 147 (2000) 99-110.

[27] TF Fuller, Solid-Polymer-Electrolyte Fuel Cells, (1992) 176.

[28] RV Homsy, J Newman. An asymptotic solution for the Warburg impedance of a rotating disk electrode, J. Electrochem. Soc. 121 (1974) 521-523.

[29] S Gewies, WG Bessler. Physically based impedance modeling of Ni/YSZ cermet anodes, J. Electrochem. Soc. 155 (2008) B937-B952.

[30] JS Newman, Electrochemical Systems, 2nd ed., Prentice-Hall, Englewood Cliffs, NJ, 1991.

[31] M Mogensen, T Lindegaard, UR Hansen, G Mogensen. Physical-Properties of Mixed Conductor Solid Oxide Fuel-Cell Anodes of Doped CeO2, J.Electrochem.Soc. 141 (1994) 2122-2128.

[32] E Ruiz-Trejo, J Maier. Electronic transport in single crystals of Gd-doped ceria, J. Electrochem. Soc. 154 (2007) B583-B587.

[33] CR Barrett, WD Nix, AS Tetelman, The Principles of Engineering Materials, first ed., Prentice Hall, Englewood Cliffs, NJ, 1973. 
[34] S Dikmen, P Shuk, M Greenblatt, H Gocmez. Hydrothermal synthesis and properties of $\mathrm{Ce}_{1-\mathrm{x}} \mathrm{Gd}_{\mathrm{x}} \mathrm{O}_{2 \text {-delta }}$ solid solutions, Solid State Sciences. 4 (2002) 585-590.

[35] BA Boukamp, M Verbraeken, DHA Blanck, P Holtappels. SOFC-anodes, proof for a finite-length type Gerischer impedance? Solid State Ionics. 177 (2006) 2539-2541.

[36] S Primdahl, M Mogensen, Gas conversion impedance: SOFC anodes in $\mathrm{H}_{2}-\mathrm{H}_{2} \mathrm{O}$ atmospheres, in: Stimming U, Singhal SC, Tagawa H, Lahnert W (Eds.), Proceedings of the Fifth International Symposium on Solid Oxide Fuel Cells, SOFC V, PV97-40, Pennington, NJ ed., The Electrochemical Society, Pennington, NJ, 1997, pp. 530-537.

[37] S Primdahl, M Mogensen. Gas diffusion impedance in characterization of solid oxide fuel cell anodes, J.Electrochem.Soc. 146 (1999) 2827-2833.

[38] S Primdahl, M Mogensen. Gas conversion impedance: A test geometry effect in characterization of solid oxide fuel cell anodes, J.Electrochem.Soc. 145 (1998) 24312438.

[39] W Weppner, Electrode performance, in: Bruce PG (Ed.), Solid State Electrochemistry, first ed., Cambridge University Press, Cambridge, UK, 1995, pp. 204.

[40] FA Kröger, HJ Vink, Relations between the concentrations of imperfections in crystalline solids, in: Seitz F, Turnull D (Eds.), Solid State Physics - Advances in Research and Applications, Academic Press, New York, NY, 1956, pp. 431-435.

[41] H Gerischer, Z. Phys. Chem. 198 (1951) 216.

[42] BA Boukamp, Bouwmeester, H. J. M. Interpretation of the Gerischer impedance in solid state ionics, Solid State Ionics. 157 (2003) 29-33.

[43] W Lai, SM Haile. Impedance spectroscopy as a tool for chemical and electrochemical analysis of mixed conductors: A case study of ceria, J. Am. Ceram. Soc. 88 (2005) 2979-2997.

[44] SB Adler. Factors governing oxygen reduction in solid oxide fuel cell cathodes, Chem. Rev. 104 (2004) 4791-4843.

[45] C Gabrielli, Identification of electrochemical processes by frequency response analysis, Technical Report \#004/83, Solartron Analytical, Farnborough, UK, 1984, pp. 36.

[46] SB Adler, XY Chen, JR Wilson. Mechanisms and rate laws for oxygen exchange on mixed-conducting oxide surfaces, J. Catalysis. 245 (2007) 91-109.

[47] JR Wilson, DT Schwartz, SB Adler. Nonlinear electrochemical impedance spectroscopy for solid oxide fuel cell cathode materials, Electrochim. Acta. 51 (2006) 1389-1402. 


\section{CHAPTER 5}

\section{ELECTROCHEMICAL IMPEDANCE SPECTROSCOPY

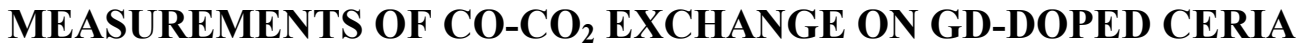 ELECTRODES}

\subsection{Introduction}

In this chapter, the experimental results of impedance measurements on GDC electrodes are presented. In addition, the viability of modeling of $\mathrm{CO}-\mathrm{CO}_{2}$ exchange on GDC will be assessed by fitting this experimental data to the $\mathrm{CO}-\mathrm{CO}_{2}$ exchange model, developed in Chapter 4, which considers exchange of oxygen between gas and solid, and bulk transport of oxygen ions to the electrolyte. This approach seems appropriate to this study as the bulk ionic conductivity of GDC is one of the highest known, as discussed in Chapter 3. A similar approach has worked for oxygen exchange on mixed-conducting SOFC cathode materials with the highest oxygen vacancy transport rates [1].

\subsubsection{Electrochemical Impedance Spectroscopy (EIS) measurements}

Electrochemical impedance spectroscopy (EIS) has become a common experimental technique to evaluate a wide range of electrochemical systems $[2,3]$ and in particular, solid state electrochemical systems [4]. In the solid oxide fuel cell area, EIS has been utilized extensively over the past 20 years [5], and most of recent works on $\mathrm{CO}$ oxidation [6-10] and $\mathrm{CO}_{2}$ reduction [11] do include impedance data on $\mathrm{Ni}-\mathrm{YSZ}$ and other electrodes for comparison with the experimental results presented here. 
Chapter 3 provided the details on the choice of $40 \mathrm{~mol} \%$ GDC as the electrode material and fabrication of the symmetric GDC electrode cells, along with a description of the experimental setup for testing the cells.

\subsection{Analysis and data reduction}

\subsubsection{The $\mathrm{CO}-\mathrm{CO}_{2}$ exchange model}

The CO- $\mathrm{CO}_{2}$ exchange electrode model used to fit the experimental impedance data consists of a one-dimensional mixed conducting porous electrode in which bulk diffusion and surface exchange are the two chemical process phenomena occurring in the electrode. As discussed in detail in Chapter 4, the $\mathrm{CO}-\mathrm{CO}_{2}$ exchange model derived results in the following relationship for the impedance, $Z_{\text {chem }}$, due to these chemical processes:

$Z_{\text {chem }}=\frac{R_{\text {chem }}}{\sqrt{1+j \omega t_{\text {chem }}} \tanh \left(\phi \sqrt{1+j \omega t_{\text {chem }}}\right)}$

where $R_{\text {chem }}$ is a characteristic resistance, $t_{\text {chem }}$ is a characteristic time constant, $j=\sqrt{-1}$, $\omega$ is the radial (or angular) frequency, and $\phi=\frac{L}{l_{\delta}}$ where $l_{\delta}$ is a characteristic length related to the size of the utilization (or active) region of the electrode in the limit of 
infinite $L . R_{\text {chem }}, t_{c h e m}$, and $l_{\delta}$ are related to the material properties of the mixed conducting electrode as follows:

$R_{\text {chem }}=\frac{R T}{2 F^{2}} \sqrt{\frac{1}{(1-\varepsilon) c_{v} D_{v}^{\text {eff }} a \Re_{0}}}$

$t_{\text {chem }}=\frac{c_{v}(1-\varepsilon)}{A a \Re_{0}}$

$l_{\delta}=\sqrt{\frac{(1-\varepsilon) x_{v}^{e q b} C_{0} D_{v}^{e f f}}{a \Re_{0}}}$

where $x_{v}^{\text {eqb }}$ is the vacancy concentration at equilibrium temperature and $P_{O_{2}}$ conditions, $c_{0}$ is the concentration of oxygen lattice sites, $D_{v}^{\text {eff }}\left(=\frac{D_{v}}{\tau_{s}}\right)$ is the effective vacancy diffusion coefficient, $\varepsilon$ is the porosity, $a$ is the surface area per unit volume, $\tau_{s}$ is the solid-phase tortuosity, and $\Re_{0}$ is the equilibrium surface exchange rate for the $\mathrm{CO}-\mathrm{CO}_{2}$ exchange reaction. The thermodynamic factor $A[12]$ is defined as:

$$
A=-\frac{1}{2} \frac{\partial \ln P_{\mathrm{O}_{2}}}{\partial \ln x_{v}^{e q b}}
$$

This factor can be derived from a defect model based on oxygen non-stoichiometric measurements; Appendix D provides a derivation of an ideal defect model and the resulting expression for $A$ used in this work. 
The impedance data was fitted to Equation (5-1) with a non-linear least squares fit using the Levenberg-Marquardt algorithm [13] implemented in the commercial mathematical software package, MATLAB ${ }^{\circledR}$. The objective function used to fit the complex variable data for least squares minimization is [14]:

$$
F_{\min }=\sum_{i=1}^{n}\left|\operatorname{Re}\left(Z_{i}^{\text {data }}-Z_{i}^{\text {fit }}\right)+\operatorname{Im}\left(Z_{i}^{\text {data }}-Z_{i}^{\text {fit }}\right)\right|^{2}
$$

where $F_{\min }=$ objective function for minimization

$Z_{i}^{\text {data }}=$ individual complex data value for an experimental impedance spectra data

$Z_{i}^{\text {fit }}=$ fitted impedance value corresponding to an experimental data point $Z^{\text {fit }}=Z^{\text {fit }}(\omega, \lambda)=$ complex-valued impedance function to be fitted, i.e. Equation (5-1)

$\lambda=$ vector of fitting parameters, i.e. $\left[R_{\text {chem }}, t_{\text {chem }}, \phi\right]$ for Equation (5-1)

$\omega=$ dimensionless frequency (single independent variable)

$n=$ total number of experimental data points for an individual impedance spectrum

The resulting fits of the impedance data provide a set of values for the $R_{\text {chem }}, t_{\text {chem }}$, and $\phi$ parameters for each individual spectrum. These values were utilized in Equations (5-2), (5-3), and (5-4), to extract values for $D_{v}$ and $\mathfrak{R}_{0}$ for direct comparison with similar material property data in the literature. An example MATLAB script (or m-file) for performing the fit and other reducing data functions can be found in Appendix I. 


\subsection{Results and Discussion}

\subsubsection{Electrode microstructure parameters}

Figure 5-1 is an SEM of the typical GDC electrode microstructure. Sufficient adhesion on GDC to YSZ was obtained and no de-lamination of the GDC layer was observed over 3 weeks of operation in low $\mathrm{O}_{2}$ partial pressure atmospheres with 12-15 thermal cycles over a temperature range of $700^{\circ}-950^{\circ} \mathrm{C}$. A thin layer can be seen at the GDC/YSZ interface, which could possibly be an interdiffusion layer, consistent with findings of other workers, who found that $\mathrm{Ce}^{4+}$ inter-diffuses into YSZ at sintering temperatures above $1100^{\circ} \mathrm{C}$ [15]. The GDC layer is a porous structure of sintered GDC particles, typically $0.5-1 \mu \mathrm{m}$ with the largest $3-5 \mu \mathrm{m}$ in size. The thickness of the electrode is $40-50 \mu \mathrm{m}$.

For application of the $\mathrm{CO}-\mathrm{CO}_{2}$ exchange model, three properties need to be estimated from the electrode microstructure: porosity, surface area per unit volume, and tortuosity. The porosity is estimated to be 0.50 using software employing the point-count method [16]. The porosity is not particularly uniform, especially when compared to state-of-the-art fabrication achievable in most solid state electrochemistry labs where screen-printing, sputtering, or tape casting are the typical electrode layer application methods. The surface area per unit volume, $a$, is estimated at $10,000 \mathrm{~cm}^{-1}$ by comparison with SEM images of a sectioned Ni-YSZ electrode with similar electrode microstructure [17]. 


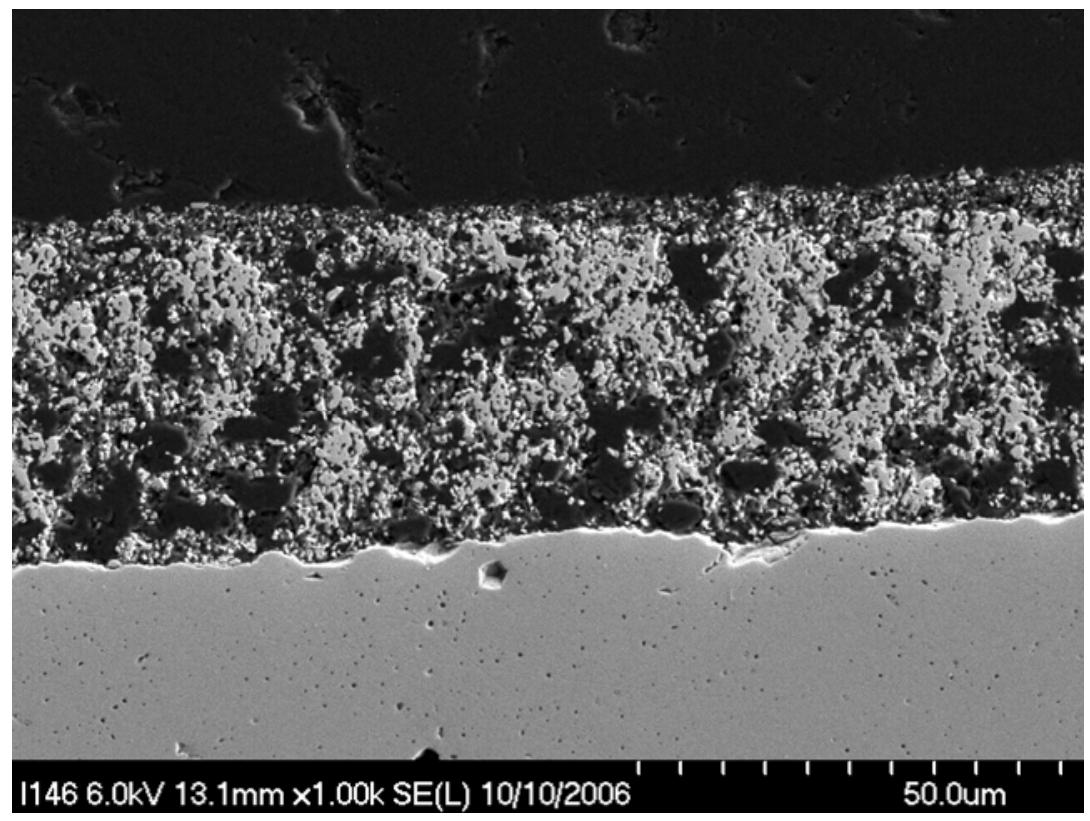

(a)

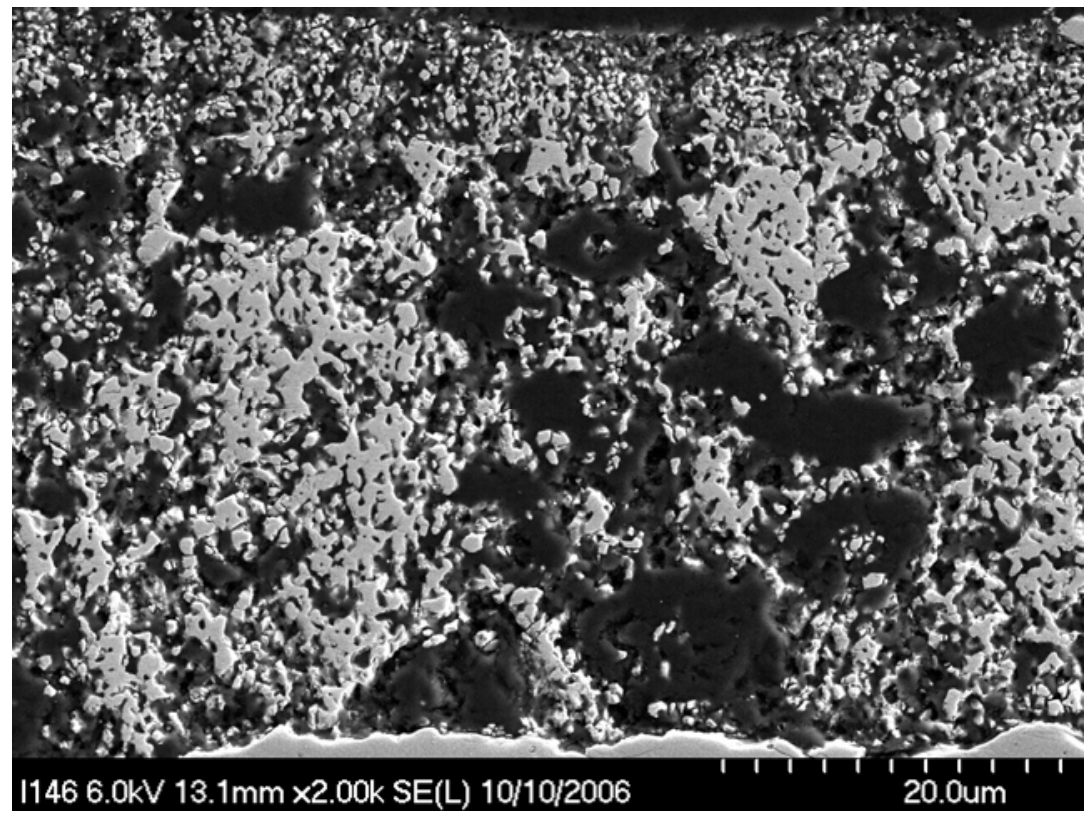

(b)

Figure 5-1. Scanning electron micrographs of porous $\mathrm{Gd}_{0.4} \mathrm{Ce}_{0.6} \mathrm{O}_{1.75}$ electrode fired onto a YSZ electrolyte pellet at $1250^{\circ} \mathrm{C}$. Sample was impregnated with an epoxy before being cut and polished. a) image of porous electrode layer and YSZ electrolyte; b) close-up of GDC electrode layer. 
This value is on the lower end of the $10,000-30,000 \mathrm{~cm}^{-1}$ typically achieved in conventional Ni-YSZ composite anodes and LSM cathodes. The low $a$ value is mainly due to high temperature and long sintering times to circumvent problems with adherence of our porous GDC layer with the YSZ electrolyte and Pt current collector. Other researchers have avoided these problems by using alternative fabrication approaches, specifically aqueous impregnation of ceria into a porous YSZ layer [18]. The solid-phase tortuosity of the electrode, $\tau_{s}$, is estimated to be 1.5 based on values estimated for SOFC electrodes in the literature [19].

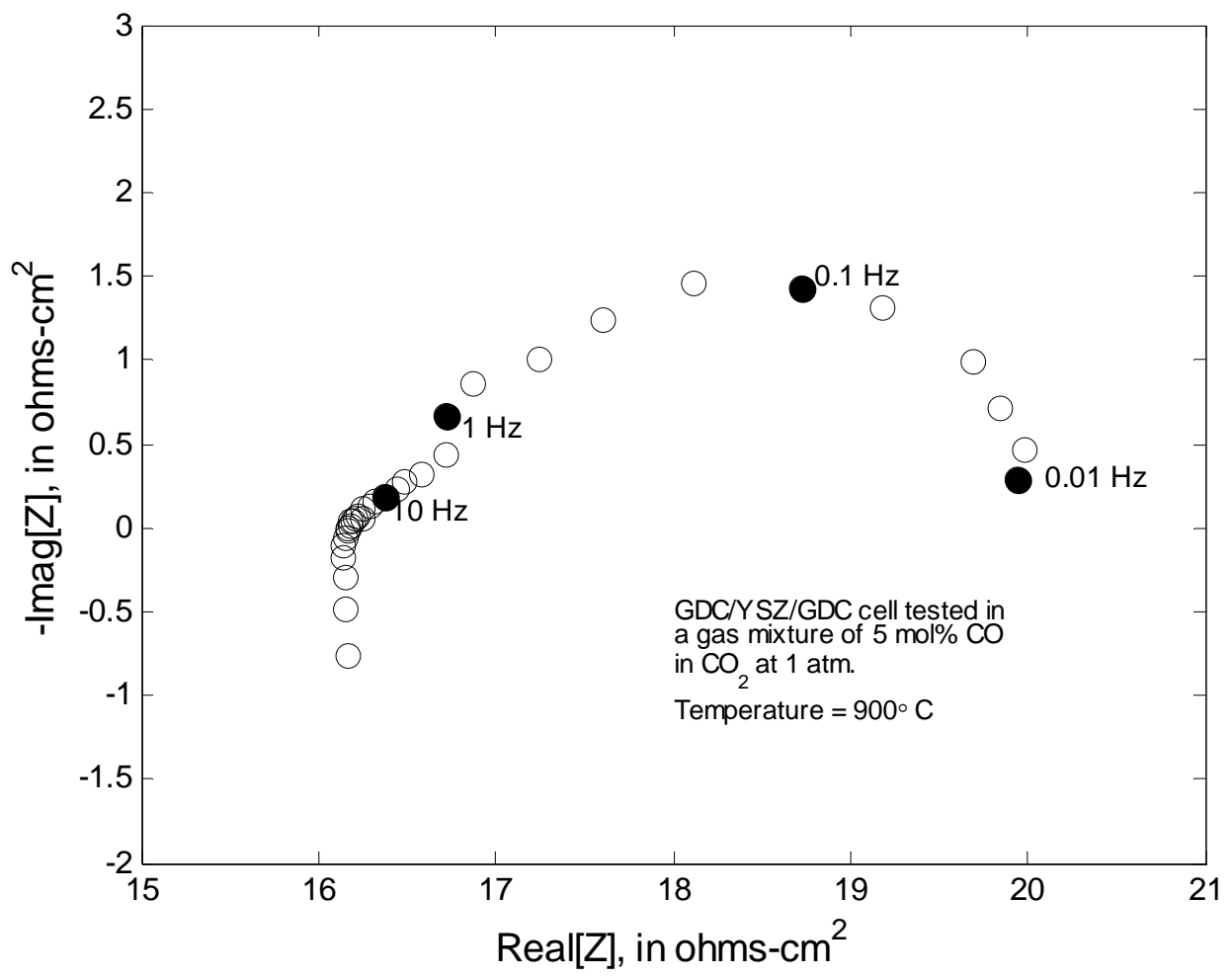

Figure 5-2. Nyquist plot of AC impedance spectrum of GDC/YSZ/GDC cell after 103 hours of operation. 


\subsubsection{Impedance measurement results}

Figure 4 displays a typical impedance response of a GDC/YSZ/GDC cell at $900^{\circ} \mathrm{C}$ in a $5 \mathrm{~mol} \% \mathrm{CO}$ in $\mathrm{CO}_{2}$ gas mixture. Above frequencies of $600 \mathrm{~Hz}$, a purely inductive response (i.e. positive imaginary impedance values) is observed and attributed primarily to ohmic losses from the electrolyte and test leads. Below this high frequency threshold, the mid-range frequencies display an arc at $\sim 45^{\circ}$ to the real and imaginary axes. At low frequencies $(<1 \mathrm{~Hz})$, a large depressed semicircle is the dominant feature. The transition from the mid-range arc to the low frequency arc is sharp, indicating separate phenomena are responsible; spectra with a similar sharp transition, along with a large low frequency semi-circle response, has also been observed on $10 \mathrm{~mol} \%$ GDC electrodes in $\mathrm{H}_{2}-\mathrm{H}_{2} \mathrm{O}$ [20]. Also, some scatter is evident in the $1 \mathrm{~Hz}$ frequency range, consistent with observations of CO oxidation work on Ni-YSZ electrodes [7,10,21]. This response was recorded after 103 hours of operation; the area specific resistance (ASR) decreased and stabilized after the first 100 hours of operation. The low frequency arc did not intersect the real axis, although spectra were measured down to $0.01 \mathrm{~Hz}$; this is similar to Holtappels et al. [7] on Ni-YSZ and Mizusaki et al. [22] on Pt, but contrasts with the low frequency inductance observed by Lauvstad et al. [9] for Ni point electrodes on YSZ.

\subsubsection{Electrode activation energy}

Figure 5-3 displays an Arrhenius plot of area-specific-resistance (ASR) over the entire test matrix of impedance measurements. The ASR was calculated by subtracting the high 


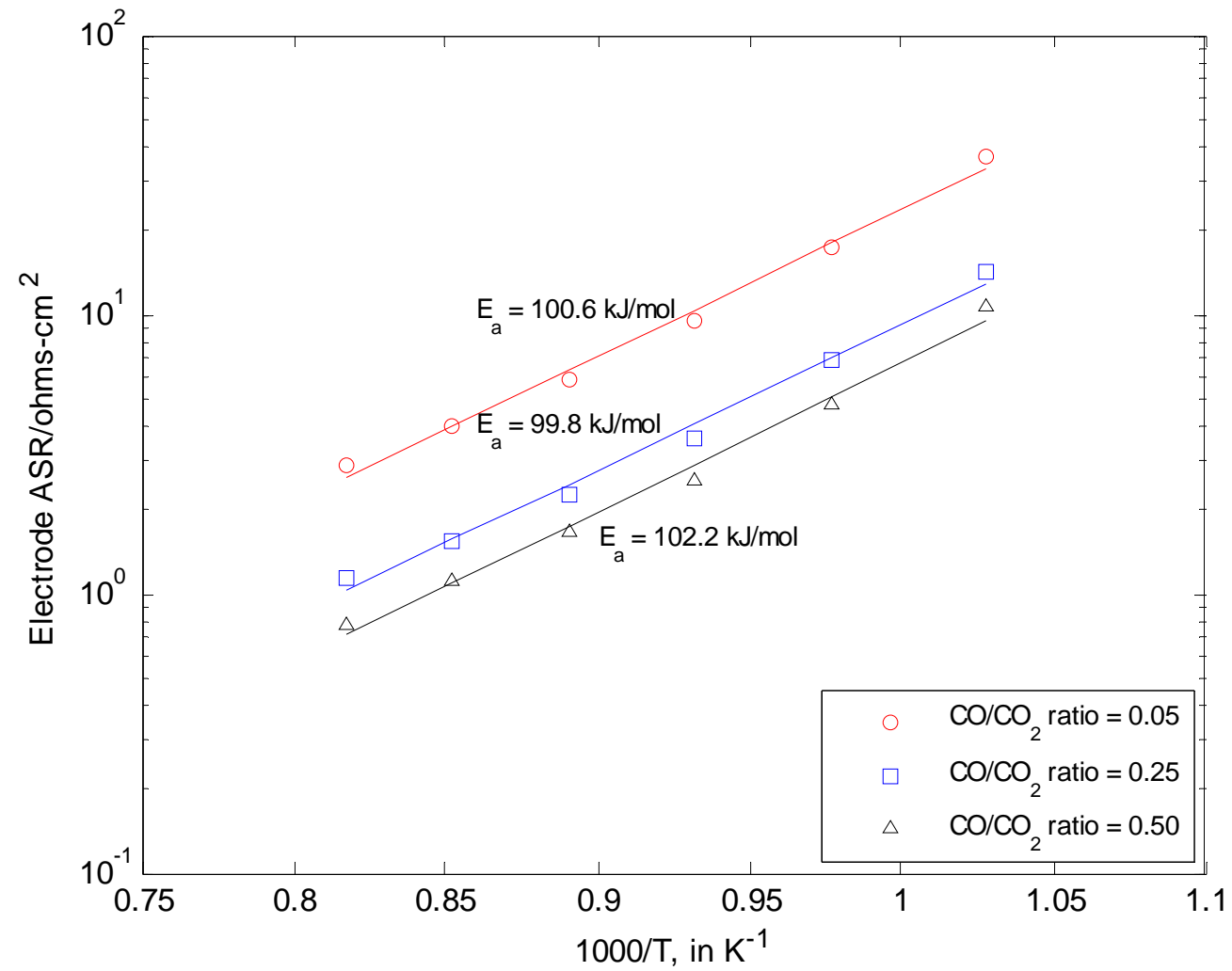

Figure 5-3. Arrhenius plot of area specific resistance (ASR) over the range of temperature and $\mathrm{CO} / \mathrm{CO}_{2}$ gas mixture ratios tested.

frequency intercept (i.e. the ohmic resistance component) from the low frequency intercept of the real axis, i.e. $\operatorname{Re}\left[Z-R_{\text {ohmic }}\right]$. Since the low frequency data did not intersect the real axis, the data were extrapolated with the depressed semi-circle fit in ZView $^{(}$instrumentation software (Scribner Associates). The Arrhenius plot shows good linearity over the wide range of temperature, with the linear regression fit giving an activation energy of $\sim 100 \mathrm{~kJ} / \mathrm{mol}$. No significant variation in activation energy with $\mathrm{CO} / \mathrm{CO}_{2}$ gas mixture ratio was observed. No activation energy data for carbon dioxide reduction on ceria or Ni-YSZ was found in the literature, but the activation energy for our 
experimental data does lie between the $172 \mathrm{~kJ} / \mathrm{mol}$ for a fixed $P_{\mathrm{O}_{2}}=10^{-15}$ atm and 55.0 $\mathrm{kJ} / \mathrm{mol}$ in a fixed $\mathrm{CO} / \mathrm{CO}_{2}$ gas mixture ratio of 0.001 reported by Sprague et al. [23] on porous $\mathrm{Pt} / \mathrm{GdCaTi}_{2} \mathrm{O}_{7}$ cells.

\subsection{4 $\mathrm{CO}-\mathrm{CO}_{2}$ exchange model fitted parameters}

Figure 5-4 (a) displays the variation in impedance response for three different $\mathrm{CO} / \mathrm{CO}_{2}$ gas mixture ratios. The low frequency semicircular arc increases in size with lower $\mathrm{CO} / \mathrm{CO}_{2}$ ratio; which is consistent with observations by Mizusaki et al. [22] on $\mathrm{Pt} / \mathrm{YSZ} / \mathrm{Pt}$ cells, and Lauvstad et al. [6] on $\mathrm{SrFeO}_{3} / \mathrm{YSZ}$ cells, in a similar range of $\mathrm{CO} / \mathrm{CO}_{2}$ gas mixtures. There is evidence that the $\mathrm{CO}$ oxidation reaction rate on ceria $[24,25]$ and other oxide catalysts decreases when the reactant gas contains $\mathrm{CO}_{2}$. This may be due to preferential adsorption, or a higher activation energy of desorption of $\mathrm{CO}_{2}$, on the active catalyst sites of oxide and noble metal catalysts, and, the frequency range of this impedance arc would be consistent with a gas adsorption/desorption limiting behavior. [As an aside, Shao and Haile [26] have reported reduced oxygen reduction reaction rates on a promising oxide cathode material, $\mathrm{Ba}_{0.5} \mathrm{Sr}_{0.5} \mathrm{Co}_{0.8} \mathrm{Fe}_{0.2} \mathrm{O}_{3-\delta}$ (BSCF), in $\mathrm{O}_{2}$ gas mixtures with $\mathrm{CO}_{2}$ concentrations as low as $5 \%$.]

Figure 5-4 (a) also displays the fit of each individual spectra to Equation (5-1). Figure 5-4 (b) displays the real and imaginary parts of the impedance data and the corresponding fit as a function of frequency for one of the spectra in (a); the purpose 


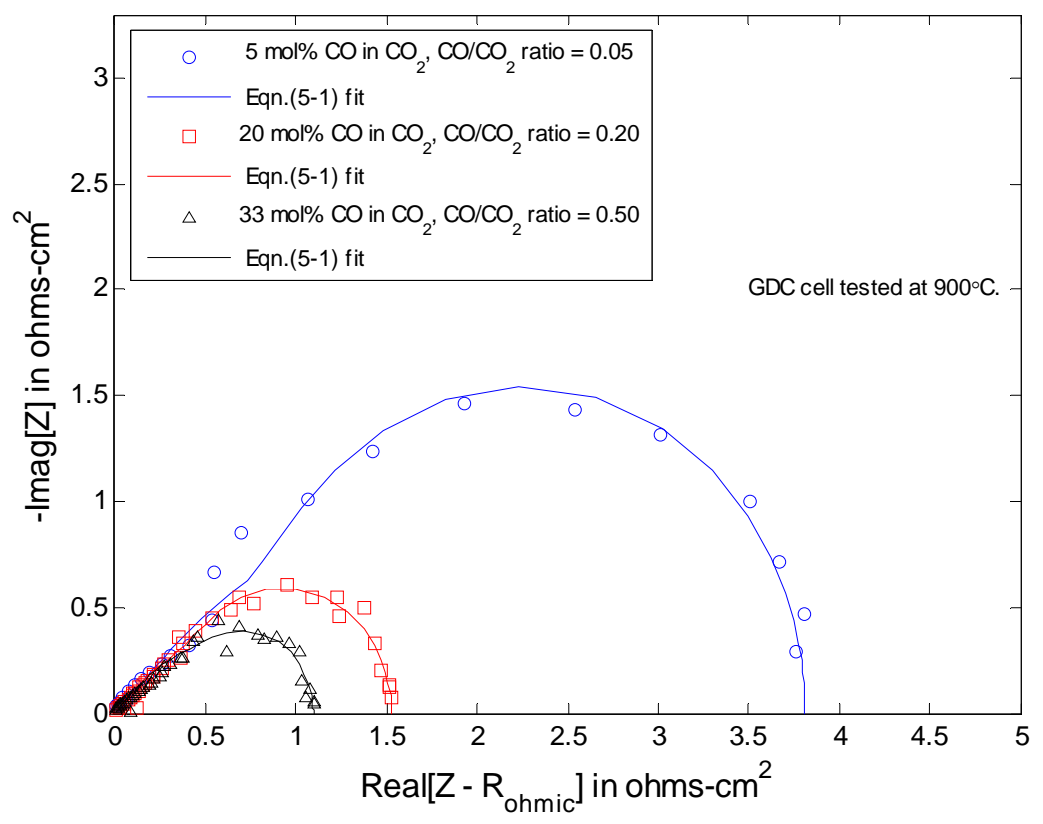

(a)

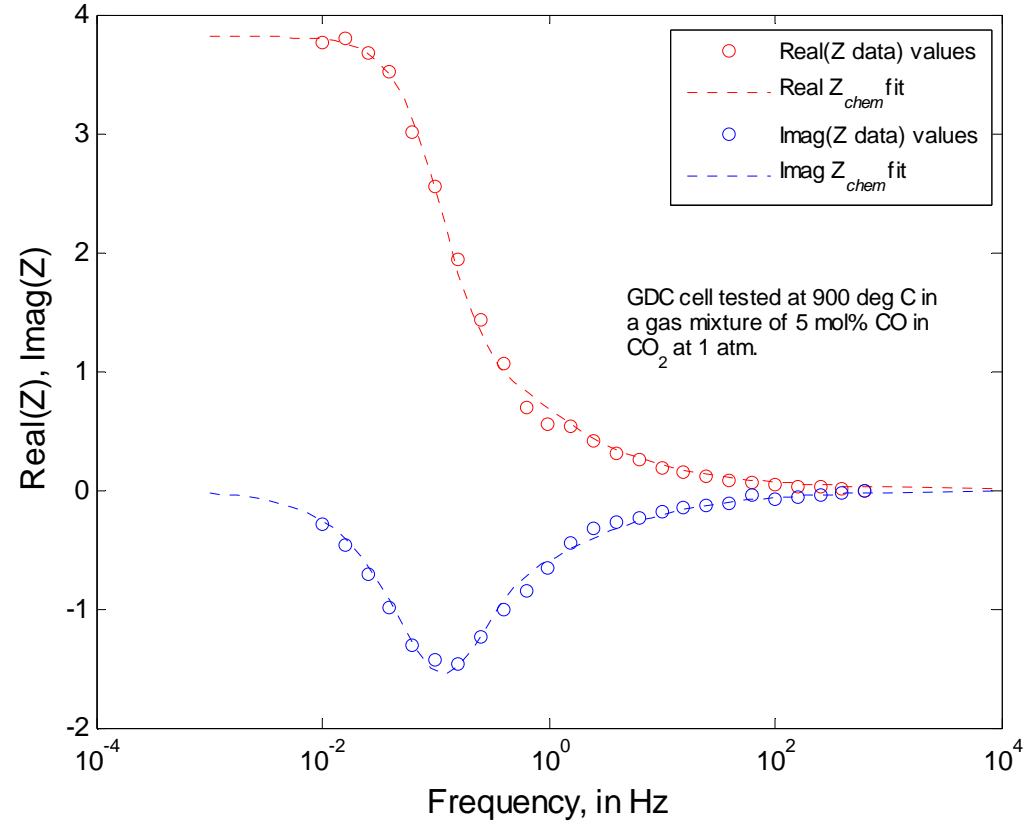

(b)

Figure 5-4. (a) AC impedance spectra responses with variation in $\mathrm{CO} / \mathrm{CO}_{2}$ gas mixture ratio. High frequency impedance response, $R_{\text {ohmic }}$, has been subtracted from the real impedance values on the abscissa. (b) Fit of Equation (5-1) to the real and imaginary parts of $\mathrm{Z}$ for a set of impedance spectra data. 
being to show how well the fit is for the minimization of the objective function defined in Equation (5-6). The fit is generally very good with the $\operatorname{Im}[Z]$ data showing the largest deviation. The norm of the residuals was less than 0.04 for these three sets of data.

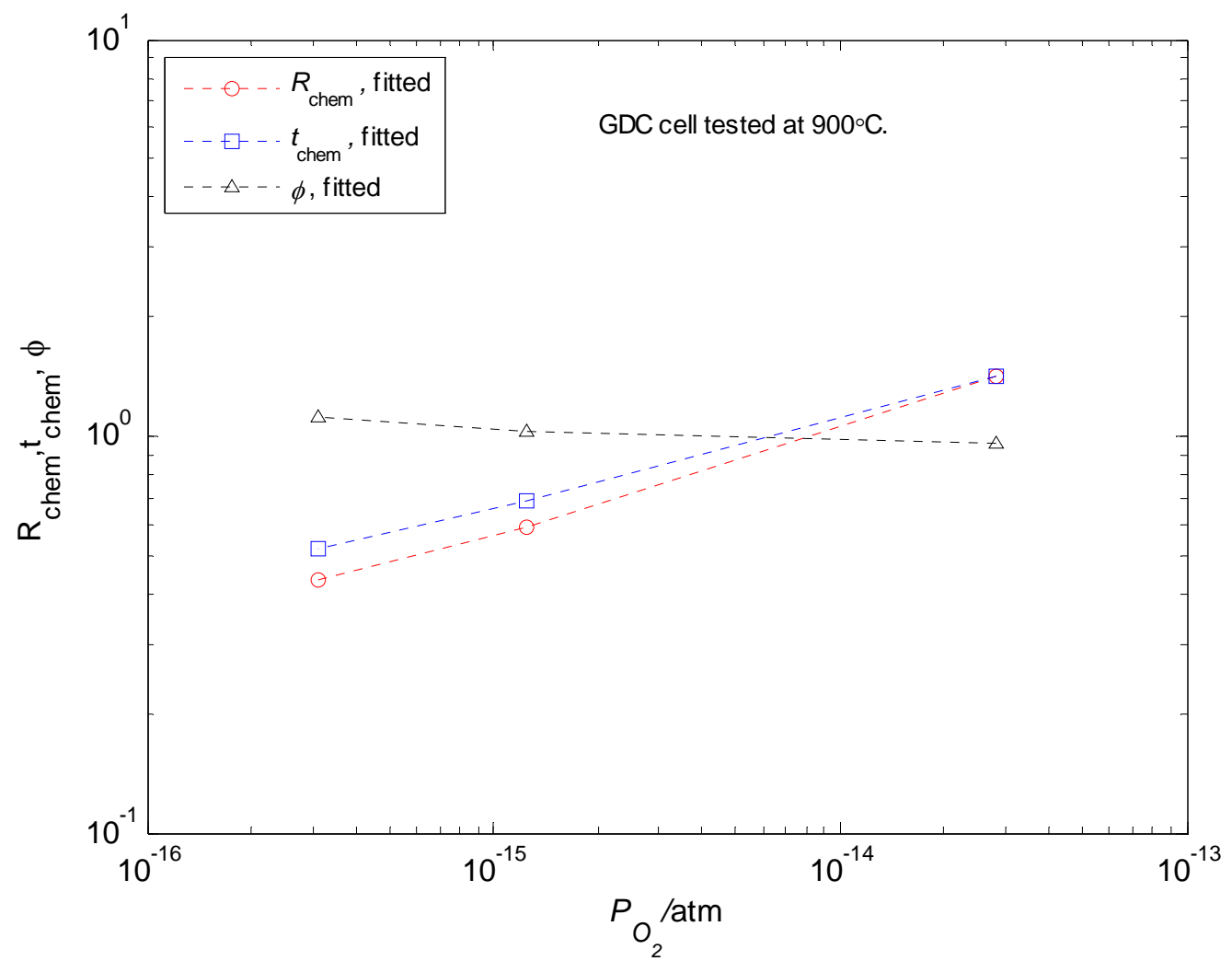

Figure 5-5. Fitted parameters from $\mathrm{CO}-\mathrm{CO}_{2}$ exchange model, Equation (5-1), as a function of partial pressure of $\mathrm{O}_{2}$. Data were extracted from GDC electrode impedance spectra for the $3 \mathrm{CO} / \mathrm{CO}_{2}$ ratios tested.

The fitted parameters for equation (5-1) are shown in Figure 5-5 as a function of $P_{\mathrm{O}_{2}}$ and in Figure 5-6 as a function of reciprocal temperature. The $R_{\text {chem }}$ and $t_{\text {chem }}$ trend as expected, with $R_{\text {chem }}$ being somewhat analogous to ASR, and $t_{\text {chem }}$ being a measure of the 
kinetic activity of the electrode. The utilization thickness ratio, $\phi$, a relative measure of the thickness of the electrode layer that is kinetically active is on the order of 1.0, i.e. $l_{\delta} \approx L$. As described in Chapter 4, the model indicates that for $\phi$ values in this range, the electrode is transitioning from co-limited (i.e. kinetic and diffusion limited) to kinetically limited behavior. Because of the insensitivity of the Tanh term to $\phi$ as $\phi \rightarrow 0$ in Equation (5-1), the model fit procedure was adjusted to fit $R_{\text {chem }}$ and $t_{c h e m}$, calculate values for $\phi$ from equation (5-4), and iterate on $\phi$ until the value for $\phi$ no longer changed. As Figure 5-5 indicates, $\phi$ does increase slightly with decreasing $P_{\mathrm{O}_{2}}$, possibly due to improving exchange kinetics for increasing $\mathrm{CO} / \mathrm{CO}_{2}$ ratios.

Figure 5-6 displays our fitted parameters with reciprocal temperature for a constant $\mathrm{CO} / \mathrm{CO}_{2}$ ratio of 0.50 . Both $R_{\text {chem }}$ and $t_{\text {chem }}$ decrease with increasing temperature in a nearly Arrhenius manner. The $\phi$ parameter appears to be a weak function of temperature; a linear regression fit of our data over the temperature range tested gives a slope of nearly zero, corresponding to $E_{a}<10 \mathrm{~kJ} / \mathrm{mol}$. The average of the $\phi$ values is $\sim 1.4$, i.e. slightly greater than 1.0 , also indicating the electrode is transitioning to kinetic limited behavior. Similar to Figure 5-5, the $\phi$ values were calculated using Equation (5-4) using the iterative fitting procedure described in the previous paragraph. At the lower temperatures, the norm of the residuals is noticeably larger $\left(\sim 4.0\right.$ at $\left.T=700^{\circ} \mathrm{C}\right)$ due to increased scatter in the low frequency data, and, as previously noted may be due to instability in the $\mathrm{CO}-\mathrm{CO}_{2}$ exchange reaction at these temperatures. 


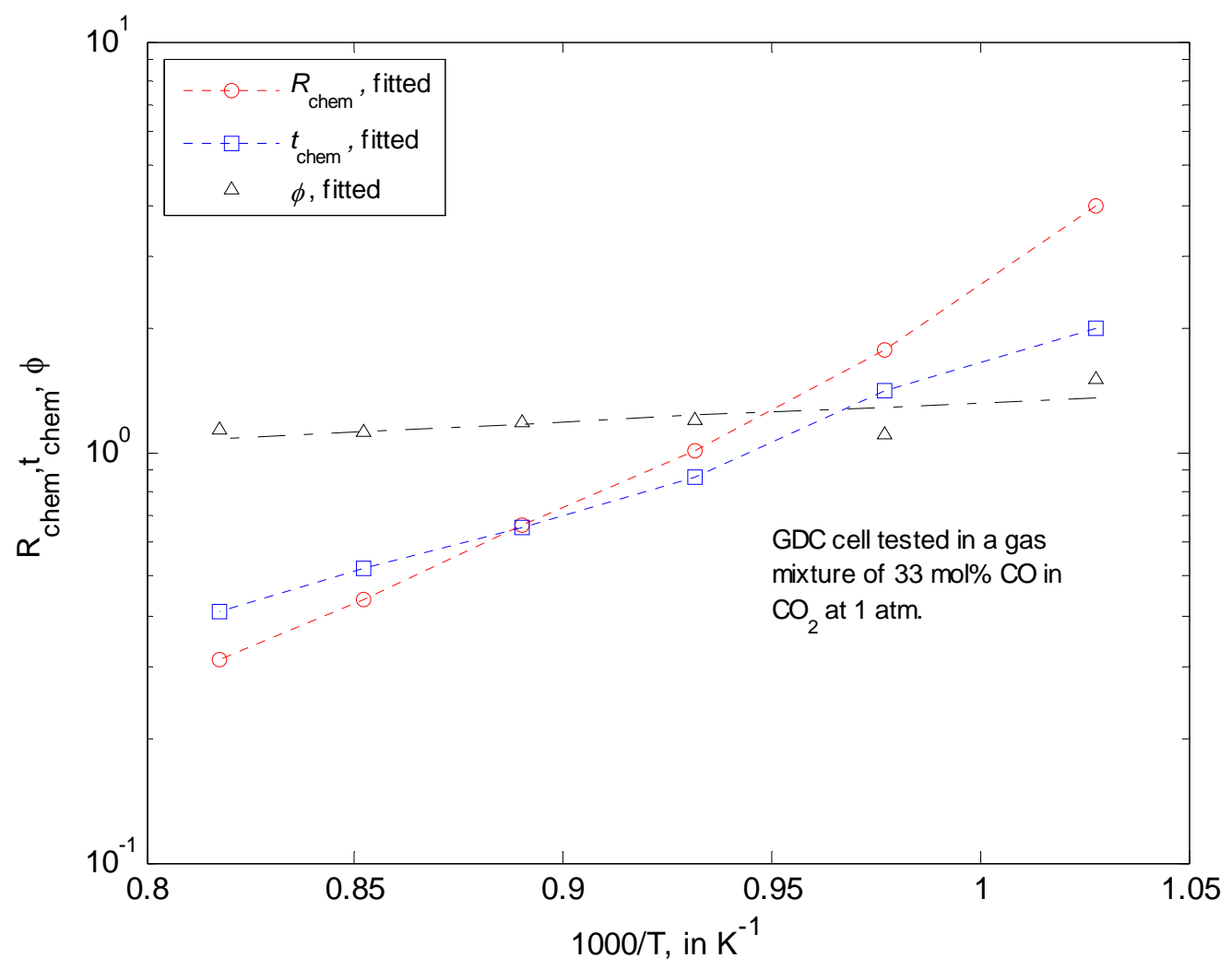

Figure 5-6. Fitted parameters for the $\mathrm{CO}-\mathrm{CO}_{2}$ exchange model, Equation (5-1), extracted from GDC electrode impedance spectra, as a function of reciprocal temperature.

\subsubsection{Electrode material properties extracted from the impedance data}

In order to compare these measurements with material properties measured in the literature using alternate techniques, two material properties, the vacancy diffusion coefficient $\left(D_{v}\right)$ and the exchange rate coefficient $\left(\Re_{0}\right)$ were extracted from Equations (5-2) and (5-3) using the fitted $R_{\text {chem }}$, and $t_{\text {chem }}$ data. Vacancy concentration values were calculated using an oxygen non-stoichiometric expression from an ideal point defect model derived in Appendix D: 
$\Delta G_{f i t}^{0}=-R T \ln \frac{4 \delta^{\prime 2}\left(\delta^{\prime}+\delta_{0}\right) P_{o_{2}}^{1 / 2}}{\left(1-2 \delta^{\prime}-2 \delta_{0}\right)^{2}\left(2-\delta^{\prime}-\delta_{0}\right)}$

where $\delta_{0}=$ deviation from stoichiometry due to Gd doping mole fraction

$\delta^{\prime}=$ deviation from stoichiometry due to $P_{\mathrm{O}_{2}}$

$\Delta G_{f i t}^{0}=$ fitted parameter $(\mathrm{J} / \mathrm{mol})$

Oxygen non-stoichiometry data for GDC were fitted to Equation (5-7) from linear sweep voltammetry measurements performed on 40 mol\% GDC in an oxygen pumping cell [27]. To calculate the thermodynamic factor, the following relationship was derived from Equation (5-7) for $A$ as defined in Equation (5-5):

$A=-\frac{1}{2} \frac{\partial \ln P_{O_{2}}}{\partial \ln x_{v}}=\frac{\delta_{0}}{\delta^{\prime}}-\frac{\delta_{0}+1}{2 \delta_{0}+2 \delta^{\prime}-1}-\frac{1}{\delta_{0}+\delta^{\prime}-2}$

where $x_{v}=\left(\delta_{0}+\delta^{\prime}\right) / 2$

\subsubsection{Vacancy diffusion coefficient}

Figure 5-7 displays the extracted $D_{v}$ data as a function of reciprocal temperature for our measurements in a gas mixture ratio of $\mathrm{CO} / \mathrm{CO}_{2}=0.50$. An activation energy of $115 \mathrm{~kJ} / \mathrm{mol}$ was determined from a linear regression fit, which is in close agreement with the $\sim 112 \mathrm{~kJ} / \mathrm{mol}$ reported by other workers [29] for the bulk ionic conductivity of 40 mol\% GDC in air. In order to compare more directly with the experimental data, $D_{v}$ 
values were estimated from the reference [29] conductivity data using the Nernst-Einstein equation [30]:

$D_{v}=\frac{R T \sigma_{i}}{4 c_{v} F^{2}}$

where $\sigma_{i}$ is the ionic conductivity of GDC in air.

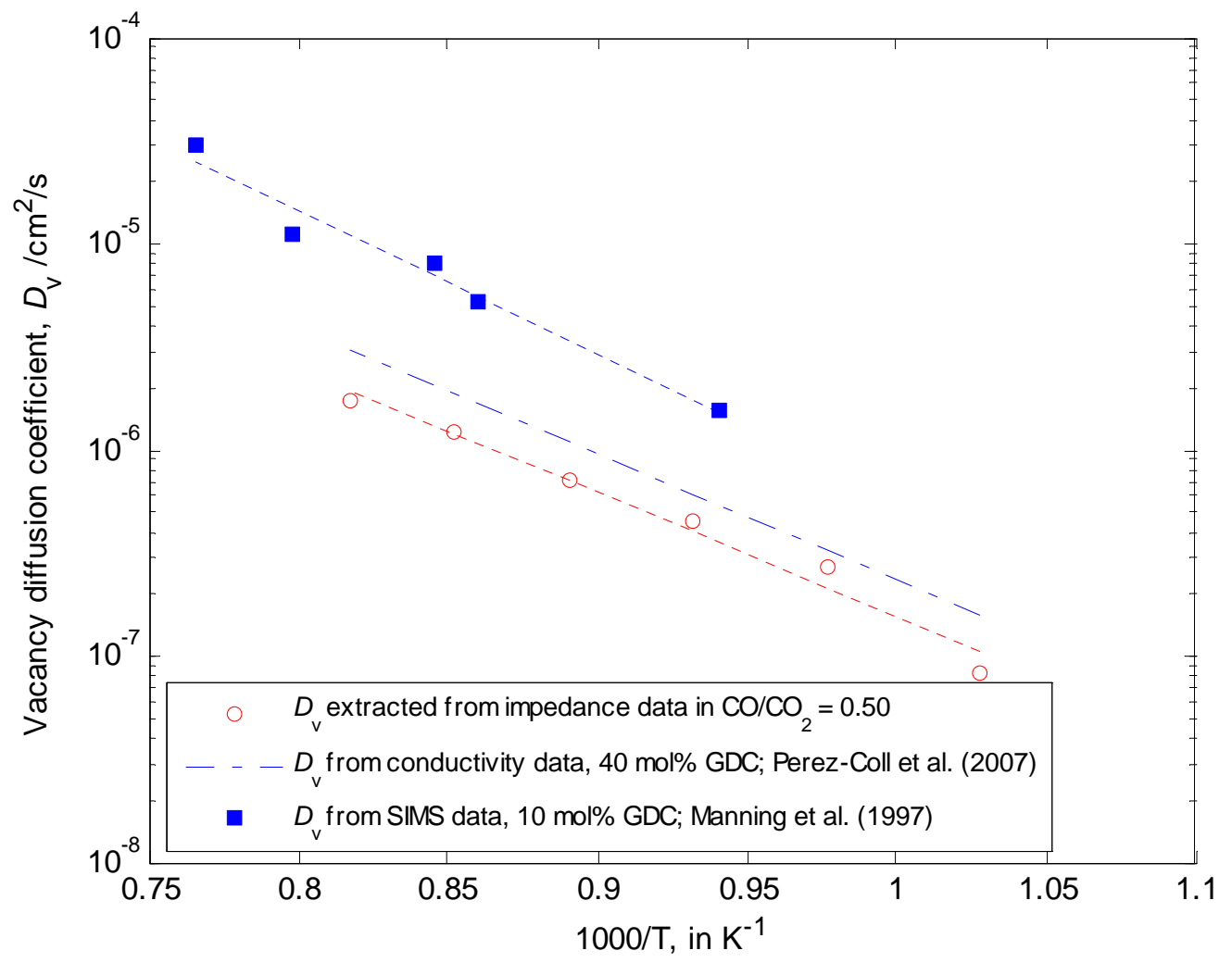

Figure 5-7. $D_{\mathrm{v}}$ data as a function of reciprocal temperature, extracted from measured impedance data on a $40 \mathrm{~mol} \%$ GDC electrode. The activation energy is $\sim 115 \mathrm{~kJ} / \mathrm{mol}$. Literature data are from IEDP-SIMS measurements on $10 \mathrm{~mol} \% \mathrm{GDC}$ in ref. [28] and conductivity measurements on $40 \mathrm{~mol} \% \mathrm{GDC}$ in air in ref. [29]. 
The extracted $D_{v}$ data is roughly $50 \%$ lower than what was estimated from the conductivity data. This difference could possibly be due to increased grain boundary resistance in our GDC electrodes. The reference [29] data were measured on GDC samples sintered at higher temperatures $\left(1600^{\circ} \mathrm{C}\right.$ vs. $\left.1250^{\circ} \mathrm{C}\right)$ and for longer times $(10$ hours vs. 2 hours); in addition, a sintering additive (Co) was used in processing their sample pellets. Another possible reason is the uncertainty in the estimate of $\tau_{s}$ for the GDC electrode microstructure used in this study.

As noted in Chapter 3, Dikman et al. [31] reports a maximum in ionic conductivity, and a corresponding minimum in the activation energy for GDC at $x=0.25$. In order to compare our $D_{v}$ data with data for lower doped GDC, self-diffusion coefficient $\left(D^{*}\right)$ data from Isotope Exchange/Depth Profiling (IEDP) using Secondary Ion Mass Spectroscopy (SIMS) measurements on $10 \mathrm{~mol} \%$ GDC in the literature [28] was converted to $D_{v}$ data with equation (5-10) [32]:

$D_{v}=\frac{2-\delta}{\delta f} D^{*}$

where $f$ is a correlation factor equal to $0.6531 .^{*}$

\footnotetext{
* Equation (5-10) assumes random walk theory [50] and $f$ represents the deviation from
} randomness of the atomic jumps; $f$ has a theoretical value of 0.6531 for a simple cubic lattice [51]. 
As can be seen in Figure 5-7, this data is about an order of magnitude greater than the $D_{v}$ values estimated from our impedance data, and the activation energy of $87 \mathrm{~kJ} / \mathrm{mol}$ on $10 \mathrm{~mol} \%$ GDC is less than our $114 \mathrm{~kJ} / \mathrm{mol}$ measured value on $40 \mathrm{~mol} \% \mathrm{GDC}$, consistent with the general observations on GDC conductivity noted above.

Figure 5-8 displays the extracted $D_{v}$ values vs. $P_{\mathrm{O}_{2}}$, and indicates a slight increase in $D_{v}$ with decreasing $P_{\mathrm{O}_{2}}$. But previous workers $[32,33]$ have reported no variation with $P_{\mathrm{O}_{2}}$ on GDC, at least at $10 \mathrm{~mol} \%$ or lower Gd doping levels. $D_{v}$, from a theoretical perspective, is inversely proportional to $c_{v}[34]$, opposite to the trend observed in Figure 5-8. The data from Yashiro et al. [32] is presented in Figure 5-8 and has a similar trend to ours, but they discount the relationship to $P_{\mathrm{O}_{2}}$, noting the uncertainty in their measurement of this property. The variation in the data (for example $D_{v}=0.2-0.5 \times$ $10^{-6}$ at $850^{\circ} \mathrm{C}$ ) is within their observed variation over a similar range of $P_{\mathrm{O}_{2}}$; and as such, no conclusions are made here on the variation in $D_{v}$ with $P_{\mathrm{O}_{2}}$ in $40 \mathrm{~mol} \%$ GDC. Kilner [34] briefly describes examples of non-ideal phenomena, such as defect clustering, that can occur in highly doped fluorite oxides like GDC, but none of these explanations qualitatively provide a rationale for the apparent trend. Figure 5-8 also includes a value for $D_{v}$ computed from ionic conductivity data at $800^{\circ} \mathrm{C}$ using Equation (5-9), which indicates reasonable agreement with the extracted $D_{v}$ data. 


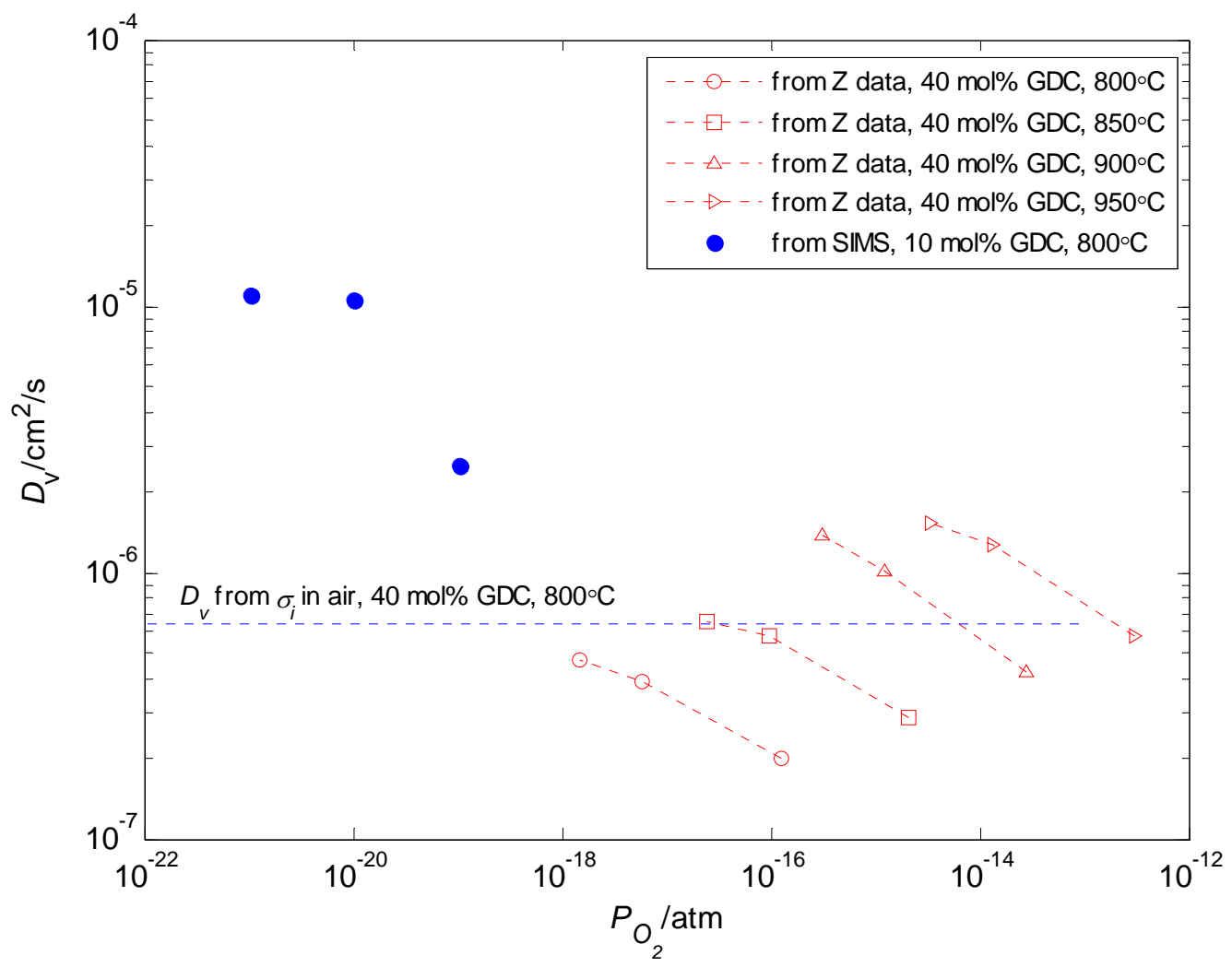

Figure 5-8. $D_{v}$ data as a function of the partial pressure of $\mathrm{O}_{2}$, extracted from measured impedance data on a $40 \mathrm{~mol} \%$ GDC electrode. Literature data (filled blue circles) is from IEDP-SIMS measurements on $10 \mathrm{~mol} \%$ GDC in ref. [32]. $D_{v}$ for $40 \mathrm{~mol} \%$ GDC is a single data point calculated using equation (5-9) from conductivity data in ref. [29].

\subsubsection{Surface exchange rate}

Figure 5-9 displays the extracted $\mathfrak{R}_{0}$ data obtained using Equation (5-2), as a

function of $P_{\mathrm{O}_{2}}$ for three temperatures. As a comparison, exchange rate data were derived from surface reaction rate constant $(k)$ data [32] for surface exchange measurements on $10 \mathrm{~mol} \% \mathrm{GDC}$, in both $\mathrm{CO}-\mathrm{CO}_{2}$ and $\mathrm{H}_{2}-\mathrm{H}_{2} \mathrm{O}$ atmospheres. The $k$ data was first converted to surface exchange coefficient $\left(k_{s}\right)$ data using the following relationship derived in Appendix $\mathrm{C}$ : 


$$
k_{s}=\frac{1}{A} \frac{x_{v}^{e q b}}{\left(1-x_{v}^{e q b}\right)} k
$$

The $\mathrm{k}_{\mathrm{s}}$ data was converted to an exchange rate coefficient, $\mathfrak{R}_{0}$, to compare with the values extracted from our impedance measurements ${ }^{\dagger}$, using the following relationship [35]:

$\mathfrak{R}_{0}=k_{s} C_{0}$

The extracted $\mathfrak{R}_{0}$ values are a factor of 3 lower than values computed from Yashiro's $k$ data. This may be due to surface morphology differences, uncertainty in the value for $a$, or the difference in Gd doping levels. The slope of our data is approximately -0.25 , i.e. corresponding to $\mathfrak{R}_{0}$ proportional to $P_{\mathrm{O}_{2}}^{-0.25}$. The magnitude of the slope compares well with the +0.25 for Yashiro's $k$ data, although their value becomes approximately +0.2 when converted to $\mathfrak{R}_{0}$ values. However, the trend of increasing $\mathfrak{R}_{0}$ with respect to $P_{\mathrm{O}_{2}}$ observed by Yashiro is opposite to the data presented in this study. A literature survey revealed no additional exchange rate data for $\mathrm{CO}-\mathrm{CO}_{2}$ on ceria or other electrode/electrolyte materials.

\footnotetext{
${ }^{\dagger}$ Maier [52] discusses in detail a theoretical rationale for $k_{s}$ values being identical for isotope exchange and impedance experiments, and notes that experimental findings support this.
} 


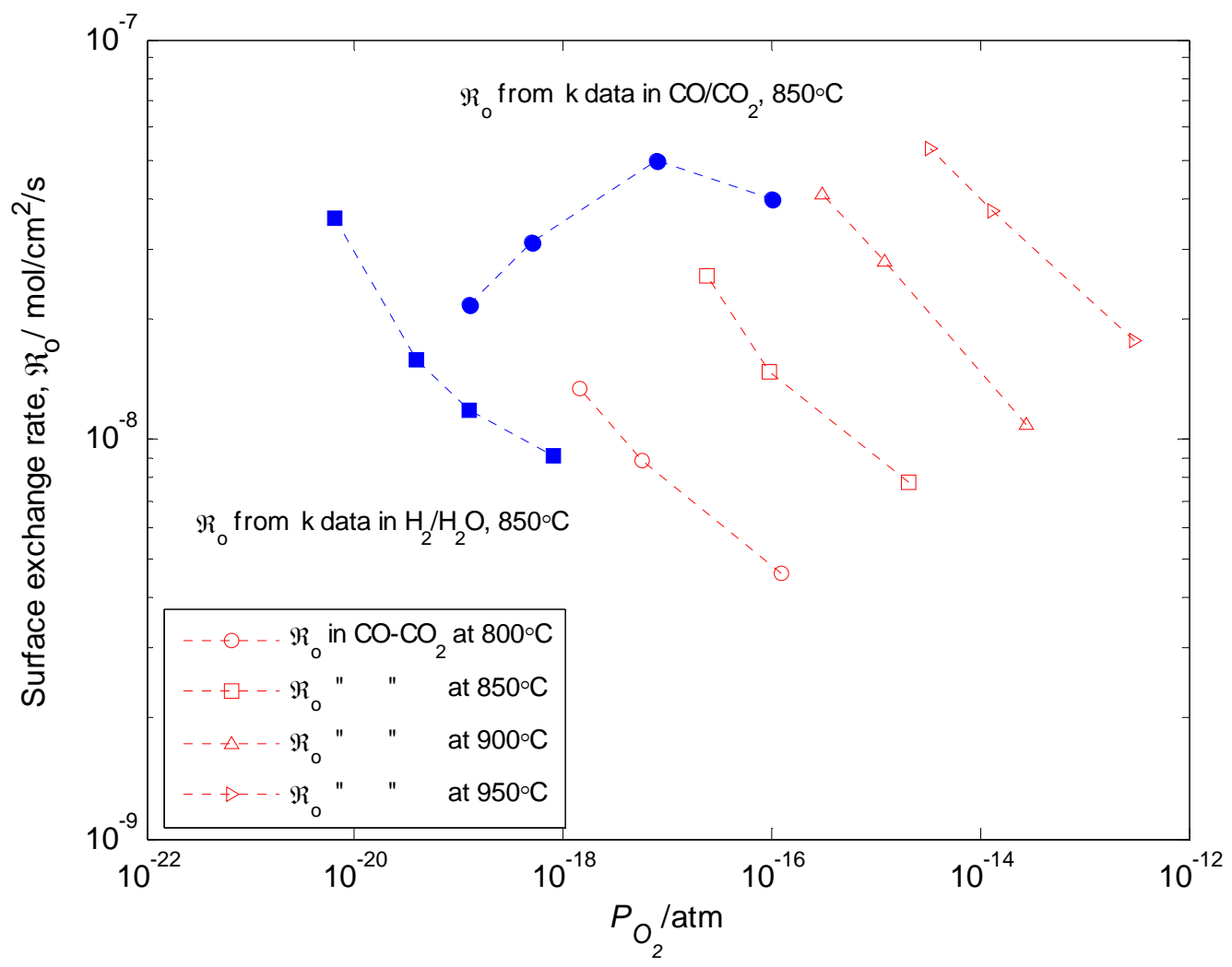

Figure 5-9. Exchange rate, $\mathfrak{R}_{0}$, extracted from impedance data for $\mathrm{CO} / \mathrm{CO}_{2}$ exchange on a $40 \mathrm{~mol} \%$ GDC electrode as a function of partial pressure of $\mathrm{O}_{2}$. Literature data (filled blue data points) are from surface rate coefficient data $(k)$ measured using thermogravimetry in ref. [32].

But, a direct comparison of the impedance results (i.e. $Z-R_{\text {ohmic }}$ ) with the impedance (or polarization) data from reference [22] on porous platinum electrodes and references [8,21] on Ni-YSZ electrodes, shows a decreasing ASR with increasing $\mathrm{CO} / \mathrm{CO}_{2}$ ratio (i.e. decreasing $P_{\mathrm{O}_{2}}$ ). This indicates these authors observed the same trend on porous Pt and Ni-YSZ electrodes as observed on GDC in this study (seen in Figure 55). An inspection of equations (5-1) and (5-2) indicates that the $\mathrm{CO}-\mathrm{CO}_{2}$ exchange 
model predicts that $\mathfrak{R}_{0} \propto \sqrt{\frac{1}{Z_{\text {chem }}}}$, confirming the trend if $\mathfrak{R}_{0}$ values were extracted from these authors' impedance results. As an aside, the reference [32] results for $\mathrm{H}_{2}-\mathrm{H}_{2} \mathrm{O}$ atmospheres are consistent with the surface exchange coefficient data on doped ceria reported by others $[33,36]$.

In contrast, Aaberg et. al. [37] do report a maximum open circuit admittance (i.e. proportional to $\mathfrak{R}_{0}$ ) at a $\mathrm{CO} / \mathrm{CO}_{2}$ ratio of 0.8 for impedance measurements on Ni-YSZ electrodes, but there is quite a bit of scatter in their data. And the $P_{\mathrm{O}_{2}}=10^{-14}$ atm corresponding to this $\mathrm{CO} / \mathrm{CO}_{2}$ ratio, is significantly higher than the $P_{\mathrm{O}_{2}}$ for an apparent maximum in $\mathfrak{R}_{0}$ calculated from the Yashiro et. al. [32] $k$ data.

Another possible explanation for the discrepancy in our results to those reported in reference [32], is the thermogravimetry measurement involves a large displacement in $P_{\mathrm{O}_{2}}$ from the equilibrium condition. This could mean that the kinetics, and in particular, the reaction mechanism may be different than for the small displacement from equilibrium made with an AC impedance measurement [38].

Finally, although $D_{v}$ and $A$ are expected to depend on $P_{\mathrm{O}_{2}}$ as a single independent variable, this does not indicate (i.e. implied in Figure 5-9) that $\mathfrak{R}_{0}$ only depends on $P_{\mathrm{O}_{2}}$. $\mathfrak{R}_{0}$ will depend on two variables, $P_{\mathrm{CO}}$ and $P_{\mathrm{CO}_{2}}$, so $\mathfrak{R}_{0}$ vs. $P_{c}\left(=P_{\mathrm{CO}}+P_{\mathrm{CO}_{2}}\right)$ would need 
to be measured as a second independent variable to fully map the kinetics of $\mathrm{CO}-\mathrm{CO}_{2}$ exchange on GDC electrodes.

\subsubsection{Comparison of GDC and Pt electrode impedance spectra}

Since platinum is a catalyst for $\mathrm{CO}$ oxidation, and as mentioned in the introduction, has been used as a cathode in previous carbon dioxide electrolysis work, it raises a question of whether the platinum mesh current collector used for the electrodes participates in the enhanced electrocatalytic activity observed on GDC. Park et al. [39] investigated hydrocarbon oxidation on ceria-based anodes and concluded that the $\mathrm{Pt}$ current collector contribution as a catalyst is insignificant. Tao and Irvine [40] performed impedance measurements of hydrogen oxidation on $\mathrm{Sc}_{0.15} \mathrm{Y}_{0.05} \mathrm{Zr}_{0.62} \mathrm{Ti}_{0.18} \mathrm{O}_{1.9}$ (or $\mathrm{ScYZT}$ ) anodes, along with Pt anodes and Pt current collector bounded to a porous YSZ interface, and concluded the Pt current collector (consisting of a porous Pt layer) does not enhance the performance of their ScYSZT anode. However, Marina and Mogensen [41] observed low electrocatalytic activity for methane oxidation on a GDC anode with a gold current collector, compared to GDC with a porous layer of Pt added as a current collector.

So, a separate experiment was performed by fabricating a porous Pt/YSZ/porous Pt cell, with the identical cell geometry and Pt mesh current collector used for the GDC cell. Figure 5-10 compares the resulting impedance measurements with the GDC cell results. The ASR of this Pt electrode cell was $\sim 663 \Omega-\mathrm{cm}^{2}$ at $800^{\circ} \mathrm{C}$ in $5 \% \mathrm{CO}$ in $\mathrm{CO}_{2}$, similar to impedance results on Pt electrodes reported by Mizusaki et al. [22]. This result 
is compared with the measured $\sim 3.8 \Omega-\mathrm{cm}^{2}$ for our GDC cell under identical conditions; the Pt electrode has an ASR two orders of magnitude higher, implying the Pt mesh in the GDC cell serves only as a current collector. The Pt mesh current collector bonded to the GDC could possibly act as a catalyst - as Pt/ceria is an excellent one for CO oxidation but this alone cannot explain such a large reduction in ASR, given the rather limited three-phase boundary area at the Pt mesh and porous GDC interface.

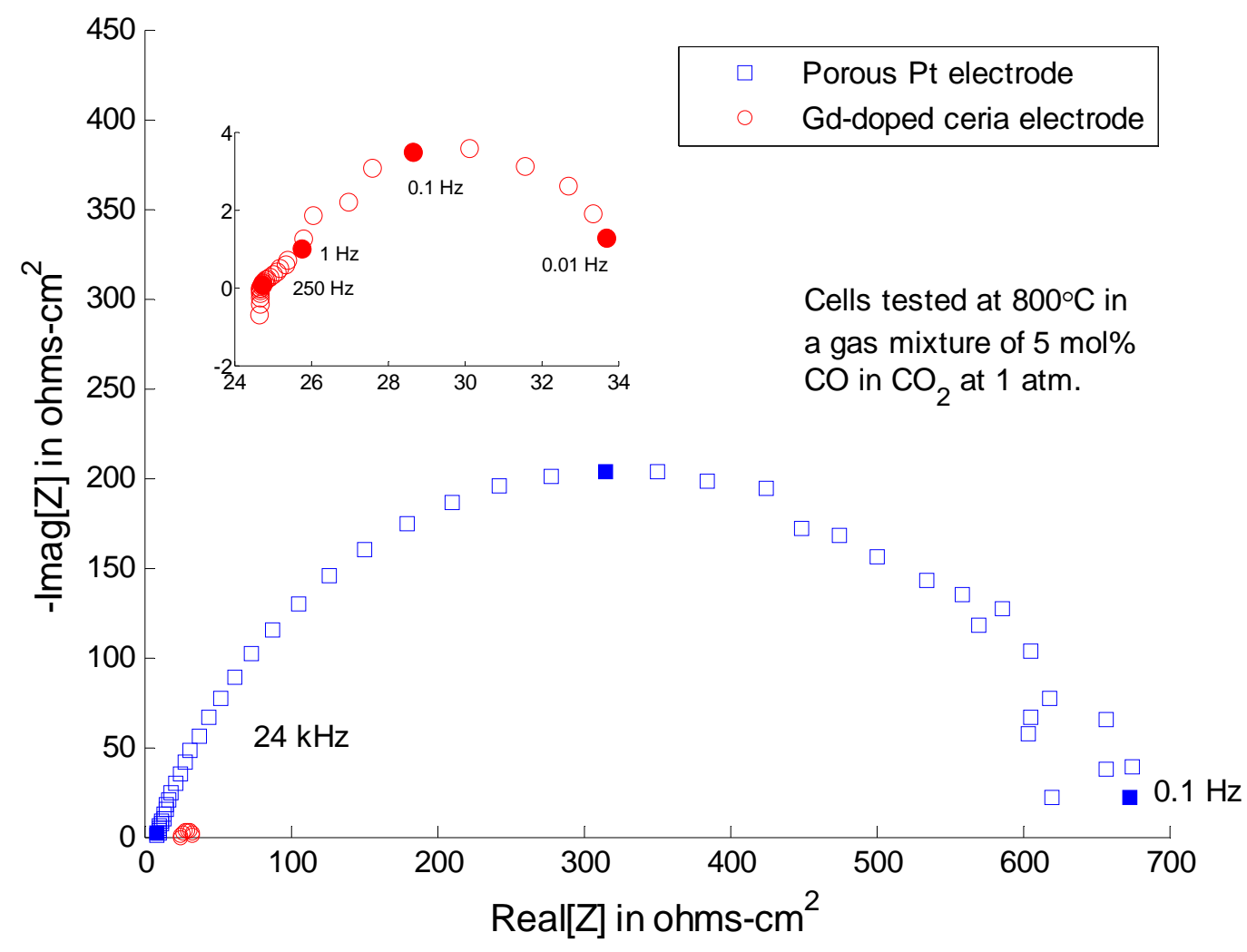

Figure 5-10. Comparison of impedance spectra of a Pt/YSZ/Pt cell with a GDC/YSZ/GDC cell. Cell geometry and test conditions are identical for both cells. Inset plot is an expanded scale view to show detail of GDC electrode spectra. 
And, the rather thick GDC electrode layer would limit oxygen ion transport and electronic conductivity (i.e. more so than a porous Pt electrode alone), if the large surface area/volume of the GDC electrode has little or no reactivity to $\mathrm{CO}_{2}$ reduction. Although the Pt from the current collector may have migrated into the GDC electrode via surface diffusion or vapor deposition during testing, Energy Dispersive Spectroscopy (EDS) performed as part of the SEM evaluation did not detect Pt in the interior of the electrode.

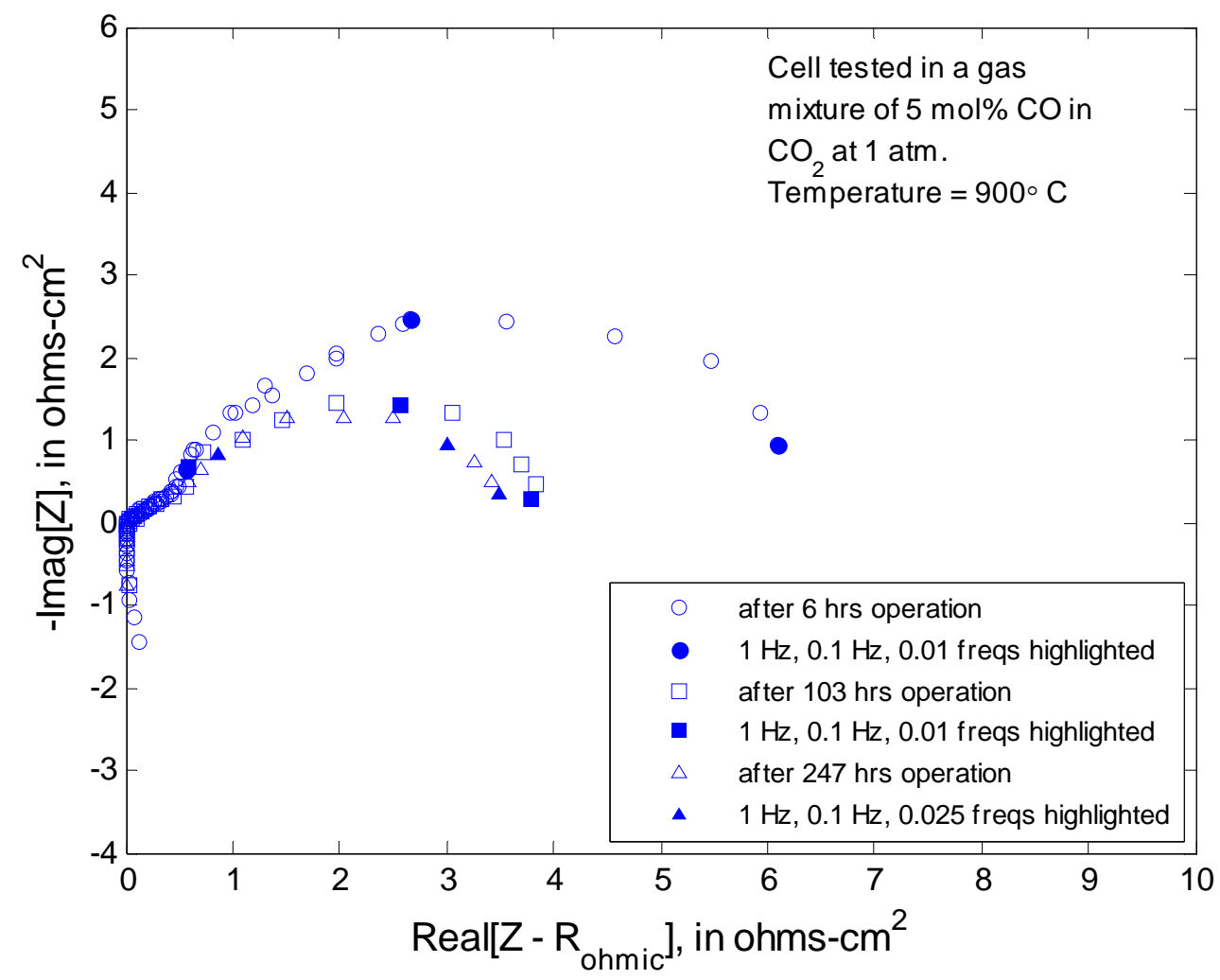

Figure 5-11. Change in impedance spectra with operational time. Cell was cycled through a temperature range of $700-950^{\circ} \mathrm{C}$ and $\mathrm{CO} / \mathrm{CO}_{2}$ gas mixture ratios of $0.05,0.20$, and 0.33 three or more times over this operational period. 


\subsubsection{GDC electrode performance vs. operational time}

The GDC electrode should be stable over time, as the ceria supports in catalytic converters operate in more severe and wider varying temperature and $P_{\mathrm{O}_{2}}$ conditions than our test conditions. However, other studies have observed material decomposition [6] in similar temperature and $P_{O_{2}}$ conditions, and reduced performance due to carbon deposition on Ni-YSZ electrodes in hydrocarbon mixtures [42]. The tested cell was operated for over 360 hours; over this time of operation, the complete test matrix was repeated $3+$ times to demonstrate repeatability. Figure 5-11 is a plot of impedance spectra at several points of operational time. The ASR decreased about $17 \%$ from initial measurements, and stabilized after approximately 100 hours of operation. After this period, the ASR varied less than $8 \%$ under all temperature and gas mixture conditions.

Using the same methodology discussed in the previous section, $D_{v}$ and $\mathfrak{R}_{0}$ were extracted from these 3 impedance spectra to see how the kinetics/diffusion phenomena are changing with time. The results indicate that $D_{v}$ essentially remains constant (0.2-0.4 $\times 10^{-7} \mathrm{~cm}^{2} / \mathrm{s}$ ), whereas $\mathfrak{R}_{0}$ increased an order of magnitude (from $0.6 \times 10^{-7}$ to $2.2 \times 10^{-7}$ $\mathrm{mol} / \mathrm{cm}^{2}$-s) during this time period, indicating that the kinetics, not solid-state diffusion, is improving with operational time. The utilization thickness ratio, $\phi$, also remains fairly constant (0.7-1.0) during this operational period, implying there is little change in the extent of activation of the electrode. Finally, the scatter in the impedance data around 1 $\mathrm{Hz}$ appears to decrease with time, resulting in improved fits to the $\mathrm{CO}-\mathrm{CO}_{2}$ exchange model for increased operational time. 


\subsubsection{Possible reasons for lower electrode ASR with operational time}

The decreasing ASR vs. operational time is intriguing, given that most solid oxide electrodes undergo a performance degradation (or increasing ASR) over time due to a variety of factors. In fact, the limited work on the aging behavior of GDC in the literature implies that degradation of GDC used as an electrode is likely. Zhang et al. [43] have reported a decrease in GDC ionic conductivity over a span of 3-8 days of operation at $1000^{\circ} \mathrm{C}$; they noted the degradation in ionic conductivity was more pronounced for higher dopant content $(x \geq 0.2)$ and attributed it to the formation of microdomains [44]. Also, Dikman et al. [31] looked at the stability of the $\mathrm{Gd}_{0.2} \mathrm{Ce}_{0.80} \mathrm{O}_{1.90}$ by annealing at $1000^{\circ} \mathrm{C}$ in air for a week and observed the separation of a secondary $\mathrm{Gd}_{2} \mathrm{O}_{3}$ phase [which would likely effect either ionic conductivity or surface reactivity].

As for potential reasons for the decrease in ASR, incomplete reduction of GDC can be eliminated. Undoped ceria rapidly becomes oxidized or reduced (i.e. time constant in seconds) in slightly oxidizing or reducing atmospheres (i.e. $P_{O_{2}}=10^{-3}-10^{-1}$ atm) when used as a support in automobile catalytic converters. For this experimental work, much larger changes in $P_{\mathrm{O}_{2}}$ were made but 10-12 hours were allowed for equilibration after each change in gas composition and 2 hours for each change in temperature. In dilatometry measurements made on $40 \mathrm{~mol} \%$ GDC, Mogensen and Mogensen [45] noted that the equilibrium was typically reached at 2-3 hours after similar 
changes in gas compositions, for dilatometry samples much larger in volume than our electrode. Finally, the measured $t_{\text {chem }}$ values - an indication of the kinetic response - are on the order of seconds, indicating that GDC electrode does indeed respond rapidly to the induced oxidation/reduction for small potential amplitude perturbations.

Carbon deposition is normally considered detrimental to performance in SOFC anodes, but other researchers $[46,47]$ have actually reported improved performance and reduced ASR for oxidation of methane and n-butane on $\mathrm{Cu}$-ceria electrodes and attribute part of this improvement to electronic conductivity enhancement from the carbon deposition they detected in the electrode. And, Marina et al. [48] have detected carbon deposition for methane oxidation on GDC anodes in low steam-to-methane gas mixture ratios, in as little as 100 hours of operation. At the $\mathrm{CO} / \mathrm{CO}_{2}$ ratios tested, carbon deposition is not thermodynamically favorable, but the limit is close enough that with the $\mathrm{AC}$ and polarization amplitudes induced on the cell, it is still prudent to check for this possibility. Optical microscopy, SEM, and EDS were performed on sectioned cells after completion of the testing, and no observable carbon deposition was detected. Although deposited carbon may exist below the detectable limit, such small amounts would not provide the connectivity between sintered GDC particles to provide any enhancement to electronic conductivity.

Another possible explanation is that the GDC electrode surface morphology is changing over time, possibly due to operating temperature. Increasing rates of $\mathrm{CO}$ 
oxidation have been reported on ceria powders calcined in air at successively higher temperatures [49] which are within the temperature range of operation for these cells.

\subsection{Conclusions}

In this chapter, initial $\mathrm{AC}$ impedance measurements for $\mathrm{CO}_{2}$ reduction on a 40 mol $\%$ gadolinium-doped ceria electrode were presented. The impedance measurements at zero-bias voltage indicate this electrode has a lower electrode area-specific-resistance (ASR) than for $\mathrm{CO}-\mathrm{CO}_{2}$ exchange on Ni-YSZ and porous platinum electrodes in the literature.

The impedance measurement data were fitted to the derived $\mathrm{CO}-\mathrm{CO}_{2}$ exchange model in Chapter 4, and the vacancy diffusion coefficient, $D_{v}$ and exchange rate, $\mathfrak{R}_{0}$, were extracted. The $D_{v}$ values correlated reasonably well when compared with data from IEDP-SIMS and conductivity measurements in the literature. On the other hand, the $\mathfrak{R}_{0}$ values obtained are a factor of 3 lower and increase with decreasing $P_{\mathrm{O}_{2}}$, a relationship opposite to the trend observed in the literature for $\mathrm{CO}-\mathrm{CO}_{2}$ exchange, but consistent with measurements for $\mathrm{H}_{2}-\mathrm{H}_{2} \mathrm{O}$ exchange on GDC. Finally, utilization thickness values, $\phi$, were extracted which suggest that the GDC electrode is transitioning from co-limited to kinetically limited behavior, implying that nearly the entire electrode thickness, $L$, is electro-catalytically active in the $\mathrm{T}=700-950^{\circ} \mathrm{C}$ and $P_{\mathrm{O}_{2}}=10^{-18}-10^{-14}$ atm conditions investigated. 


\section{$5.5 \quad$ Future Work}

We caution that limited work has been performed on solid oxide electrode materials under these particular $\mathrm{CO}-\mathrm{CO}_{2}$ conditions, as the primary research focus to date has been $\mathrm{CO}$ oxidation in mixtures of hydrogen and other hydrocarbons. Given the inconsistency in the $\mathfrak{R}_{0}$ results with those in the literature, it is recommended that future work include varying the total carbon pressure (i.e. $P_{\mathrm{CO}}+P_{\mathrm{CO}_{2}}$ ) to obtain a better understanding the $\mathrm{CO}-\mathrm{CO}_{2}$ exchange kinetics on GDC. Also, given that one of the extracted parameters, the utilization thickness, $\phi$, remained constant over the tested conditions, future work should include a study of either varying the electrode thickness or sintering temperature (i.e. vary electrode surface area, $a$ ) to properly validate the $\mathrm{CO}-\mathrm{CO}_{2}$ exchange model presented.

From a more applied research perspective, it is recommended that the addition of Pt or Rh to the ceria electrode, be investigated to assess if these noble metals - widely used to enhance $\mathrm{CO}$ oxidation in heterogeneous catalysis - could potentially improve $\mathrm{CO}_{2}$ reduction kinetics. And, because of the fabrication problems encountered in this research, it is recommended that some of the more recent fabrication methods, such as the aqueous impregnation of ceria into a porous YSZ substrate, be pursued to develop a more practical, durable $\mathrm{CO}_{2}$ reduction cathode. 


\subsection{Closing Remarks}

Our initial results documented in this work indicate that gadolinia-doped ceria is a promising material as a carbon dioxide reduction cathode, despite a number of challenges in working with this material for this particular application. We hope that further research in the solid oxide electrolysis of carbon dioxide will be galvanized by NASA's technology needs, along with the recent worldwide interest in the development of alternative fuels and reduction of carbon dioxide emissions.

\section{References:}

[1] SB Adler. Mechanism and kinetics of oxygen reduction on porous $\mathrm{La}_{1-\mathrm{x}} \mathrm{Sr}_{\mathrm{x}} \mathrm{CoO}_{3 \text {-delta }}$ electrodes, Solid State Ionics. 111 (1998) 125-134.

[2] C Gabrielli, Identification of electrochemical processes by frequency response analysis, 004/83 (1980) 3-6.

[3] AJ Bard, LR Faulkner, Electrochemical Methods: Fundamentals and Applications, second ed., Wiley, New York, NY, 2001. See Chapter 10 in this reference.

[4] JR Macdonald, WB Johnson, Fundamentals of Impedance Spectroscopy, in: Macdonald JR (Ed.), Impedance Spectroscopy, first ed., Wiley, New York, NY, 1987.

[5] W Lai, SM Haile. Impedance spectroscopy as a tool for chemical and electrochemical analysis of mixed conductors: A case study of ceria, J. Am. Ceram. Soc. 88 (2005) 29792997.

[6] G Lauvstad, R Tunold, S Sunde, Oxidation of carbon monoxide on strontium ferrate, 95-1 (1995) 731-740.

[7] P Holtappels, De Haart, L. G. J., U Stimming, IC Vinke, M Mogensen. Reaction of $\mathrm{CO} / \mathrm{CO} 2$ gas mixtures on Ni-YSZ cermet electrodes, J.Appl.Electrochem. 29 (1999) 561568. 
[8] Y Matsuzaki, I Yasuda. Electrochemical oxidation of $\mathrm{H} 2$ and $\mathrm{CO}$ in a $\mathrm{H}_{2}-\mathrm{H}_{2} \mathrm{O}-\mathrm{CO}-$ $\mathrm{CO}_{2}$ system at the interface of a Ni-YSZ cermet electrode and YSZ electrolyte, $\mathrm{J}$.

Electrochem. Soc. 147 (2000) 1630-1635.

[9] GO Lauvstad, R Tunold, S Sunde. Electrochemical oxidation of CO on Pt and Ni point electrodes in contact with an yttria-stabilized zirconia electrolyte - II. Steady-state and impedance measurements, J.Electrochem.Soc. 149 (2002) E506-E514.

[10] AM Sukeshini, B Habibzadeh, BP Becker, CA Stoltz, BW Eichhorn, GS Jackson. Electrochemical oxidation of $\mathrm{H}_{2}, \mathrm{CO}$, and $\mathrm{CO} / \mathrm{H}_{2}$ mixtures on patterned $\mathrm{Ni}$ anodes on YSZ electrolytes, J.Electrochem.Soc. 153 (2006) A705-A715.

[11] F Bidrawn, G Kim, G Corre, JTS Irvine, JM Vohs, RJ Gorte. Efficient Reduction of $\mathrm{CO}_{2}$ in a Solid Oxide Electrolyzer, Electrochem. Solid-State Lett. 11 (2008) B167-B170.

[12] W Weppner, Electrode performance, in: Bruce PG (Ed.), Solid State Electrochemistry, first ed., Cambridge University Press, Cambridge, UK, 1995, p. 204.

[13] A Constantinides, N Mostoufi, Numerical Methods for Chemical Engineers with MATLAB Applications, first ed., Prentice Hall PTR, Upper Saddle River, NJ, 1999.

[14] RJ Sheppard, BP Jordan, EH Grant. Least squares analysis of complex data with applications to permittivity measurements, J. Phys. D: Appl. Phys. 3 (1970) 1759-1764.

[15] M Mogensen, Ceria-based electrodes, in: Trovarelli A (Ed.), Catalysis by Ceria and Related Materials, vol. 2, Imperial College Press, London, 2002, pp. 453-481.

[16] EE Underwood, Quantitative Stereology, Addison-Wesley, Reading, MA, 1970.

[17] JR Wilson, W Worawarit, R Mendoza, HY Chen, JM Hiller, DJ Miller, et al. Threedimensional reconstruction of a solid-oxide fuel-cell anode, Nature Materials. 5 (2006) 541-544.

[18] RJ Gorte, S Park, JM Vohs, C Wang. Anodes for direct oxidation of dry hydrocarbons in a solid-oxide fuel cell, Adv. Mater. 12 (2000) 1465-1469.

[19] BCH Steele, KM Hori, S Uchino. Kinetic parameters influencing the performance of IT-SOFC composite electrodes, Solid State Ionics. 135 (2000) 445-450.

[20] T Nakamura, T Kobayashi, K Yashiro, A Kaimai, T Otake, K Sato, et al.

Electrochemical behaviors of mixed conducting oxide anodes for solid oxide fuel cell, $\mathrm{J}$. Electrochem. Soc. 6 (2008) B563-B569.

[21] FZ Boulenouar, K Yashiro, M Oishi, A Kaimai, Y Nigara, T Kawada, et al., Electrochemical oxidation of $\mathrm{CO}$ in a $\mathrm{CO}-\mathrm{CO}_{2}$ system at the interface of Ni grid electrode/YSZ electrolyte, in: Yokokawa H, Singhal SC (Eds.), Proceedings of the Seventh International Symposium on Solid Oxide Fuel Cells, SOFC VII, PV2001-16, Pennington, NJ ed., The Electrochemical Society, Pennington, NJ, 2001, pp. 759-768. 
[22] J Mizusaki, H Tagawa, Y Miyaki, S Yamauchi, K Fueki, I Koshiro, et al. Kinetics of the Electrode-Reaction at the $\mathrm{CO}-\mathrm{CO}_{2}$, Porous Pt/stabilized Zirconia Interface, Solid State Ionics. 53-56 (1992) 126-134.

[23] JJ Sprague, O Porat, HL Tuller. Mixed conducting gas sensors: atmosphere dependent electrode impedance, Sensors and Actuators B. 35 (1996) 348-352.

[24] M Breysse, M Guenin, B Claudel, H Latreille, J Veron. Catalysis of carbon monoxide by cerium dioxide I. Correlations between catalytic activity and electrical conductivity, J. Catalysis. 27 (1972) 275-280.

[25] MF Wilkes, P Hayden, AK Bhattacharya. Catalytic studies on ceria lanthana solid solutions II. Oxidation of carbon monoxide, J. Catalysis. 219 (2003) 295-304.

[26] Z Shao, SM Haile. A high-performance cathode for the next generation of solidoxide fuel cells, Nature. 431 (2004) 170-173.

[27] B Zachau-Christianson, T Jacobsen, K West, S Skaarup, Electrochemical determination of the oxygen stoichiometry of doped ceria, in: Singhal SC, Iwahara $\mathrm{H}$ (Eds.), Proceedings of the Third International Symposium on Solid Oxide Fuel Cells, SOFC III, PV93-4, Pennington, NJ ed., The Electrochemical Society, Pennington, NJ, 1993, pp. 104-111.

[28] PS Manning, JD Sirman, JA Kilner. Oxygen self-diffusion and surface exchange studies of oxide electrolytes having the fluorite structure, Solid State Ionics. 93 (1996) 125-132.

[29] D Perez-Coll, P Nunez, JC Ruiz-Morales, J Pena-Martinez, JR Frade. Reexamination of bulk and grain boundary conductivities of $\mathrm{Ce}_{1-\mathrm{x}} \mathrm{Gd}_{\mathrm{x}} \mathrm{O}_{2 \text {-delta }}$ ceramics, Electrochim. Acta. 52 (2007) 2001.

[30] Bouwmeester, H. J. M., AJ Burggraaf, Dense membranes for oxygen separation, in: Gellings PJ, Bouwmeester, H. J. M. (Eds.), The CRC Handbook of Solid State Electrochemistry, CRC Press, New York, 1997, p. 503.

[31] S Dikmen, P Shuk, M Greenblatt, H Gocmez. Hydrothermal synthesis and properties of $\mathrm{Ce}_{1-\mathrm{x}} \mathrm{Gd}_{\mathrm{x}} \mathrm{O}_{2 \text {-delta }}$ solid solutions, Solid State Sciences. 4 (2002) 585-590.

[32] K Yashiro, S Onuma, A Kaimai, Y Nigara, T Kawada, J Mizusaki, et al. Mass transport properties of $\mathrm{Ce}_{0.9} \mathrm{Gd}_{0.1} \mathrm{O}_{2 \text {-delta }}$ at the surface and in the bulk, Solid State Ionics. 152-153 (2002) 469-476.

[33] JA Lane, JA Kilner. Oxygen surface exchange on gadolinia doped ceria, Solid State Ionics. 136-137 (2000) 927-932.

[34] JA Kilner. Fast oxygen transport in acceptor doped oxides, Solid State Ionics. 129 (2000) 13-23. 
[35] SB Adler, JA Lane, BCH Steele. Electrode kinetics of porous mixed-conducting oxygen electrodes, J.Electrochem.Soc. 143 (1996) 3554-3564.

[36] T Horita, K Yamaji, N Sakai, M Ishikawa, H Yokokawa, T Kawada, et al. Oxygen surface exchange of $\mathrm{Y}_{0.2} \mathrm{Ce}_{0.8} \mathrm{O}_{2-\mathrm{x}}$ under reducing atmosphere, Electrochem. Solid-State Lett. 1 (1998) 4-6.

[37] RJ Aaberg, R Tunold, S Tjelle, R Odegard, Oxidation of $\mathrm{CO}$ and $\mathrm{H}_{2}$ on Ni/YSZ cermet electrodes, in: Poulsen FW, Bonanos N, Linderoth S, Mogensen M, ZachauChristiansen B (Eds.), High Temperature Electrochemistry: Ceramics and Metals, Proceedings of the 17th Riso International Symposium of Materials Science, Roskilde, Denmark ed., Riso National Laboratory, Roskilde, Denmark, 1996, pp. 511-516.

[38] SB Adler, XY Chen, JR Wilson. Mechanisms and rate laws for oxygen exchange on mixed-conducting oxide surfaces, J. Catalysis. 245 (2007) 91-109.

[39] S Park, JM Vohs, RJ Gorte. Direct oxidation of hydrocarbons in a solid-oxide fuel cell, Nature. 404 (2000) 265-267.

[40] S Tao, Irvine, J. T. S. Investigation of the mixed conducting oxide ScYZT as a potential SOFC material, J. Electrochem. Soc. 151 (2004) A497-A503.

[41] OA Marina, M Mogensen. High temperature conversion of methane on a composite gadolinia-doped ceria-gold electrode, Appl. Catal. A. 189 (1999) 117-126.

[42] T Takeguchi, Y Kani, T Yano, R Kikuchi, K Eguchi, K Tsujimoto, et al. Study on steam reforming of $\mathrm{CH}_{4}$ and $\mathrm{C}_{2}$ hydrocarbons and carbon deposition on Ni-YSZ cermets, J. Power Sources. 112 (2002) 588.

[43] TS Zhang, J Ma, LB Kong, SH Chan, JA Kilner. Aging behavior and ionic conductivity of ceria-based ceramics: a comparative study, Solid State Ionics. 170 (2004) 209-217.

[44] T Mori, J Drennan, Y Wang, JH Lee, Jg Li, T Ikegami. Electrolytic Properties and Nanostructural Features in the $\mathrm{La}_{2} \mathrm{O}_{3}-\mathrm{CeO}_{2}$ System, J. Electrochem. Soc. 150 (2003) A665-A673.

[45] G Mogensen, M Mogensen. Reduction Reactions in Doped Ceria Ceramics Studied by Dilatometry, Thermochimica Acta. 214 (1993) 47-50.

[46] H Kim, C Lu, WL Worrell, JM Vohs, RJ Gorte. Cu-Ni cermet anodes for direct oxidation of methane in solid-oxide fuel cells, J.Electrochem.Soc. 149 (2002) A247A250.

[47] S McIntosh, JM Vohs, RJ Gorte. Role of hydrocarbon deposits in the enhanced performance of direct-oxidation SOFCs, J.Electrochem.Soc. 150 (2003) A470-A476. 
[48] OA Marina, C Bagger, S Primdahl, M Mogensen. A solid oxide fuel cell with a gadolinia-doped ceria anode: preparation and performance, Solid State Ionics. 123 (1999) 199-208.

[49] E Aneggi, J Llorca, M Boaro, A Trovarelli. Surface-structure sensitivity of CO oxidation over polycrystalline ceria powders, J. Catal. 234 (2005) 88-95.

[50] PW Atkins, Physical Chemistry, first ed., W. H. Freeman and Company, San Francisco, CA, 1978, p. 840.

[51] RJ Borg, GJ Dienes, An Introduction to Solid State Diffusion, first ed., Academic Press, San Diego, CA, 1988, p. 72.

[52] J Maier. On the correlation of macroscopic and microscopic rate constants in solid state chemistry, Solid State Ionics. 112 (1998) 197-228. 


\section{Appendix A}

\section{IMPLEMENTATION OF THE PELLET GEOMETRY MODEL IN THE FINITE ELEMENT ANALYSIS PACKAGE, COMSOL MULTIPHYSICS ${ }^{\circledR}$}

Although today's mathematical and engineering software have become more powerful in function and easier to use with well-designed graphical user interfaces (GUI's), the implementation and solution of a model is not necessarily straightforward. In this appendix, a description of the details is provided for implementing the reference electrode placement analyses model in Chapter 2, into the finite element analysis package, COMSOL Multiphysics.

\section{A.1 Model geometry}

Implementation of the model geometry can be performed with either text-based input or GUI geometry editor. Use of the GUI commands to construct simple 2D geometry model, e.g. Figure 2-1, is fairly straightforward. Alternatively, for simple geometries like this, COMSOL also allows one to construct a geometry using text-based line input, such as the following:

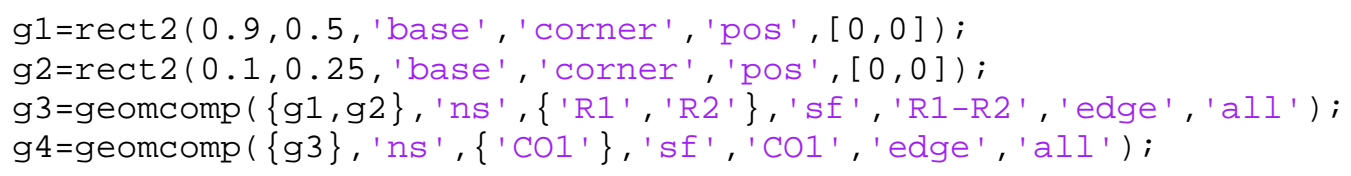

The commands above will generate the simple 2D pellet geometry model for a $0.9 \mathrm{~cm}$ radius, $0.5 \mathrm{~cm}$ height pellet with a hole $0.1 \mathrm{~cm}$ in diameter and $0.25 \mathrm{~cm}$ in depth. Key in implementation of the FEM model geometry is 1) making sure the subdomain is 
completely bounded, and 2) cleverly utilizing the Union, Intersection, and Difference functions to join, take the intersection, or remove sections of the drawn geometry to achieve the desired domain.

\section{A.2 Laplace's equation in the computational subdomain}

The PDE coefficient form of the equation is applied over the bounded computational domain, $\Omega[1]$ :

$$
\nabla \cdot(-c \nabla u-a u+\gamma)+\mathrm{a} u+\beta \cdot \nabla u=f
$$

where $u=$ dependent variable (= electrolyte potential, V)

$a$, a, $c, \beta$, and $f$ are complex valued scalar functions defined on $\Omega$.

To implement the 2D axisymmetric model, the coefficients in Equation (A-1) are set as follows:

$$
a=\mathrm{a}=f=\beta=\gamma=0 ; c=k_{\text {electrolyte }} \cdot x
$$

$$
\begin{aligned}
& \text { where } x=\text { the radial coordinate independent variable } \\
& \text { for the pellet geometry model } \\
& k_{\text {electrolyte }}=\text { YSZ electrolyte conductivity, S/cm }
\end{aligned}
$$

Thus, Laplace's equation in an axisymmetric geometry is implemented.

\section{A.3 Boundary conditions}

In COMSOL, two types of boundary conditions can be applied:

1. A generalized Neumann boundary condition defined as:

$$
\mathbf{n} \cdot(c \nabla u+a u-\gamma)+q u=g \text { on } \partial \Omega
$$

2. A Dirichlet boundary condition defined as:

$$
h u=r \text { on } \partial \Omega
$$


where $\mathbf{n}$ is the outward normal vector of the boundary surface $\partial \Omega$ is the boundary (surface) of interest

$g, q, h$, and $r$ are complex valued functions defined on $\partial \Omega$

For the pellet geometry model, the boundary conditions for the electrode surfaces are not a flux-type conditions, and the other boundary surfaces are insulating or zero-flux boundary conditions. Therefore, the PDE coefficient form for a Dirichlet boundary condition, $\partial \Omega$, is utilized:

$$
\mathbf{n} \cdot(c \nabla u+a u-\gamma)+q u=g-h^{T} ; h u=r
$$

where $\mathrm{g}$ and $\mathrm{q}$ are coefficients, and $\mathrm{h}=$ ?

For the working electrode boundary condition, the coefficients are set as follows:

$$
\begin{aligned}
& q=\frac{x\left(1-j \sigma \mathrm{R}_{\mathrm{WE}} \mathrm{C}_{\mathrm{WE}}\right)}{\mathrm{R}_{\mathrm{WE}}} \\
& g=h=r=0
\end{aligned}
$$

For the counter electrode boundary condition, the coefficients are set as follows:

$$
\begin{aligned}
& q=\frac{\mathrm{x}\left(1-j \sigma \mathrm{R}_{\mathrm{CE}} \mathrm{C}_{\mathrm{CE}}\right)}{\mathrm{R}_{\mathrm{CE}}} \\
& g=\frac{-10 \mathrm{x}\left(1-j \sigma \mathrm{R}_{\mathrm{CE}} \mathrm{C}_{\mathrm{CE}}\right)}{\mathrm{R}_{\mathrm{CE}}} \\
& h=r=0
\end{aligned}
$$

The -10 value in the equation for $\mathrm{g}$ is to set the counter-electrode potential to $-10 \mathrm{mV}$. The remaining surfaces in the model are insulated, and the coefficients of Equation (A-5) are set as follows:

$$
q=g=h=r=0
$$

These set coefficients listed in (A-6) through (A-8) translate the boundary conditions defined in Equation (2-13) into the PDE coefficient form as defined in the COMSOL 
Multiphysics software. Note in particular, the radial coordinate variable, $x$, is multiplied through in order to match the input format for the PDE coefficient form to implement in

the model. The other reason is that inputting a $\frac{1}{r}$ coefficient in the $\frac{\partial \Phi}{\partial r}$ term would cause numerical problems due to the singularity at the $r=0$ boundary in the axisymmetric model.

\section{Reference:}

[1] COMSOL AB, COMSOL Multiphysics Modeling Guide, version 3.2 (2005) 225-229. 


\section{Appendix B}

\section{DESCRIPTION AND CALIBRATION OF OXYGEN SENSOR TO MEASURE $\mathrm{CO} / \mathrm{CO}_{2}$ GAS MIXTURE RATIOS}

As discussed in Chapter 3, a Pt/YSZ/Pt oxygen sensor was fabricated and used to monitor $P_{\mathrm{O}_{2}}$ conditions during experimental testing of the GDC cells in CO-CO atmospheres in this work. The Pt/YSZ/Pt oxygen sensor was fabricated from a 7/16 in. (11.1 mm) OD closed-one-end (COE) tube of 15 mol\% YSZ. Pt ink was brush painted on the inside and outside closed end of the tube and fired under the identical conditions for the Pt/YSZ/Pt cells described in Chapter 3. A Swaglelok UltraTorr fitting was used to seal the open end and supply gas to the Pt electrodes inside the YSZ tube while the outside Pt sensor was exposed to air. The sensor was mounted in a small tube furnace.

The $\mathrm{O}_{2}$ sensor was calibrated using three gas mixtures, 2.5\% CO/97.5\% $\mathrm{CO}_{2}, 5 \%$ $\mathrm{CO} / 95 \% \mathrm{CO}_{2}$, and $10 \% \mathrm{CO} / 90 \% \mathrm{CO}_{2}$. The calibration was performed in the same gas flow system setup in Figure 3-6, but the alumina tube test chamber was bypassed. Gas flow rates of 30-100 sccm were used and the flow was varied until the measured sensor potential was stable with flow rate. The sensor furnace temperature was varied from $500^{\circ}$ to $900^{\circ} \mathrm{C}$.

The theoretical Nernst potential for the sensor was calculated using the following relationship: 


$$
E=E^{0}-\frac{R T}{2 F} \ln \frac{y_{c o}}{y_{\mathrm{O}_{2}}^{1 / 2}\left(1-y_{\mathrm{CO}_{2}}\right)} \text { where } E^{0}=-\frac{\Delta G_{\mathrm{CO}_{2}}^{0}}{2 F}
$$

The change in Gibbs free energy, $\Delta G_{\mathrm{CO}_{2}}^{0}$, was calculated from free energies of formation thermodynamic data in the NIST-JANAF tables. The $\mathrm{O}_{2}$ sensor potential data and calculated Nernst potential are displayed in Figure B-1.

The calculated Nernst potential is $\sim 20-30 \mathrm{mV}$ lower than the measured potential at $700^{\circ} \mathrm{C}$ and increases to $40-50 \mathrm{mV}$ at $900^{\circ} \mathrm{C}$. This is most likely due to the sensor temperature being lower than the temperature set point of the furnace used to heat the sensor, i.e. a thermocouple was not mounted on or near the $\mathrm{O}_{2}$ sensor. This offset was not problematic as the gas mixtures used for testing were pre-mixed by the supplier so the $\mathrm{O}_{2}$ sensor's main function was to monitor the $P_{\mathrm{O}_{2}}$ condition to verify no leaks in the gas flow system, etc.

As discussed in Chapter 3, the $\mathrm{O}_{2}$ sensor was mounted downstream of the test chamber. It should be noted that Darken and Gurry [1] and Huebner [2] have both reported in detail on the difficulties of measuring $P_{\mathrm{O}_{2}}$ in highly reducing atmospheres and recommended that the $\mathrm{O}_{2}$ sensor be placed as close to the sample (or cell) being measured. Unfortunately, the practical considerations for our test setup, typical of many SOFC researchers, do not make it feasible to position an $\mathrm{O}_{2}$ sensor of this type in the same chamber as the experimental test cell. 


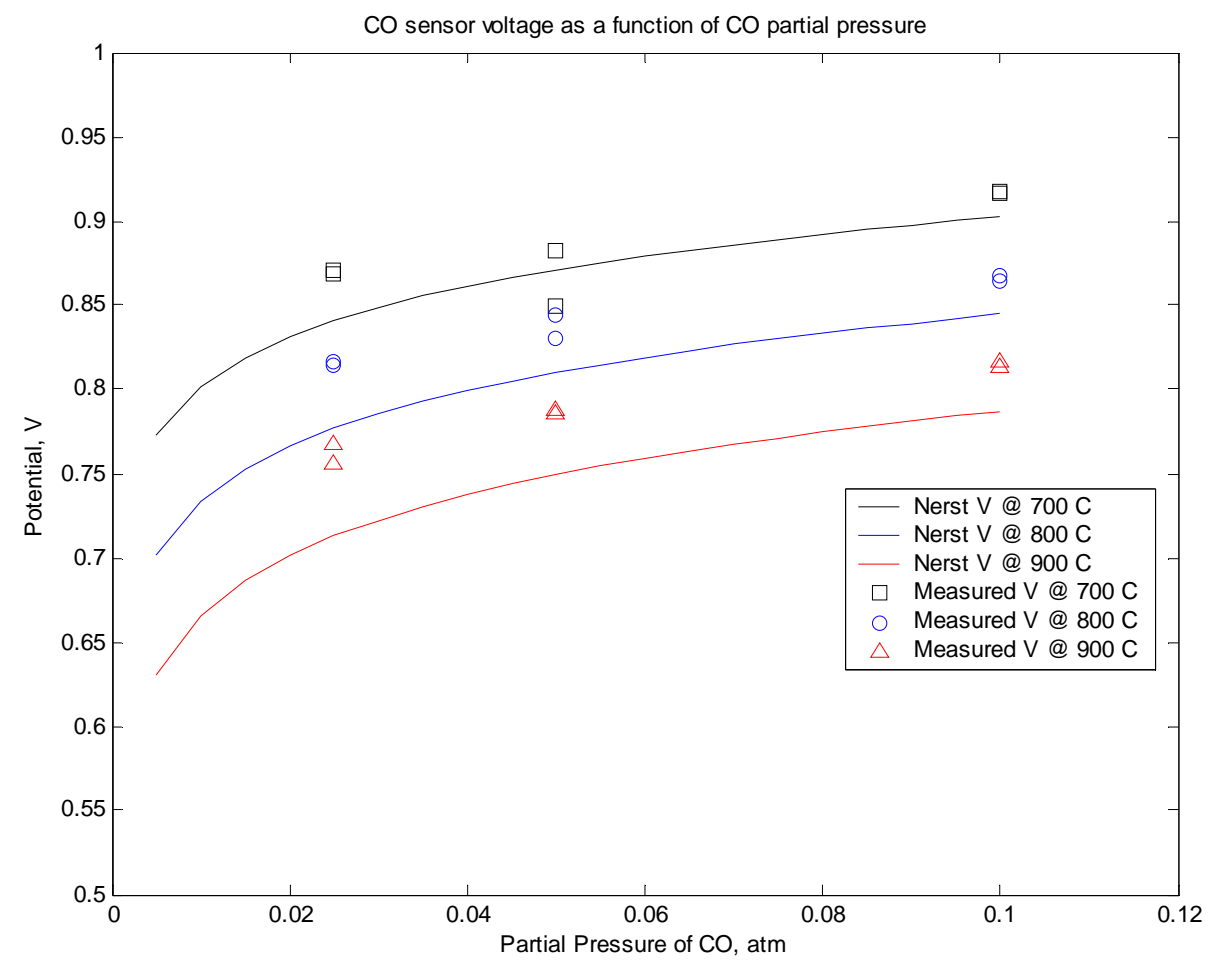

Figure B-1. Pt/YSZ/Pt oxygen sensor potential measurements made for calibration.

\section{References:}

[1] LS Darken, RW Gurry. The system iron-oxygen. I. The Wustite field and related equilibria, J. Amer. Chem. Soc. 67 (1945) 1398-1411.

[2] JS Huebner. Oxygen fugacity values of furnace gas mixtures, Am. Mineral. 60 (1975) 815-823. 


\section{Appendix C}

\section{DERIVATION OF THE RELATIONSHIP BETWEEN THE SURFACE REACTION RATE CONSTANT, $k$, AND SURFACE EXCHANGE COEFFICIENT, $k_{s}$}

In order to compare the exchange rate $\left(\mathfrak{R}_{O}\right)$ data extracted from our impedance measurements in Chapter 5 with published surface reaction rate constant $(k)$ data in the literature, we need to convert the $k$ values to surface exchange coefficient $\left(k_{s}\right)$ values.

The surface reaction rate constant, is defined as the flux at the surface of the solid for an abrupt displacement of the concentration from equilibrium, as done for a thermogravimetry experiment:

$$
\left.j_{O}\right|_{\text {at surface }}=k\left[\left.c_{o}(t)\right|_{\text {at surface }}-\left.c_{o}(\infty)\right|_{\text {at surface }}\right]
$$

$\left.c_{O}(t)\right|_{\text {at surface }}$ is the oxygen concentration just inside the surface of the solid) at any time, $t$, during the experiment and $\left.c_{O}(\infty)\right|_{\text {at surface }}$ is the oxygen concentration just inside the surface that is in equilibrium with the atmosphere of the thermogravimetry test chamber. An alternative description to Equation (C-1) is a surface boundary condition that assumes first order kinetics for the $\mathrm{CO}-\mathrm{CO}_{2}$ exchange occurring on this surface. This boundary condition is applied to Fick's Second Law to solve for the change in mass vs. time of a test sample in a thermogravimetric experiment. Katsuki et al. [1] provides an analytical 
solution for the commonly used rod or cylinder-shaped test sample, derived from the plane sheet and long cylinder solutions in Crank [2]. Yasuda and Hikita [3] provide a similar solution applied to the electrical conductivity relaxation technique.

For a small oxygen chemical potential gradient, the oxygen flux defined in Equation (C-1) may also be expressed in terms of Onsagar's equation[4]:

$$
\left.j_{O}\right|_{\text {at surface }}=\left.j_{e x}\right|_{\text {at surface }} \frac{\Delta \mu_{O}}{R T}
$$

where $j_{e x}$ is the flux of oxygen at the equilibrium condition and $\Delta \mu_{O}$ is the gradient of the oxygen chemical potential.

The equilibrium oxygen flux, $j_{e x}$, can be expressed as:

$$
j_{e x}=k_{s} c_{O}
$$

where $k_{\mathrm{s}}$ is the surface exchange coefficient.

Equation (C-3) provides the definition for the surface exchange coefficient, $k_{s}$. Combining equations (C-2) and (C-3), and converting to differential form:

$$
k=k_{s} \frac{c_{O}}{R T} \frac{\partial \mu_{O}}{\partial c_{O}}
$$


This implicitly assumes that the order of the exchange kinetics does not change. Equation (C-4) can be expressed in terms of the mole fraction of the vacancy concentration, $x_{v}$, by noting that $c_{O}=c_{m c}-x_{v} c_{m c}$, where $c_{m c}$ is the total concentration of oxygen lattice sites in the mixed conductor. Utilizing this expression, Equation (C-4) becomes:

$$
k=k_{s} \frac{-\left(1-x_{v}\right)}{R T} \frac{\partial \mu_{O}}{\partial x_{v}}
$$

The equilibrium exchange of oxygen occurring on the internal surface area of the GDC electrode, in Kröger-Vink notation [5], is:

$$
\frac{1}{2} O_{2}+V_{O}^{\bullet \bullet}+2 e^{\prime} \rightleftharpoons O_{O}^{x}
$$

The resulting equilibrium condition for (C-6) is:

$$
\frac{1}{2} \mu_{O_{2}}+\mu_{v}+2 \mu_{e}=\mu_{O}
$$

Note that $\mu_{O_{0}^{x}}$ can be neglected if $\mu_{v_{o}^{*}}$ is treated as the chemical potential of a vacancy building unit [6].

Expressing Equation (C-7) in differential form,

$$
\frac{1}{2} \partial \mu_{O_{2}}=\partial \mu_{O}
$$


$\partial \mu_{e^{\prime}}$ can be neglected as the concentration of electrons remains relatively constant for small displacements in $x_{v}[7,8]$. Equation (C-8) can be substituted into (C-5) to obtain:

$$
k=k_{s} \frac{-\left(1-x_{v}\right)}{2 R T} \frac{\partial \mu_{O_{2}}}{\partial x_{v}}
$$

The oxygen chemical potential is defined as:

$$
\mu_{\mathrm{O}_{2}}^{\text {solid }}=\mu_{\mathrm{O}_{2}}^{0}+R T \ln a_{\mathrm{O}_{2}}^{\text {solid }}=\mu_{\mathrm{O}_{2}}^{0}+R T \ln \frac{f_{\mathrm{O}_{2}}^{\text {solid }}}{f_{\mathrm{O}_{2}}^{0}}
$$

$\mu_{\mathrm{O}_{2}}^{0}$ is the standard or reference state of the oxygen chemical potential, $a_{\mathrm{O}_{2}}^{\text {solid }}$ is the activity of oxygen gas in the solid.

$f_{\mathrm{O}_{2}}$ is the oxygen fugacity in the solid.

$f_{\mathrm{O}_{2}}^{0}$ is the fugacity of the pure oxygen gas at the reference state, $=1 \mathrm{~atm}$.

Substituting the differential form of $\mu_{\mathrm{O}_{2}}$ into Equation (C-9) and also noting that if $P_{\mathrm{O}_{2}}$ is in equilibrium with the GDC solid, i.e. $P_{\mathrm{O}_{2}}=f_{\mathrm{O}_{2}}^{\text {solid }}$, then:

$$
k=k_{s} \frac{-\left(1-x_{v}\right)}{2} \frac{\partial \ln P_{O_{2}}}{\partial x_{v}}
$$

Applying the definition of the differential form $\left(\partial x_{v}=x_{v} \partial \ln x_{v}\right)$ :

$$
k=-\frac{1}{2} k_{s} \frac{\left(1-x_{v}\right)}{x_{v}} \frac{\partial \ln P_{\mathrm{O}_{2}}}{\partial \ln x_{v}}
$$


Utilizing our definition of $A\left(=-\frac{1}{2} \frac{\partial \ln P_{\mathrm{O}_{2}}}{\partial \ln x_{v}}\right)$, Equation (C-12) can be rewritten as:

$$
k_{s}=\frac{1}{A} \frac{x_{v}^{e q b}}{\left(1-x_{v}^{e q b}\right)} k
$$

This is the relationship given in Equation (5-11) in Chapter 5.

\section{References:}

[1] M Katsuki, S Wang, K Yasumoto, M Dokiya. The oxygen transport in Gd-doped ceria, Solid State Ionics. 154-155 (2002) 589-595.

[2] J Crank, Chapter 5, The Mathematics of Diffusion, second ed., Oxford University Press, New York, NY, 1975, pp. 79-80.

[3] I Yasuda, T Hikita. Precise determination of the chemical diffusion coefficient of calcium-doped lanthanum chromites by means of electrical conductivity relaxation, J. Electrochem. Soc. 141 (1994) 1268-1273.

[4] K Yashiro, S Onuma, A Kaimai, Y Nigara, T Kawada, J Mizusaki, et al. Mass transport properties of $\mathrm{Ce}_{0.9} \mathrm{Gd}_{0.1} \mathrm{O}_{2 \text {-delta }}$ at the surface and in the bulk, Solid State Ionics. 152-153 (2002) 469-476.

[5] FA Kroger, HJ Vink, Relations between the concentrations of imperfections in crystalline solids, in: Seitz F, Turnull D (Eds.), Solid State Physics - Advances in Research and Applications, Academic Press, New York, NY, 1956, pp. 431-435.

[6] Lankhorst, M. H. R., Bouwmeester, H. J. M., H Verweij. Use of the rigid band formalism to interpret the relationship between $\mathrm{O}$ chemical potential and electron concentration in $\mathrm{La}_{1-\mathrm{x}} \mathrm{Sr}_{\mathrm{x}} \mathrm{CoO}_{3 \text {-delta, }}$ Phys. Rev. Lett. 77 (1996) 2989-2992.

[7] E Ruiz-Trejo, J Maier. Electronic transport in single crystals of Gd-doped ceria, J. Electrochem. Soc. 154 (2007) B583-B587.

[8] I Riess, Electrochemistry of Mixed Ionic-Electronic Conductors (chapter 7), The CRC Handbook of Solid State Electrochemistry, first edition ed., CRC Press, Boca Raton, FL, 1997, pp. 223-268. 


\section{Appendix D}

\section{DERIVATION OF THE GDC OXYGEN NON-STOICHIOMETRY \\ MODEL AND THE RESULTING RELATIONSHIP FOR THE \\ THERMODYNAMIC FACTOR, $A$}

In this appendix, the thermodynamic factor relationship used in Equation (5-8) is derived, along with the relationship used in Equation (5-7) to fit oxygen nonstoichiometry data for GDC. The derivation of the oxygen nonstoichiometric relationship using ideal defect theory was presented by Yashiro et al. [1] ${ }^{*}$, but there appears to be a small error in the derivation, which is corrected in the derivation here.

\section{D.1 Derivation of the GDC oxygen non-stoichiometry model}

The defect reaction for oxygen vacancy formation in GDC can be expressed as:

$$
2 C e_{C e}^{x}+O_{O}^{x}=2 C e_{C e}^{\prime}+V_{O}^{\bullet \bullet}+\frac{1}{2} O_{2}(g)
$$

The equilibrium constant, $K_{e q}$, for this reaction, using the law of mass action, is:

$$
K_{e q}=\frac{a_{C e_{C e}^{x}}^{2} a_{O_{O}^{x}} P_{O_{2}}^{\frac{1}{2}}}{a_{C e_{C e}^{\prime}}^{2} a_{V_{o}^{*}}}
$$

\footnotetext{
* This reference actually presents an oxygen non-stoichiometric model assuming regular solution theory and contains an additional excess Gibbs free energy term. Here we consider only the ideal solution portion of their model.
} 
If ideal defect theory is assumed, Equation (D-2) can be reduced to:

$$
K_{e q}=\frac{\left[C e_{C e}^{\prime}\right]^{2}\left[V_{O}^{* \bullet}\right]}{\left[C e_{C e}\right]^{2}\left[O_{O}^{x}\right]} P_{O_{2}}^{\frac{1}{2}}
$$

The brackets indicate the concentration of each chemical species per unit lattice volume. The following relationships for the concentrations of these species can be written, based on the $\mathrm{CeO}_{2}$ unit cell volume, $v_{0}:^{\dagger}$

$$
\begin{gathered}
{\left[O_{O}^{x}\right]+\left[V_{O}^{* *}\right]=\frac{8}{v_{o}}} \\
{\left[C e_{C e}^{x}\right]+\left[C e_{C e}^{\prime}\right]+\left[G d_{C e}^{\prime}\right]=\frac{4}{v_{0}}}
\end{gathered}
$$

The concentration of vacancies, $\left[V_{o}^{*}\right]$, can be expressed in terms of the deviation in stoichiometry due to the Gd doping level, and the deviation due to the $P_{\mathrm{O}_{2}}$ conditions:

$$
\left[V_{O}^{\bullet \bullet}\right]=\frac{4}{v_{0}} \delta=\frac{4}{v_{0}}\left(\delta_{0}+\delta^{\prime}\right)
$$

where $\delta=$ total oxygen non-stoichiometry $=\delta_{0}+\delta^{\prime}$

$\delta_{0}=$ deviation from stoichiometry due to the Gd doping mole fraction

$=0.20$ (for the Gd mole fraction, $x$, equal to 0.40 )

$\delta^{\prime}=$ deviation from stoichiometry due to $P_{\mathrm{O}_{2}}$

$v_{0}=$ volume of the GDC lattice unit cell

\footnotetext{
${ }^{\dagger}$ Note: An ideal $\mathrm{CeO}_{2}$ unit cell consists of a face-centered-cubic (FCC) lattice containing 4 cerium atoms and 8 oxygen atoms.
} 
The concentration of reduced Ce, $\left[\mathrm{Ce}_{C e}^{\prime}\right]$, is assumed to be only a function of $P_{\mathrm{O}_{2}}$ conditions:

$$
\left[C e_{C e}^{\prime}\right]=\frac{8}{v_{0}} \delta^{\prime}
$$

If electroneutrality is assumed at the unit cell level, the following additional relationship is valid: ${ }^{\ddagger}$

$$
\left[C e_{C e}^{\prime}\right]+\left[G d_{C e}^{\prime}\right]=2\left[V_{o}^{* \bullet}\right]
$$

With these definitions, each species concentration can be solved for and substituted into the Equation (D-3) mass action expression. $K_{e q}$ is then expressed completely in terms of the oxygen non-stoichiometry of GDC, i.e. in terms of $\delta_{0}$ and $\delta^{\prime}$ :

$$
K_{e q}=\frac{4 \delta^{\prime 2}\left(\delta_{0}+\delta^{\prime}\right) P_{O_{2}}^{1 / 2}}{\left(1-2 \delta_{0}-2 \delta^{\prime}\right)^{2}\left(2-\delta_{0}-\delta^{\prime}\right)}
$$

The standard free energy of reaction, $\Delta G^{0}$, is related to $K_{e q}$ by the expression [2]:

$$
\begin{aligned}
& \Delta G^{0}=-R T \ln K_{e q} \\
& \Delta G^{0}=-R T \ln \frac{4 \delta^{\prime 2}\left(\delta^{\prime}+\delta_{0}\right) P_{o_{2}}^{1 / 2}}{\left(1-2 \delta^{\prime}-2 \delta_{0}\right)^{2}\left(2-\delta^{\prime}-\delta_{0}\right)}
\end{aligned}
$$

\footnotetext{
${ }^{\ddagger}$ Equation (D-8) also implicitly assumes that $G d_{C e}^{\prime}$ is not reduced, and $C e_{C e}^{\prime}$ is not further reduced (i.e. to $C e_{C e}^{\prime \prime}$ ), due to $P_{\mathrm{O}_{2}}$ conditions.
} 
Equation (D-10) is the ideal defect chemistry model relating the $P_{0_{2}}$ (and $T$ ) to the oxygen non-stoichiometry of GDC. SIDE NOTE: Equation (D-10) was derived in reference [1], but the squared term in the denominator of their expression is slightly different, i.e. $\left(1-2 \delta_{0}-\delta^{\prime}\right)^{2}$, and assumed to be a typographical error. Reference [3] also derives a similar expression which matches the above result.

Equation (D-10) can now be rearranged to solve for $P_{\mathrm{O}_{2}}$ :

$$
\frac{1}{2} \ln P_{\mathrm{O}_{2}}=\ln \frac{\left(1-2 \delta^{\prime}-2 \delta_{0}\right)^{2}\left(2-\delta^{\prime}-\delta_{0}\right)}{4 \delta^{2}\left(\delta^{\prime}+\delta_{0}\right)}-\frac{\Delta G_{f i t}^{0}}{R T}
$$

Gibbs free energy ( $\Delta G^{0}$ ) data for this reaction is not typically tabulated, but limited oxygen nonstoichiometry data versus $P_{\mathrm{O}_{2}}$ can be found in the literature [4]. So the variable, $\Delta G_{f i t}^{0}$, was designated as a fitted parameter and Equation (D-11) was then used to fit the $40 \mathrm{~mol} \%$ GDC data in reference [4] using a non-linear least squares routine in MATLAB that implements the Levenberg-Marquardt algorithm [5]. The value of $\Delta G_{\text {fit }}^{0}=5.35 \times 10^{5} \mathrm{~J} / \mathrm{mol}$ obtained for this data at $1000^{\circ} \mathrm{C}$ is significantly higher than the value of $\Delta G_{\text {fit }}^{0}=2.74 \times 10^{5} \mathrm{~J} / \mathrm{mol}$ reported by Yashiro et al. for $10 \mathrm{~mol} \% \mathrm{GDC}$ at $900^{\circ} \mathrm{C}$, indicating an expected higher free energy change required for creating additional oxygen vacancies in the higher doped material. Figure D-1 displays the fit of this model over the $P_{\mathrm{O}_{2}}$ range of interest in this study. 


\section{D.2 Derivation of the GDC thermodynamic factor, A}

To derive an expression for the thermodynamic factor, Equation (D-11) is first differentiated with respect to $\delta^{\prime}$ :

$$
\frac{1}{2} \frac{\partial \ln P_{O_{2}}}{\partial \delta^{\prime}}=-\frac{2}{\delta^{\prime}}+\frac{1}{\delta_{O}+\delta^{\prime}-2}-\frac{1}{\delta_{O}+\delta^{\prime}}+\frac{4}{2 \delta_{O}+2 \delta^{\prime}-1}
$$

The chain rule is used to obtain our definition of the thermodynamic factor, $A$ :

$$
-\frac{1}{2} \frac{\partial \ln P_{O_{2}}}{\partial \delta^{\prime}}=\underbrace{-\frac{1}{2} \frac{\partial \ln P_{O_{2}}}{\partial \ln x_{v}}}_{=A} \frac{\partial \ln \frac{1}{2}\left(\delta_{O}+\delta^{\prime}\right)}{\partial \delta^{\prime}}
$$

$$
\text { and, noting that } x_{v}=\frac{\delta}{2}=\frac{\delta_{O}+\delta^{\prime}}{2}
$$

Equation (D-13) can be rearranged to give:

$$
-\frac{1}{2} \frac{\partial \ln P_{O_{2}}}{\partial \ln x_{v}}=-\frac{1}{2} \frac{\partial \ln P_{O_{2}}}{\partial \delta^{\prime}} \frac{\delta_{o}+\delta^{\prime}}{2}
$$

Finally, Equation (D-12) is substituted into (D-14), to obtain Equation (5-8) in Chapter 5:

$$
A=-\frac{1}{2} \frac{\partial \ln P_{\mathrm{O}_{2}}}{\partial \ln x_{v}}=\frac{\delta_{0}}{\delta^{\prime}}-\frac{\delta_{O}+1}{2 \delta_{0}+2 \delta^{\prime}-1}-\frac{1}{\delta_{0}+\delta^{\prime}-2}
$$


In reference [6], the Yashiro et al. derived point defect theory model was used to derive the thermodynamic factor, resulting in the denominator of the second term in Equation (D-15) having a slight error, i.e. $\delta_{0}+2 \delta^{\prime}-1$. This difference resulted in an error of less than $3 \%$ in the calculation of $A$.

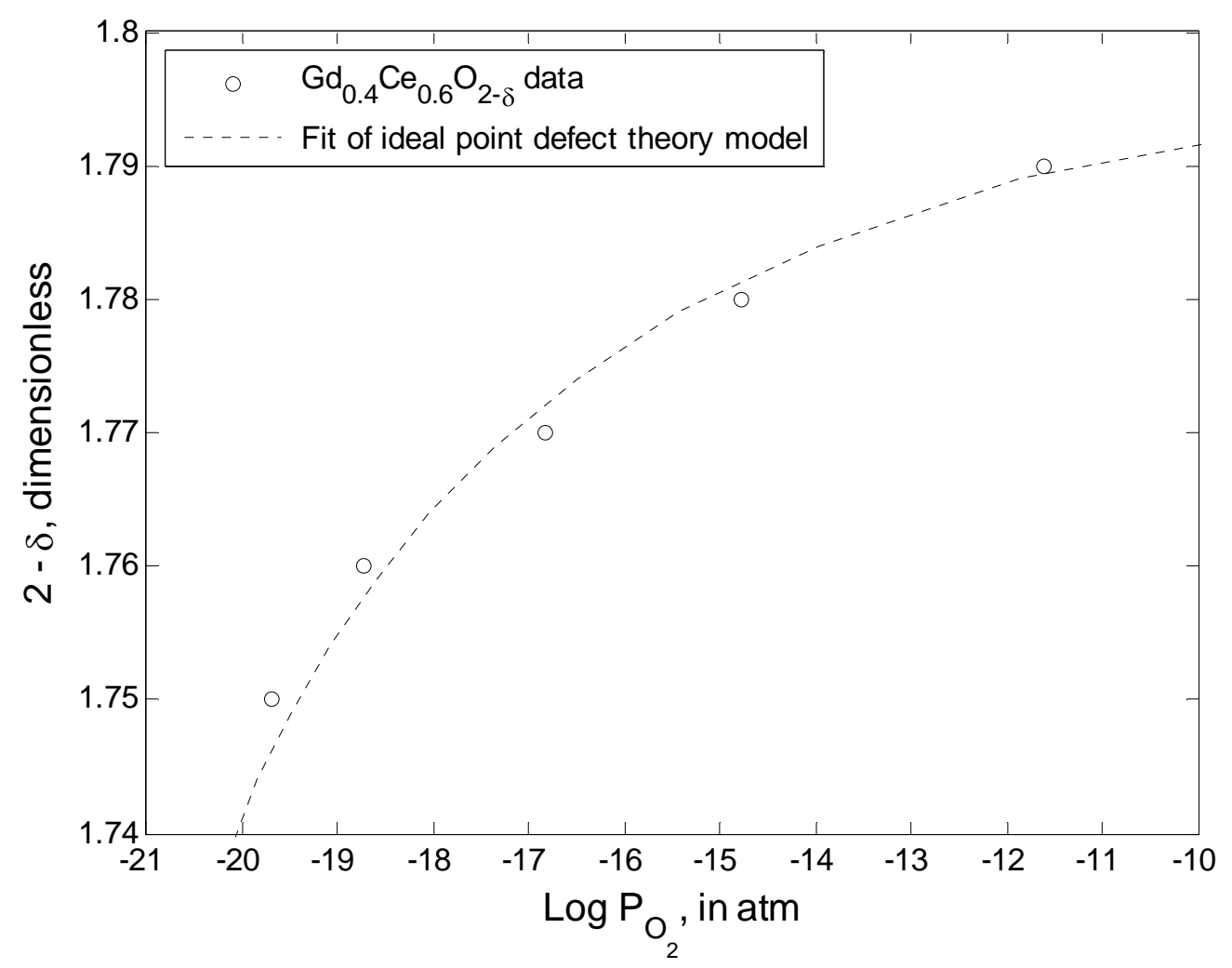

Figure D-1. Ideal point theory defect model fit to 40 mol\% GDC data. GDC oxygen non-stoichiometry data is from reference [4]. Equation (D-11) provides the model fit.

\section{References:}

[1] K Yashiro, S Onuma, A Kaimai, Y Nigara, T Kawada, J Mizusaki, et al. Mass transport properties of $\mathrm{Ce}_{0.9} \mathrm{Gd}_{0.1} \mathrm{O}_{2 \text {-delta }}$ at the surface and in the bulk, Solid State Ionics. 152-153 (2002) 469-476. 
[2] PW Atkins, Physical Chemistry, first ed., W. H. Freeman and Company, San Francisco, CA, 1978.

[3] D Perez-Coll, D Marrero-Lopez, JC Ruiz-Morales, P Nunez, Abrantes, J. C. C., JR Frade. Reducibility of $\mathrm{Ce}_{1-\mathrm{x}} \mathrm{Gd}_{\mathrm{x}} \mathrm{O}_{2 \text {-delta }}$ in prospective working conditions, J. Power Sources. 173 (2007) 291-297.

[4] B Zachau-Christianson, T Jacobsen, K West, S Skaarup, Electrochemical determination of the oxygen stoichiometry of doped ceria, in: Singhal SC, Iwahara $\mathrm{H}$ (Eds.), Proceedings of the Third International Symposium on Solid Oxide Fuel Cells, SOFC III, PV93-4, Pennington, NJ ed., The Electrochemical Society, Pennington, NJ, 1993, pp. 104-111.

[5] A Constantinides, N Mostoufi, Numerical Methods for Chemical Engineers with MATLAB Applications, first ed., Prentice Hall PTR, Upper Saddle River, NJ, 1999.

[6] RD Green, CC Liu, SB Adler. Carbon dioxide reduction on gadolinia-doped ceria cathodes, Solid State Ionics. 179 (2008) 647-660. 


\section{Appendix E}

\section{DERIVATION OF THE CO-CO 2 EXCHANGE MODEL FOR A NON-ZERO VACANCY FLUX AT THE CURRENT COLLECTOR \\ SURFACE}

\section{E.1 Introduction}

In Chapter 4, the $\mathrm{CO}-\mathrm{CO}_{2}$ exchange model was derived for the following boundary conditions:

$$
\begin{gathered}
\text { @ } \mathrm{y}=0: N_{v}=-c_{0}(1-\varepsilon) D_{v}^{e f f} A \frac{\partial x_{v}}{\partial y}=\frac{-i}{2 F} \\
\text { @ } y=L: N_{v}=-c_{0}(1-\varepsilon) D_{v}^{e f f} A \frac{\partial x_{v}}{\partial y}=(1-\varepsilon) r
\end{gathered}
$$

But, the boundary condition at the current collector surface, described by Equation (E-2), was set equal to zero, or:

$$
\frac{\partial x_{v}}{\partial y}=0 \text { i.e. } r=0 @ y=L
$$

The boundary condition in Equation (E-2) is an approximation based on assumption \#5 made in Chapter 4, and is based on the rationale that the $\mathrm{CO}-\mathrm{CO}_{2}$ exchange reaction rate, $r$, is high near the electrode/electrolyte interface $(y=0)$ and decreases through the thickness of the electrode as one approaches the current collector interface at $y=L$. In a porous electrode consisting of a mixed ionic-electronic conductor (MIEC) like GDC, the charge (or current) carrier must be primarily an ionic flux near the 
electrode/electrolyte interface, and primarily an electronic flux at the current collector interface [1], but this does not require the reaction rate be negligible for these boundary conditions to hold. For the $\mathrm{CO}-\mathrm{CO}_{2}$ exchange model, a measure of how much of the thickness of the electrode is 'active' for the $\mathrm{CO}-\mathrm{CO}_{2}$ exchange reaction is the active electrode length, or $\phi=\frac{L}{l_{\delta}}$, where $l_{\delta}$ is the utilization length of the electrode. But for the impedance data fitted to the $\mathrm{CO}-\mathrm{CO}_{2}$ exchange model in Chapter 5, the value of the fitted parameter, $\phi$, is less than 1.0 for some cases, implying the utilization length exceeds the thickness of the electrode. This implies the $\mathrm{CO}-\mathrm{CO}_{2}$ exchange reaction rate is non-zero at $y=L$, and challenges our zero flux boundary condition assumption. The objective of this appendix is to address this apparent disagreement with the boundary condition defined in Equation (E-2).

\section{E.2 Rationale for zero vacancy flux at $y=L$}

At the current collector surface, the oxygen vacancy generation rate is:

$$
\text { Vacancy generation rate }\left.\right|_{y=L}=\left.(1-\varepsilon) \mathrm{A}_{e l} r\right|_{y=L}
$$

where $\mathrm{A}_{e l}=$ the electrode imprinted area on the electrolyte

Within the entire electrode, the oxygen vacancy generation rate can be expressed as:

$$
\text { Total vacancy generation rate in electrode }=a \mathrm{~A}_{e l} \int_{0}^{L} r d y
$$

The rationale behind this assumption is as follows: The definition of the $\phi$ parameter in the $\mathrm{CO}-\mathrm{CO}_{2}$ exchange model indicates the outer electrode surface becomes 
kinetically active for $\phi<1.0$. If we examine the extreme case where $\phi \rightarrow 0$, the entire electrode is kinetically active and the reaction rate per unit surface area, $r$, is approximately constant over the entire electrode thickness, including the outer surface. For this condition, we can compare the reaction rates of the outer surface versus the entire electrode by calculating the ratio of the outer electrode surface area to the total internal surface area of the electrode, i.e.:

$$
\frac{\text { Area of electrode outer surface }}{\text { Total area of internal electrode surface }}=\frac{(1-\varepsilon) \mathrm{A}_{\mathrm{el}}}{a \mathrm{~A}_{\mathrm{el}} L}=\frac{(1-\varepsilon)}{a L}
$$

For the estimated microstructure properties of the GDC electrode, $\varepsilon=0.5$, $a=10,000 \mathrm{~cm}^{-1}$, and $L=40 \times 10^{-4} \mathrm{~cm}$, this ratio translates to a value of 0.0065 , indicating that even in this most extreme case, less than $1 \%$ of the reaction occurs at the outer surface. Of course, for electrodes that are much thinner (e.g. fabricated via sputtering or other thin film techniques), have low porosity, or lower $a$ values, this assumption would have to be revisited.

\section{E.3 Solution of $\mathrm{CO}-\mathrm{CO}_{2}$ exchange model for non-zero flux at $y=L$}

In the previous section, a qualitative rationale was given for our assumption of a zero vacancy flux at the current collector interface. But a closed-form solution does exist exists for the vacancy flux set equal to the reaction rate at the outer surface of the electrode, as given in Equation (E-2). In this section, a derivation of this alternate 
solution is provided along with a comparison of this solution with the Chapter 4 model solution, on a single set of impedance data for $\mathrm{CO}-\mathrm{CO}_{2}$ exchange on a GDC electrode.

The derivation follows the same procedure described in Chapter 4, starting with Equation (4-15), the equation of continuity for oxygen vacancies, in non-dimensional form and transformed to frequency space:

$$
\frac{\partial^{2} \tilde{\psi}}{\partial \xi^{2}}=(1+j \sigma) \tilde{\psi}
$$

The two boundary conditions in Equations (E-1) and (E-2) are converted to nondimensional form:

$$
\begin{aligned}
& \text { @ } \xi=0: \frac{\partial \tilde{\psi}}{\partial \xi}=\alpha \\
& @ \xi=\phi: \frac{\partial \tilde{\psi}}{\partial \xi}=\beta \tilde{\psi}
\end{aligned}
$$

where $\beta=\frac{c_{O} D_{v}^{\text {eff }} X_{v}^{\text {eqb }}}{l_{\delta} \Re_{0}}$ is an additional fitted parameter, and $\mathrm{R}_{\text {chem }}, \mathrm{t}_{\text {chem }}$, and $\phi$ are the same as defined in Table 4-1.

The general solution to the second order homogeneous differential equation in Equation (E-7) was given as Equation (4-20) in Chapter 4:

$$
\tilde{\psi}=c_{1} \cosh (\sqrt{1+j \sigma} \xi)+c_{2} \sinh (\sqrt{1+j \sigma} \xi)
$$

where $c_{1}, c_{2}$ are integration constants 
Applying the boundary conditions to solve for the integration constants, we obtain:

$$
\tilde{\psi}=-\frac{\alpha \beta \cosh [\sqrt{1+j \sigma}(\phi-\xi)]+(\sqrt{1+j \sigma})^{-1} \sinh [\sqrt{1+j \sigma}(\phi-\xi)]}{\cosh [\phi \sqrt{1+j \sigma}]+\beta \sqrt{1+j \sigma} \sinh [\phi \sqrt{1+j \sigma}]}
$$

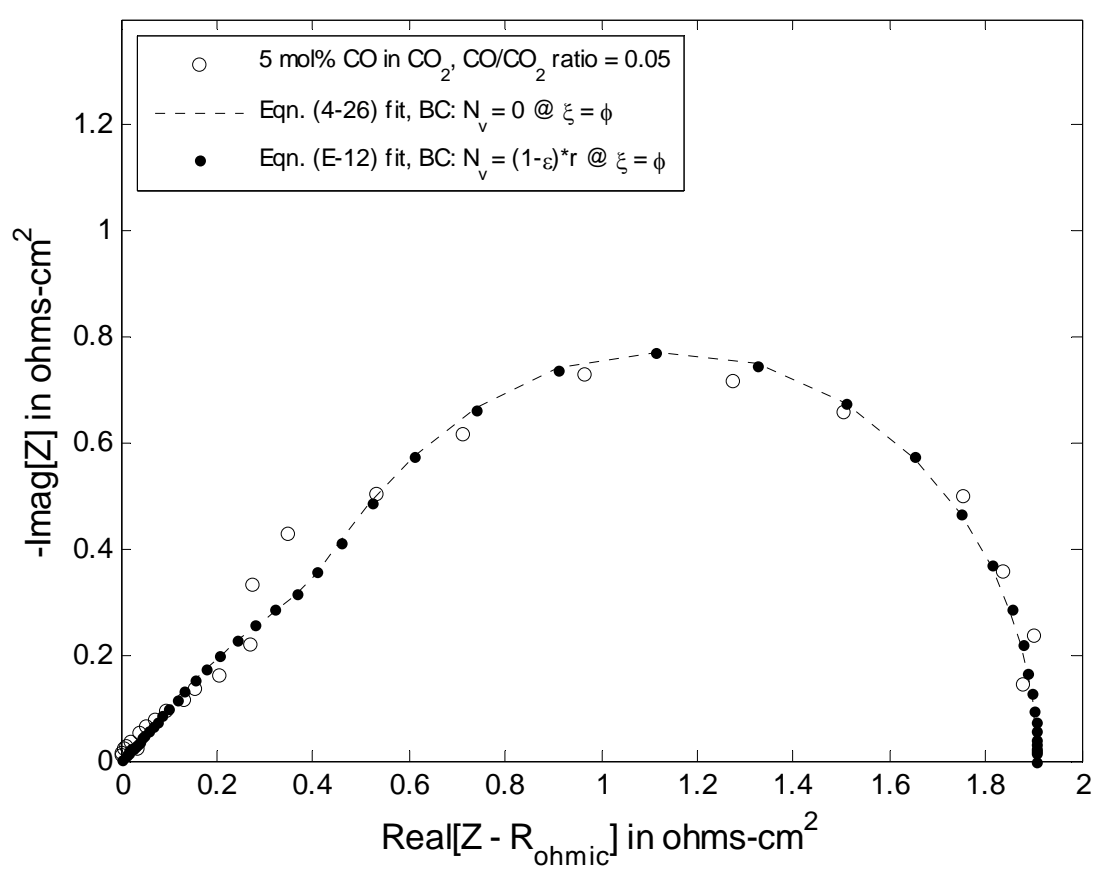

Figure E-1. Comparison of CO- $\mathrm{CO}_{2}$ model fits for a zero flux and a non-zero vacancy flux boundary condition at the current collector interface.

The solution is a bit more complicated than for $Z_{\text {chem }}$ given in Equation (4-26):

$$
Z_{\text {chem }}=R_{\text {chem }} \frac{\beta \cosh \left(\phi \sqrt{1+j \omega t_{\text {chem }}}\right)+\frac{1}{\sqrt{1+j \omega t_{\text {chem }}}} \sinh \left(\phi \sqrt{1+j \omega t_{\text {chem }}}\right)}{\cosh \left(\phi \sqrt{1+j \omega t_{\text {chem }}}\right)+\beta \sqrt{1+j \omega t_{\text {chem }}} \sinh \left(\phi \sqrt{1+j \omega t_{\text {chem }}}\right)}
$$




$$
\begin{aligned}
& \text { where } \beta=\frac{c_{O} D_{v}^{\text {eff }} x_{v}^{\text {eqb }}}{l_{\delta} \Re_{0}} \text { is an additional fitted parameter, and } \\
& \mathrm{R}_{\text {chem }}, \mathrm{t}_{\text {chem }} \text {, and } \phi \text { are defined in Table } 4-1 .
\end{aligned}
$$

As an example, we fitted the impedance spectra on a GDC electrode for T = $900^{\circ} \mathrm{C}$ and $\mathrm{CO} / \mathrm{CO}_{2}$ ratio of 0.05 to Equation (E-12) above. Figure E-1 shows this comparison between the two fits.

A comparison of the fitted parameters with those fitted to Equation (4-24) in the paper is given in Table E-1:

\begin{tabular}{|l|l|l|l|l|c|}
\hline Equation No. & Boundary Condition & $R_{\text {chem }}$ & $t_{\text {chem }}$ & $\phi$ & $\beta$ \\
\hline$(4-26)$ & $N_{v}=0 @ \xi=\phi$ & 1.38 & 1.38 & 0.91 & N/A \\
\hline (E-12) & $N_{v}=(1-\varepsilon) r @ \xi=\phi$ & 1.46 & 1.56 & 0.90 & 9.12 \\
\hline
\end{tabular}

Table E-1. Comparison of fitted parameters for the $\mathrm{CO}-\mathrm{CO}_{2}$ model solved for zero and non-zero vacancy flux boundary conditions at the current collector interface.

The comparison is good, given the sensitivity of some of the parameters to both these non-linear fits. We chose to stick with Equation (4-26) in Chapter 4 because of its simpler form and one less fitting parameter.

\section{Reference:}

[1] J Newman, KE Thomas-Alyea, Electrochemical Systems, third ed., WileyInterscience, Hoboken, NJ, 2004. 


\section{Appendix F}

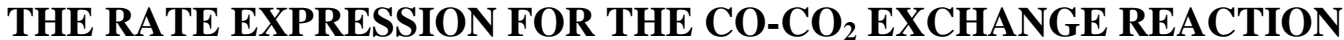

In this appendix, a brief discussion of the form of the rate expression in Equation (4-8) in Chapter 4 is provided. The intent is to provide the reader with a rationale for the form of this expression, as opposed to a formal derivation of a non-linear rate expression.

It is assumed that the $\mathrm{CO}-\mathrm{CO}_{2}$ exchange proceeds by a multi-step reaction mechanism. Below is a possible 4-step reaction mechanism sequence:

$$
\begin{gathered}
\text { adsorption: } \mathrm{CO}_{2}+\mathrm{V}_{\mathrm{O}_{s}}^{\bullet \bullet}+2 e^{-} \rightleftharpoons\left(\mathrm{CO}_{2}\right)_{\mathrm{O}_{s}}^{x} \\
\text { dissociation: }\left(\mathrm{CO}_{2}\right)_{O_{s}}^{x} \rightleftharpoons \mathrm{CO}+\mathrm{O}_{\mathrm{O}_{s}}^{x} \\
\text { incorporation: } \mathrm{O}_{\mathrm{O}_{s}}^{x}+V_{O_{b}}^{\bullet \bullet}+2 e^{-} \rightleftharpoons \mathrm{O}_{O_{b}}^{x}+V_{O_{s}}^{\bullet \bullet} \\
\text { overall reaction: } \mathrm{CO}_{2}+V_{O}^{\bullet \bullet}+2 e^{-} \rightleftharpoons \mathrm{CO}+\mathrm{O}_{O}^{x}
\end{gathered}
$$

From heterogeneous catalysis studies of $\mathrm{CO}$ oxidation on ceria [1-3], it is believed that $\mathrm{CO}_{2}$ adsorption/desorption, Equation (F-1), is most likely a rate-determining step.

The $\mathrm{CO}-\mathrm{CO}_{2}$ exchange reaction on GDC is assumed to have a single rate-determining reaction step. For an individual reaction step in a reaction mechanism, Adler et al. [4] 
derive the following general form kinetic rate expression utilizing transition state theory $[5]:$

$$
r=k e^{\frac{-\Delta G_{t}^{0}}{R T}} e^{\frac{-(1-\beta) \Delta E}{R T}} \prod_{i} c_{i}\left(1-e^{\frac{-\Lambda}{R T}}\right)
$$

where $k=$ a rate factor, independant of composition, and analogous to an Arrhenius pre-exponential factor

$\Delta G_{f}^{0}=$ is the unperturbed free energy for this reaction step in the forward direction

$\Delta E=$ a shift in the free energy of reaction associated with the finite driving force, $\Lambda$

$\beta=$ a reaction symmetry parameter analogous to that used in electrochemical kinetics

$\Lambda=$ total free energy driving force for this reaction step

$c_{i}=$ the concentrations of the reacting species in the forward direction

For the overall $\mathrm{CO}-\mathrm{CO}_{2}$ exchange reaction at equilibrium:

$$
0=R T \ln \frac{P_{\mathrm{CO}_{2}} X_{v}^{\frac{1}{2}}}{P_{\mathrm{CO}}}-\Delta G_{r \times n}^{0}+2 E_{F}
$$

where $\Delta G_{r \times n}^{0}=$ the standard free energy of reaction

$E_{F}=$ the Fermi energy measured relative to the value for GDC at full oxygen stoichiometry

The driving force, $\Lambda$, is defined as a displacement from this equilibrium condition of the exchange reaction, or:

$$
\begin{aligned}
\Lambda & =R T \ln \left(P_{O_{2}}^{\text {gas }}\right)^{\frac{1}{2}} X_{v}^{\frac{1}{2}}-\Delta G_{r x n}^{0}+2 E_{F} \\
& =\frac{R T}{2} \ln \frac{P_{O_{2}}^{\text {gas }}}{f_{O_{2}}^{\text {solid }}}
\end{aligned}
$$


For convenience in solving Equation (4-10) in Chapter 4, $P_{\mathrm{CO}}$ and $P_{\mathrm{CO}_{2}}$ are expressed in terms as the $P_{\mathrm{O}_{2}}^{\text {gas }}$ in equilibrium with the $\mathrm{CO}$ and $\mathrm{CO}_{2}$ in the gas phase, and the bulk mole vacancy fraction is expressed in terms of the oxygen fugacity in the bulk GDC solid, $f_{\mathrm{O}_{2}}^{\text {solid }}$, in equilibrium with the solid at a composition, $x_{v}$. This overall driving force would be distributed over the entire set of reaction steps in a reaction, but for a mechanism with a rate-determining step, the other reaction steps are assumed to be quasiequilibrated, and their associated driving forces approach zero.

Equation (F-5) is of the form of a non-equilibrium thermodynamic rate expression, where $\Lambda$ can be referred to as a thermodynamic driving force or "affinity" [6]. The prefixed term in Equation (F-5) is defined in Equation 4-7 as $\mathfrak{R}_{0}$, the surface exchange rate; this is equivalent to the exchange rate (or $r=r_{f}=r_{b}$ where $r_{f}$ and $r_{b}$ are forward and backward reaction rates) for a chemical reaction in the limit of equilibrium.

\section{References:}

[1] M Breysse, M Guenin, B Claudel, H Latreille, J Veron. Catalysis of carbon monoxide by cerium dioxide I. Correlations between catalytic activity and electrical conductivity, J. Catalysis. 27 (1972) 275-280.

[2] M Breysse, M Guenin, B Claudel, J Veron. Catalysis of carbon monoxide by cerium dioxide II. Microcalorimetric investigation of adsorption and catalysis, J. Catalysis. 28 (1973) 54-62. 
[3] M Primet, E Garbowski, Fundamentals and applications of ceria in combustion reactions, in: Trovarelli A (Ed.), Catalysis by Ceria and Related Materials, vol. 2, Imperial College Press, London, 2002, pp. 407-429.

[4] SB Adler, XY Chen, JR Wilson. Mechanisms and rate laws for oxygen exchange on mixed-conducting oxide surfaces, J. Catalysis. 245 (2007) 91-109.

[5] M Boudart, Kinetics of Chemical Processes, Prentice-Hall, Englewood Cliffs, NJ, 1968.

[6] De Groot, S. R., P Mazur, Non-Equilibrium Thermodynamics, first ed., NorthHolland, Amsterdam, the Netherlands, 1962. 


\section{Appendix $G$}

\section{A BRIEF INTRODUCTION TO KRÖGER-VINK DEFECT NOTATION}

A commonly used notation in the literature for denoting crystal defect chemistry is Kröger-Vink defect notation [1] ${ }^{*}$. In this appendix, a brief introduction to KrögerVink notation is provided to aid the reader in interpreting a number of defect chemistry expressions in the main text of this document.

In Kroger-Vink notation, the main letter denotes the atom of interest, a subscript denotes the lattice position, and a superscript denotes the charge of the atom relative to the neutral (or ideal) lattice position. Examples are given below:

$\begin{array}{ll}\text { Symbol } & \text { Description } \\ \mathrm{Ce} & \text { Cerium atom } \\ \mathrm{C} e_{C e}^{x} & \text { Cerium atom on cerium lattice site } \\ G d_{C e}^{\prime} & \text { Gadolinium atom on cerium lattice site } \\ V & \text { vacancy } \\ V_{O}^{\bullet \bullet} & \text { vacancy on oxygen lattice site } \\ e^{\prime} & \text { free electron } \\ h^{\bullet} & \text { free hole } \\ C e_{i}^{\bullet . \bullet} & \text { Ce atom at an interstitial site } \\ O_{i}^{\prime \prime} & \text { O atom at an interstitial site }\end{array}$

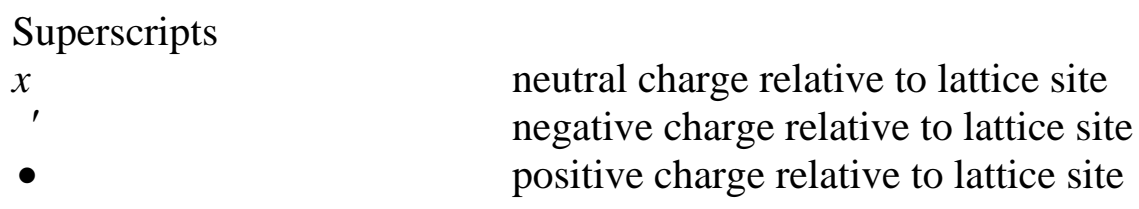

* See reference [2] for details and rationale for the choice of this notation. 


\section{References:}

[1] FA Kroger, HJ Vink, Relations between the concentrations of imperfections in crystalline solids, in: Seitz F, Turnull D (Eds.), Solid State Physics - Advances in Research and Applications, Academic Press, New York, NY, 1956, pp. 431-435.

[2] P Knauth, HL Tuller. Solid-State Ionics: roots, status and future prospects, J. Am. Ceram. Soc. 85 (2002) 1654-1680. 


\section{Appendix $\mathrm{H}$}

\section{SAMPLE MATLAB M-FILE LISTING FOR PELLET GEOMETRY ANALYSES}

$\%$ This is an M-file generated using the COMSOL Multiphysics software $\%$ for the baseline pellet electrolyte geometry. MATLAB script was $\%$ added to perform an impedance simulation by varying the frequency, $\%$ along with additional commands extracting current density at $\%$ various boundary conditions and generating various plots.

\% COMSOL Multiphysics Model M-file

$\%$ Generated by COMSOL 3.3 (COMSOL 3.3.0.405, \$Date: 2006/08/31

$\% 18: 03: 47 \$$ )

\% Some geometry objects are stored in a separate file.

$\%$ The name of this file is given by the variable 'flbinaryfile'.

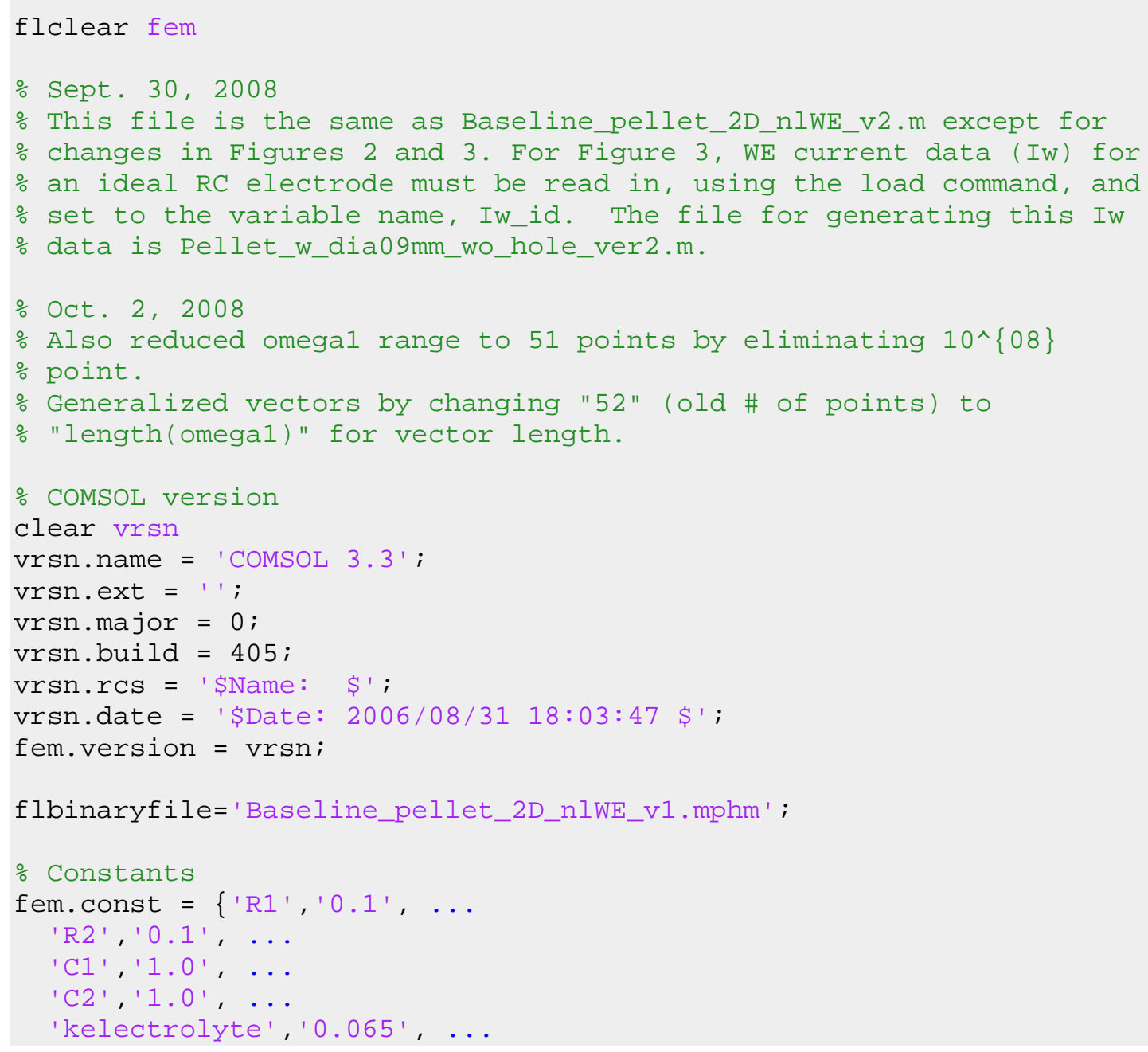




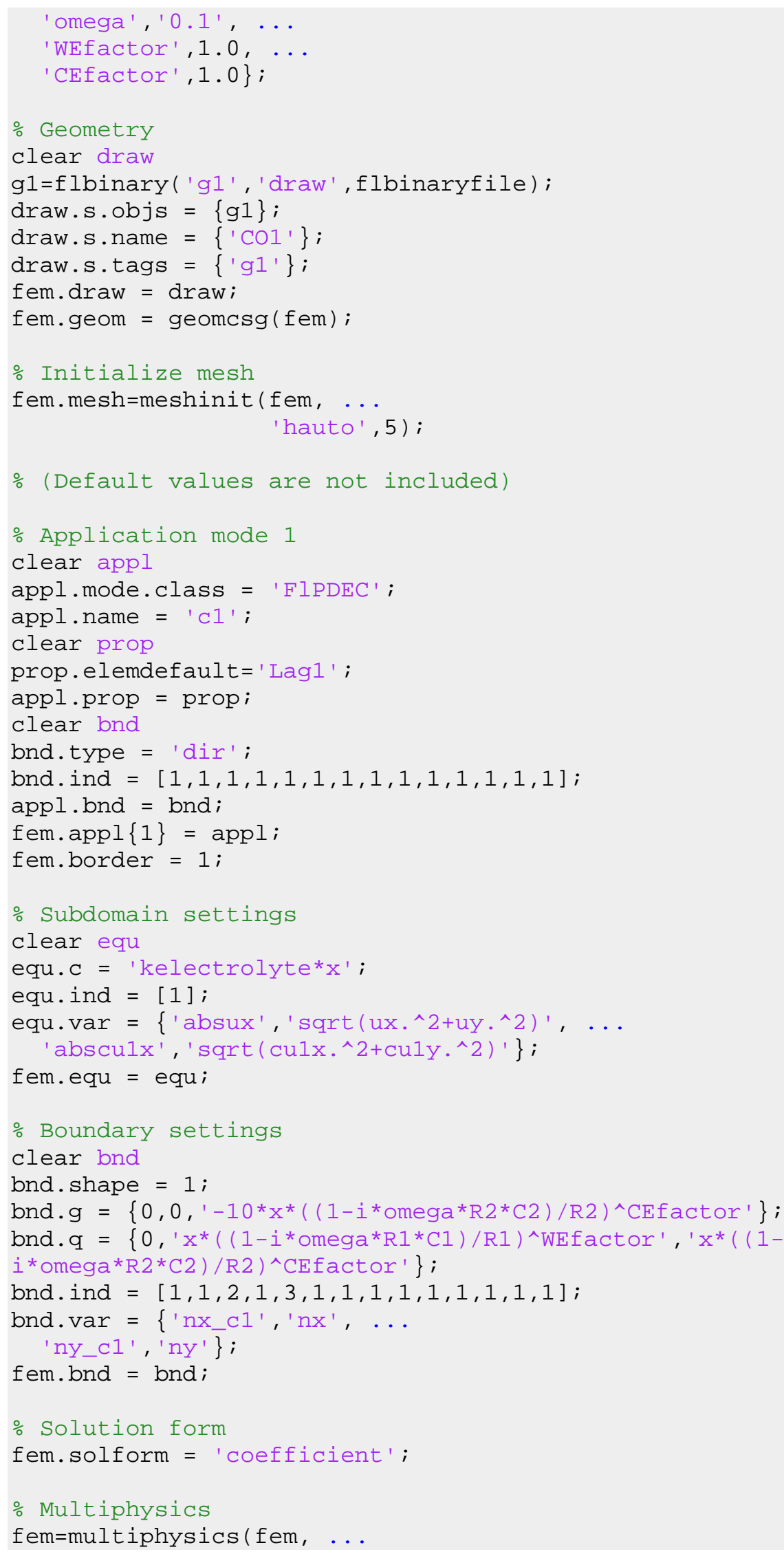




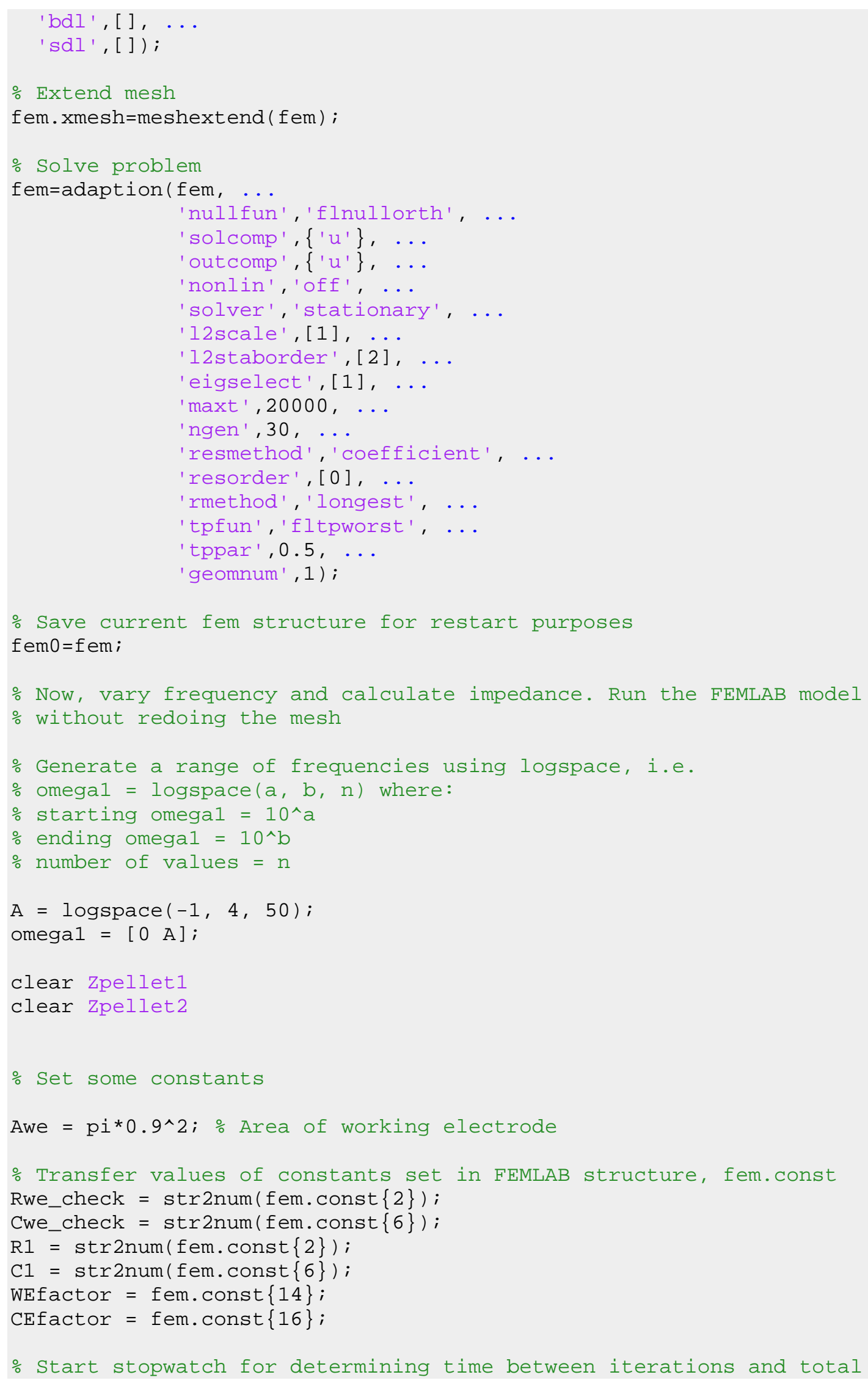




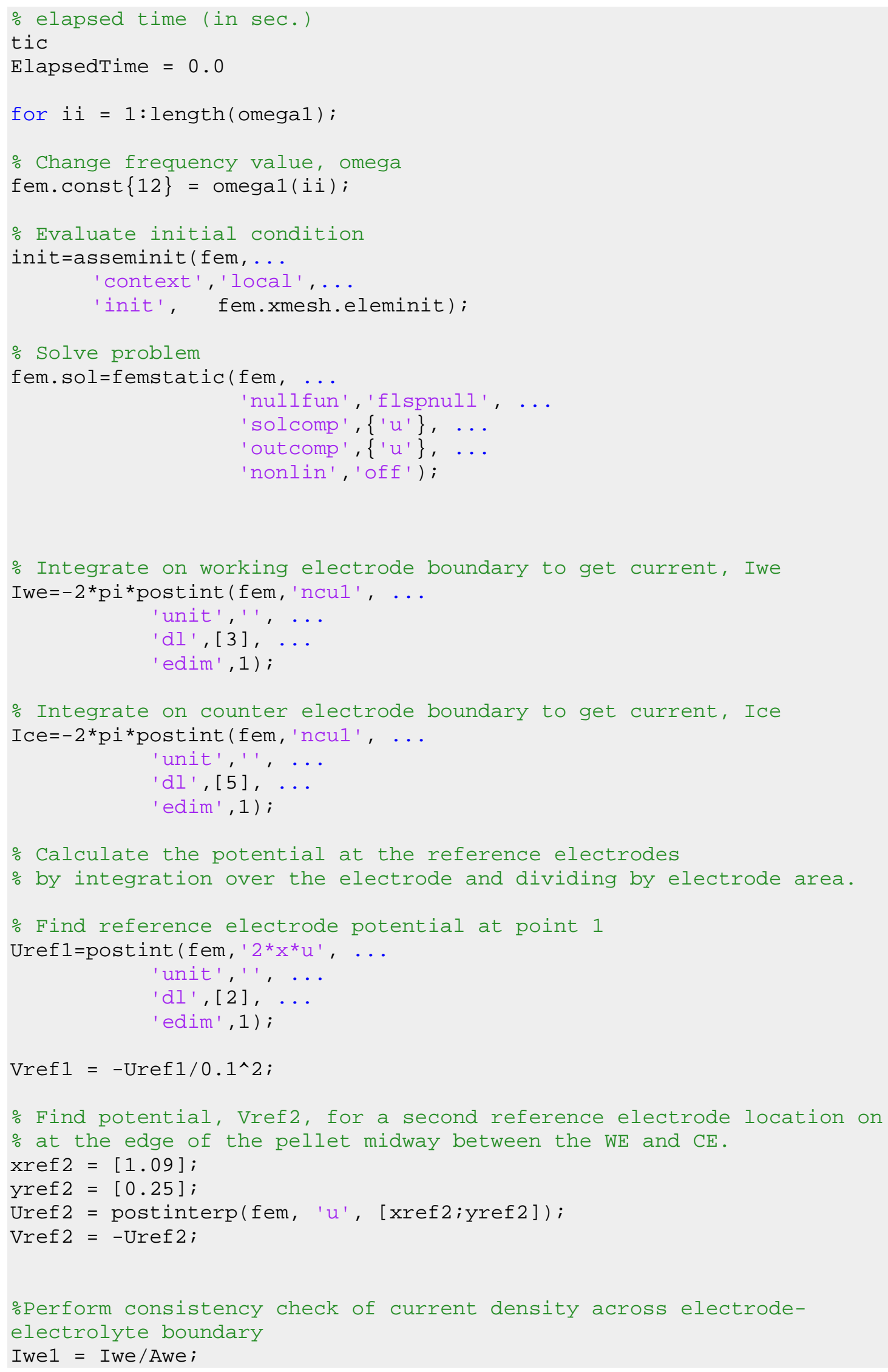

\% Integrate on working electrode boundary to get current, Iwe Iwe $=-2{ }^{*}$ pi ${ }^{*}$ postint (fem, 'ncu1',$\ldots$ 


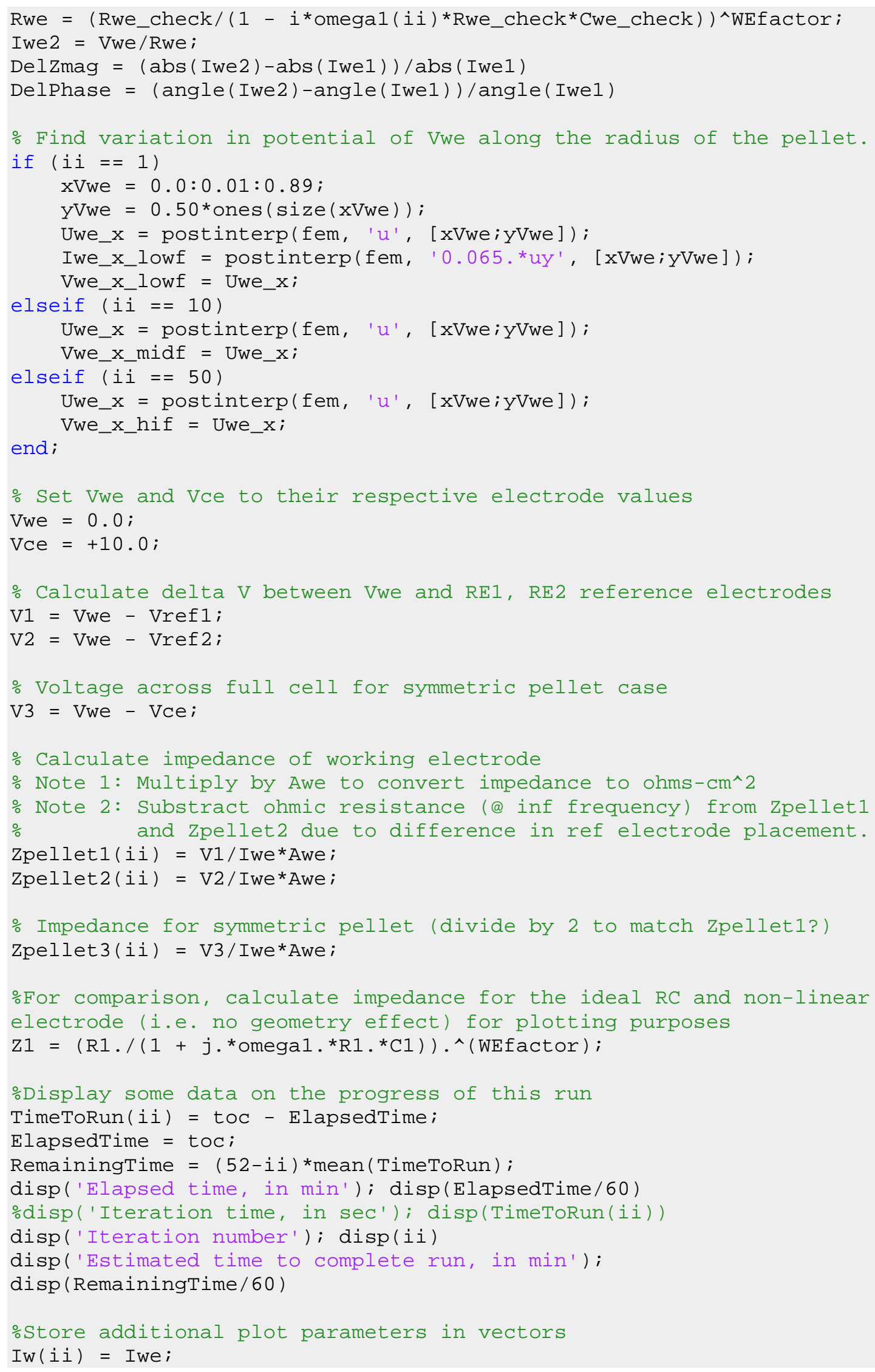


Ic $(i i)=$ Ice;

$V w(i i)=V w e ;$

$\operatorname{Vr} 1(i i)=\operatorname{Vref1}$

$\operatorname{Vr} 2(i i)=\operatorname{Vref2}$;

end

\%Calculate current density as a function of pellet radius at $\%$ omega $=0$

Iwe_x_hole $=$-Iwe_x_lowf./Iw(1). *Awe;

\% Subtract ohmic resistance from electrode impedance

Zp1 = (Zpellet1 $-\operatorname{real}($ Zpellet1 $($ length $($ omega1 $)))) ;$

$Z p 2=($ Zpellet2-real $($ Zpellet2 $($ length $($ omega1 $)))) ;$

Zp3 $=($ Zpellet3 $-\operatorname{real}($ Zpellet3 $($ length $($ omega1 $)))) / 2$;

\% Calculate error compared to ideal electrode, Z1

Z1_real = Z1(1) - Z1 (length $($ omega 1$))$;

Zp1_abs_error $=(Z p 1(1)-Z 1(1)) / Z 1$ real

Zp1_phase_error $=\max (\operatorname{angle}(Z \mathrm{p} 1-\mathrm{Z1}) / \mathrm{pi})$

Zp2_abs_error $=\max \left(\operatorname{abs}\left((Z p 2(1)-Z 1(1)) / Z 1 \_r e a l\right)\right)$

Zp2_phase_error $=\max (\operatorname{angle}(Z \mathrm{p} 2-\mathrm{Z1}) / \mathrm{pi})$

Zp3_abs_error $=\max \left(\operatorname{abs}\left((Z p 3(1)-Z 1(1)) / Z 1 \_r e a l\right)\right)$

Zp3_phase_error $=\max (\operatorname{angle}(Z \mathrm{p} 3-\mathrm{Z1}) / \mathrm{pi})$

\% BEEP to announce analysis run is complete

beep on;

beep

beep

beep off;

\% Plot results as a Nyquist (or Cole-Cole) plot

hold on

figure(1);

plot (real(Zp1), -imag(-Zp1), 'b:o', ...

real $(Z p 2),-\operatorname{imag}(-Z p 2), \quad ' r: .1, \ldots$

real(Zp3), - imag(-Zp3), 'k:s', ...

real(Z1), - $\operatorname{imag}(Z 1), \quad r-1, \ldots$

'MarkerSize', 5)

box on

$\operatorname{axis}\left(\left[\begin{array}{lllll}0 . \odot & 0.15 & 0 . \odot & 0.10\end{array}\right)\right.$

xlabel('Real[Z - R_\{\Omega\}]')

ylabel( ' - Imag [Z] ')

\%title('Z for a hole pellet with different RE locations')

legend('RE1 (in center location)', 'RE2 (in edge location)',...

'Full cell','Ideal RC electrode')

hold off

deltaV_RE1_Re $=(\operatorname{real}(\mathrm{Vr} 1)-$

real(Vr1(length (omega1)) ))/real $(\operatorname{Vr} 1($ length(omega1)) );

deltaV_RE1_Im $=($ imag $(\operatorname{Vr} 1)$ -

$\operatorname{imag}(\operatorname{Vr} 1($ length (omega1)) ) )/real $(\operatorname{Vr} 1($ length (omega1)) );

deltaV_RE2_Re $=($ real $($ Vr2 $)$ -

real $(\operatorname{Vr} 2($ length (omega1) $))) / \operatorname{real}(\operatorname{Vr} 2($ length (omega1) $))$; 


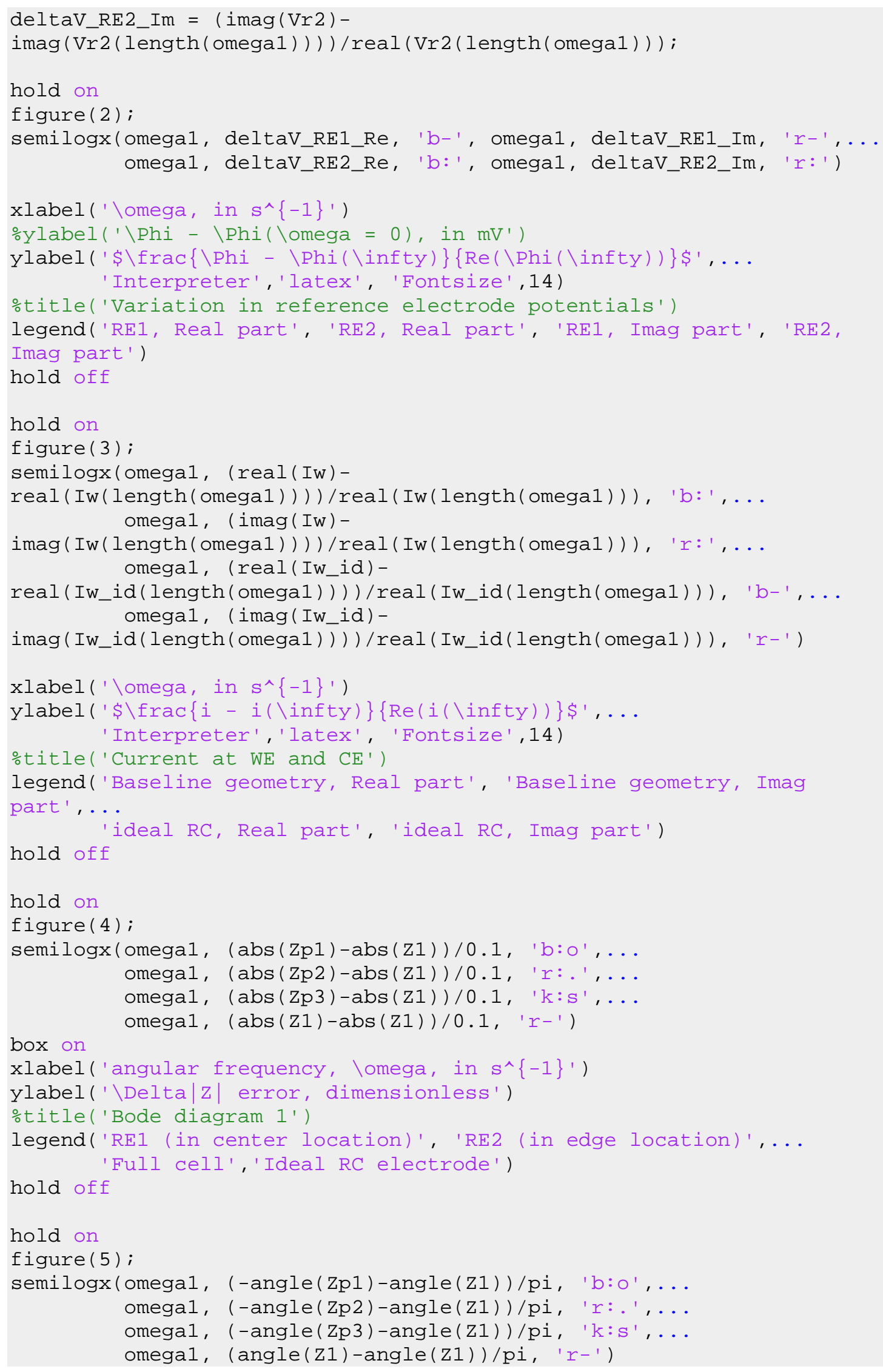




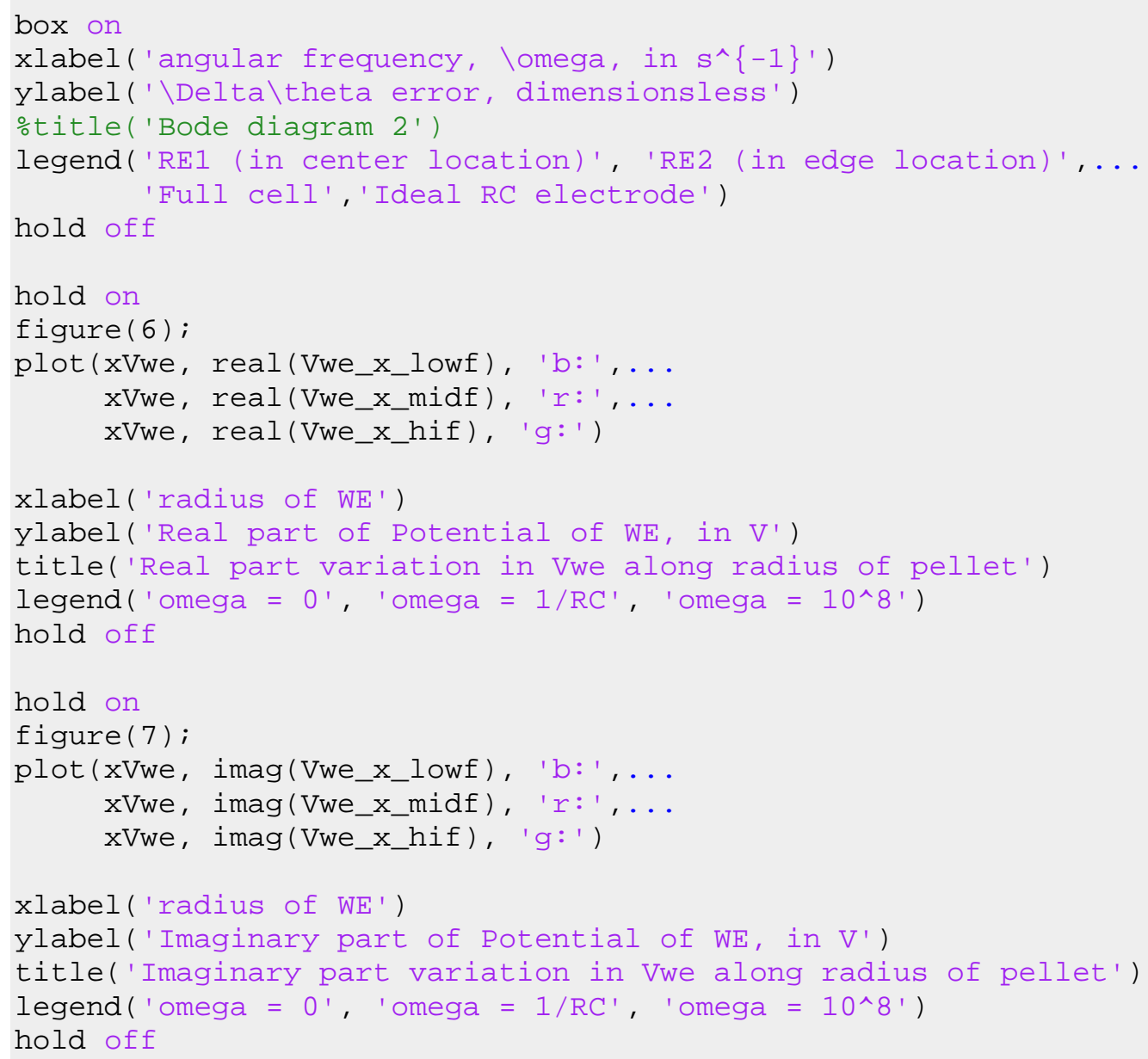




\section{Appendix I}

\section{SAMPLE MATLAB M-FILE LISTING FOR FITTING IMPEDANCE DATA

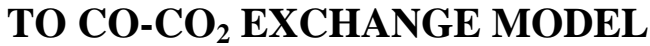

\footnotetext{
$\%$ This is an example MATLAB M-file written written to perform data

$\%$ data reduction of the GDC impedance data. The MATLAB script loads

$\%$ various sets of impedance data directly from the data files

$\%$ generated by ZPlot (Scribner Assoc.) impedance instrumentation

$\%$ software, fits the data to the $\mathrm{CO}-\mathrm{CO} 2$ exchange porous electrode

$\%$ model using the non-linear LM algorithm, and generates various

$\%$ plots.

$\%$ This particular sample $M$ file that plots $Z$ for the GDCO5 cell in $\% 5 \%, 20 \%$, and $33 \%$ CO in $\mathrm{CO} 2$ at a temperature of $900 \mathrm{deg}$. C.
}

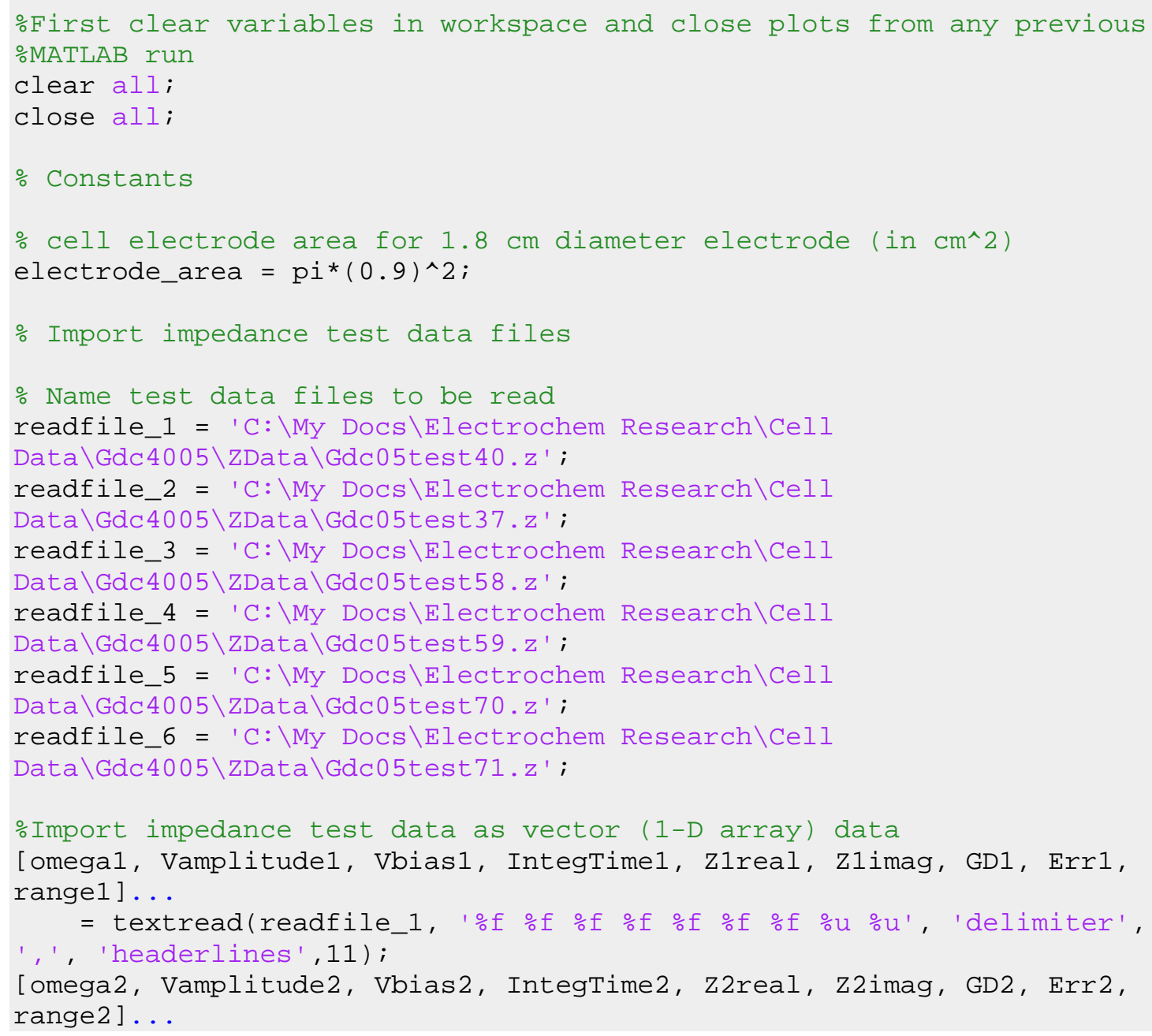


= textread(readfile_2, '\%f \%f \%f \%f \%f \%f \%f \%u \%u', 'delimiter', ', ', 'headerlines',11);

[omega3, Vamplitude3, Vbias3, IntegTime3, Z3real, Z3imag, GD3, Err3, range3]...

= textread(readfile_3, '\%f \%f \%f \%f \%f \%f \%f \%u \%u', 'delimiter', ', ', 'headerlines', 11);

[omega4, Vamplitude4, Vbias4, IntegTime4, Z4real, Z4imag, GD4, Err4, range4]...

= textread(readfile_4, '\%f \%f \%f \%f \%f \%f \%f $\% u$ \%u', 'delimiter', ', ', 'headerlines',11);

[omega5, Vamplitude5, Vbias5, IntegTime5, Z5real, Z5imag, GD5, Err5, range5]...

= textread(readfile_5, '\%f \%f \%f \%f \%f \%f \%f \%u \%u', 'delimiter', ', ', 'headerlines',11);

[omega6, Vamplitude6, Vbias6, IntegTime6, Z6real, Z6imag, GD6, Err6, range6]...

= textread(readfile_6, '\%f \%f \%f \%f \%f \%f \%f $\% u$ \%u', 'delimiter', ', ', 'headerlines', 11);

\%Remove overlapping data pts in low frequency sweeps

omega2 $(1: 1: 11)=[]$;

Z2real $(1: 1: 11)=[] ;$

$\operatorname{Z2imag}(1: 1: 11)=[] ;$

$\operatorname{omega} 4(1: 1: 11)=[] ;$

Z4real $(1: 1: 11)=[]$;

$\operatorname{Z4imag}(1: 1: 11)=[]$;

$\operatorname{omega6}(1: 1: 11)=[] ;$

Z6real $(1: 1: 11)=[]$;

$\operatorname{Z6imag}(1: 1: 11)=[] ;$

\% Concatenate $Z$ and omega value arrays for separate high and low

frequency sweeps

Z2real = [Z3real; Z4real $]$;

Z2imag = [Z3imag; Z4imag];

omega2 = [omega3; omega4];

Z3real = [Z5real; Z6real];

Z3imag = [Z5imag; Z6imag];

omega3 = $[$ omega5; omega6];

\% Strip off high frequency inductance data [i.e. data with +Imag(Z)] Omega1 $(1: 1: 8)=[] ; Z$ Z1real $(1: 1: 8)=[] ; \operatorname{Z1imag}(1: 1: 8)=[] ;$

omega2 $(1: 1: 19)=[] ; Z 2$ real $(1: 1: 19)=[] ; \operatorname{Z2imag}(1: 1: 19)=[] ;$

omega3 $(1: 1: 19)=[] ; Z 3 r e a l(1: 1: 19)=[] ; \operatorname{Z3imag}(1: 1: 19)=[] ;$

\% Subtract ohmic (electrolyte, wiring, etc) resistance from real(Z)

Z1_ohmic = Z1real $(1)$;

Z2_ohmic = Z2real $(1)$;

Z3_ohmic = Z3real $(1)$;

\% Convert impedance data to complex numbers and convert to area

\%specific resistance. Subtract out ohmic resistance from real

\%portion of $Z$. Divide by 2 since full cell measurements

Z1 = electrode_area*complex(Z1real-Z1_ohmic, Z1imag)./2;

Z2 = electrode_area ${ }^{*}$ complex(Z2real-Z2_ohmic, Z2imag)./2; 
Z3 = electrode_area ${ }^{*}$ complex(Z3real-Z3_ohmic, Z3imag)./2;

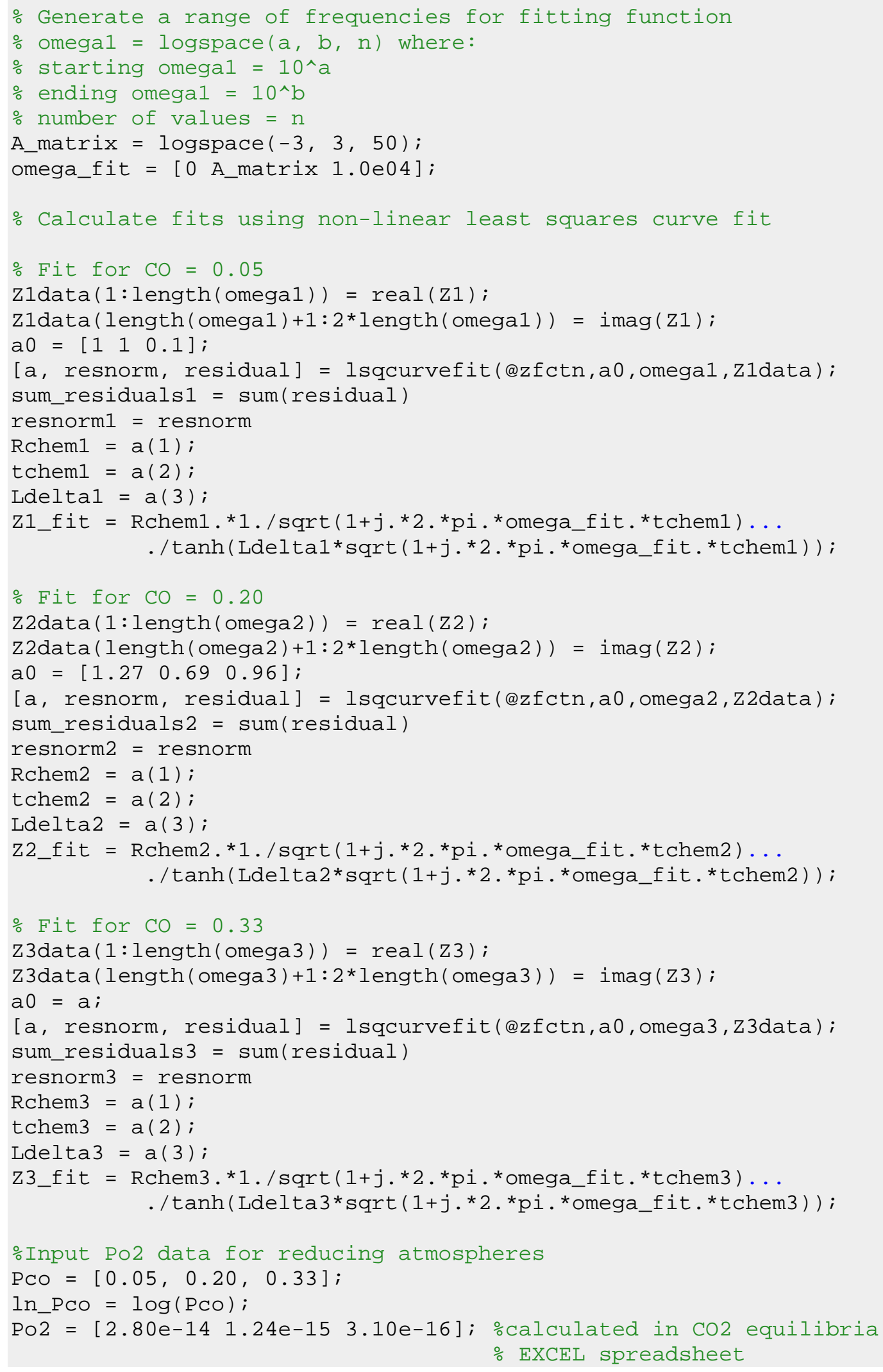


ln_Po2 $=\log (\mathrm{Po} 2)$;

\%Put Rchem, tchem, Ldelta data in vectors for plotting in Figure 2.

Rchemdata $=[$ Rchem1, Rchem2, Rchem3];

tchemdata $=[$ tchem1, tchem2, tchem3];

Ldelta $=[$ Ldelta1, Ldelta2, Ldelta3];

deloverLdata $=1 . /$ Ldelta;

ln_Rchemdata $=\log ($ Rchemdata $)$;

ln_tchemdata $=\log ($ tchemdata $)$;

ln_deloverLdata $=\log ($ deloverLdata $)$;

\%Fit In of parameters vs. In_Po2

$[\mathrm{p} 1, \mathrm{~S} 1]=\operatorname{polyfit}\left(\mathbf{l n} \_\right.$Pco, 1 n_Rchemdata, 1$)$;

$[\mathrm{p} 2, \mathrm{~S} 2]=$ polyfit $($ In_Pco, In_tchemdata, 1$)$;

$[\mathrm{p} 2 \mathrm{a}, \mathrm{S} 2 \mathrm{a}]=\operatorname{polyfit}\left(\mathbf{I} \_\right.$Po2, In_tchemdata, 1$)$;

$[\mathrm{p3}, \mathrm{s} 3]=\operatorname{polyfit}\left(\mathbf{l n} \_\right.$Pco, ln_deloverLdata, 1$)$;

\% Import A and delta calculated from 40 mol\% 0 non-stoich GDC data

$\%$ using idea defect model (model is in Zachau_thermo_factor_ver1.m)

$\%$ Set del0 $=0.20$, where del0 = mole fraction of $\mathrm{Gd}$ divided by 2;

$\operatorname{del} 0=0.20$;

deltaprime $=\left[\begin{array}{lll}0.0077 & 0.0097 & 0.0108\end{array}\right] ;$

delta $=$ del $\odot+$ deltaprime;

$A=\left[\begin{array}{lll}27.9139 & 22.6338 & 20.6156\end{array}\right]$;

$\mathrm{XV}=$ delta./2; \%vacancy molar fraction

cmc $=0.0827 ; \quad$ \%concentration of oxygen ion lattice sites

$\left(\mathrm{mol} / \mathrm{cm}^{\wedge} 3\right)$

$\mathrm{CV}=\mathrm{xv} \cdot{ }^{*} \mathrm{Cmc} ; \quad$ \%vacancy concentration

surf_area $=10000 ; \%$ surface area/unit volume $\left(\mathrm{cm}^{\wedge}-1\right)$ [estimate]

porosity $=0.5 ; \quad \%$ porosity of GDC electrode [estimate]

tortuosity $=1.5$; \%tortuosity of GDC electrode [estimate]

Rgas $=8.314 ; \quad$ \%ideal gas constant $\left(\mathrm{J} / \mathrm{mol}^{*} \mathrm{~K}\right)$

$\mathrm{F}=96478 ; \quad$ \%Faraday's constant (coulombs/equiv)

TinK $=900+273 ; \quad \%$ temperature (in K)

$\mathrm{L}=40 \mathrm{e}-4 ; \quad$ \%thickness of electrode layer $(\mathrm{cm})$

\% Calculate ro and Dv from Rchem and tchem data

ro $=c v \cdot{ }^{*}(1-$ porosity $) . /\left(A .{ }^{*}\right.$ surf_area.${ }^{*}$ tchemdata $)$

Dv = tortuosity./((1-porosity $) .{ }^{*} \mathrm{cv} .{ }^{*}$ surf_area.${ }^{*}$ ro $) \ldots$ . * (Rgas. *Tink./(2. ${ }^{*} \mathrm{~F}^{\wedge} 2 .{ }^{*}$ Rchemdata $\left.)\right) \cdot{ }^{\wedge} 2$

deloverL_calc $=\operatorname{sqrt}\left(A \cdot{ }^{*} D v \cdot{ }^{*}\right.$ tchemdata $) \cdot / \mathrm{L}$

phidata_calc $=1 . /$ deloverL_calc;

\% Calculate a thermodynamic factor from the fitted data

A_fit $=($ L./Ldelta $) . \wedge 2 . /$ Dv./tchemdata

\%Import ro data calculated from surface reaction rate constant data \%in Yashiro et al.(2002) page 474. 


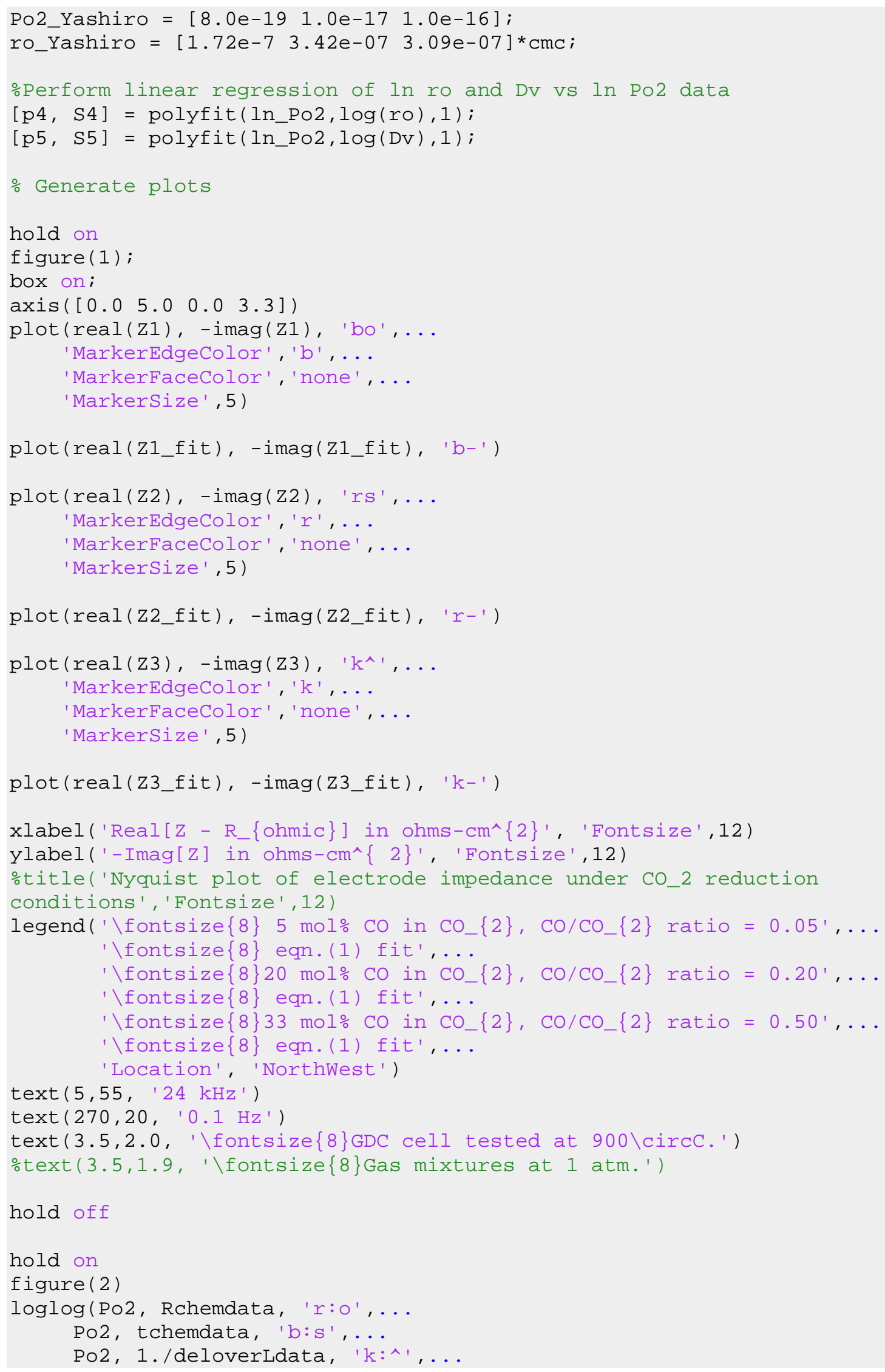


'MarkerSize' , 5)

hold on

plot(Po2, 1./deloverL_calc, 'k:^', ...

'MarkerFaceColor' ', 'k', . . .

'MarkerSize' , 5)

legend('\fontsize $\{8\} R_{-}\{$chem\}, fitted', ...

'\fontsize $\{8\} t$ t $\{$ chem $\}$, fitted', ...

'\fontsize\{8\}\phi, fitted',...

'\fontsize\{8\}\phi, calculated using eqn(4)', ...

'Location', 'Northwest' )

$\operatorname{axis}\left(\left[\begin{array}{llll}1.0 \mathrm{e}-16 & 1.0 \mathrm{e}-13 & 1 \mathrm{e}-01 & 100\end{array}\right)\right.$

\%title('Fit parameters for finite thickness electrode model', 'Fontsize', 12)

xlabel('\fontsize $\{10\} P_{-}\left\{0_{-}\{2\}\right\}$, in atm', 'Fontsize',12)

ylabel(' $\backslash$ fontsize $\{10\} R_{-}\{$chem $\}\left(\backslash\right.$ omega $\left.-\mathrm{cm}^{\wedge}\{2\}\right), t_{-}\{\mathrm{chem}\}(\mathrm{s}), \backslash$ phi (dimensionless)', 'Fontsize', 12)

text(0.2e-14,8.0, '\fontsize\{8\}GDC cell tested at 900\circC.') \%text $(0.2 \mathrm{e}-15,0.016$, '\fontsize $\{8\}$ Gas mixtures at $1 \mathrm{~atm} . ')$

hold off

hold on

figure(3)

$\log \log \left(\mathrm{Po} 2, \mathrm{~A}, \mathrm{~K} \mathrm{k}: \mathrm{o}^{\prime}, \ldots\right.$

Po2, A_fit, 'k:s', ...

'MarkerSize' , 5)

legend('\fontsize\{8\}Thermo factor, A, calc',...

'\fontsize\{8\}Thermo factor, A, fitted',...

'Location', 'SouthEast ')

axis([1.0e-16 1.0e-13 1e-01 1000])

\%title('Fit parameters for finite thickness electrode

model', 'Fontsize', 12)

xlabel('Ln P_\{0_\{2\}\}, in atm', 'Fontsize',12)

ylabel('Thermodynamic factor, dimensionless', 'Fontsize',12)

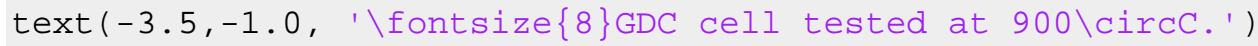

text $(-3.5,-0.8, ' \backslash$ fontsize $\{8\}$ Gas mixtures at 1 atm.' $)$

hold off

hold on

figure (4)

semilogx(omega1, real(Z1), 'ro',...

omega_fit, real(Z1_fit), ' $r:{ }^{\prime}, \ldots$

omega1, imag(Z1), 'bo', ...

omega_fit, imag(Z1_fit), 'b: ', . .

'MarkerSize', 5)

legend('\fontsize $\{8\}$ Real( $Z$ data) values',...

'\fontsize $\{8\}$ Real $z$ fit', ...

'\fontsize $\{8\} \operatorname{Imag}(Z$ data) values', ...

'\fontsize $\{8\}$ Imag $Z$ fit', ...

'Location', 'NorthEast')

\%title('Fit of Real Z \& Imag Z', 'Fontsize', 12)

xlabel('Frequency, in $\mathrm{Hz}^{\prime}$, 'Fontsize', 12)

ylabel('Real(Z), Imag(Z)', 'Fontsize',12)

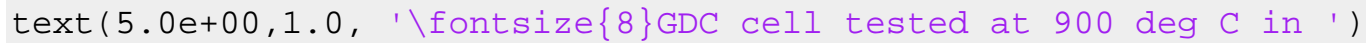




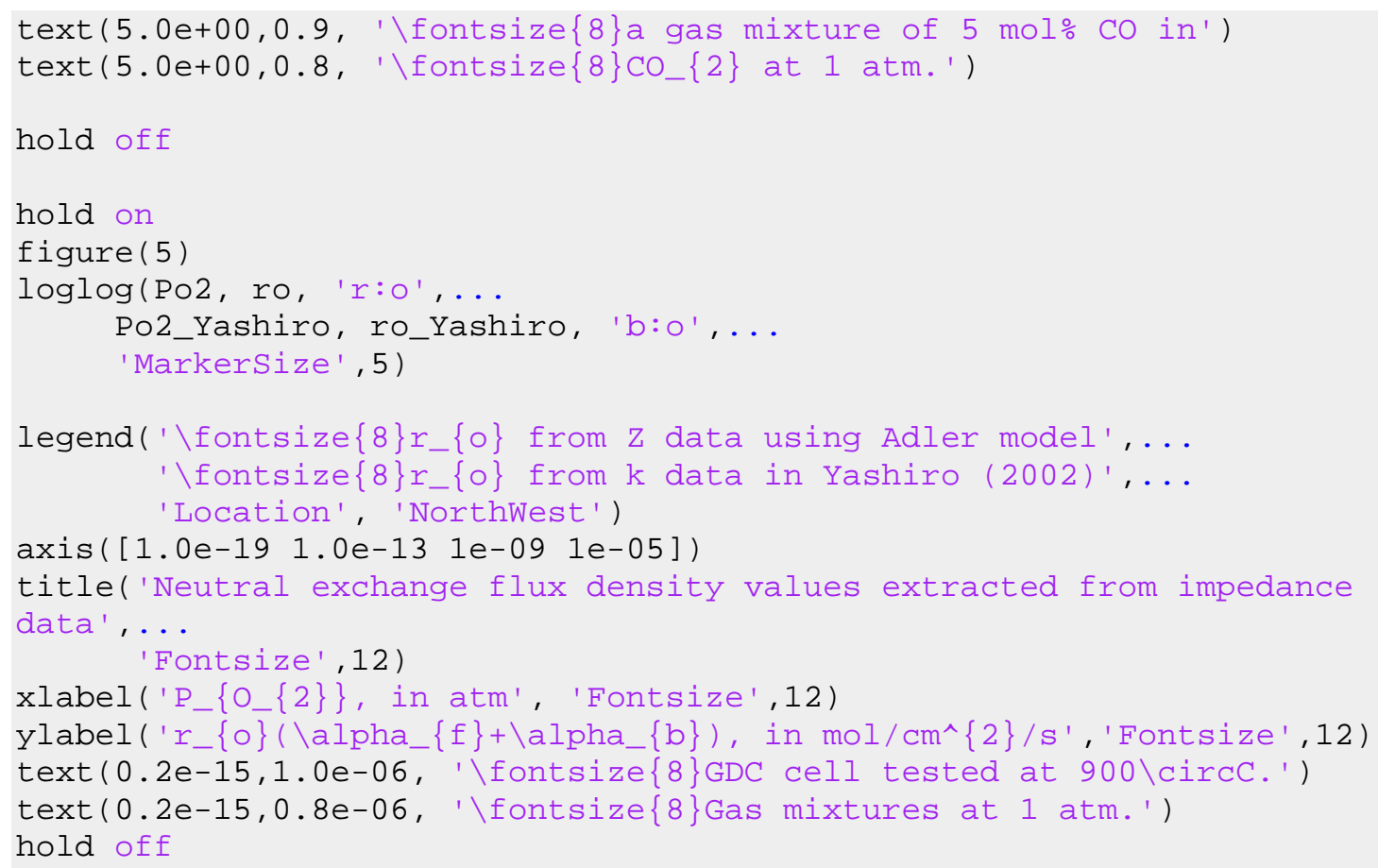

\%This function calculates the fit values for non-linear equation for Z:

$\% \quad Z=$ Rchem/ 
\%Concatenate real and imaginary values into a single output vector for

\%least squares minimization

Z_fit_out $(1:$ length $($ omega $))=$ Fitted_Real;

Z_fit_out (length(omega) $+1: 2^{*}$ length $($ omega $\left.)\right)=$ Fitted_Imag ;

end

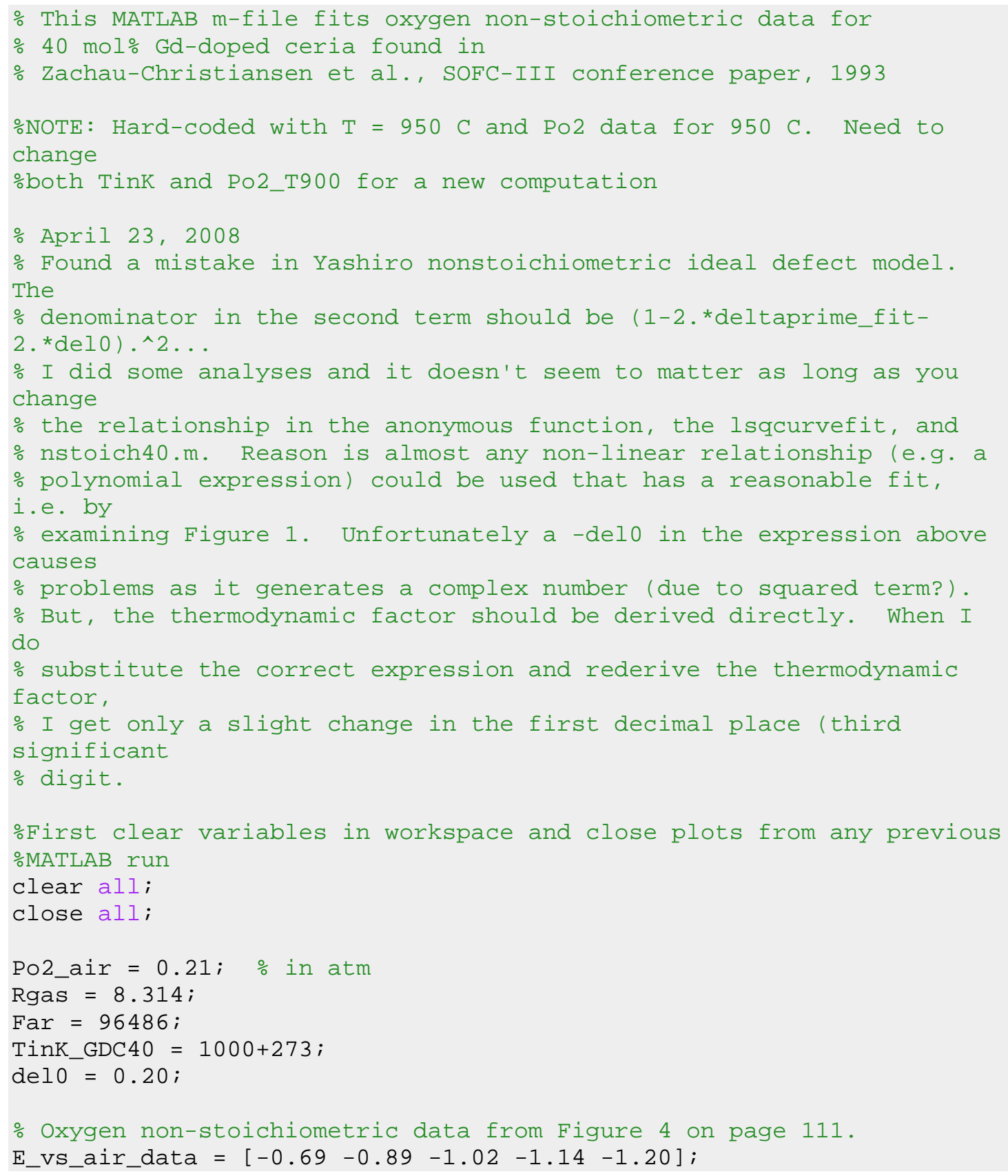


In_Po2_GDC40 = log (Po2_air $)+$

4. ${ }^{\star} \mathrm{Far} . / \mathrm{Rgas} . / \mathrm{TinK} \_\mathrm{GDC} 40 .{ }^{*} \mathrm{E} \_\mathrm{Vs} \_$air_data;

log_Po2_GDC40 $=\log 10(\exp ($ In_Po2_GDC40 $))$;

$\%$ 0x_data $=x$ where $\mathrm{Gd}(\mathrm{y}) \mathrm{Ce}(1-\mathrm{y}) 0(2-\mathrm{y} / 2+\mathrm{x})$

0x_data $=\left[\begin{array}{lllll}-0.01 & -0.02 & -0.03 & -0.04 & -0.05\end{array}\right]$;

deltaprime_GDC40 = -0x_data;

$a \odot=[1.00051 .0] ;$ \%Initial guess for fitting parameters

$\%$ Anonymous function for Yashiro's ideal point defect theory model

In_Po2_out $=@(a$, deltaprime_GDC40 $)-2 .{ }^{*} a(1) . /$ Rgas ./TinK_GDC40 ...

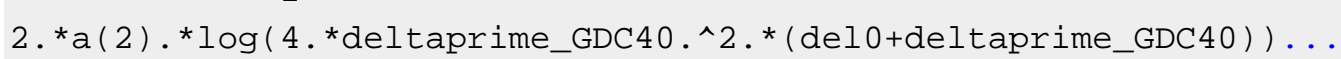
.$/\left(1-2 .{ }^{*}\right.$ deltaprime_GDC40+del0).^2./(2-deltaprime_GDC40-

del0), ...

'a', 'deltaprime_GDC40' ;

\% Now, call least squares curve fit function to fit non-stoich data

from

$\%$ Zachau

[a, resnorm, residual] $=$

lsqcurvefit(1n_Po2_out, a๑, deltaprime_GDC40,1n_Po2_GDC40) ;

sum_residuals1 = sum(residual)

resnorm1 = resnorm

delG_ideal $=\mathrm{a}(1)$;

deltaprime_fit $=0.001: 0.005: 0.10$;

In_Po2_fit $=-2 .{ }^{*}$ delG_ideal./Rgas./TinK_GDC40 ...

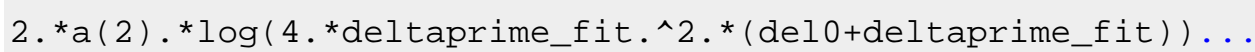

del0); .$/\left(1-2{ }^{*}\right.$ deltaprime_fit+del@).^2./(2-deltaprime_fit-

log_Po2_fit $=\log 10(\exp ($ ln_Po2_fit $))$;

\% Using fit, find nonstoich deltaprime for GDC electrode testing conditions

Po2_T900 $=[2.99 \mathrm{e}-131.33 \mathrm{e}-143.31 \mathrm{e}-15] ;$ \%this is data for $950 \mathrm{C}$

TinK $=950+273 ;$

Po2_scalar $=$ Po2_T900(1);

deltaprime $(1)=$ fzero(@(delprime) nstoich40(delprime, a, Po2_scalar, Tink, del0), 0.01 )

Po2_scalar $=$ Po2_T900 $(2)$;

deltaprime(2) = fzero(@(delprime) nstoich40(delprime, a, Po2_scalar, Tink, del0), 0.01 )

Po2_scalar $=$ Po2_T900(3);

deltaprime(3) = fzero(@(delprime) nstoich40(delprime, a, Po2_scalar, Tink, del0), $\odot .01)$

$\%$ Using fit, calculate the thermodynamic factor

$A=$ del0./deltaprime $-1 . /($ del0+deltaprime-2) $-(1+$ de 10$) \ldots$ .$/\left(\operatorname{del} 10+2 .{ }^{*}\right.$ deltaprime-1)

hold on

figure(1)

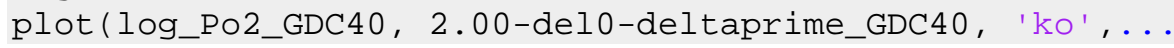


log_Po2_fit, 2.00-del0-deltaprime_fit, 'k:', ...

'MarkerSize', 5)

$\operatorname{axis}\left(\left[\begin{array}{llll}-21 & -10 & 1.74 & 1.80\end{array}\right]\right)$

box on

xlabel('Log P_\{0_\{2\}\}, in atm', 'Fontsize', 12)

ylabel('2 - Idelta, dimensionless', 'Fontsize',12)

legend(' Gd_\{0.4\}Ce_\{0.6\}0_\{2-\delta\} data', ...

' Fit of ideal point defect theory model', ...

hold off

'Location', 'NorthWest')

Published with MATLAB® 7.1 


\section{Bibliography}

[1] RJ Aaberg, R Tunold, S Tjelle, R Odegard, Oxidation of $\mathrm{CO}$ and $\mathrm{H}_{2}$ on $\mathrm{Ni} / \mathrm{YSZ}$ cermet electrodes, in: Poulsen FW, Bonanos N, Linderoth S, Mogensen M, ZachauChristiansen B (Eds.), High Temperature Electrochemistry: Ceramics and Metals, Proceedings of the 17th Riso International Symposium of Materials Science, Roskilde, Denmark ed., Riso National Laboratory, Roskilde, Denmark, 1996, pp. 511-516.

[2] SB Adler. Factors governing oxygen reduction in solid oxide fuel cell cathodes, Chem. Rev. 104 (2004) 4791-4843.

[3] SB Adler. Reference electrode placement in thin solid electrolytes, J. Electrochem. Soc. 149 (2002) E166-E172.

[4] SB Adler. Chemical expansivity of electrochemical ceramics, J Am Ceram Soc. 84 (2001) 2117-2119.

[5] SB Adler. Limitations of charge-transfer models for mixed-conducting oxygen electrodes, Solid State Ionics. 135 (2000) 603-612.

[6] SB Adler. Mechanism and kinetics of oxygen reduction on porous $\mathrm{La}_{1-\mathrm{x}} \mathrm{Sr}_{\mathrm{x}} \mathrm{CoO}_{3 \text {-delta }}$ electrodes, Solid State Ionics. 111 (1998) 125-134.

[7] SB Adler, XY Chen, JR Wilson. Mechanisms and rate laws for oxygen exchange on mixed-conducting oxide surfaces, J. Catalysis. 245 (2007) 91-109.

[8] SB Adler, BT Henderson, MA Wilson, DM Taylor, RE Richards. Reference electrode placement and seals in electrochemical oxygen generators, Solid State Ionics. 134 (2000) 35-42.

[9] SB Adler, JA Lane, BCH Steele. Fundamental Issues in Modeling of MixedConductors, A rebuttal to comments on "Electrode Kinetics of Porous Mixed-Conducting Oxygen Electrodes", J.Electrochem.Soc. 144 (1997) 1884-1890.

[10] SB Adler, JA Lane, BCH Steele. Electrode kinetics of porous mixed-conducting oxygen electrodes, J.Electrochem.Soc. 143 (1996) 3554-3564.

[11] E Aneggi, J Llorca, M Boaro, A Trovarelli. Surface-structure sensitivity of CO oxidation over polycrystalline ceria powders, J. Catal. 234 (2005) 88-95. 
[12] D Applebaum. Levy processes - from probability to finance and quantum groups, Notices of the AMS. 51 (2004) 1336-1347.

[13] RL Ash, WL Dowler, G Varsi. Feasibility of Rocket Propellant on Mars, Acta Astronautica. 5 (1978) 705-724.

[14] PW Atkins, Physical Chemistry, first ed., W. H. Freeman and Company, San Francisco, CA, 1978.

[15] A Atkinson, S Barnett, RJ Gorte, Irvine, J. T. S., AJ Mcevoy, M Mogensen, et al. Advanced anodes for high-temperature fuel cells, Nature Materials. 3 (2004) 17-27.

[16] JM Bae, Steele, B. C. H. Properties of $\mathrm{La}_{0.6} \mathrm{Sr}_{0.4} \mathrm{Co}_{0.2} \mathrm{Fe}_{0.8} \mathrm{O}_{3 \text {-delta }}$ (LSCF) double layer cathodes on gadolinium-doped cerium oxide (CGO) electrolytes - I. Role of $\mathrm{SiO}_{2}$, Solid State Ionics. 106 (1998) 247-253.

[17] AJ Bard, LR Faulkner, Electrochemical Methods: Fundamentals and Applications, second ed., Wiley, New York, NY, 2001.

[18] CR Barrett, WD Nix, AS Tetelman, The Principles of Engineering Materials, first ed., Prentice Hall, Englewood Cliffs, NJ, 1973.

[19] DM Bernardi, MW Verbrugge. Mathematical model of a gas diffusion electrode bonded to a polymer electrolyte, AICHE J. 37 (1991) 1151-1163.

[20] F Bidrawn, G Kim, G Corre, JTS Irvine, JM Vohs, RJ Gorte. Efficient Reduction of $\mathrm{CO}_{2}$ in a Solid Oxide Electrolyzer, Electrochem. Solid-State Lett. 11 (2008) B167-B170.

[21] JO Bockris, Reddy, A. K. N., M Gamboa-Aldeco, Modern Electrochemistry, Volume 2A, Fundamentals of Electrodics, second ed., Kluwer Academic/Plenum Publishers, New York, 2000.

[22] JO Bockris, Reddy, A. K. N., M Gamboa-Aldeco, Modern Electrochemistry 2A: Fundamentals of Electrodics, Second ed., Kluwer Academic/Plenum Publishers, New York, 2000.

[23] RJ Borg, GJ Dienes, An Introduction to Solid State Diffusion, first ed., Academic Press, San Diego, CA, 1988.

[24] M Boudart, Kinetics of Chemical Processes, Prentice-Hall, Englewood Cliffs, NJ, 1968. 
[25] BA Boukamp. Interpretation of an 'inductive loop' in the impedance of an oxygen ion conducting electrolyte/metal electrode system, Solid State Ionics. 143 (2001) 47-55.

[26] BA Boukamp, Bouwmeester, H. J. M. Interpretation of the Gerischer impedance in solid state ionics, Solid State Ionics. 157 (2003) 29-33.

[27] BA Boukamp, M Verbraeken, DHA Blanck, P Holtappels. SOFC-anodes, proof for a finite-length type Gerischer impedance? Solid State Ionics. 177 (2006) 2539-2541.

[28] FZ Boulenouar, K Yashiro, M Oishi, A Kaimai, Y Nigara, T Kawada, et al., Electrochemical oxidation of $\mathrm{CO}$ in a $\mathrm{CO}-\mathrm{CO}_{2}$ system a the interface of $\mathrm{Ni}$ grid electrode/YSZ electrolyte, in: Yokokawa H, Singhal SC (Eds.), Proceedings of the Seventh International Symposium on Solid Oxide Fuel Cells, SOFC VII, PV2001-16, Pennington, NJ ed., The Electrochemical Society, Pennington, NJ, 2001, pp. 759-768.

[29] Bouwmeester, H. J. M., AJ Burggraaf, Dense membranes for oxygen separation, in: Gellings PJ, Bouwmeester, H. J. M. (Eds.), The CRC Handbook of Solid State Electrochemistry, CRC Press, New York, 1997, pp. 503.

[30] BK Breedlove, GM Ferrence, J Washington, CP Kubiak. A photoelectrochemical approach to splitting carbon dioxide for a manned mission to Mars, Materials and Design. 22 (2001) 577-584.

[31] M Breysse, M Guenin, B Claudel, H Latreille, J Veron. Catalysis of carbon monoxide by cerium dioxide I. Correlations between catalytic activity and electrical conductivity, J. Catalysis. 27 (1972) 275-280.

[32] M Breysse, M Guenin, B Claudel, J Veron. Catalysis of carbon monoxide by cerium dioxide II. Microcalorimetric investigation of adsorption and catalysis, J. Catalysis. 28 (1973) 54-62.

[33] T Bunluesin, H Cordatos, RJ Gorte. A study of CO oxidation on Rh/ceria, J. Catalysis. 157 (1995) 222-226.

[34] T Bunluesin, H Cordatos, RJ Gorte. A study of CO oxidation kinetics on Rh/Ceria, J. Catalysis. 157 (1995) 222-226.

[35] VA Burrows, S Sundaresan, YJ Chabal. Real-time study of self-sustained oscillations on the CO oxidation rate on Pt, J. Vac. Sci. Technol. A. 5 (1987) 801-804. 
[36] SH Chan, XJ Chen, KA Khor. Cathode micromodel of solid oxide fuel cell, J. Electrochem. Soc. 151 (2004) A164-A172.

[37] HW Chandler, FZ Pollara. Oxygen Regeneration in a Solid Electrolyte System, AICHE Chemical Engineering Progress Series: Aerospace Life Support. 62 (1966) 38-42.

[38] XY Chen, JS Yu, SB Adler. Thermal and chemical expansion of Sr-doped lanthanum cobalt oxide (La-1-xSrxCoO3-delta), Chemistry of Materials. 17 (2005) 45374546.

[39] K Choy, W Bai, S Clarojrochkul, BCH Steele. The development of intermediatetemperature solid oxide fuel cells for the next millennium, J.Power Sources. 71 (1998) 361-369.

[40] GW Coffey, LR Pederson, DM Ponds, PC Rieke, EC Thomsen. Fabrication and characterization of a novel Luggin capillary reference electrode for use in solid state electrolytes, Solid State Ionics. 178 (2007) 827-832.

[41] COMSOL AB, COMSOL Multiphysics ${ }^{\circledR}$ Users’ Guide, version 3.2 (2005).

[42] COMSOL AB, COMSOL Multiphysics ${ }^{\circledR}$ Modeling Guide, version 3.2 (2005).

[43] A Constantinides, N Mostoufi, Numerical Methods for Chemical Engineers with MATLAB Applications, first ed., Prentice Hall PTR, Upper Saddle River, NJ, 1999.

[44] P Costamagna, P Costa, V Antonucci. Micro-modeling of solid oxide fuel cell electrodes, Electrochim. Acta. 43 (1998) 375-394.

[45] J Crank, The Mathematics of Diffusion, second ed., Oxford University Press, New York, NY, 1975.

[46] SC Crow, The MOXCE Project: New Cells for Producing Oxygen on Mars, $33^{\text {rd }}$ AIAA/ASME/SAE/ASEE Joint Propulsion Conference and Exhibit, AAIA 97-2766, (1997).

[47] LS Darken, RW Gurry. The system iron-oxygen. I. The Wustite field and related equilibria, J. Amer. Chem Soc. 67 (1945) 1398-1411.

[48] JW Dawicke, RN Blumenthal. Oxygen association pressure measurements on nonstoichiometric cerium dioxide, J. Electrochem. Soc. 133 (1986) 904-909. 
[49] De Groot, S. R., P Mazur, Non-Equilibrium Thermodynamics, first ed., NorthHolland, Amsterdam, the Netherlands, 1962.

[50] S Dikmen, P Shuk, M Greenblatt, H Gocmez. Hydrothermal synthesis and properties of $\mathrm{Ce}_{1-\mathrm{x}} \mathrm{Gd}_{\mathrm{x}} \mathrm{O}_{2 \text {-delta }}$ solid solutions, Solid State Sciences. 4 (2002) 585-590.

[51] M Doyle, JP Meyers, J Newman. Computer simulations of the impedance response of lithium rechargeable batteries, J. Electrochem. Soc. 147 (2000) 99-110.

[52] BG Drake, D Cooke, Reference Mission 3.0, Addendum to the Human Exploration of Mars: The Reference Mission of the NASA Mars Exploration Study Team, NASA internal publication EX13-98-036, (1998) 12-13.

[53] LA Dunyushkina, SB Adler. Influence of electrolyte surface planarization on the performance of the porous SOFC cathodes, J. Electrochem. Soc. 152 (2005) A2040A2045.

[54] LA Dunyushkina, YX Lu, SB Adler. Microelectrode array for isolation of electrode polarization on planar solid electrolytes, J. Electrochem. Soc. 152 (2005) A1668-A1676.

[55] K Eguchi, T Setoguchi, T Inoue, H Arai. Electrical properties of ceria-based oxides and their application to solid oxide fuel cells, Solid State Ionics. 52 (1992) 165-172.

[56] L Elikan, DH Archer, RL Zahradnik. Oxygen Regeneration in Solid Electrolyte Batteries: Fundamental Considerations, AICHE Chemical Engineering Progress Series: Aerospace Life Support. 62 (1966) 29-37.

[57] L Elikan, JP Morris, Solid oxide electrolyte system for oxygen regeneration, NASA CR-1359 (1969) 1-166.

[58] L Elikan, JP Morris, CK Wu, Development of a solid oxide electrolyte carbon dioxide and water reduction system for oxygen recovery, NASA CR-2014 (1972) 1-169.

[59] TH Etsell, SN Flengas. Electrical properties of solid oxide electrolytes, Chem. Rev. 70 (1970) 339-376.

[60] TH Etsell, SN Flengas. Overpotential behavior of stabilized zirconia solid electrolyte fuel cells, J. Electrochem. Soc. 118 (1971) 1890-1900.

[61] J Faber, C Goeffroy, A Roux, A Sylvestre, P Abelard. A systematic investigation of the DC electrical conductivity of rare-earth doped ceria, Appl. Phys. A. 49 (1989) 225. 
[62] G Fafilek. The use of voltage probes in impedance spectroscopy, Solid State Ionics. 176 (2005) 2023-2029.

[63] HJ Freund, MW Roberts. Surface chemistry of carbon dioxide, Surface Science Reports. 25 (1996) 225-273.

[64] TF Fuller, Ph.D. dissertation, Solid-Polymer-Electrolyte Fuel Cells, University of California, Berkeley, CA (1992).

[65] C Gabrielli, Identification of electrochemical processes by frequency response analysis, 004/83 (1980) 3-6.

[66] H Gerischer, Z. Phys. Chem. 198 (1951) 216.

[67] S Gewies, WG Bessler. Physically based impedance modeling of Ni/YSZ cermet anodes, J. Electrochem. Soc. 155 (2008) B937-B952.

[68] J Giner, C Hunter. The mechanism of operation of the Teflon-Bonded gas diffusion electrode: a mathematical model, J.Electrochem.Soc. 116 (1969) 1124-1130.

[69] M Gong, X Liu, J Trembly, C Johnson. Sulfur-tolerant anode materials for solid oxide fuel cell application, J. Power Sources. 168 (2007) 289-298.

[70] RJ Gorte, S Park, JM Vohs, C Wang. Anodes for direct oxidation of dry hydrocarbons in a solid-oxide fuel cell, Adv. Mater. 12 (2000) 1465-1469.

[71] RD Green, CC Liu, SB Adler. Carbon dioxide reduction on gadolinia-doped ceria cathodes, Solid State Ionics. 179 (2008) 647-660.

[72] V Grover, AK Tyagi. Phase relations in the $\mathrm{CeO}_{2}-\mathrm{Gd}_{2} \mathrm{O}_{3}-\mathrm{ZrO}_{2}$ system, J. Solid State Chem. 177 (2004) 4197-4204.

[73] Q Guo, RE White. A steady-state impedance model for a PEMFC cathode, J. Electrochem. Soc. 151 (2004) E133-E149.

[74] J Ha, SM Martin, Y Jeon, IJ Yoon, RB Brown, H Nam, et al. A polymeric junction membrane for solid-state reference electrodes, Anal.Chim.Acta. 549 (2005) 59-66.

[75] D Halliday, R Resnick, J Walker, Fundamentals of Physics Extended, fifth ed., Wiley, New York, NY, 1997. 
[76] A Hashibon, S Raz, I Riess. Preferred position for the reference electrode in solid state electrochemistry, Solid State Ionics. 149 (2002) 167-176.

[77] A Hauch, SH Jensen, JB Bilde-Sorensen, M Mogensen. Silica segregation in the Ni/YSZ electrode, J. Electrochem. Soc. 154 (2007) A619-A626.

[78] U Hennings, R Reimert. Investigation of the structure and the redox behavior of gadolinium doped ceria to select a suitable composition as catalyst support in the steam reforming of natural gas, Appl. Catal. A-Gen. 325 (2007) 41-49.

[79] AF Hepp, GA Landis, CP Kubiak, A Chemical Approach to Carbon Dioxide Utilization on Mars, (1993) 799-817.

[80] JS Herring, P Lessing, JE O'Brien, C Stoots, J Hartvigsen, S Elangovan, Hydrogen production through high-temperature electrolysis in a solid oxide cell, 2004.

[81] SJ Hoffman, DI Kaplan, Human Exploration of Mars: The Reference Mission of the NASA Mars Exploration Study Team, NASA Special Publication 6107, (1997) 3-101-3105.

[82] SJ Hoffman, DI Kaplan. Human Exploration of Mars: The Reference Mission of the NASA Mars Exploration Study Team, NASA Special Publication. 6107 (1997) 98-036.

[83] DK Hohnke. Ionic conduction in doped oxides with the fluorite structure, Solid State Ionics. 5 (1981) 531-534.

[84] P Holtappels, J Bradley, Irvine, J. T. S., A Kaiser, M Mogensen. Electrochemical characterization of ceramic SOFC anodes, J. Electrochem. Soc. 148 (2001) A923-A929.

[85] P Holtappels, De Haart, L. G. J., U Stimming, IC Vinke, M Mogensen. Reaction of CO/CO2 gas mixtures on Ni-YSZ cermet electrodes, J.Appl.Electrochem. 29 (1999) 561568.

[86] RV Homsy, J Newman. An asymptotic solution for the Warburg impedance of a rotating disk electrode, J. Electrochem. Soc. 121 (1974) 521-523.

[87] SJ Hong, AV Virkar. Lattice parameters and densities of rare-earth oxide doped ceria electrolytes, J. Am. Ceram. Soc. 78 (1995) 433-439. 
[88] T Horita, K Yamaji, N Sakai, M Ishikawa, H Yokokawa, T Kawada, et al. Oxygen surface exchange of $\mathrm{Y}_{0.2} \mathrm{Ce}_{0.8} \mathrm{O}_{2-\mathrm{x}}$ under reducing atmosphere, Electrochem. Solid-State Lett. 1 (1998) 4-6.

[89] G Hsieh, SJ Ford, TO Mason, LR Pederson. Experimental limitations in impedance spectroscopy: Part VI - four point measurements of solid state materials systems, Solid State Ionics. 100 (1997) 297-311.

[90] G Hsieh, SJ Ford, TO Mason, LR Pederson. Experimental limitations in impedance spectroscopy: Part I - simulation of reference electrode artifacts in three-point measurements, Solid State Ionics. 91 (1996) 191-201.

[91] G Hsieh, TO Mason, EJ Garboczi, LR Pederson. Experimental limitations in impedance spectroscopy: Part III - effect of reference electrode geometry/position, Solid State Ionics. 96 (1997) 153-172.

[92] G Hsieh, TO Mason, LR Pederson. Experimental limitations in impedance spectroscopy: Part II - electrode artifacts in three-point measurements on Pt/YSZ, Solid State Ionics. 91 (1996) 203-212.

[93] JS Huebner. Oxygen fugacity values of furnace gas mixtures, Am. Mineral. 60 (1975) 815-823.

[94] RP Iczkowski, MB Cutlip. Voltage losses in fuel cell cathodes, J. Electrochem. Soc. 127 (1980) 1433-1440.

[95] H Idriss, MA Barteau, Active Sites on Oxides: From Single Crystals to Catalysts, in: Gates BC, Knozinger H (Eds.), Impact of Surface Science on Catalysis, Academic Press, San Diego, CA, 2000, pp. 262-448.

[96] RP Ingel, D Lewis. Lattice-parameters and density for $\mathrm{Y}_{2} \mathrm{O}_{3}$-stabilized $\mathrm{ZrO}_{2}$, J. Am. Ceram. Soc. 69 (1986) 325-332.

[97] International Centre for Diffraction Data (ICDD), Powder Diffraction File. PDF-4 (2007).

[98] SH Jensen, Ph.D. dissertation, Solid oxide electrolyser cell, Risø-PhD-29(EN), Risø Labs, Roskilde, Denmark (2006). 
[99] SH Jensen, Hogh, J. V. T., R Barfod, M Mogensen, High temperature electrolysis of steam and carbon dioxide, in PL Sonderberg, H Larsen (Eds), Proceedings of The Risø International Energy Conference, Roskilde, Denmark (2003) 204-215.

[100] SH Jensen, PH Larsen, M Mogensen. Hydrogen and synthetic fuel production from renewable energy sources, Int J Hydrogen Energy. 32 (2007) 3253-3257.

[101] KV Jensen, R Wallenberg, I Chorkendorff, M Mogensen. Effect of impurities on structural and electrochemical properties of the Ni-YSZ interface, Solid State Ionics. 160 (2003) 27-37.

[102] Y Jiang, AV Virkar. Fuel composition and diluent effect of gas transport and performance of anode-supported SOFCs, J. Electrochem. Soc. 150 (2003) A942-A951.

[103] T Jin, T Okuhara, GJ Mains, JM White. Temperature-programmed desorption of $\mathrm{CO}$ and $\mathrm{CO} 2$ from $\mathrm{Pt} / \mathrm{CeO} 2$. An important role for lattice oxygen in $\mathrm{CO}$ oxidation, $\mathrm{J}$. Phys. Chem. 91 (1987) 3310-3315.

[104] MJ Jorgensen, S Primdahl, M Mogensen. Characterization of composite SOFC cathodes using electrochemical impedance spectroscopy, Electrochim. Acta. 44 (1999) 4195-4201.

[105] K Kammer, M Mogensen. Oxidation of methane and hydrogen on Ce1-xGdxO2delta fluorites, Electrochem. Solid-State Lett. 8 (2005) A108-A109.

[106] SV Karpachev, AE Zupniik, SF Pal'guev. Polarization of carbon monoxide electrodes on platinum in a solid zirconia lime electrolyte, Electrochim. Acta. 9 (1964) 1681-1685.

[107] T Kato, A Momma, Y Kaga, S Nagata, Y Kasuga, M Kitase. Influence of cell configuration on measuring interfacial impedances between a solid electrolyte and an electrode, Solid State Ionics. 132 (2000) 287-295.

[108] M Katsuki, S Wang, K Yasumoto, M Dokiya. The oxygen transport in Gd-doped ceria, Solid State Ionics. 154-155 (2002) 589-595.

[109] TJ Kelly, Moon Lander: How we developed the Apollo Lunar Module, first ed., Smithsonian Institution Press, Washington, 2001. 
[110] MA Khaleel, JR Selman, Cell, Stack, and System Modeling, in: Singhal SC, Kendall K (Eds.), High Temperature Solis Oxide Fuel Cells: Fundamentals, Design, and Applications, first ed., Elsevier Ltd., Kidlington, Oxford, UK, 2003, pp. 405-322.

[111] JA Kilner. Fast oxygen transport in acceptor doped oxides, Solid State Ionics. 129 (2000) 13-23.

[112] H Kim, S Park, J Vohs, RJ Gorte. Direct oxidation of liquid fuels in a solid oxide fuel cell, J. Electrochem. Soc. 148 (2001) A693-A695.

[113] H Kim, C Lu, WL Worrell, JM Vohs, RJ Gorte. Cu-Ni cermet anodes for direct oxidation of methane in solid-oxide fuel cells, J.Electrochem.Soc. 149 (2002) A247A250.

[114] C Kittel, Introduction to Solid State Physics, sixth ed., Wiley, New York, NY, 1986.

[115] P Knauth, HL Tuller. Solid-State Ionics: roots, status and future prospects, J. Am. Ceram. Soc. 85 (2002) 1654-1680.

[116] T Kobayashi, S Wang, M Dokiya, H Tagawa, T Hashimoto. Oxygen nonstoichiometry of $\mathrm{Ce}_{1-\mathrm{y}} \mathrm{Sm}_{\mathrm{y}} \mathrm{O}_{2-0.5 y-\mathrm{x}}$, Solid State Ionics. 126 (1999) 349-357.

[117] FA Kroger, HJ Vink, Relations between the concentrations of imperfections in crystalline solids, in: Seitz F, Turnull D (Eds.), Solid State Physics - Advances in Research and Applications, Academic Press, New York, NY, 1956, pp. 431-435.

[118] T Kudo, H Obayashi. Oxygen ion conduction of the fluorite-type $\mathrm{Ce}_{1-\mathrm{x}} \mathrm{Ln}_{\mathrm{x}} \mathrm{O}_{2-\mathrm{x} / 2}(\mathrm{Ln}$ = Lanthanoid element), J. Electrochem. Soc. 122 (1975) 142-147.

[119] K Lackner, Global Research Technologies LLC, Global Research Technologies web site: http://www.grestech.com/ (last accessed 12/1/2008).

[120] W Lai, SM Haile. Impedance spectroscopy as a tool for chemical and electrochemical analysis of mixed conductors: A case study of ceria, J. Am. Ceram. Soc. 88 (2005) 2979-2997.

[121] JA Lane, JA Kilner. Oxygen surface exchange on gadolinia doped ceria, Solid State Ionics. 136-137 (2000) 927-932. 
[122] Lankhorst, M. H. R., Bouwmeester, H. J. M., H Verweij. Use of the rigid band formalism to interpret the relationship between $\mathrm{O}$ chemical potential and electron concentration in $\mathrm{La}_{1-\mathrm{x}} \mathrm{Sr}_{\mathrm{x}} \mathrm{CoO}_{3 \text {-delta, }}$ Phys. Rev. Lett. 77 (1996) 2989-2992.

[123] GO Lauvstad, R Tunold, S Sunde. Electrochemical oxidation of CO on Pt and Ni point electrodes in contact with an yttria-stabilized zirconia electrolyte - I. Modeling of steady-state and impedance behavior, J.Electrochem.Soc. 149 (2002) E497-E505.

[124] GO Lauvstad, R Tunold, S Sunde. Electrochemical oxidation of CO on Pt and Ni point electrodes in contact with an yttria-stabilized zirconia electrolyte - II. Steady-state and impedance measurements, J.Electrochem.Soc. 149 (2002) E506-E514.

[125] G Lauvstad, R Tunold, S Sunde, Oxidation of carbon monoxide on strontium ferrate, 95-1 (1995) 731-740.

[126] JS Lewis, MS Matthews, ML Guerrieri, Resources of Near-Earth Space, in: Gehrels T (Ed.), Space Science Series, first ed., The University of Arizona Press, Tucson and London, 1993.

[127] YL Liu, S Primdahl, M Mogensen. Effects of impurities on microstructure in Ni/YSZ-YSZ half-cells for SOFC, Solid State Ionics. 161 (2003) 1-10.

[128] Z Liu, JS Wainright, W Huang, RF Savinell. Positioning the reference electrode in proton exchange membrane fuel cells: calculations of primary and secondary current distribution, Electrochim. Acta. 49 (2004) 923-935.

[129] JR Macdonald, WB Johnson, Fundamentals of Impedance Spectroscopy, in: Macdonald JR (Ed.), Impedance Spectroscopy, first ed., Wiley, New York, NY, 1987, pp. $1-26$.

[130] J Maier, Physical Chemistry of Ionic Materials: Ions and Electrons in Solids, John Wiley \& Sons Ltd., West Sussex, England, 2004.

[131] J Maier. On the correlation of macroscopic and microscopic rate constants in solid state chemistry, Solid State Ionics. 112 (1998) 197-228.

[132] PS Manning, JD Sirman, JA Kilner. Oxygen self-diffusion and surface exchange studies of oxide electrolytes having the fluorite structure, Solid State Ionics. 93 (1996) 125-132. 
[133] OA Marina, C Bagger, S Primdahl, M Mogensen. A solid oxide fuel cell with a gadolinia-doped ceria anode: preparation and performance, Solid State Ionics. 123 (1999) 199-208.

[134] OA Marina, M Mogensen. High temperature conversion of methane on a composite gadolinia-doped ceria-gold electrode, Appl. Catal. A. 189 (1999) 117-126.

[135] Y Matsuzaki, I Yasuda. Electrochemical oxidation of $\mathrm{H}_{2}$ and $\mathrm{CO}$ in a $\mathrm{H}_{2}-\mathrm{H}_{2} \mathrm{O}-\mathrm{CO}-$ $\mathrm{CO}_{2}$ system at the interface of a Ni-YSZ cermet electrode and YSZ electrolyte, J. Electrochem. Soc. 147 (2000) 1630-1635.

[136] Y Matsuzaki, I Yasuda. The poisoning effect of sulfur-containing impurity gas on an SOFC anode: Part I. Dependence on temperature, time, and impurity concentration, Solid State Ionics. 132 (2000) 261-269.

[137] S McIntosh, SB Adler, JM Vohs, RJ Gorte. Effect of polarization on and implications for characterization of LSM-YSZ composite cathodes, Electrochemical and Solid State Letters. 7 (2004) A111-A114.

[138] S McIntosh, RJ Gorte. Direct hydrocarbon solid oxide fuel cells, Chem. Rev. 104 (2004) 4845-4865.

[139] S McIntosh, JM Vohs, RJ Gorte. Role of hydrocarbon deposits in the enhanced performance of direct-oxidation SOFCs, J.Electrochem.Soc. 150 (2003) A470-A476.

[140] S McIntosh, JM Vohs, RJ Gorte. An examination of lanthanide additives on the performance of Cu-YSZ cermet anodes, Electrochim. Acta. 47 (2002) 3815-3821.

[141] CP McKay, TR Meyer, PJ Boston, M Nelson, T Maccallum, O Gwynne, Utilizing Martian resources for life support, (1993) 819-843.

[142] IS Metcalfe. Carbon Monoxide Oxidation over Platinum Supported on Yttriastabilized Zirconia, Brit. Ceram. Proc. 43 (1988) 139-150.

[143] IS Metcalfe, S Sundaresan. Oxygen Transfer between Metals and Oxygen-Ion Conducting Supports, AICHE Journal. 34 (1988) 195-208.

[144] GA Mills. Status and future opportunities for conversion of synthesis gas to liquid fuels, Fuel. 73 (1994) 1243-1279. 
[145] J Mizusaki, H Tagawa, Y Miyaki, S Yamauchi, K Fueki, I Koshiro, et al. Kinetics of the Electrode-Reaction at the $\mathrm{CO}-\mathrm{CO}_{2}$, Porous Pt/stabilized Zirconia Interface, Solid State Ionics. 53-56 (1992) 126-134.

[146] G Mogensen, M Mogensen. Reduction Reactions in Doped Ceria Ceramics Studied by Dilatometry, Thermochimica Acta. 214 (1993) 47-50.

[147] M Mogensen, Ceria-based electrodes, in: Trovarelli A (Ed.), Catalysis by Ceria and Related Materials, vol. 2, Imperial College Press, London, 2002, pp. 453-481.

[148] M Mogensen, T Lindegaard, UR Hansen, G Mogensen. Physical-Properties of Mixed Conductor Solid Oxide Fuel-Cell Anodes of Doped $\mathrm{CeO}_{2}$, J. Electrochem. Soc. 141 (1994) 2122-2128.

[149] M Mogensen, D Lybye, K Kammer, N Bonanos, Ceria revisited: electrolyte or electrode material? 2005-07 (2005) 1069-1074.

[150] M Mogensen, NM Sammes, GA Tompsett. Physical, chemical, and electrochemical properties of pure and doped ceria, Solid State Ionics. 129 (2000) 63-94.

[151] M Mogensen, KV Jensen, MJ Jorgensen, S Primdahl. Progress in understanding SOFC electrodes, Solid State Ionics. 150 (2002) 123-129.

[152] M Mogensen, KV Jensen, MJ Jorgensen, S Primdahl. Progress in understanding SOFC electrodes, Solid State Ionics. 150 (2002) 123-129.

[153] M Mogensen, S Primdahl, MJ Jorgensen, C Bagger. Composite Electrodes in Solid Oxide Fuel Cells and Similar Solid State Devices, Journal of Electroceramics. 5 (2000) 141-152.

[154] T Mori, J Drennan, Y Wang, JH Lee, Jg Li, T Ikegami. Electrolytic Properties and Nanostructural Features in the $\mathrm{La}_{2} \mathrm{O}_{3}-\mathrm{CeO}_{2}$ System, J. Electrochem. Soc. 150 (2003) A665-A673.

[155] EP Murray, T Tsai, SA Barnett. A direct-methane fuel cell with a ceria-based anode, Nature. 400 (1999) 649-651.

[156] M Nagata, Y Itoh, H Iwahara. Dependence of observed voltages on the positioning of the reference electrode on the solid electrolyte, Solid State Ionics. 67 (1994) 215-224. 
[157] T Nakamura, T Kobayashi, K Yashiro, A Kaimai, T Otake, K Sato, et al.

Electrochemical behaviors of mixed conducting oxide anodes for solid oxide fuel cell, J. Electrochem. Soc. 6 (2008) B563-B569.

[158] T Nakamura, T Kobayashi, K Yashiro, K Sato, T Kawada, J Mizusaki, Electrochemical behaviors of mixed conducting anodes for SOFC, ECS Transactions. 7 (2007) 1601-1607.

[159] National Aeronautics and Space Administration (NASA), The Vision for Space Exploration, NP-2004-01-334-HQ, NASA, Washington, D.C., 2004.

[160] J Newman. Resistance of flow of current to a disk, J. Electrochem. Soc. 113 (1966) 501-502.

[161] JS Newman, Electrochemical Systems, second ed., Prentice-Hall, Englewood Cliffs, NJ, 1991.

[162] JS Newman, CW Tobias. Theoretical analysis of current distribution in porous electrodes, J.Electrochem.Soc. 109 (1962) 1183-1191.

[163] J Newman, KE Thomas-Alyea, Electrochemical Systems, third ed., WileyInterscience, Hoboken, NJ, 2004.

[164] J Newman, W Tiedemann. Porous electrode theory with battery applications, AICHE J. 21 (1975) 25-41.

[165] RAH Niessen, PHL Notten. Reference electrode-induced surface poisoning of thinfilm electrodes, J.Electrochem.Soc. 152 (2005) A2051-A2057.

[166] JE O'Brien, CM Stoots, JS Herring, PA Lessing, JJ Hartvigsen, S Elangovan. Performance measurements of solid-oxide electrolysis cells for hydrogen production, J.Fuel Cell Sci.Technol. 2 (2005) 156-163.

[167] R Odegard, J Keller, GA Landis, Oxygen generation and storage for a Mars sample return mission utilizing in-situ resources, AAIA 2007-6066, (2007).

[168] Ostergard, M. J. L., C Clausen, C Bagger, M Mogensen. Manganite-zirconia composite cathodes for SOFC: influence of structure and composition, Electrochim. Acta. 40 (1995) 1971-1981. 
[169] JY Park, ED Wachsman. Lower temperature electrolytic reduction of $\mathrm{CO}_{2}$ to $\mathrm{O}_{2}$ and $\mathrm{CO}$ with high-conductivity solid oxide bilayer electrolytes, J. Electrochem. Soc. 8 (2005) A1654-A1659.

[170] J Park, RN Blumenthal. Electronic Transport in 8 mole percent $\mathrm{Y}_{2} \mathrm{O}_{3}-\mathrm{ZrO}_{2}$, J. Electrochem. Soc. 136 (1989) 2867-2876.

[171] S Park, JM Vohs, RJ Gorte. Direct oxidation of hydrocarbons in a solid-oxide fuel cell, Nature. 404 (2000) 265-267.

[172] D Perez-Coll, D Marrero-Lopez, JC Ruiz-Morales, P Nunez, Abrantes, J. C. C., JR Frade. Reducibility of $\mathrm{Ce}_{1-\mathrm{x}} \mathrm{Gd}_{\mathrm{x}} \mathrm{O}_{2 \text {-delta }}$ in prospective working conditions, J. Power Sources. 173 (2007) 291-297.

[173] D Perez-Coll, P Nunez, JC Ruiz-Morales, J Pena-Martinez, JR Frade. Reexamination of bulk and grain boundary conductivities of $\mathrm{Ce}_{1-\mathrm{x}} \mathrm{Gd}_{\mathrm{x}} \mathrm{O}_{2 \text {-delta }}$ ceramics, Electrochim. Acta. 52 (2007) 2001.

[174] ML Perry, J Newman, EJ Cairns. Mass transport in gas-diffusion electrodes: a diagnostic tool for fuel-cell cathodes, J. Electrochem. Soc. 145 (1998) 5-15.

[175] G Prentice, Electrochemical Engineering Principles, first ed., Prentice-Hall, Englewood Cliffs, NJ, 1991.

[176] S Primdahl, M Mogensen, Gas conversion impedance: SOFC anodes in $\mathrm{H}_{2}-\mathrm{H}_{2} \mathrm{O}$ atmospheres, in: Stimming U, Singhal SC, Tagawa H, Lahnert W (Eds.), Proceedings of the Fifth International Symposium on Solid Oxide Fuel Cells, SOFC V, PV97-40, Pennington, NJ ed., The Electrochemical Society, Pennington, NJ, 1997, pp. 530-537.

[177] S Primdahl, M Mogensen. Durability and thermal cycling of Ni/YSZ cermet anodes for solid oxide fuel cells, J.Appl.Electrochem. 30 (2000) 247-257.

[178] S Primdahl, M Mogensen. Gas diffusion impedance in characterization of solid oxide fuel cell anodes, J.Electrochem.Soc. 146 (1999) 2827-2833.

[179] S Primdahl, M Mogensen. Gas conversion impedance: A test geometry effect in characterization of solid oxide fuel cell anodes, J.Electrochem.Soc. 145 (1998) 24312438. 
[180] M Primet, E Garbowski, Fundamentals and applications of ceria in combustion reactions, in: Trovarelli A (Ed.), Catalysis by Ceria and Related Materials, vol. 2, Imperial College Press, London, 2002, pp. 407-429.

[181] MN Rahaman, Ceramic Processing, first ed., CRC Press, Boca Raton, FL, 2007.

[182] D Rapp, A review of Mars ISPP technology, JPL D-15223 (1998) 60-64.

[183] LF Razon, RA Schmitz. Intrinsically Unstable Behavior during the Oxidation of Carbon-Monoxide on Platinum, Catalysis Reviews-Science and Engineering. 28 (1986) 89-164.

[184] DM Reed, HU Anderson, W Huebner. Characterization of solid oxide fuel cells by use of an internal Pt voltage probe, J. Electrochem. Soc. 143 (1996) 1558-3759.

[185] R Richter, Basic Investigation into the Production of Oxygen in a Solid Electrolyte, AIAA-81-1175, (1981) 1-14.

[186] I Riess, Electrochemistry of Mixed Ionic-Electronic Conductors, Chapter 7, The CRC Handbook of Solid State Electrochemistry, first edition ed., CRC Press, Boca Raton, FL, 1997, pp. 223-268.

[187] HM Rosenburg, The Solid State, third ed., Oxford Univervsity Press, Oxford, England, 1988.

[188] E Ruiz-Trejo, J Maier. Electronic transport in single crystals of Gd-doped ceria, J. Electrochem. Soc. 154 (2007) B583-B587.

[189] J Rutman, I Riess. Reference electrodes for thin-film solid-state ionic devices, Solid State Ionics. 179 (2008) 108-112.

[190] J Rutman, I Riess. Placement of reference electrode in solid electrolyte cells, Solid State Ionics. 179 (2008) 913-918.

[191] J Rutman, I Riess. Placement of reference electrode in solid electrolyte cells, Electrochim. Acta. 52 (2007) 6073-6083.

[192] BC Sales, JE Turner, MB Maple. Oscillatory oxidation of CO over Pt, Pd, and Ir catalysts: Theory, Surface Science. 114 (1982) 381-394. 
[193] CM Sanchez-Sanchez, V Montiel, DA Tryk, A Aldaz, A Fujishima.

Electrochemical approaches to alleviation of the problem of carbon dioxide accumulation, Pure Appl. Chem. 73 (2001) 1917-1927.

[194] T Setoguchi, K Okamoto, K Eguchi, H Arai. Effects of Anode Material and Fuel on Anodic Reaction of Solid Oxide Fuel-Cells, J.Electrochem.Soc. 139 (1992) 2875-2880.

[195] RD Shannon. Revised effective radii and systematic studies of interatomic distances in halides and chalcogenides, Acta Crystallogr. Sec. A. 32 (1976) 751-767.

[196] Z Shao, SM Haile. A high-performance cathode for the next generation of solidoxide fuel cells, Nature. 431 (2004) 170-173.

[197] M Shelef, GW Graham, RW McCabe, Ceria and other oxygen storage components in automotive catalysts, in: Trovarelli A (Ed.), Catalysis by Ceria and Related Materials, vol. 2, Imperial College Press, London, 2002, pp. 343-365.

[198] RJ Sheppard, BP Jordan, EH Grant. Least squares analysis of complex data with applications to permittivity measurements, J. Phys. D: Appl. Phys. 3 (1970) 1759-1764.

[199] BS Singh, KR Sridhar, Research and Technology Needs for Chemical Processes and Operations on Mars, in: RG Galloway, S Lokaj (Eds.), Proceedings of the Sixth Annual Exposition on Engineering Construction and Operations in Space, American Society of Civil Engineers, Reston, VA (1998) 245-254.

[200] RH Socolow. Can we bury global warming? Sci. Am. 293 (2005) 49-55.

[201] JJ Sprague, O Porat, HL Tuller. Mixed conducting gas sensors: atmosphere dependent electrode impedance, Sensors and Actuators B. 35 (1996) 348-352.

[202] TE Springer, TA Zawodzinski, S Gottesfeld. Polymer electrolyte fuel cell model, J. Electrochem. Soc. 138 (1991) 2334-2342.

[203] KR Sridhar, R Foerstner. Regenerative solid oxide fuel cells for Mars exploration, J. Propul. Power. 16 (2000) 1105-1111.

[204] KR Sridhar, R Foerstner, Regenerative $\mathrm{CO} / \mathrm{CO}_{2}$ Solid Oxide Fuel Cells for Mars Exploration, AIAA $36^{\text {th }}$ Aerospace Sciences Meeting and Exhibit, AIAA 98-0650 (1998) $1-8$. 
[205] KR Sridhar, BT Vaniman. Oxygen production on Mars using solid oxide electrolysis, Solid State Ionics. 93 (1997) 321-328.

[206] ML Stancati, JC Niehoff, WC Wells, RL Ash, Remote Automated Propellant Production: a New Potential for Round Trip Spacecraft, AIAA 79-0906 (1979) 262-270.

[207] Steele, B. C. H., JM Bae. Properties of $\mathrm{La}_{0.6} \mathrm{Sr}_{0.4} \mathrm{Co}_{0.2} \mathrm{Fe}_{0.8} \mathrm{O}_{3-\mathrm{x}}$ (LSCF) double layer cathodes on gadolinium-doped cerium oxide (CGO) electrolytes - II. Role of oxygen exchange and diffusion, Solid State Ionics. 106 (1998) 255-261.

[208] BCH Steele. Materials for IT-SOFC stacks: 35 years R\&D: the inevitability of gradualness? Solid State Ionics. 134 (2000) 3-20.

[209] BCH Steele, KM Hori, S Uchino. Kinetic parameters influencing the performance of IT-SOFC composite electrodes, Solid State Ionics. 135 (2000) 445-450.

[210] CM Stoots, JE O'Brain, GL Hawkes, JS Herring, JJ Hartvigsen, High temperature co-electrolysis of $\mathrm{H}_{2} \mathrm{O}$ and $\mathrm{CO}_{2}$ for syngas production, 30 (Nov. 13-17 2006) Abstract \#418.

[211] CM Stoots, JE O'Brien, JS Herring. Progress in high-temperature electrolysis for the production of hydrogen, Trans. Am. Nucl. Soc. 92 (2005) 112-113.

[212] AM Sukeshini, B Habibzadeh, BP Becker, CA Stoltz, BW Eichhorn, GS Jackson. Electrochemical oxidation of $\mathrm{H}_{2}, \mathrm{CO}$, and $\mathrm{CO} / \mathrm{H}_{2}$ mixtures on patterned $\mathrm{Ni}$ anodes on YSZ electrolytes, J.Electrochem.Soc. 153 (2006) A705-A715.

[213] S Sunde. Simulations of Composite Electrodes in Fuel Cells, J. Electroceram. 5 (2000) 153-182.

[214] AM Svensson, S Sunde, K Nisanhcioglu. Mathematical modeling of oxygen exchange and transport in air-perovskite-YSZ interface regions I. Reduction of intermediately adsorbed oxygen, J. Electrochem. Soc. 144 (1997) 2719-2732.

[215] AM Svensson, S Sunde, K Nisanhcioglu. Mathematical modeling of oxygen exchange and transport in air-perovskite-YSZ interface regions II. Direct exchange of oxygen vacancies, J. Electrochem. Soc. 145 (1998) 1390-1400.

[216] T Takeguchi, Y Kani, T Yano, R Kikuchi, K Eguchi, K Tsujimoto, et al. Study on steam reforming of $\mathrm{CH}_{4}$ and $\mathrm{C}_{2}$ hydrocarbons and carbon deposition on Ni-YSZ cermets, J. Power Sources. 112 (2002) 588. 
[217] CW Tanner, KZ Fung, AV Virkar. Oxide fuel cell performance I. Theoretical analysis, J. Electrochem. Soc. 144 (1997) 21-30.

[218] G Tao, KR Sridhar, CL Chan. Study of carbon dioxide electrolysis at electrode/electrolyte interface: Part I. Pt/YSZ interface, Solid State Ionics. 175 (2004) 615-619.

[219] G Tao, KR Sridhar, CL Chan. Study of carbon dioxide electrolysis at electrode/electrolyte interface: Part II. Pt-YSZ cermet/YSZ interface, Solid State Ionics. 175 (2004) 621-624.

[220] S Tao, Irvine, J. T. S. Investigation of the mixed conducting oxide ScYZT as a potential SOFC material, J. Electrochem. Soc. 151 (2004) A497-A503.

[221] DP Thunnissen, D Rapp, CJ Voorhees, SF Dawson, CS Guernsey, A 2007 Mars Sample Return Mission utilizing in-situ propellant production, AIAA 37th Aerospace Sciences Meeting and Exhibit, AIAA-1999-851 (1999) 1-20.

[222] Z Tianshu, P Hing, H Huang, J Kilner. Ionic conductivity in the $\mathrm{CeO}_{2}-\mathrm{Gd}_{2} \mathrm{O}_{3}$ system $(0.05<\mathrm{Gd} / \mathrm{Ce}<0.4)$ prepared by oxalate coprecipitation, Solid State Ionics. 148 (2002) 567-573.

[223] A Trovarelli, Structural properties and non-stoichiometric behavior of $\mathrm{CeO}_{2}$, in: Trovarelli A (Ed.), Catalysis by Ceria and Related Materials, vol. 2, Imperial College Press, London, 2002, pp. 15-50.

[224] EE Underwood, Quantitative Stereology, Addison-Wesley, Reading, MA, 1970.

[225] MM Viitanen, RG v. Welzenis, HH Brongersma, FPF van Berkel. Silica poisoning of oxygen membranes, Solid State Ionics. 150 (2002) 223-228.

[226] DY Wang, AS Nowick. Cathodic and Anodic Polarization Phenomena at PlatinumElectrodes with Doped Ceo2 as Electrolyte .1. Steady-State Overpotential, J.Electrochem.Soc. 126 (1979) 1155-1165.

[227] DY Wang, AS Nowick. Cathodic and Anodic Polarization Phenomena at PlatinumElectrodes with Doped Ceo2 as Electrolyte .2. Transient Overpotential and Ac Impedance, J.Electrochem.Soc. 126 (1979) 1166-1172.

[228] S Wang, $\mathrm{H}$ Inaba, $\mathrm{H}$ Tagawa. Nonstoichiometry of $\mathrm{Ce}_{0.8} \mathrm{Gd}_{0.2} \mathrm{O}_{1.9-\mathrm{x}}, \mathrm{J}$. Electrochem. Soc. 144 (1997) 4076-4080. 
[229] S Wang, H Inaba, H Tagawa, M Dokiya, T Hashimoto. Nonstoichiometry of $\mathrm{Ce}_{0.9} \mathrm{Gd}_{0.1} \mathrm{O}_{1.95-\mathrm{x}}$, Solid State Ionics. 107 (1998) 73-79.

[230] S Wang, T Kobayashi, M Dokiya, T Hashimoto. Electrical and ionic conductivity of Gd-doped ceria, J. Electrochem. Soc. 147 (2000) 3606-3609.

[231] S Wang, van der Heide, P.A.W., C Chavez, AJ Jacobson, SB Adler. An electrical conductivity relaxation study of $\mathrm{La}_{0.6} \mathrm{Sr}_{0.4} \mathrm{Fe}_{0.8} \mathrm{CO}_{0.2} \mathrm{O}_{3 \text {-delta, }}$, Solid State Ionics. 156 (2003) 201-208.

[232] W Weppner, Electrode performance, in: Bruce PG (Ed.), Solid State

Electrochemistry, first ed., Cambridge University Press, Cambridge, UK, 1995, pp. 204.

[233] AC West, J Newman. Interpretation of kinetic rate data taken in a channel flow cell, J. Electrochem. Soc. 136 (1989) 3755-3759.

[234] MF Wilkes, P Hayden, AK Bhattacharya. Catalytic studies on ceria lanthana solid solutions II. Oxidation of carbon monoxide, J. Catalysis. 219 (2003) 295-304.

[235] JR Wilson, W Worawarit, R Mendoza, HY Chen, JM Hiller, DJ Miller, K Thornton, PW Voorhees, SB Adler, SA Barnett. Three-dimensional reconstruction of a solid-oxide fuel-cell anode, Nature Materials. 5 (2006) 541-544.

[236] JR Wilson, SB Adler, DT Schwartz. Full-spectrum nonlinear response of a sinusoidally modulated rotating disk electrode, Phys. Fluids. 17 (2005) 063601.

[237] JR Wilson, DT Schwartz, SB Adler. Nonlinear electrochemical impedance spectroscopy for solid oxide fuel cell cathode materials, Electrochim. Acta. 51 (2006) 1389-1402.

[238] J Winkler, PV Hendriksen, N Bonanos, M Mogensen. Geometric requirements of solid electrolyte cells with a reference electrode, J.Electrochem.Soc. 145 (1998) 11841192.

[239] K Yashiro, S Onuma, A Kaimai, Y Nigara, T Kawada, J Mizusaki, et al. Mass transport properties of $\mathrm{Ce}_{0.9} \mathrm{Gd}_{0.1} \mathrm{O}_{2 \text {-delta }}$ at the surface and in the bulk, Solid State Ionics. 152-153 (2002) 469-476.

[240] I Yasuda, T Hikita. Precise determination of the chemical diffusion coefficient of calcium-doped lanthanum chromites by means of electrical conductivity relaxation, J. Electrochem. Soc. 141 (1994) 1268-1273. 
[241] B Zachau-Christianson, T Jacobsen, S Skaarup. Electrochemical determination of oxygen stoichiometry and entropy in oxides, Solid State Ionics. 86-88 (1996) 725-731.

[242] B Zachau-Christianson, T Jacobsen, K West, S Skaarup, Electrochemical determination of the oxygen stoichiometry of doped ceria, in: Singhal SC, Iwahara $\mathrm{H}$ (Eds.), Proceedings of the Third International Symposium on Solid Oxide Fuel Cells, SOFC III, PV93-4, Pennington, NJ ed., The Electrochemical Society, Pennington, NJ, 1993, pp. 104-111.

[243] RL Zahradnik. Diffusional processes in solid electrolyte fuel cell electrodes, J. Electrochem. Soc. 117 (1970) 1443-1446.

[244] TS Zhang, J Ma, LB Kong, SH Chan, JA Kilner. Aging behavior and ionic conductivity of ceria-based ceramics: a comparative study, Solid State Ionics. 170 (2004) 209-217.

[245] R Zubrin, The Case for Mars, Free Press, New York, NY, 1996.

[246] R Zubrin, S Price, Mars Sample Return mission utilizing in-situ propellant production; Final Report, NASA-CR-188371 (1995) 1-69. 